\title{
Visible-Light-Promoted Oxo-Sulfonylation of Ynamides with Sulfonic Acids
}

Lu Wang, ${ }^{\dagger}$ Chengrong Lu, ${ }^{\ddagger}$ Yanni Yue, ${ }^{* \dagger}$ Chao Feng ${ }^{*, \dagger}$

Institute of Advanced Synthesis (IAS), School of Chemistry and Molecular Engineering, Nanjing Tech University, 30 South Puzhu Road, Nanjing 211816, P. R. China

${ }^{\ddagger}$ College of Chemistry, Chemical Engineering and Materials Science, Soochow University, Su zhou 215123, P. R. China

\section{Table of Contents}

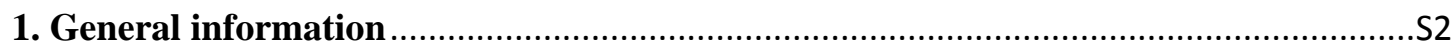

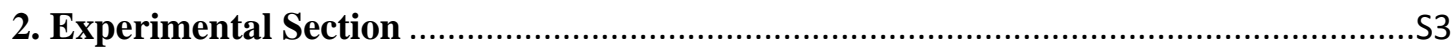

2.1 General procedures for the preparation of ynamides.................................................. 3

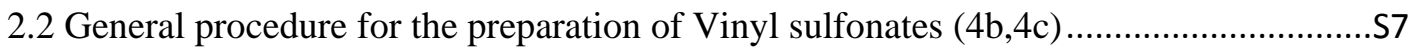

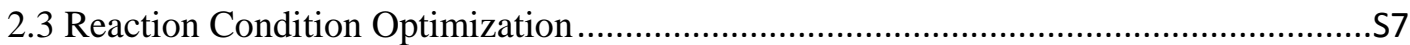

2.4 General Procedure for Oxo-sulfonylation of Ynamides with Sulfonic Acids ..............S10

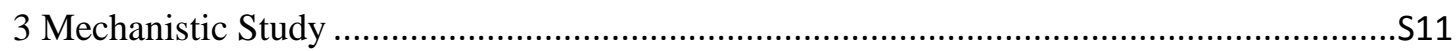

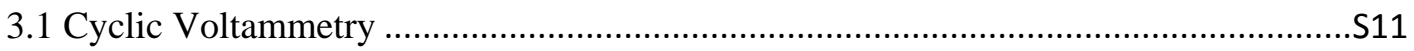

3.2 Emission quenching experiments (Stern- Volmer studies) .........................................S11

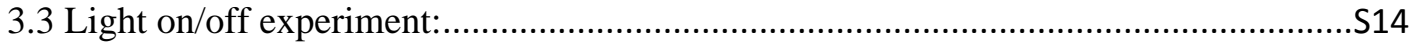

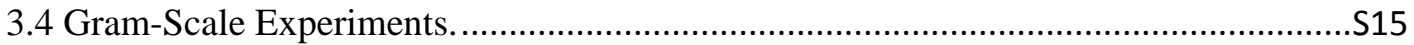

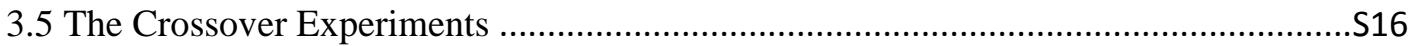

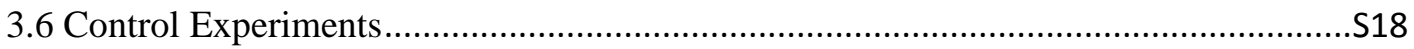

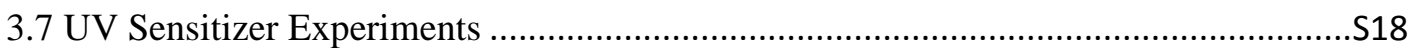

3.8 Determination of the reaction quantum yield $(\Phi)$ : ......................................................

3.9 DFT Calculation on the Triplet Energies of intermediate 4a: .................................S22

4. Characterization of structurally novel compounds ........................................................ 227

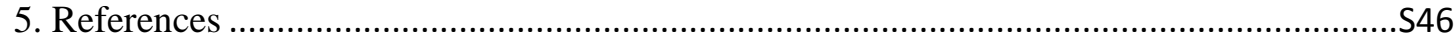

6. ${ }^{1} \mathrm{H}$ and ${ }^{13} \mathrm{C}$ NMR spectra of structurally novel compounds ............................................. 


\section{General information}

All solvent dried by passage through a column of neutral alumina under nitrogen prior to use. All other reagents were obtained from commercial sources and used without further purification unless otherwise noted. Flash chromatography was performed using Merck silica gel 60 with distilled solvents. Thin-layer chromatography (TLC) was performed on Silicycle $250 \mu \mathrm{m}$ silica gel plates visualized under UV light. HRMS spectra were recorded on a Waters Q-TOF Permier Spectrometer. ${ }^{1} \mathrm{H}$ NMR and ${ }^{13} \mathrm{C}$ NMR spectra were recorded using Bruker Avance $400 \mathrm{MHz}$ spectrometers. Chemical shifts for ${ }^{1} \mathrm{H}$ NMR spectra are reported as $\delta$ in units of parts per million (ppm) downfield from $\mathrm{SiMe}_{4}(\delta 0.0)$ and relative to the signal of chloroform- $d(\delta 7.26$, singlet). Multiplicities were given as: $\mathrm{s}$ (singlet); $\mathrm{d}$ (doublet); $\mathrm{t}$ (triplet); $\mathrm{q}$ (quartet); $\mathrm{dd}$ (doublet of doublets); dt (doublet of triplets); m (multiplet) and etc. Coupling constants are reported as a $J$ value in $\mathrm{Hz}$. Carbon nuclear magnetic resonance spectra $\left({ }^{13} \mathrm{C} \mathrm{NMR}\right)$ are reported as $\delta$ in units of parts per million (ppm) downfield from $\mathrm{SiMe}_{4}(\delta 0.0)$ and relative to the signal of chloroform-d ( $\delta 77.00$, triplet). The photocatalysts were prepared following literature procedures ${ }^{[11]}$. All the sulfonic acids were purchased from commercial suppliers and used as received unless otherwise noted. 


\section{Experimental Section}

\subsection{General procedures for the preparation of ynamides}

\section{Method A: Preparation of ynamides (1a-1o, 1r-1s, 1v-1x, 1aa-1ab) ${ }^{[1,2,4,6]}$}

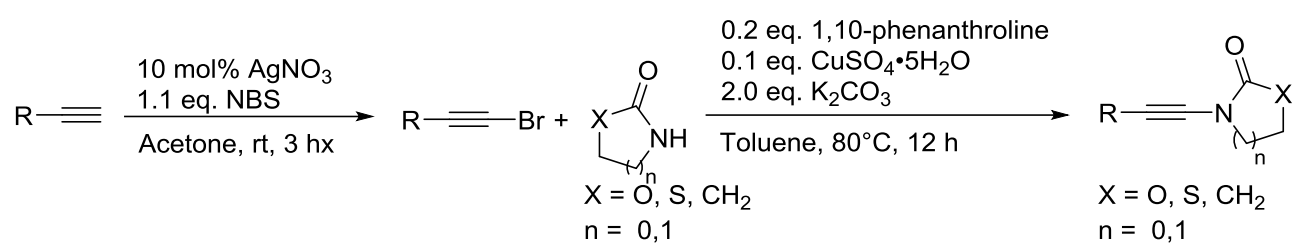

Synthetic procedure: a) To a solution of alkyne (1.0 equiv, $10 \mathrm{mmol})$ in acetone $(0.1$ $\mathrm{M}, 100 \mathrm{~mL})$ was added NBS (1.1 equiv, $11 \mathrm{mmol})$ and $\mathrm{AgNO}_{3}(10 \mathrm{~mol} \%, 1 \mathrm{mmol})$ the resulting mixture was stirred under nitrogen for $3 \mathrm{~h}$ at room temperature. After removing excess acetone, the reaction was quenched with water, and the organic layer was extracted with pentane $(30 \mathrm{~mL} \times 3)$, organic layer was dried over $\mathrm{Na}_{2} \mathrm{SO}_{4}$, the organic solvent was evaporated, and the residue was subjected to column chromatography on silica gel (petroleum ether) to obtain pure colorless oil of acetylene bromide.

b) To a dried flask was added oxazolidin-2-one (1.0 equiv, $5 \mathrm{mmol}), \mathrm{CuSO}_{4} \cdot 5 \mathrm{H}_{2} \mathrm{O}(10$ mol\%, $0.5 \mathrm{mmol}), 1,10$-phenanthroline (20 mol\%, $1 \mathrm{mmol}$ ) and $\mathrm{K}_{2} \mathrm{CO}_{3}$ (2.0 equiv, 10 mmol), the mixture was subsequently treated with anhydrous toluene $(0.1 \mathrm{M}, 50 \mathrm{~mL})$ and acetylene bromide (1.2 equiv, $6 \mathrm{mmol})$. The reaction mixture was caped under a blanket of nitrogen and heated in an oil bath at $70-80{ }^{\circ} \mathrm{C}$ for $12 \mathrm{~h}$. After complete conversion of starting material, the reaction mixture was cooled to room temperature, filtered through Celite, and concentrated in vacuo. The methanol was removed and the residue obtained was purified by column chromatography on silica gel (petroleum ether : ethyl acetate $=7: 3$ ) to afford the desired product.

Method B: Preparation of ynamides (1p-1q, 1t-1u, 1y) ${ }^{[7,8,10]}$
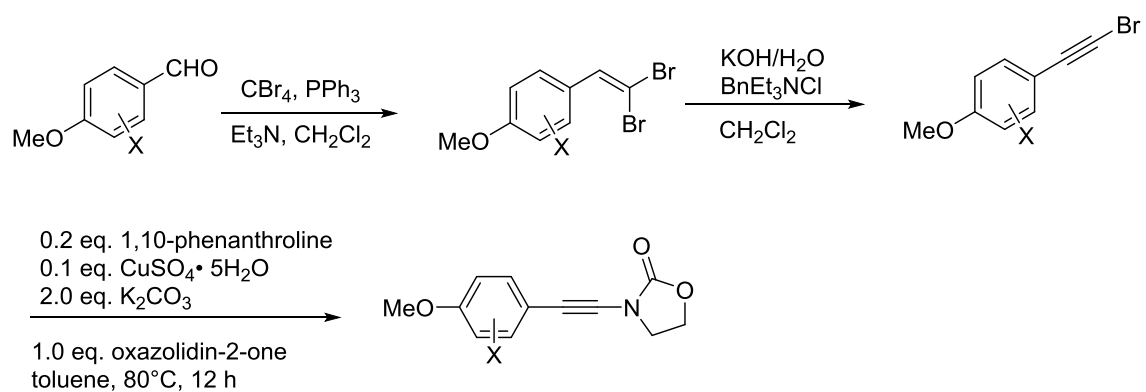
a) To a solution of $\mathrm{CBr}_{4}(11.1 \mathrm{~g}, 33.41 \mathrm{mmol})$ in $\mathrm{CH}_{2} \mathrm{Cl}_{2}(30 \mathrm{~mL})$ stirred at $0{ }^{\circ} \mathrm{C}$ was added triphenyl phosphine $(17.6 \mathrm{~g}, 67.0 \mathrm{mmol})$ portion-wise. The resulting solution was stirred at $0{ }^{\circ} \mathrm{C}$ for $30 \mathrm{~min}$. Aldehyde $(25.7 \mathrm{mmol})$ and triethylamine $(3.37 \mathrm{~mL}, 25.7$ mmol) were dissolved in $\mathrm{CH}_{2} \mathrm{Cl}_{2}(30 \mathrm{~mL})$ and added slowly to the ylide solution via a cannula over a period of $5 \mathrm{~min}$. The resulting mixture was stirred at $0{ }^{\circ} \mathrm{C}$ for $1 \mathrm{~h}$. Hexanes $(400 \mathrm{~mL})$ were added to the solution and stirred for $30 \mathrm{~min}$ at $0{ }^{\circ} \mathrm{C}$ and filtered cold through a bed of silica gel. The solution was concentrated and purified by silica gel chromatography (petroleum ether) to afford dibromoalkene.

b) To a solution of the dibromoalkene (2.0 equiv, $20.9 \mathrm{mmol}$ ) and benzyltriethylammonium chloride (1.0 equiv, $10.4 \mathrm{mmol})$ in $\mathrm{CH}_{2} \mathrm{Cl}_{2}(0.125 \mathrm{M}, 80 \mathrm{~mL})$ rapidly stirred at $0{ }^{\circ} \mathrm{C}$ was added a solution of $\mathrm{KOH}(54.0 \mathrm{~g})$ in $\mathrm{H}_{2} \mathrm{O}(40 \mathrm{~mL})$. The solution was stirred for $1 \mathrm{~h}$ at $0{ }^{\circ} \mathrm{C}$, and then was extracted with $\mathrm{CH}_{2} \mathrm{Cl}_{2}$. The organic extracts were dried over $\mathrm{Na}_{2} \mathrm{SO}_{4}$, filtered, and concentrated in vacuo. The product can be used without further purification, but if desired, it can be purified by silica gel flash column chromatography (EtOAc/hexane) to afford acetylenic bromides.

c) To a dried flask was added oxazolidin-2-one (1.0 equiv, $5 \mathrm{mmol}), \mathrm{CuSO}_{4} \cdot 5 \mathrm{H}_{2} \mathrm{O}(10$ mol\%, $0.5 \mathrm{mmol}), 1,10$-phenanthroline (20 mol\%, $1 \mathrm{mmol})$ and $\mathrm{K}_{2} \mathrm{CO}_{3}$ (2.0 equiv, 10 $\mathrm{mmol})$, the mixture was subsequently treated with anhydrous toluene $(0.1 \mathrm{M}, 50 \mathrm{~mL})$ and acetylene bromide (1.2 equiv, $6 \mathrm{mmol}$ ). The reaction mixture was caped under a blanket of nitrogen, and heated in an oil bath at $70-80{ }^{\circ} \mathrm{C}$ for $12 \mathrm{~h}$. After complete conversion of starting material, the reaction mixture was cooled to room temperature, filtered through Celite, and concentrated in vacuo. The methanol was removed and the residue obtained was purified by column chromatography on silica gel (petroleum ether : ethyl acetate $=13: 7$ ) to afford the desired product.

Method C: Preparation of ynamides (1ac-1ag) ${ }^{[3,5,6,7]}$

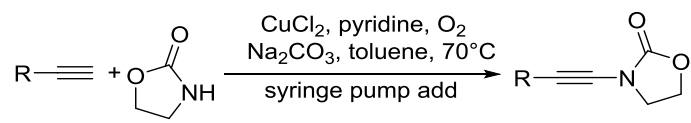

To a mixture of oxazolidinone (1.0 equiv, $5.0 \mathrm{mmol}$ ), $\mathrm{Na}_{2} \mathrm{CO}_{3}$ (2.0 equiv, $10.0 \mathrm{mmol}$ ), and $\mathrm{CuCl}_{2}(10 \mathrm{~mol} \%, 0.5 \mathrm{mmol})$ in a round bottom flask were added toluene $(0.1 \mathrm{M}$, 
$50 \mathrm{~mL}$ ) and pyridine (40 mol\% $2.0 \mathrm{mmol})$. The reaction was placed under a balloon of $\mathrm{O}_{2}$, stirred and heated to $70{ }^{\circ} \mathrm{C}$. Alkyne (1.0 equiv, $\left.5.0 \mathrm{mmol}\right)$ in toluene $(0.1 \mathrm{M}, 50 \mathrm{~mL})$ was then added via syringe pump addition over $5 \mathrm{~h}$, and the reaction stirred for another $8 \mathrm{~h}$. Upon completion, the reaction mixture was cooled to rt, filtered through Celite, and concentrated in vacuo. The crude residue was purified by silica gel flash column chromatography (petroleum ether: ethyl acetate $=4: 1$ ) to afford ynamide as a colourless oil.

Method D: Preparation of ynamides (1z-1ab) ${ }^{[9]}$

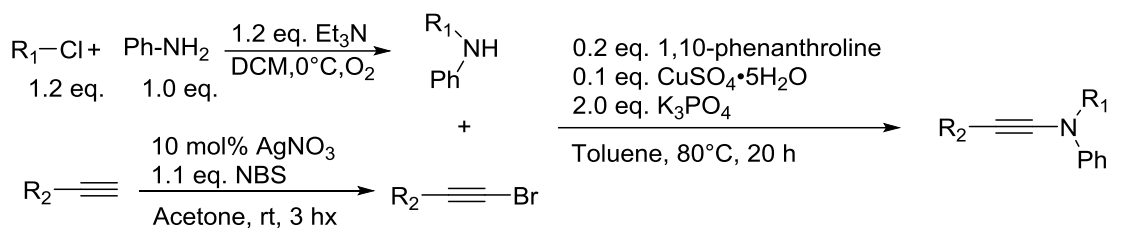

a) To a solution of $\mathrm{Et}_{3} \mathrm{~N}(1.7 \mathrm{~mL}, 12.0 \mathrm{mmol})$ and Aniline $(0.9 \mathrm{~mL}, 10.0 \mathrm{mmol})$ in $\mathrm{CH}_{2} \mathrm{Cl}_{2}(30 \mathrm{~mL})$ stirred at $0{ }^{\circ} \mathrm{C}$ was added acyl chloride $(12.0 \mathrm{mmol})$ portionwise. The resulting solution was stirred at $0{ }^{\circ} \mathrm{C}$ for $30 \mathrm{~min}$. and then was extracted with $\mathrm{CH}_{2} \mathrm{Cl}_{2}$. The organic extracts were dried over $\mathrm{Na}_{2} \mathrm{SO}_{4}$, filtered, and concentrated in vacuo. The product can be used without further purification, but if desired, it can be purified by silica gel flash column chromatography $(\mathrm{EtOAc} /$ hexane $=1: 20)$ to afford amide.

b) To a solution of alkyne (1.0 equiv, $10 \mathrm{mmol})$ in acetone $(0.1 \mathrm{M}, 100 \mathrm{~mL})$ was added NBS (1.1 equiv, $11 \mathrm{mmol})$ and $\mathrm{AgNO}_{3}(10 \mathrm{~mol} \%, 1 \mathrm{mmol})$ the resulting mixture was stirred under nitrogen for $3 \mathrm{~h}$ at room temperature. After removing excess acetone, the reaction was quenched with water, and the organic layer was extracted with pentane (30 $\mathrm{mL} \times 3$ ), organic layer was dried over $\mathrm{Na}_{2} \mathrm{SO}_{4}$, the organic solvent was evaporated, and the residue was subjected to column chromatography on silica gel (petroleum ether) to obtain pure colorless oil of acetylene bromide.

c) To a dried flask was added amide (1.0 equiv, $5 \mathrm{mmol}), \mathrm{CuSO}_{4} \cdot 5 \mathrm{H}_{2} \mathrm{O}(10 \mathrm{~mol} \%, 0.5$ mmol), 1,10-phenanthroline (20 mol\%, $1 \mathrm{mmol}$ ) and $\mathrm{K}_{3} \mathrm{PO}_{4}(2.0$ equiv, $10 \mathrm{mmol})$, the mixture was subsequently treated with anhydrous toluene $(0.1 \mathrm{M}, 50 \mathrm{~mL})$ and acetylene bromide (1.2 equiv, $6 \mathrm{mmol})$. The reaction mixture was caped under a blanket of nitrogen and heated in an oil bath at $70-80{ }^{\circ} \mathrm{C}$ for $20 \mathrm{~h}$. After complete conversion of starting material, the reaction mixture was cooled to room temperature, filtered through 
Celite, and concentrated in vacuo. The methanol was removed and the residue obtained was purified by column chromatography on silica gel (petroleum ether : ethyl acetate = 10: 1) to afford the desired product. 


\subsection{General procedure for the preparation of Vinyl sulfonates $(4 b, 4 c)$}

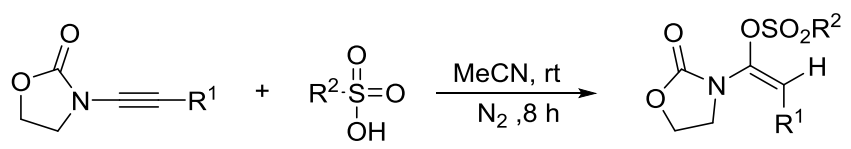

To a mixture of 1 (1.0 equiv, $1.0 \mathrm{mmol}), 2$ ( 2 equiv, $2.0 \mathrm{mmol}$ ) in a round bottom flask were added $\mathrm{MeCN}(0.1 \mathrm{M}, 10 \mathrm{~mL})$. The resulting mixture was stirred under nitrogen for $8 \mathrm{~h}$ at room temperature. Upon completion, the reaction mixture was cooled to $\mathrm{rt}$, filtered through Celite, and concentrated in vacuo. The crude residue was purified by silica gel flash column chromatography to afford $\mathbf{4}$ as white soild.

\subsection{Reaction Condition Optimization}

Table S1: Solvent screening ${ }^{[a]}$

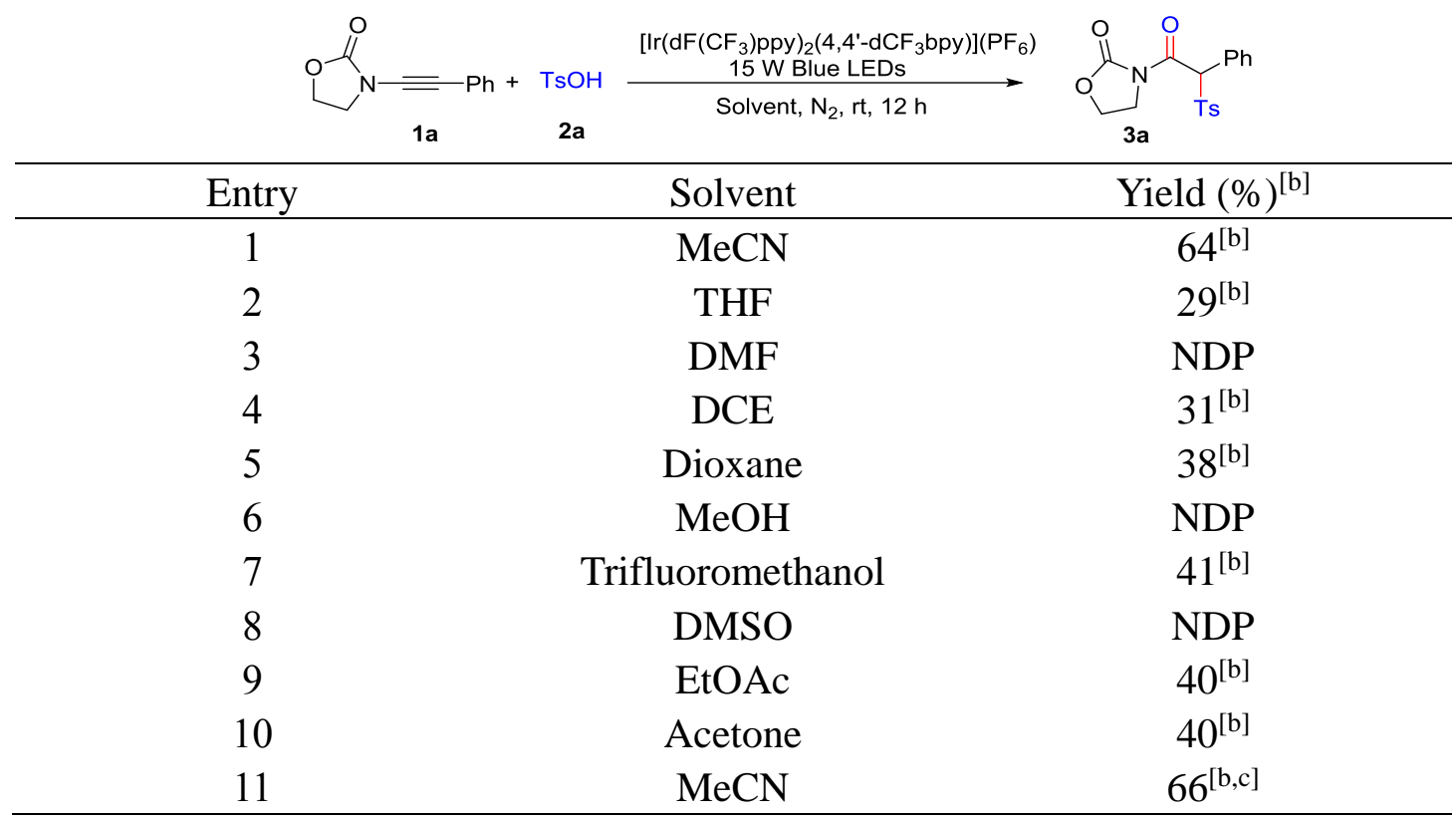

${ }^{[a]}$ Unless otherwise noted, the reactions were carried out with $1 \mathrm{a}(0.1 \mathrm{mmol}), \mathbf{2 a}(0.1 \mathrm{mmol})$, photocatalyst $(0.2 \mathrm{~mol} \%)$ in solvent $(1.0 \mathrm{~mL})$, irradiating with $15 \mathrm{~W}$ Blue LEDs under $\mathrm{N}_{2}$ atmosphere at room temperature for 12 hours. ${ }^{[b]}$ Yields were determined by ${ }^{1} \mathrm{H}$ NMR using $\mathrm{C}_{2} \mathrm{H}_{2} \mathrm{Cl}_{4}$ as internal standard. ${ }^{[c]}$ The solvent is extra dry, with molecular sieves. $\mathrm{C}_{2} \mathrm{H}_{2} \mathrm{Cl}_{4}, 1,1,2,2-$ Tetrachloroethane THF, tetrahydrofuran. DMF, $N, N$-dimethylforamide. DMSO, dimethyl sulfoxide. DCE, -dimethoxyethane. NDP, no desire product.

Table S2: Catalyst screening ${ }^{[a]}$ 


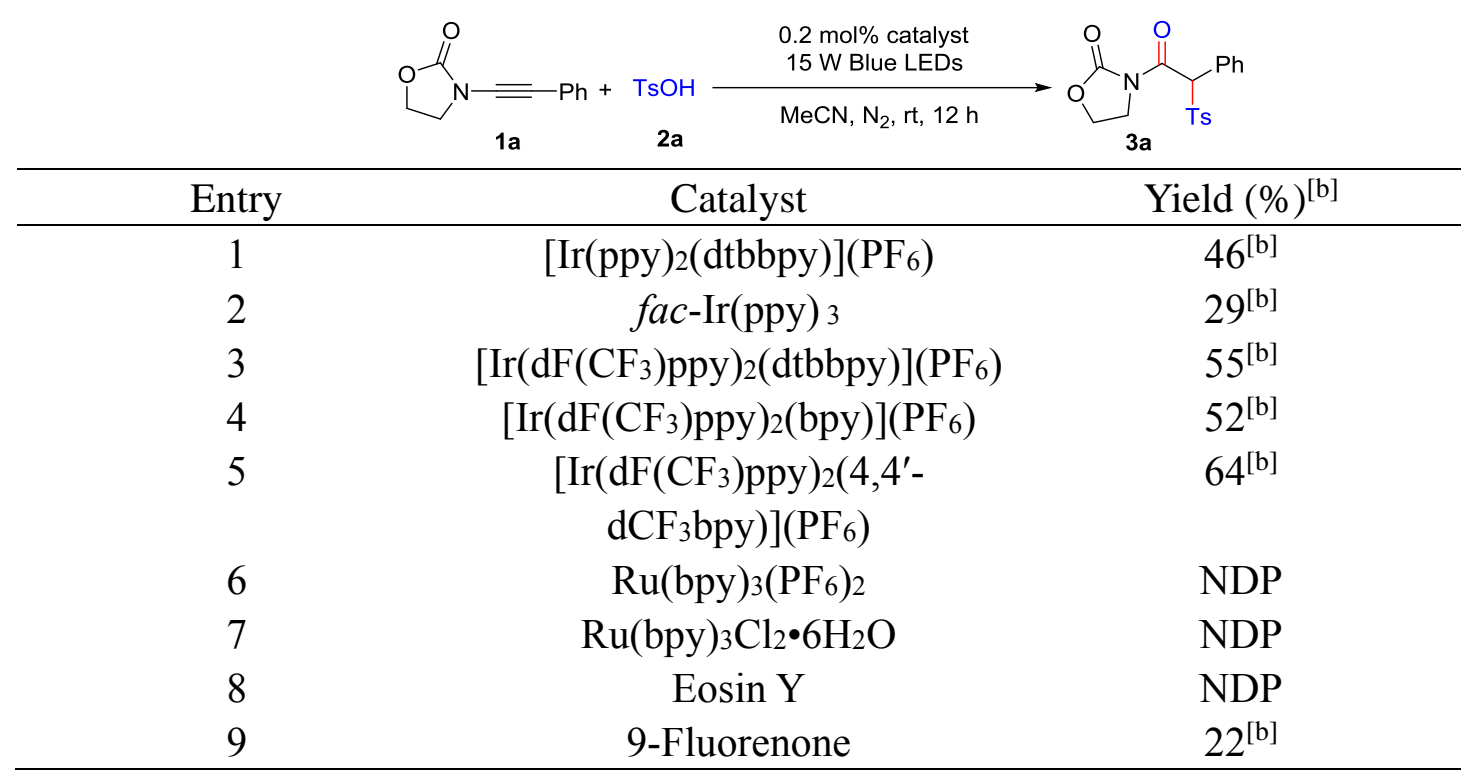

${ }^{[a]}$ Unless otherwise noted, the reactions were carried out with $\mathbf{1 a}(0.1 \mathrm{mmol}), \mathbf{2 a}(0.1 \mathrm{mmol})$, photocatalyst $(0.2 \mathrm{~mol} \%)$ in $\mathrm{MeCN}(1.0 \mathrm{~mL})$, irradiating with $15 \mathrm{~W}$ Blue LEDs under $\mathrm{N}_{2}$ atmosphere at room temperature for 12 hours. ${ }^{[b]}$ Yields were determined by ${ }^{1} \mathrm{H}$ NMR using $\mathrm{C}_{2} \mathrm{H}_{2} \mathrm{Cl}_{4}$ as internal standard.

Table S3: The influence of Sulfonic acid stoichiometry ${ }^{[a]}$

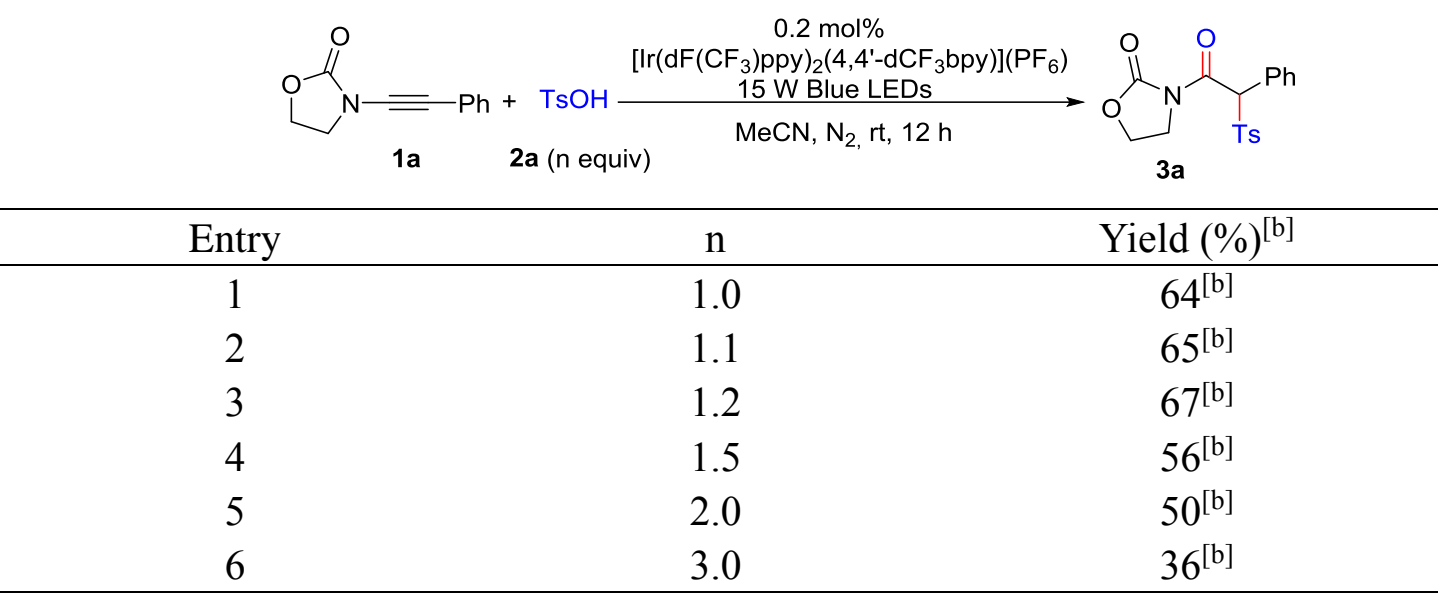

${ }^{[a]}$ Unless otherwise noted, the reactions were carried out with 1 a $(0.1 \mathrm{mmol}), \mathbf{2 a}$ (n equiv), photocatalyst $(0.2 \mathrm{~mol} \%)$ in $\mathrm{MeCN}(1.0 \mathrm{~mL})$, irradiating with $15 \mathrm{~W}$ Blue LEDs under $\mathrm{N}_{2}$ atmosphere at room temperature for 12 hours. ${ }^{[b]}$ Yields were determined by ${ }^{1} \mathrm{H}$ NMR using $\mathrm{C}_{2} \mathrm{H}_{2} \mathrm{Cl}_{4}$ as internal standard.

Table S4: Light source screening ${ }^{[a]}$ 


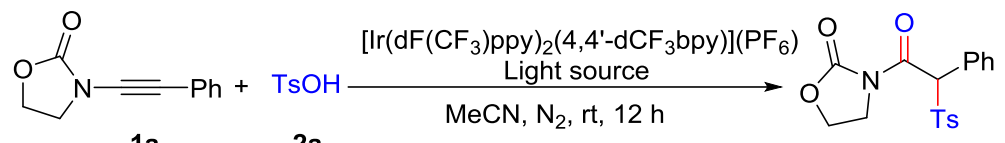

1a

$2 a$

$3 a$

\begin{tabular}{ccc}
\hline Entry & Light source & Yield $(\%)^{[\mathbf{b}]}$ \\
\hline 1 & Green & NDP \\
2 & Yellow & NDP \\
3 & Red & NDP \\
4 & White & $52^{[\mathbf{b}]}$ \\
5 & Blue & $67^{[\mathbf{b}]}$ \\
6 & Purple & $64^{[\mathbf{b}]}$ \\
\hline
\end{tabular}

${ }^{[a]}$ Unless otherwise noted, the reactions were carried out with 1a $(0.1 \mathrm{mmol}), \mathbf{2 a}$ (n equiv), photocatalyst $(0.2 \mathrm{~mol} \%)$ in $\mathrm{MeCN}(1.0 \mathrm{~mL})$, irradiating with $15 \mathrm{~W}$ LEDs under $\mathrm{N}_{2}$ atmosphere at room temperature for 12 hours. ${ }^{[b]}$ Yields were determined by ${ }^{1} \mathrm{H}$ NMR using $\mathrm{C}_{2} \mathrm{H}_{2} \mathrm{Cl}_{4}$ as internal standard.

Table S5: System Concentration Screening ${ }^{[a]}$
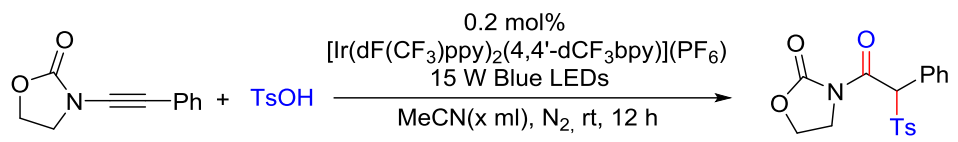

$1 \mathrm{a}$

2a

$3 a$

\begin{tabular}{cccc}
\hline Entry & $\begin{array}{c}\text { System } \\
\text { concentration }\end{array}$ & $\mathrm{x}(\mathrm{mL})$ & Yield $(\%)^{[\mathrm{b}]}$ \\
\hline 1 & $0.1 \mathrm{M}$ & 1.0 & $64^{[\mathrm{b}]}$ \\
2 & $0.2 \mathrm{M}$ & 0.5 & $69^{[\mathrm{b}]}$ \\
3 & $0.25 \mathrm{M}$ & 0.4 & $79^{[\mathrm{b}]}$ \\
4 & $0.3 \mathrm{M}$ & 0.33 & $80^{[\mathrm{b}]}(78)^{[\mathrm{c}]}$ \\
5 & $0.4 \mathrm{M}$ & 0.25 & $50^{[\mathrm{b}]}$ \\
\hline
\end{tabular}

${ }^{[a]}$ Unless otherwise noted, the reactions were carried out with $1 \mathbf{a}(0.1 \mathrm{mmol}), \mathbf{2 a}(0.1 \mathrm{mmol})$, photocatalyst $(0.2 \mathrm{~mol} \%)$ in MeCN, irradiating with $15 \mathrm{~W}$ Blue LEDs under $\mathrm{N}_{2}$ atmosphere at room temperature for 12 hours. ${ }^{[b]}$ Yields were determined by ${ }^{1} \mathrm{H}$ NMR using $\mathrm{C}_{2} \mathrm{H}_{2} \mathrm{Cl}_{4}$ as internal standard. ${ }^{[c]}$ with figures in the parentheses being the isolated yields. 


\subsection{General Procedure for Oxo-sulfonylation of Ynamides with}

\section{Sulfonic Acids}

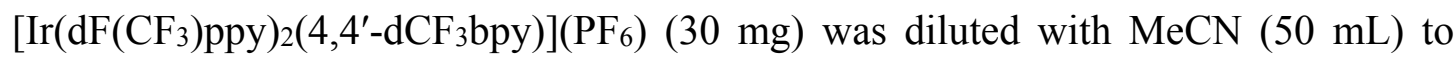
form a $0.2 \mathrm{~mol} \%$ solution of photocatalyst. 3-(phenylethynyl)oxazolidin-2-one 1 (0.1 mmol, 1.0 equiv), $2 \mathrm{TsOH}$ ( $0.12 \mathrm{mmol}, 1.2$ equiv) were introduced to a $10 \mathrm{~mL}$ screw cap vial equipped with a magnetic stir bar. $0.6 \mathrm{mg} / \mathrm{mL}$ solution of photocatalyst $(0.33$ $\mathrm{mL}$ ) was added under $\mathrm{N}_{2}$ atmosphere. The vial was sealed, and the reaction was then irradiated using $15 \mathrm{~W}$ blue LEDs at $\mathrm{rt}$ for $12 \mathrm{~h}$. When the reaction was observed to be completed by TLC, the reaction mixture was filtered through silica gel, which was washed with EtOAc. The filtrate was concentrated under reduced pressure and purified by column chromatography on silica gel (petroleum ether: ethyl acetate $=13: 7$ ) to give 3. 


\section{Mechanistic Study}

\subsection{Cyclic Voltammetry}

Cyclic voltammograms were taken on a $\mathrm{CH}$ Instruments $600 \mathrm{E}$ potentiostat using a glassy carbon working electrode, a saturated calomel reference electrode (SCE), and a Pt mesh counter electrode. The $\mathrm{pH}$ was not adjusted and voltammograms were taken at room temperature in a $100 \mathrm{mM} \mathrm{MeCN}$ solution of tetrabutylammonium hexafluorophosphate containing $10 \mathrm{mM}$ of the designated substance unless otherwise specified. The scan rate was $100 \mathrm{mV} / \mathrm{s}$. Based on the reduction potentials of intermediate $4 \mathbf{a}\left(E_{1 / 2}{ }^{\text {red }}=-0.94 \mathrm{~V}\right)($ Figure $\mathrm{S} 1)$ and $\left[\operatorname{Ir}\left(\mathrm{dF}\left(\mathrm{CF}_{3}\right) \text { ppy }\right)_{2}\left(4,4^{\prime}-\mathrm{dCF}_{3} \text { bpy }\right)^{+}\right.$ $\left(E_{1 / 2}\left({ }^{*} \mathrm{Ir}^{\mathrm{III}} / \mathrm{Ir}^{\mathrm{IV}}\right)=-0.51 \mathrm{~V} \text { vs SCE}\right)^{[11]}$, the spectrum showed reduction of intermediate by the excited state * $\mathrm{Ir}^{\mathrm{III}}$ was thermodynamically unfavorable.

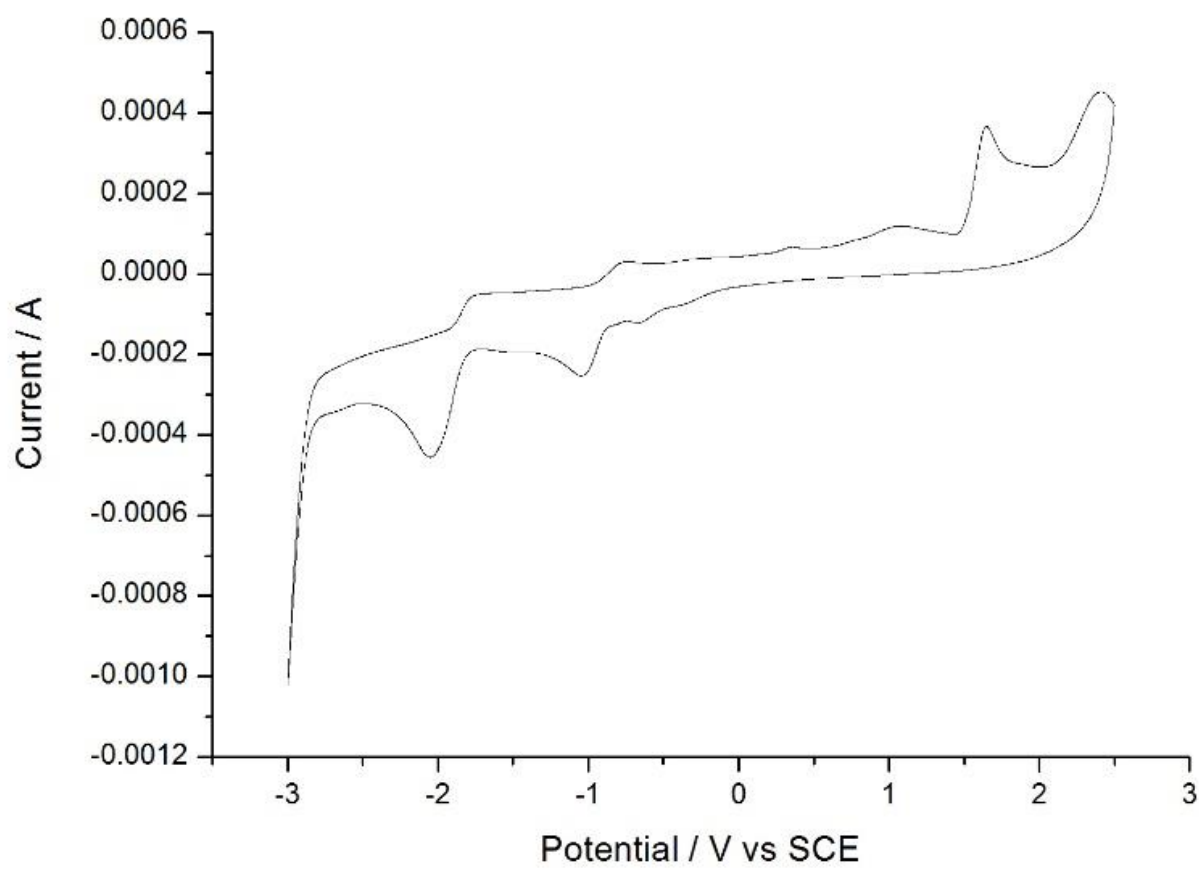

Figure S1. Cyclic voltammogram of intermediate 4a.

\subsection{Emission quenching experiments (Stern- Volmer studies)}

Emission intensities were recorded using PerkinElemer LS 55 Fluorescence Spectrometer for all experiments. All $\left[\operatorname{Ir}\left(\mathrm{dF}\left(\mathrm{CF}_{3}\right) \text { ppy }\right)_{2}(\mathrm{dtbbpy})\right]\left(\mathrm{PF}_{6}\right)$ solutions $(0.001$ $\mathrm{mM}$ ) were excited at $390 \mathrm{~nm}$ and the emission intensity at $474 \mathrm{~nm}$ was collected at 
room temperature under an inert atmosphere. Samples were prepared by adding solutions of photocatalyst, quencher, and $\mathrm{MeCN}$ to obtain a total volume of $3.0 \mathrm{~mL}$ in glove box. The sample was shaken for $1.0 \mathrm{~min}$ and then the emission of the sample was collected. All the experiments are run in duplicate. The data show that 4a was competent at quenching the excited state of the photocatalyst, while $\mathbf{1 a}$ and $\mathbf{2}$ was show unable to quench this excited state photocatalyst.

\section{Constant Iridium, Varied 4a.}

\begin{tabular}{|c|c|c|c|c|c|}
\hline \multicolumn{3}{|c|}{ Species } & \multicolumn{3}{|c|}{ Concentration (mM) } \\
\hline \multicolumn{3}{|c|}{ 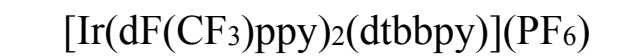 } & \multicolumn{3}{|c|}{0.001} \\
\hline \multicolumn{3}{|c|}{$\mathbf{4 a}$} & \multicolumn{3}{|c|}{ Varied } \\
\hline$[4 \mathbf{a}](\mathrm{mM})$ & Scan 1 & Scan 2 & Scan 3 & Average & $\mathrm{I}_{0} / \mathrm{I}$ \\
\hline 0 & 283.91 & 283.85 & 282.69 & 283.48 & 1.00 \\
\hline 0.25 & 198.05 & 197.98 & 196.33 & 197.46 & 1.44 \\
\hline 0.5 & 164.95 & 165.18 & 164.50 & 164.88 & 1.72 \\
\hline 1.0 & 128.79 & 129.96 & 128.96 & 129.24 & 2.20 \\
\hline 1.5 & 99.62 & 100.24 & 99.87 & 99.91 & 2.84 \\
\hline
\end{tabular}

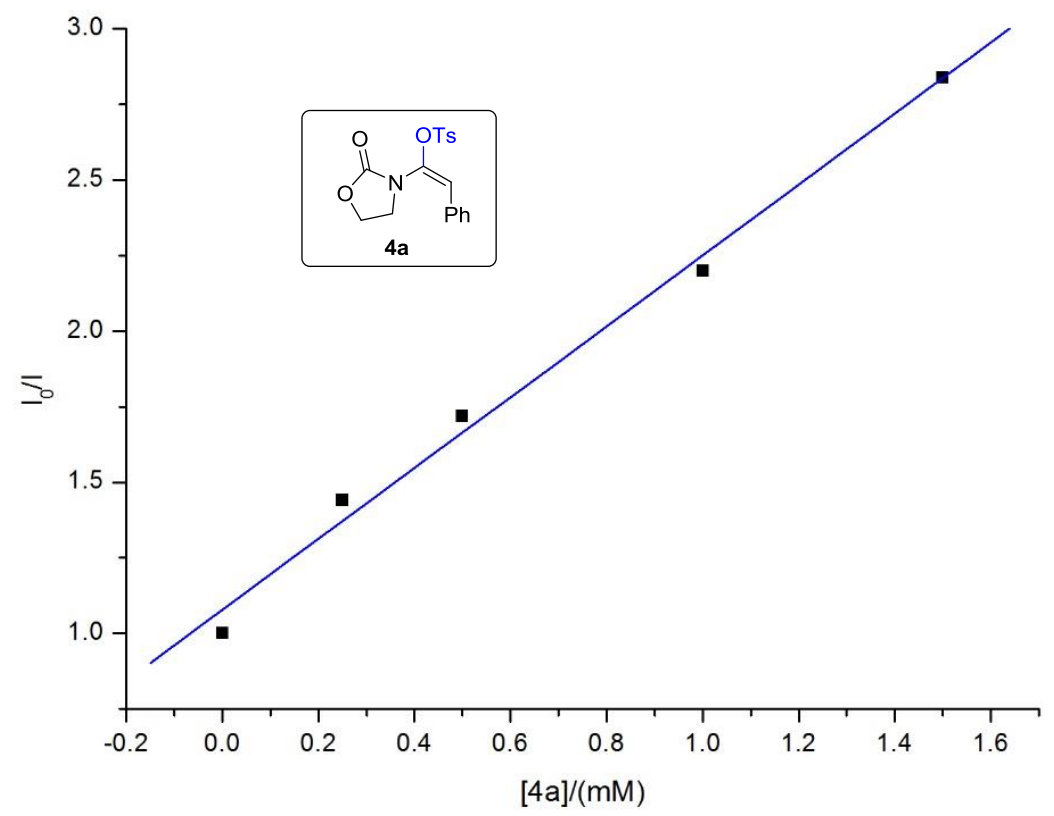




\section{Constant Iridium, Varied 1a.}

\begin{tabular}{cccccc}
\hline \multicolumn{2}{c}{ Species } & \multicolumn{3}{c}{ Concentration $(\mathbf{m M})$} \\
\hline \multicolumn{2}{c}{$\left[\operatorname{Ir}\left(\mathrm{dF}\left(\mathrm{CF}_{3}\right) \text { ppy }\right)_{2}(\mathrm{dtbbpy})\right]\left(\mathrm{PF}_{6}\right)$} & \multicolumn{3}{c}{$\begin{array}{c}0.001 \\
\text { 1a }\end{array}$} \\
\hline \multicolumn{3}{c}{ Varied } \\
\hline$[\mathbf{1 a}](\mathrm{mM})$ & Scan 1 & Scan 2 & Scan 3 & Average & $\mathrm{I}_{0} / \mathrm{I}$ \\
\hline 0 & 287.81 & 287.63 & 287.75 & 287,73 & 1.00 \\
0.25 & 290.02 & 289.34 & 288.93 & 289.43 & 0.99 \\
0.5 & 292.30 & 291.80 & 291.08 & 291.73 & 0.99 \\
1.0 & 289.51 & 289.52 & 291.27 & 290.10 & 0.99 \\
1.5 & 286.50 & 286.50 & 286.50 & 286.50 & 1.00 \\
\hline
\end{tabular}

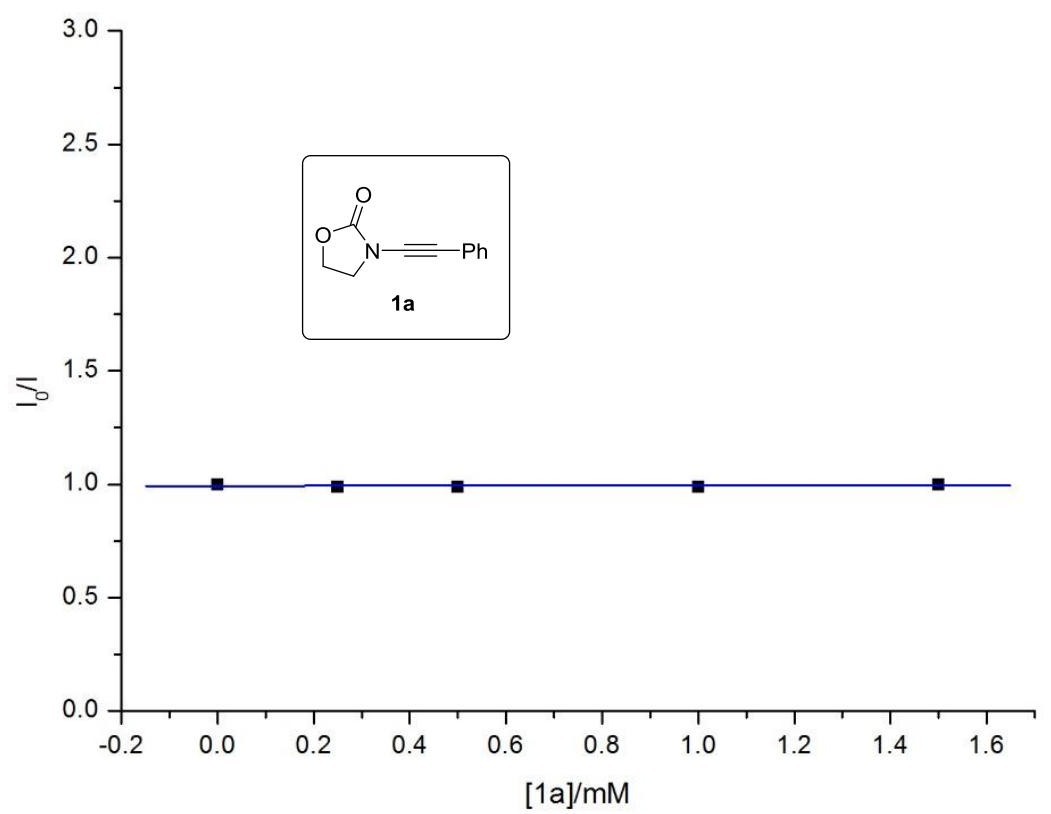

\section{Constant Iridium, Varied 2a.}

\begin{tabular}{|c|c|c|c|c|c|}
\hline \multicolumn{3}{|c|}{ Species } & \multicolumn{3}{|c|}{ Concentration (mM) } \\
\hline \multicolumn{3}{|c|}{$\left.\left.\left[\operatorname{Ir}\left(\mathrm{dF}_{(\mathrm{CF}}\right) \mathrm{ppy}\right)_{2} \mathrm{dtbbpy}\right)\right]\left(\mathrm{PF}_{6}\right)$} & \multicolumn{3}{|c|}{0.001} \\
\hline \multicolumn{3}{|c|}{$2 \mathbf{a}$} & \multicolumn{3}{|c|}{ Varied } \\
\hline$[2](\mathrm{mM})$ & Scan 1 & Scan 2 & Scan 3 & Average & $\mathrm{I} 0 / \mathrm{I}$ \\
\hline 0 & 281.20 & 281.32 & 281.00 & 281.17 & 1.00 \\
\hline 0.25 & 280.89 & 281.02 & 280.75 & 280.89 & 1.00 \\
\hline 0.5 & 280.69 & 280.98 & 280.75 & 280.81 & 1.00 \\
\hline 1.0 & 279.56 & 279.28 & 279.06 & 279.30 & 1.01 \\
\hline 1.5 & 280.85 & 280.53 & 280.22 & 280.53 & 1.00 \\
\hline
\end{tabular}




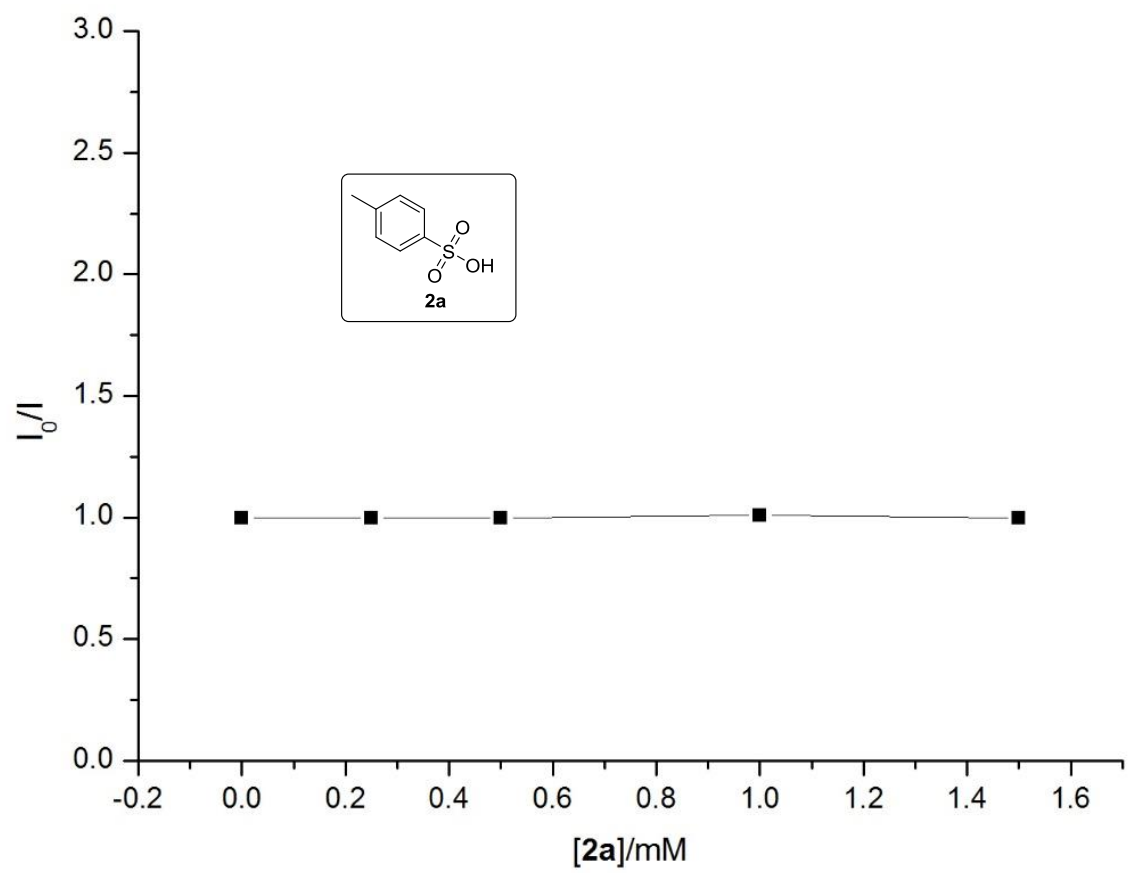

\subsection{Light on/off experiment:}

$\left[\operatorname{Ir}\left(\mathrm{dF}\left(\mathrm{CF}_{3}\right) \text { ppy }\right)_{2}\left(4,4^{\prime}-\mathrm{dCF}_{3}\right.\right.$ bpy $\left.)\right]\left(\mathrm{PF}_{6}\right)(30 \mathrm{mg})$ was diluted with $\mathrm{MeCN}(50 \mathrm{~mL})$ to form a 0.2 mol\% solution of photocatalyst. 3-((4(trifluoromethyl)phenyl)ethynyl)oxazolidin-2-one $\mathbf{1 i}(0.1 \mathrm{mmol}, 1.0$ equiv), 2a TsOH (0.12 mmol, 1.2 equiv) were introduced to a $10 \mathrm{~mL}$ screw cap vial equipped with a magnetic stir bar. $0.2 \mathrm{~mol} \%$ solution of photocatalyst $(0.33 \mathrm{~mL})$ was added under $\mathrm{N}_{2}$ atmosphere. This reaction was performed using alternating intervals of light and dark. The reaction profile is shown below and the yield of product $3 \mathbf{i}$ was determined by ${ }^{1} \mathrm{H}$ NMR (1,1,2,2-tetrechloroethane was used as an internal standard). These results indicated that continuous irradiation with light was essential for promoting the reaction.

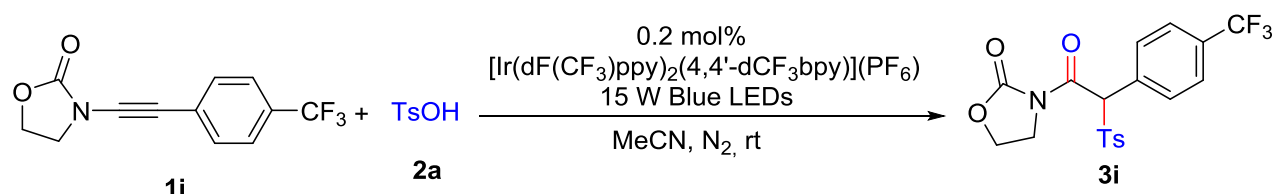




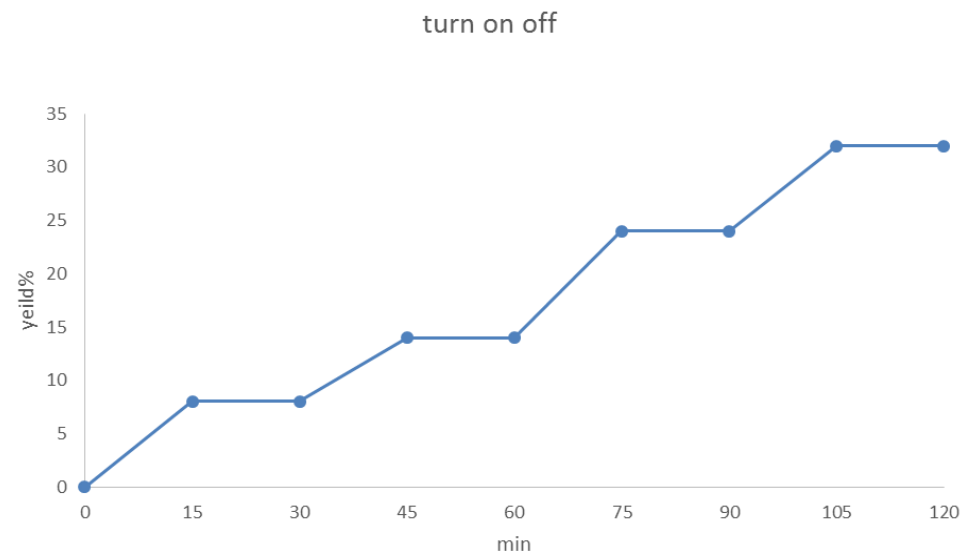

Figure S2. Light on/off experiment of reaction

\subsection{Gram-Scale Experiments.}

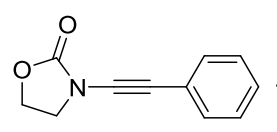

1a

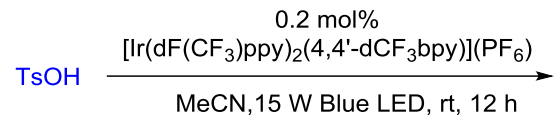

$2 \mathrm{a}$

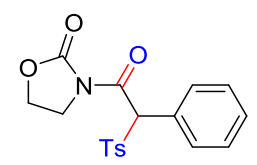

3a $(70 \%, 1.26 \mathrm{~g})$

$\left[\operatorname{Ir}\left(\mathrm{dF}_{(\mathrm{CF}}\right) \text { ppy }\right)_{2}\left(4,4^{\prime}-\mathrm{dCF}_{3}\right.$ bpy) $]\left(\mathrm{PF}_{6}\right)(30 \mathrm{mg})$ was diluted with $\mathrm{MeCN}(50 \mathrm{~mL})$ to form a $0.2 \mathrm{~mol} \%$ solution of photocatalyst. 3-(phenylethynyl)oxazolidin-2-one $\mathbf{1 a}$ (5 mmol, 1.0 equiv), TsOH 2a (6 mmol, 1.2 equiv) were introduced to an over-dried 50 $\mathrm{mL}$ schlenk tube equipped with a magnetic stir bar. $0.2 \mathrm{~mol} \%$ solution of photocatalyst (16.5 mL) was added under $\mathrm{N}_{2}$ atmosphere. The vial was sealed, and the reaction was then irradiated using $15 \mathrm{~W}$ blue LEDs at $\mathrm{rt}$ for $12 \mathrm{~h}$. When the reaction was observed to be complete by TLC, the reaction mixture was filtered through silica gel, which was washed with EtOAc. The filtrate was concentrated under reduced pressure and purified by silica gel column chromatography (petroleum ether/ethyl acetate $=3: 2$ ) to afford $1.26 \mathrm{~g} \mathrm{3a}$ in $70 \%$ yield. 


\subsection{The Crossover Experiments}

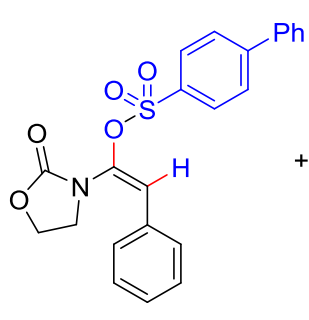

4b $0.1 \mathrm{mmol}$

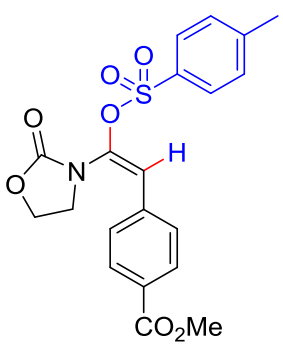

4c $0.1 \mathrm{mmol}$
$0.2 \mathrm{~mol} \%$

$\left[\operatorname{lr}\left(\mathrm{dF}\left(\mathrm{CF}_{3}\right) \mathrm{ppy}\right)_{2}\left(4,4^{\prime}-\mathrm{dCF}_{3} \mathrm{bpy}\right)\right]\left(\mathrm{PF}_{6}\right)$

MeCN, $15 \mathrm{~W}$ Blue LED, rt, $12 \mathrm{~h}$

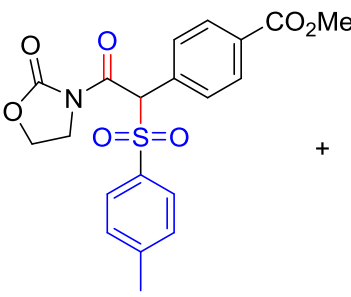

$3 \mathbf{j}$

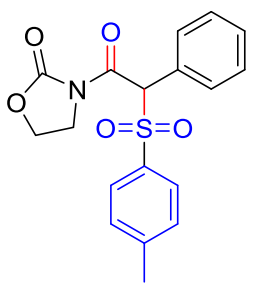

3a

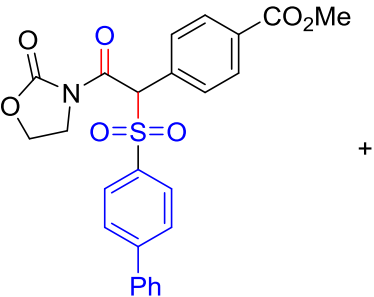

3ar

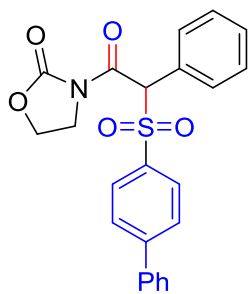

3an

3j:3a:3ar:3an $=10: 17: 10: 18$

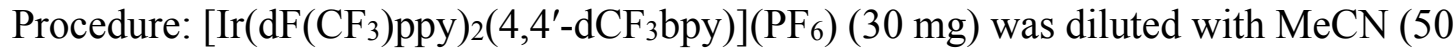
$\mathrm{mL}$ ) to form a $0.2 \mathrm{~mol} \%$ solution of photocatalyst. The cross over experiment has been carried out by subjecting an equimolar amount of $\mathbf{4 b}(0.1 \mathrm{mmol})$ and $\mathbf{4 c}(0.1 \mathrm{mmol})$ were introduced to an over-dried $10 \mathrm{~mL}$ schlenk tube equipped with a magnetic stir bar. $0.2 \mathrm{~mol} \%$ solution of photocatalyst $(0.33 \mathrm{~mL})$ was added under $\mathrm{N}_{2}$ atmosphere. The vial was sealed, and the reaction was then irradiated using $15 \mathrm{~W}$ blue LEDs at $\mathrm{rt}$ for $12 \mathrm{~h}$. These products were detected by crude ${ }^{1} \mathrm{H}$ NMR. 
둥ำ

1

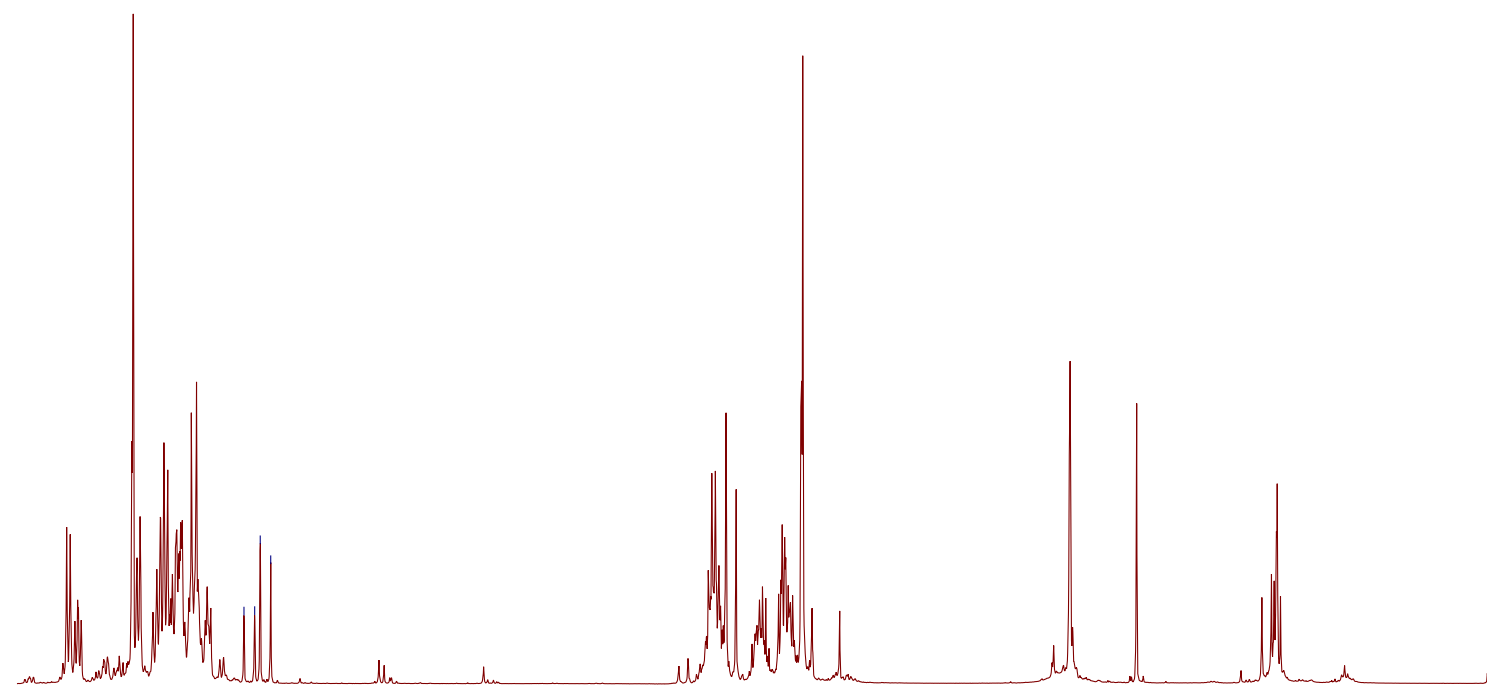

80

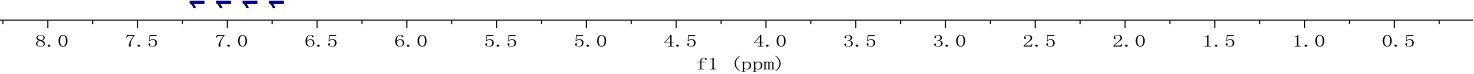

זָ

\begin{tabular}{ll}
$\stackrel{2}{0}$ & \multirow{2}{0}{} \\
0 & i
\end{tabular}

$\underset{\substack{0 \\ 0 \\ i}}{\infty}$

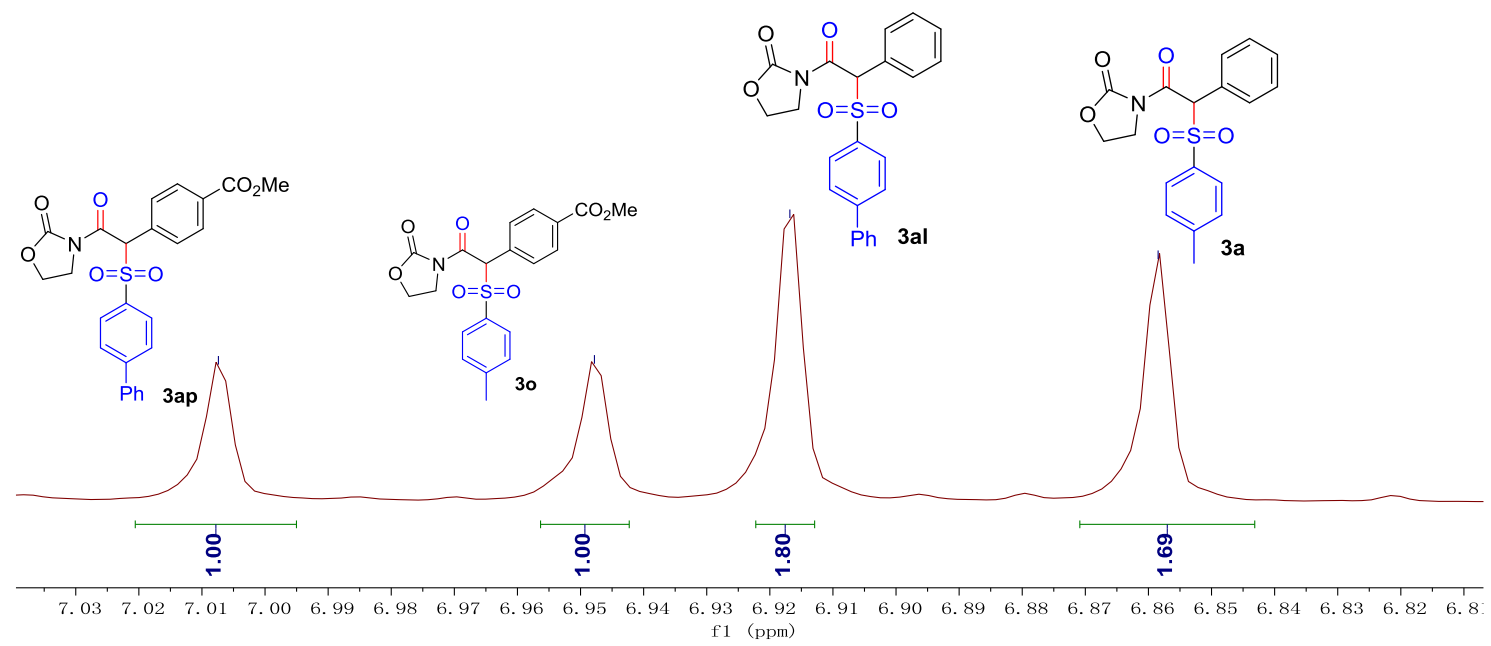




\subsection{Control Experiments}

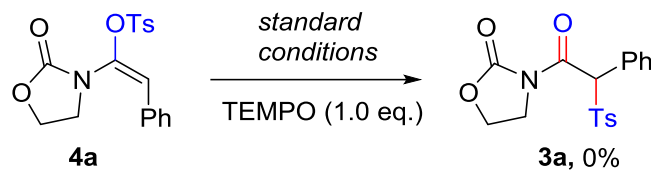

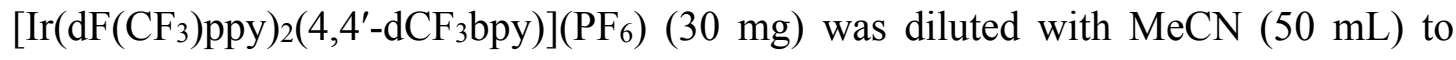
form a $0.2 \mathrm{~mol} \%$ solution of photocatalyst. The experiment has been carried out by $\mathbf{4 a}$ $(0.1 \mathrm{mmol})$ and TEMPO $(0.1 \mathrm{mmol})$ were introduced to an over-dried $10 \mathrm{~mL}$ schlenk tube equipped with a magnetic stir bar. $0.2 \mathrm{~mol} \%$ solution of photocatalyst $(0.67 \mathrm{~mL})$ was added under $\mathrm{N}_{2}$ atmosphere. The vial was sealed, and the reaction was then irradiated using $15 \mathrm{~W}$ blue LEDs at $\mathrm{rt}$ for $12 \mathrm{~h}$. These products were detected by crude ${ }^{1} \mathrm{H}$ NMR.

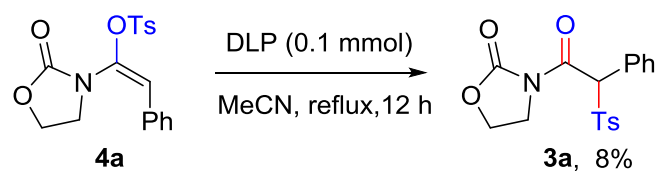

The experiment has been carried out by $\mathbf{4 a}(0.1 \mathrm{mmol})$ and dilauroyl peroxide (DLP) $(0.1 \mathrm{mmol})$ were introduced to an over-dried $10 \mathrm{~mL}$ schlenk tube equipped with a magnetic stir bar. $\mathrm{MeCN}(0.33 \mathrm{~mL})$ was added under $\mathrm{N}_{2}$ atmosphere. The vial was sealed, and the reaction was then reflux for $12 \mathrm{~h}$ at $80{ }^{\circ} \mathrm{C}$. These products were detected by crude ${ }^{1} \mathrm{H}$ NMR.

\subsection{UV Sensitizer Experiments}

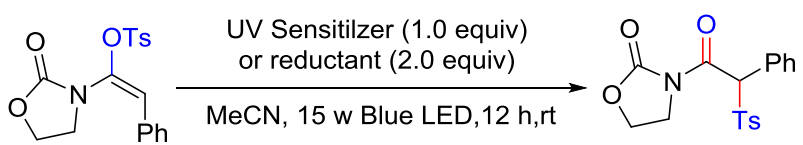

4a

$3 a$

\begin{tabular}{cccc}
\hline Entry & UV Sensitizer & Reductant & yield \\
\hline 1 & none & Mn powder & $0 \%$ \\
2 & benzil & none & $56 \%$ \\
\hline
\end{tabular}

The following procedure was employed for the experiments: To an over-dried $10 \mathrm{~mL}$ schlenk tube equipped with a magnetic stir bar were added $\mathbf{4 a}(36.0 \mathrm{mg}, 0.1 \mathrm{mmol}, 1.0$ equiv.), UV sensitizer ( $0.1 \mathrm{mmol}, 1.0$ equiv) or Mn Powder (dried at $120^{\circ} \mathrm{C}$ overnight, 
$11.0 \mathrm{mg}, 0.2 \mathrm{mmol}, 2.0$ equiv). $\mathrm{MeCN}(0.33 \mathrm{~mL})$ was added under $\mathrm{N}_{2}$ atmosphere. This mixture was then irradiated using $15 \mathrm{~W}$ blue LEDs at $\mathrm{rt}$ for $12 \mathrm{~h}$. These products were detected by crude ${ }^{1} \mathrm{HNMR}$.

When the UV Sensitizer possessing higher triplet energy than that of the $4 \mathbf{a}$ was employed, good yield of 3a was obtained, strongly suggesting that an energy transfer mechanism is indeed involved. Mn powder $\left(E^{\mathrm{o}}=-1.42 \mathrm{~V}\right.$ vs. SCE (saturated calomel electrode)), ${ }^{[12]}$ which was used as an external reductant (comparable to $\left[\operatorname{Ir}\left(\mathrm{dF}\left(\mathrm{CF}_{3}\right) \text { ppy }\right)_{2}\left(4,4^{\prime}-\mathrm{dCF}_{3} \text { bpy }\right)^{+}\left(E_{1 / 2}\left({ }^{*} \mathrm{Ir}^{\mathrm{III}} / \mathrm{Ir}^{\mathrm{IV}}\right)=-0.51 \mathrm{~V}\right.\right.$ vs $\left.\mathrm{SCE}\right),{ }^{[11]}$ failed to promote the desired reaction, again indicating that photoredox-mediated pathway was not operative for this transformation.

\subsection{Determination of the reaction quantum yield $(\Phi)$ :}

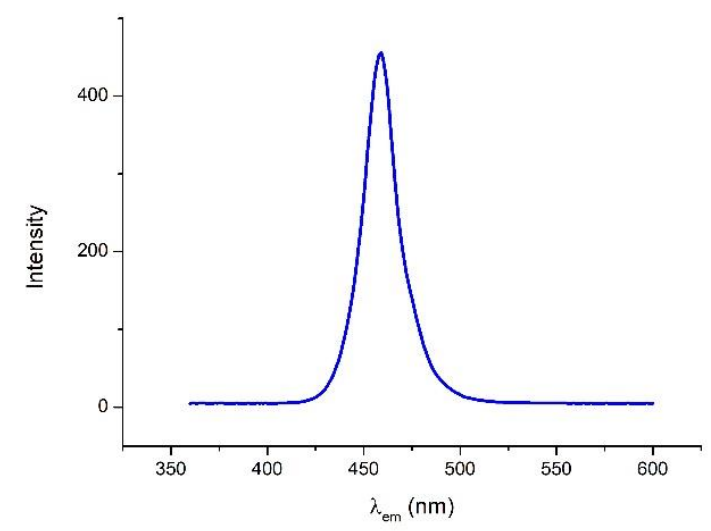

Figure S3. Emission spectrum of blue LED used for quantum yield experiments $\left(\lambda_{\max }=\right.$ $459 \mathrm{~nm})$. Recorded using a F-4600 FL Spectrophotometer.

\section{Determination of the light intensity at $459 \mathrm{~nm}$ :}

According to the procedure of Yoon, ${ }^{[13]}$ the photon flux of the blue LED $\left(\lambda_{\max }=459\right.$ $\mathrm{nm}$ ) was determined by standard ferrioxalate actinometry. ${ }^{[14,15,16]} 2.21 \mathrm{~g}$ of Potassium ferrioxalate hydrate was dissolved in $30 \mathrm{~mL}$ of $0.05 \mathrm{M} \mathrm{H}_{2} \mathrm{SO}_{4}$ to prepare a $0.15 \mathrm{M}$ solution of ferrioxalate. A buffered solution of phenanthroline was prepared by dissolving $50 \mathrm{mg}$ of phenanthroline and $11.25 \mathrm{~g}$ of sodium acetate in $50 \mathrm{~mL}$ of $0.5 \mathrm{M}$ $\mathrm{H}_{2} \mathrm{SO}_{4}$. Both solutions were stored in the dark. To determine the photon flux of the blue LED, $2.0 \mathrm{~mL}$ of the ferrioxalate solution was placed in a $3 \mathrm{~mL}$ cuvette and irradiated for 90.0 seconds at $\lambda_{\max }=459 \mathrm{~nm}$ (Figure S3). After irradiation, $0.35 \mathrm{~mL}$ of the 
phenanthroline solution was added to the cuvette. The solution was then allowed to rest for $1 \mathrm{~h}$ to allow the ferrous ions to completely coordinate to the phenanthroline. The absorbance of the solution was measured at $510 \mathrm{~nm}$. A non-irradiated sample was also prepared and the absorbance at $510 \mathrm{~nm}$ measured. Conversion was calculated using eq. 1 .

$$
\text { mol } \mathrm{Fe}^{2+}=\frac{\mathrm{V} \cdot \Delta \mathrm{A}}{\mathrm{l} \cdot \varepsilon}
$$

Where $\mathrm{V}$ is the total volume $(0.00235 \mathrm{~L})$ of the solution after addition of phenanthroline, $\Delta \mathrm{A}$ is the difference in absorbance at $510 \mathrm{~nm}$ between the irradiated and non-irradiated solutions, 1 is the path length $(1.000 \mathrm{~cm})$, and $\varepsilon$ is the molar absorptivity at $510 \mathrm{~nm}$ $\left(11,100 \mathrm{~L} \mathrm{~mol}^{-1} \mathrm{~cm}^{-1}\right)$. The photon flux can be calculated using eq 2 .

$$
\text { photon flux }=\frac{\text { mol } \mathrm{Fe}^{2+}}{\Phi \cdot t \cdot f}
$$

Where $\Phi$ is the quantum yield for the ferrioxalate actinometer $(0.845$ for a $0.15 \mathrm{M}$ solution at $\lambda=459 \mathrm{~nm}),{ }^{[17]} \mathrm{t}$ is the time $(90.0 \mathrm{~s})$, and $\mathrm{f}$ is the fraction of light absorbed at $\lambda=459 \mathrm{~nm}(0.9538$ vide infra $)$. The photon flux was calculated to be $7.41 \times 10^{-9}$ einstein $\mathrm{s}^{-1}$.

\section{Determination of fraction of light absorbed at $459 \mathrm{~nm}$ for the ferrioxalate solution:}

The absorbance of the above ferrioxalate solution at $459 \mathrm{~nm}$ was measured to be 1.197.

The fraction of light absorbed (f) by this solution was calculated using eq 3, where A is the measured absorbance at $459 \mathrm{~nm}$.

$$
f=1-10^{-A}
$$

Sample calculation:

$\mathrm{mol} \mathrm{Fe}{ }^{2+}=(0.00235 \mathrm{~L} \times 2.51) /\left(1.000 \mathrm{~cm} \times 11100 \mathrm{~L} \mathrm{~mol}^{-1} \mathrm{~cm}^{-1}\right)=5.3 \times 10^{-7} \mathrm{~mol}$ $\mathrm{f}=1-10^{(-1.197)}=0.94$

photon flux $=5.3 \times 10^{-7} \mathrm{~mol} /(0.845 \times 90.0 \mathrm{~s} \times 0.94)=7.41 \times 10^{-9}$ einstein $\mathrm{s}^{-1}$ 


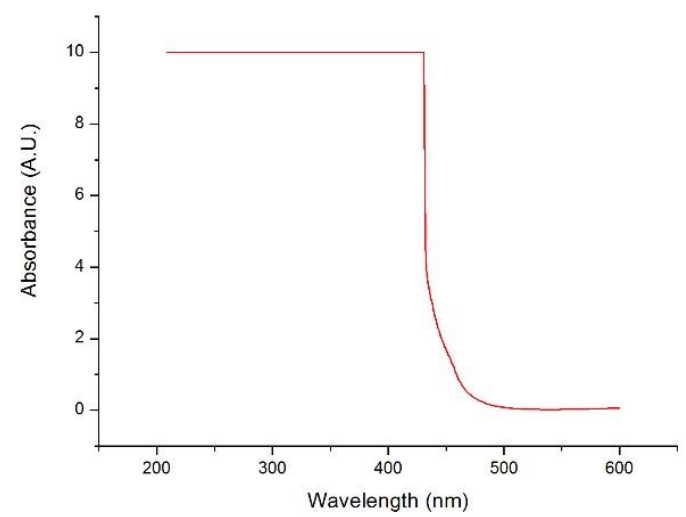

Figure S4. Absorbance of the ferrioxalate actinometer solution.

\section{Determination of the reaction quantum yield:}

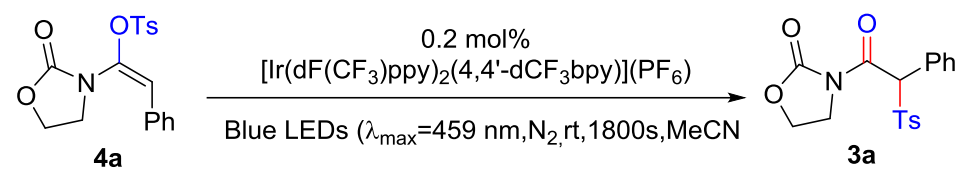

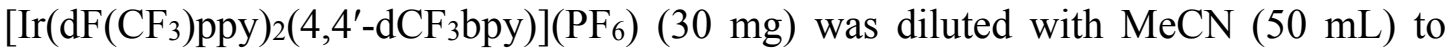
form a $0.2 \mathrm{~mol} \%$ solution of photocatalyst. To a $3 \mathrm{~mL}$ quartz cuvette with two sides taped over with electrical tape: 4 a $(216 \mathrm{mg}, 0.6 \mathrm{mmol}, 1$ equiv) and a small stir bar were added. The cuvette was pumped into the glovebox, where a solution of photocatalyst $(2.0 \mathrm{~mL})$,was introduced in sequence and the cuvette was sealed with a cap and septa. The vial was pumped out of the glovebox. The sample was stirred and irradiated for $1800 \mathrm{~s}(30 \mathrm{~min})$ at $\lambda_{\max }=459 \mathrm{~nm}$ at $\mathrm{rt}$. After irradiation, the yield of product 3 a was determined to be $12 \%\left(7.2 \times 10^{-5} \mathrm{~mol}\right.$ of $\left.\mathbf{3 a}\right)$ by ${ }^{1} \mathrm{H}$ NMR. The reaction quantum yield $(\Phi)$ was determined using eq 4 where the photon flux is $1.7 \times 10^{-10}$ einstein $\mathrm{s}^{-1}$ (determined by actinometry as described above), $\mathrm{t}$ is the reaction time (1800 $\mathrm{s})$ and $\mathrm{f}$ is the fraction of incident light absorbed by the reaction mixture, determined using eq 3. An absorbance of the reaction mixture at $459 \mathrm{~nm}$ was measured to be 0.340

$$
\Phi=\frac{\text { mol of product formed }}{\text { photon flux } \cdot \mathrm{t} \cdot \mathrm{f}}
$$

Sample quantum yield calculation

$\mathrm{f}=1-10^{(-0.34)}=0.54$

$\Phi=7.2 \times 10^{-5} \mathrm{~mol} /\left(7.41 \times 10^{-9}\right.$ einstein $\left.\mathrm{s}^{-1} \times 1800 \mathrm{~s} \times 0.54\right)=10.0$

The reaction quantum yield $(\Phi)$ was thus determined to be 10.0 . 


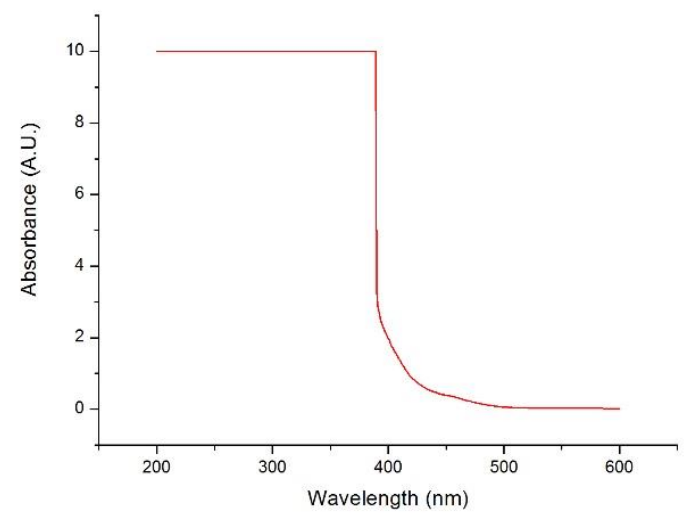

Figure S5. Absorbance of the reaction mixture solution.

\subsection{DFT Calculation on the Triplet Energies of intermediate 4a:}

A DFT calculation of the triplet energy of intermediate $\mathbf{4 a}$ was conducted and the obtained $\mathrm{ET}^{4 \mathrm{a}}(\mathrm{calc})=100.1 \mathrm{~kJ} / \mathrm{mol}$ is within the range that should be accessed using $\left[\operatorname{Ir}\left(\mathrm{dF}\left(\mathrm{CF}_{3}\right) \text { ppy }\right)_{2}\left(4,4^{\prime}-\mathrm{dCF}_{3}\right.\right.$ bpy $\left.)\right]\left(\mathrm{PF}_{6}\right) \quad$ as a triplet sensitizer $\quad\left(\mathrm{E}_{\mathrm{T}}^{\mathrm{PCIII}}=205.3\right.$ $\mathrm{kJ} / \mathrm{mol}){ }^{[18]}$ These results supported the possibility of energy transfer between the sensitizer and the substrate.

\section{Computational Details}

All of the calculations were performed with the Gaussian 16 B01 package. ${ }^{[19]}$ Geometry optimizations were carried out using M06-2X functional with basis set of 6-31+G(d,p). Vibrational frequencies were computed analytically at the same level of theory to confirm whether the structures are minimal. Solvation effects $($ solvent $=$ acetonitrile) were taken into account by performing single-point calculations with the SMD model.

$\mathrm{E}_{\mathrm{T}}{ }^{4 \mathrm{a}}($ calc $)=100.1 \mathrm{~kJ} / \mathrm{mol}$

\section{Energies and Cartesian coordinates of 4 a}




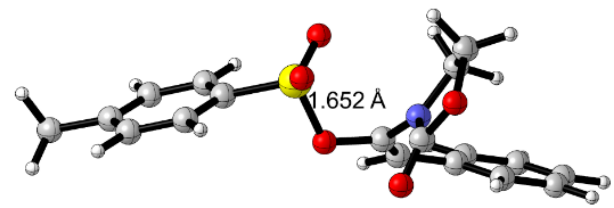

Singlet state

SCF Done: $\quad E(R M 062 X)=-1524.74875149$

Coordinate:

$\begin{array}{lrrr}\mathrm{C} & -1.42875100 & 2.21824900 & -0.85031700 \\ \mathrm{O} & -1.93800700 & 3.28100900 & -0.19978700 \\ \mathrm{C} & -2.15632100 & 2.94486700 & 1.18989400 \\ \mathrm{C} & -2.23324400 & 1.41638800 & 1.20657800 \\ \mathrm{H} & -1.30382600 & 3.31639700 & 1.76367200 \\ \mathrm{H} & -3.07566900 & 3.42909800 & 1.51360700 \\ \mathrm{H} & -1.73424500 & 0.97918400 & 2.07233700 \\ \mathrm{H} & -3.26317400 & 1.05224300 & 1.13955100 \\ \mathrm{O} & -1.02041400 & 2.25421600 & -1.98722800 \\ \mathrm{~N} & -1.49076700 & 1.11983100 & -0.01931400 \\ \mathrm{C} & -0.89126500 & -0.10028200 & -0.32811400 \\ \mathrm{C} & -1.46842500 & -1.30516700 & -0.34630300 \\ \mathrm{H} & -0.79833100 & -2.15889400 & -0.40610900 \\ \mathrm{C} & -2.91135200 & -1.58654300 & -0.28510500 \\ \mathrm{C} & -3.88539000 & -0.69456200 & -0.76213700 \\ \mathrm{C} & -3.32623400 & -2.82198100 & 0.23561700 \\ \mathrm{C} & -5.23704000 & -1.02371400 & -0.69400800 \\ \mathrm{H} & -3.58659800 & 0.25058800 & -1.20713400 \\ \mathrm{C} & -4.67900000 & -3.14625600 & 0.30888500 \\ \mathrm{H} & -2.57785800 & -3.52730900 & 0.58767200 \\ \mathrm{C} & -5.63988200 & -2.24591600 & -0.15268400 \\ \mathrm{H} & -5.97759500 & -0.32541500 & -1.07242500 \\ \mathrm{H} & -4.98188100 & -4.10391600 & 0.72155600 \\ \mathrm{H} & -6.69470600 & -2.49818100 & -0.10064200 \\ \mathrm{O} & 0.47786000 & -0.01565400 & -0.58333500 \\ \mathrm{~S} & 1.37786500 & 0.73068400 & 0.58351000 \\ \mathrm{O} & 0.85477000 & 0.29357500 & 1.86926500 \\ \mathrm{O} & 1.42376500 & 2.14960600 & 0.27534000 \\ \mathrm{C} & 2.93435800 & -0.01704300 & 0.24741300\end{array}$




$\begin{array}{lrrr}\mathrm{C} & 3.23009100 & -1.24295600 & 0.84103500 \\ \mathrm{C} & 3.82058500 & 0.62496500 & -0.61510100 \\ \mathrm{C} & 4.45634000 & -1.83412900 & 0.55905100 \\ \mathrm{H} & 2.52032300 & -1.72098300 & 1.50916900 \\ \mathrm{C} & 5.04048500 & 0.01288100 & -0.88260700 \\ \mathrm{H} & 3.56333500 & 1.57953500 & -1.06294800 \\ \mathrm{C} & 5.37552300 & -1.21699000 & -0.30067300 \\ \mathrm{H} & 4.70567000 & -2.78855800 & 1.01387500 \\ \mathrm{H} & 5.74471300 & 0.49753300 & -1.55295700 \\ \mathrm{C} & 6.71272700 & -1.84856500 & -0.57274800 \\ \mathrm{H} & 7.46605000 & -1.44272000 & 0.11174700 \\ \mathrm{H} & 6.67513200 & -2.93038200 & -0.42544500 \\ \mathrm{H} & 7.04517400 & -1.63948600 & -1.59259800\end{array}$

Triplet state

SCF Done: $\quad E(U M 062 X)=-1524.71061281$

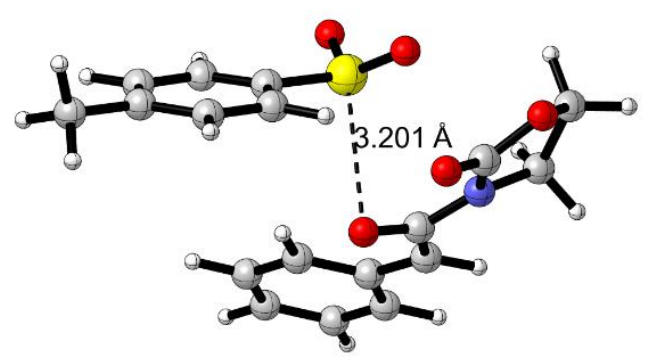

Coordinate

$\begin{array}{lrrr}\mathrm{C} & 1.29022300 & -2.79798800 & -0.94867500 \\ \mathrm{O} & 1.84878200 & -3.80868900 & -0.25860300 \\ \mathrm{C} & 2.80286800 & -3.30714700 & 0.69526600 \\ \mathrm{C} & 3.12841000 & -1.89175500 & 0.21168700 \\ \mathrm{H} & 2.32771200 & -3.30534000 & 1.67783600 \\ \mathrm{H} & 3.66724800 & -3.96902100 & 0.69025000 \\ \mathrm{H} & 3.15088900 & -1.18153600 & 1.04003600 \\ \mathrm{H} & 4.06960600 & -1.84641200 & -0.34237100 \\ \mathrm{O} & 0.36013700 & -2.95922400 & -1.69829400 \\ \mathrm{~N} & 1.99388700 & -1.62580200 & -0.67804200 \\ \mathrm{C} & 1.52273700 & -0.35062300 & -1.00735700 \\ \mathrm{C} & 2.43385000 & 0.74257700 & -0.69038300 \\ \mathrm{H} & 3.48854600 & 0.51220300 & -0.57983800 \\ \mathrm{C} & 2.03856300 & 2.10140600 & -0.51412800 \\ \mathrm{C} & 0.68934900 & 2.54559600 & -0.49637900\end{array}$




$\begin{array}{lrrr}\mathrm{C} & 3.06052600 & 3.06179000 & -0.29370800 \\ \mathrm{C} & 0.39547100 & 3.88300800 & -0.27200600 \\ \mathrm{H} & -0.11248600 & 1.83654400 & -0.66544600 \\ \mathrm{C} & 2.75387800 & 4.39610100 & -0.07778400 \\ \mathrm{H} & 4.09634400 & 2.73325300 & -0.29771400 \\ \mathrm{C} & 1.41877300 & 4.81456300 & -0.06496700 \\ \mathrm{H} & -0.64108200 & 4.20618800 & -0.25734700 \\ \mathrm{H} & 3.55145500 & 5.11452100 & 0.08432600 \\ \mathrm{H} & 1.17718000 & 5.85885300 & 0.10737300 \\ \mathrm{O} & 0.41318400 & -0.19162700 & -1.50811700 \\ \mathrm{~S} & -0.40559200 & -0.53612200 & 1.56318500 \\ \mathrm{O} & -0.44659000 & 0.10923600 & 2.89295300 \\ \mathrm{O} & -0.04175200 & -1.96649600 & 1.44812600 \\ \mathrm{C} & -1.97651200 & -0.27711600 & 0.75243500 \\ \mathrm{C} & -2.80205400 & 0.75050300 & 1.19891700 \\ \mathrm{C} & -2.28831600 & -1.07082800 & -0.35099100 \\ \mathrm{C} & -4.00989900 & 0.95478100 & 0.53582500 \\ \mathrm{H} & -2.51891900 & 1.36404000 & 2.04819900 \\ \mathrm{C} & -3.50122100 & -0.84643800 & -0.99063300 \\ \mathrm{H} & -1.59437900 & -1.82919900 & -0.70314100 \\ \mathrm{C} & -4.37650600 & 0.16177900 & -0.55815300 \\ \mathrm{H} & -4.67682200 & 1.74202700 & 0.87570700 \\ \mathrm{H} & -3.77258700 & -1.45834400 & -1.84682800 \\ \mathrm{C} & -5.69001200 & 0.37328000 & -1.25951600 \\ \mathrm{H} & -6.38507700 & -0.43760300 & -1.01577300 \\ \mathrm{H} & -6.15016800 & 1.31741500 & -0.96014300 \\ \mathrm{H} & -5.55605100 & 0.37445200 & -2.34501300\end{array}$

Triplet state energy determined from emission spectra of $\left[\operatorname{Ir}\left(\operatorname{dF}\left(C_{3}\right) p p y\right)_{2}\left(4,4^{\prime}-\right.\right.$ $\left.\left.\left(\mathrm{dCF}_{3}\right) \mathrm{bpy}\right)\right]\left(\mathrm{PF}_{6}\right)$

According to the following equation, triplet state energy of $\left[\operatorname{Ir}\left(\mathrm{dF}\left(\mathrm{CF}_{3}\right) \mathrm{ppy}\right)_{2}(4,4\right.$ '$\left(\mathrm{dCF}_{3}\right)$ bpy) $]\left(\mathrm{PF}_{6}\right)$ was calculated as below.

$\mathrm{E}=\mathrm{N}_{\mathrm{A}} \mathrm{hc} / \lambda=6.022 \times 10^{23} \times 6.626 \times 10^{-34} \times 3 \times 10^{8} /\left(583 \times 10^{-9}\right)=205.3 \mathrm{~kJ} / \mathrm{mol}$ 


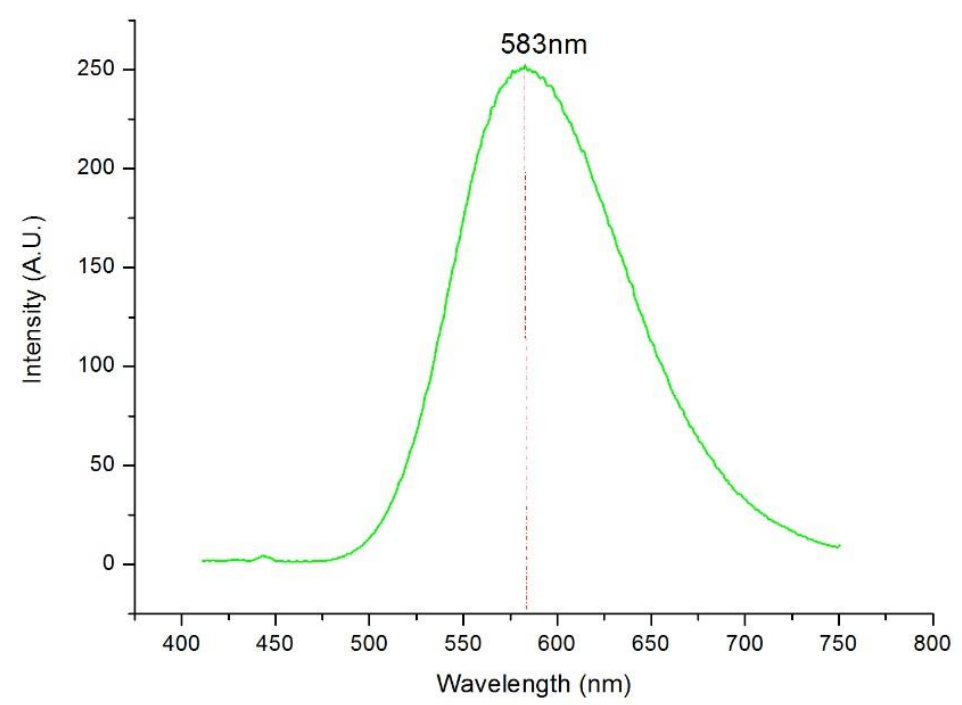

Figure S6. Emission spectra of $\left[\operatorname{Ir}\left(\mathrm{dF}_{\left(\mathrm{CF}_{3}\right)}\right) \text { ppy }\right)_{2}\left(4,4^{\prime}-\mathrm{dCF}_{3}\right.$ bpy) $]\left(\mathrm{PF}_{6}\right)$ (excitation wavelength, concentration in $\mathrm{MeCN})(392 \mathrm{~nm} 0.01 \mathrm{Mm})$ 


\section{Characterization of structurally novel compounds}

propyl phenyl(phenylethynyl)carbamate

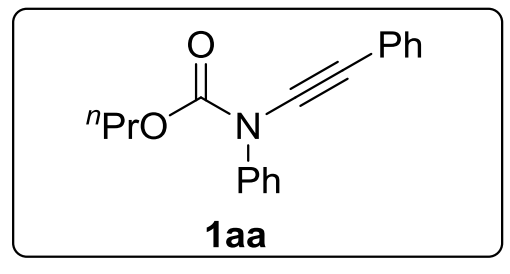

Following the general procedure, 1aa was obtained as a colorless oil $(0.70 \mathrm{~g}, 2.5 \mathrm{mmol}$, yield: $50 \%) .{ }^{1} \mathbf{H}$ NMR (401 MHz, CDCl $) \delta 7.57(\mathrm{~d}, J=8.0 \mathrm{~Hz}, 2 \mathrm{H})$, $7.42(\mathrm{~m}, 4 \mathrm{H}), 7.30(\mathrm{~m}, 4 \mathrm{H}), 4.26(\mathrm{t}, J=6.2 \mathrm{~Hz}, 2 \mathrm{H})$, $1.77(\mathrm{~h}, J=6.7 \mathrm{~Hz}, 2 \mathrm{H}), 1.02(\mathrm{t}, J=7.2 \mathrm{~Hz}, 3 \mathrm{H}) . ;{ }^{13} \mathbf{C}$ NMR $\left(401 \mathbf{M H z}, \mathbf{C D C l}_{3}\right) \delta$ $154.3,139.5,131.1,128.9,128.2,127.6,126.8,124.5,123.0,82.9,70.1,69.1$, $22.0,10.3$. HRMS (ESI, m/z): calculated for $\mathrm{C}_{18} \mathrm{H}_{17} \mathrm{NO}_{2}[\mathrm{M}+\mathrm{H}]^{+}: 280.1338$, found: 280.1338 .

\section{propyl (4-phenoxyphenyl)(phenylethynyl)carbamate}

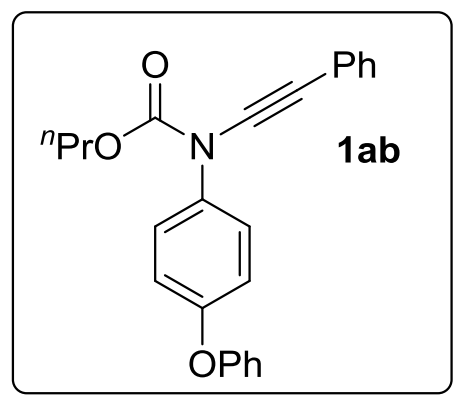

Following the general procedure, $\mathbf{1}$ ab was obtained as a colorless oil (1.17 g, 3.15 mmol, yield: 63\%).. ${ }^{\mathbf{1}} \mathbf{H}$ NMR (401 MHz, $\left.\mathbf{C D C l}_{3}\right) \delta 7.53-7.42(\mathrm{~m}, 2 \mathrm{H}), 7.43-7.38(\mathrm{~m}$, 2H), $7.37-7.32(\mathrm{~m}, 2 \mathrm{H}), 7.32-7.26(\mathrm{~m}, 3 \mathrm{H}), 7.15-7.09$ $(\mathrm{m}, 1 \mathrm{H}), 7.06-6.99(\mathrm{~m}, 4 \mathrm{H}), 4.24(\mathrm{t}, J=6.6 \mathrm{~Hz}, 2 \mathrm{H})$, $1.76(\mathrm{~h}, 2 \mathrm{H}), 1.01(\mathrm{t}, J=7.4 \mathrm{~Hz}, 3 \mathrm{H}) . ;{ }^{13} \mathbf{C}$ NMR (401 MHz, $\left.\mathbf{C D C l}_{3}\right) \delta 156.7,156.0,154.5,134.5,131.1,129.8,128.2,127.6,126.2$, $123.6,123.0,119.2,118.8,83.0,70.0,69.1,22.1,10.3$. HRMS (ESI, m/z): calculated for $\mathrm{C}_{24} \mathrm{H}_{21} \mathrm{NO}_{3}[\mathrm{M}+\mathrm{H}]^{+}: 372.1600$, found: 372.1602 .

\section{3-(2-phenyl-2-tosylacetyl)oxazolidin-2-one}

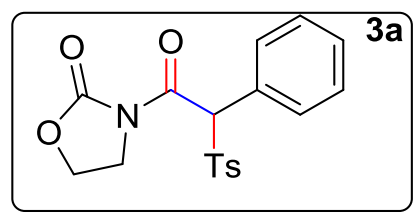

Following the general procedure, 3a was obtained as a colorless oil (28.0 mg, $0.078 \mathrm{mmol}$, yield: 78\%). ${ }^{1} \mathbf{H}$ NMR (400 MHz, $\left.\mathbf{C D C l}_{3}\right): \delta 7.36(\mathrm{~d}, J=8.3 \mathrm{~Hz}, 2 \mathrm{H}), 7.31-7.26$ (m, 3H), $7.22-7.17(\mathrm{~m}, 2 \mathrm{H}), 7.13(\mathrm{~d}, J=8.1 \mathrm{~Hz}, 2 \mathrm{H}), 6.86$ (s, 1H), $4.43-4.19$ (m, 2H), $4.13-3.99$ (m, 1H), 3.95 - 3.78 (m, 1H), 2.33 (s, 3H); ${ }^{13}$ C NMR (101 MHz, $\left.\mathbf{C D C l}_{3}\right): \delta 164.1,153.0,145.2,133.7,130.9,129.8,129.6,129.2,128.5,127.7$, 71.2, $62.0,42.8,21.7$. HRMS (ESI, m/z): calculated for $\mathrm{C}_{18} \mathrm{H}_{17} \mathrm{NO}_{5} \mathrm{~S}[\mathrm{M}+\mathrm{H}]^{+}$: 
360.0906, found: 360.0906 .

3-(2-(4-methoxyphenyl)-2-tosylacetyl)oxazolidin-2-one

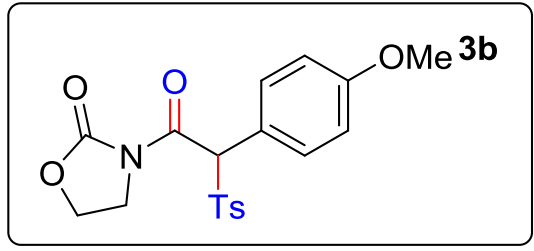

Following the general procedure, $\mathbf{3 b}$ was obtained as a colorless oil (32.3 mg, $0.083 \mathrm{mmol}$, yield: 83\%). ${ }^{1}$ H NMR (400 MHz, $\left.\mathbf{C D C l}_{3}\right): \delta 7.40(\mathrm{~d}, \mathrm{~J}=8.2 \mathrm{~Hz}$, 2H), $7.21(\mathrm{~d}, J=8.9 \mathrm{~Hz}, 2 \mathrm{H}), 7.15(\mathrm{~d}, J=8.1 \mathrm{~Hz}$, 2H), $6.73(\mathrm{~s}, 1 \mathrm{H}), 6.71(\mathrm{~d}, J=1.8 \mathrm{~Hz}, 2 \mathrm{H}), 4.39-4.25(\mathrm{~m}, 2 \mathrm{H}), 4.11-4.03(\mathrm{~m}, 1 \mathrm{H})$, 3.95 - $3.86(\mathrm{~m}, 1 \mathrm{H}), 3.72(\mathrm{~s}, 3 \mathrm{H}), 2.34$ (s, 3H); ${ }^{13} \mathbf{C}$ NMR (101 MHz, CDCl $)$ ): $\delta 164.4$, $160.7,153.0$, 145.2 , 133.9 , 132.3 , 129.9 , 129.2 , 119.6 , 113.9 , 70.7 , 62.0 , 55.3 , 42.8 , 21.7 . HRMS (ESI, m/z): calculated for $\mathrm{C}_{19} \mathrm{H}_{19} \mathrm{NO}_{6} \mathrm{~S}[\mathrm{M}+\mathrm{H}]^{+}: 390.1011$, found: 390.1013.

\section{3-(2-(2-methoxyphenyl)-2-tosylacetyl)oxazolidin-2-one}

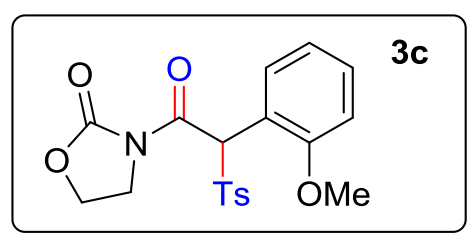

Following the general procedure, $\mathbf{3 c}$ was obtained as a colorless oil (27.6 mg, $0.071 \mathrm{mmol}$, yield: $71 \%)$. ${ }^{1} \mathbf{H}$ NMR (400 MHz, $\left.\mathbf{C D C l}_{3}\right): \delta 7.90(\mathrm{dd}, J=7.8,1.7 \mathrm{~Hz}$, 1H), $7.68(\mathrm{~s}, 1 \mathrm{H}), 7.51(\mathrm{~d}, J=8.3 \mathrm{~Hz}, 2 \mathrm{H}), 7.30(\mathrm{td}, J=$ 8.3, $1.7 \mathrm{~Hz}, 1 \mathrm{H}), 7.19(\mathrm{~d}, J=8.0 \mathrm{~Hz}, 2 \mathrm{H}), 6.96(\mathrm{td}, J=7.7,0.9 \mathrm{~Hz}, 1 \mathrm{H}), 6.68(\mathrm{~d}, J=$ $8.3 \mathrm{~Hz}, 1 \mathrm{H}), 4.45-4.35(\mathrm{~m}, 2 \mathrm{H}), 4.23-4.14(\mathrm{~m}, 1 \mathrm{H}), 4.04-3.96(\mathrm{~m}, 1 \mathrm{H}), 3.41(\mathrm{~s}$, 3H), 2.39 (s, 3H); ${ }^{13} \mathbf{C}$ NMR (101 MHz, CDCl 3 ): $\delta 163.9,157.6,152.9,144.8,135.0$, 131.9 , 130.9 , $129.6,129.0,120.6,116.3,110.5$, 62.4 , 61.9 , 55.5, 43.1 , 21.7.

HRMS (ESI, m/z): calculated for $\mathrm{C}_{19} \mathrm{H}_{19} \mathrm{NO}_{6} \mathrm{~S}[\mathrm{M}+\mathrm{H}]^{+}: 390.1011$, found: 390.1011 .

\section{3-(2-(4-(tert-butyl)phenyl)-2-tosylacetyl)oxazolidin-2-one}

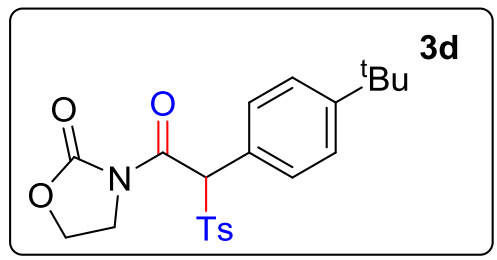

Following the general procedure, $\mathbf{3 d}$ was obtained as a colorless oil (30 mg, $0.072 \mathrm{mmol}$, yield: $72 \%) .{ }^{1} \mathbf{H}$ NMR (400 MHz, CDCl $): \delta 7.46(\mathrm{~d}, J=8.3 \mathrm{~Hz}, 2 \mathrm{H})$, 
$7.29(\mathrm{~s}, 4 \mathrm{H}), 7.20(\mathrm{~d}, J=8.1 \mathrm{~Hz}, 1 \mathrm{H}), 6.83(\mathrm{~s}, 2 \mathrm{H}), 4.37(\mathrm{~m}, J=22.1,9.0,7.0 \mathrm{~Hz}, 1 \mathrm{H})$, $4.11(\mathrm{ddd}, J=11.0,9.3,6.4 \mathrm{~Hz}, 2 \mathrm{H}), 3.96(\mathrm{ddd}, J=11.0,9.3,7.4 \mathrm{~Hz}, 1 \mathrm{H}), 2.41(\mathrm{~s}, 3 \mathrm{H})$, $1.28(\mathrm{~s}, 9 \mathrm{H}) ;{ }^{13} \mathbf{C}$ NMR (101 MHz, $\left.\mathbf{C D C l}_{3}\right): \delta 164.3,153.0,145.1,134.0,130.6$, $129.9,129.2,125.5,124.6,71.0,62.0,42.8,34.7,31.2$, 21.7 . HRMS (ESI, m/z): calculated for $\mathrm{C}_{22} \mathrm{H}_{25} \mathrm{NO}_{5} \mathrm{~S}[\mathrm{M}+\mathrm{H}]^{+}$: 416.5132, found:416.5138.

\section{3-(2-(o-tolyl)-2-tosylacetyl)oxazolidin-2-one}

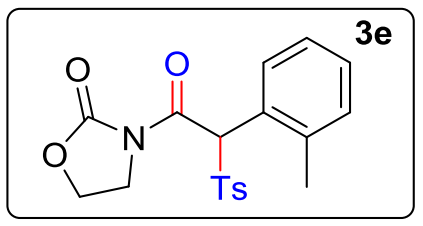

Following the general procedure, $\mathbf{3 e}$ was obtained as a colorless oil (28.4 mg, 0.076 mmol, yield: $76 \%)$. ${ }^{1}$ H NMR (400 MHz, $\left.\mathbf{C D C l}_{3}\right): \delta 7.60(\mathrm{~d}, J=7.8 \mathrm{~Hz}, 1 \mathrm{H}), 7.49(\mathrm{~d}, J=$ $8.2 \mathrm{~Hz}, 2 \mathrm{H}), 7.32-7.20(\mathrm{~m}, 4 \mathrm{H}), 7.14(\mathrm{t}, J=7.6 \mathrm{~Hz}, 2 \mathrm{H})$, $4.50-4.32(\mathrm{~m}, 2 \mathrm{H}), 4.17(\mathrm{~m}, J=10.1,9.3,6.6 \mathrm{~Hz}, 1 \mathrm{H}), 4.05-3.93(\mathrm{~m}, 1 \mathrm{H}), 2.43(\mathrm{~s}$, 3H), 2.21 (s, 3H); ${ }^{13} \mathbf{C}$ NMR (101 MHz, CDCl 3 ): $\delta$ 164.4 , 153.1 , 145.3 , 139.7 , 134.2 , $130.8,130.4,130.0,129.6,129.2,126.1,125.9$, 67.0 , 62.0 , 43.0 , $21.7,19.4$. HRMS (ESI, m/z): calculated for $\mathrm{C}_{19} \mathrm{H}_{19} \mathrm{NO}_{5} \mathrm{~S}[\mathrm{M}+\mathrm{H}]^{+}: 374.1062$, found: 374.1060 .

\section{3-(2-(m-tolyl)-2-tosylacetyl)oxazolidin-2-one}

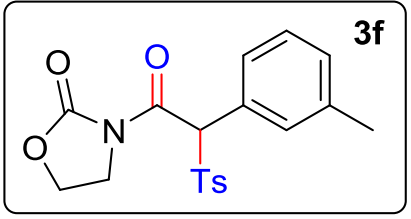

Following the general procedure, $\mathbf{3 f}$ was obtained as a colorless oil (29.9 mg, $0.080 \mathrm{mmol}$, yield: 80\%). ${ }^{\mathbf{1}} \mathbf{H}$ NMR (400 MHz, $\left.\mathbf{C D C l}_{3}\right): \delta 7.46(\mathrm{~d}, J=8.3 \mathrm{~Hz}, 2 \mathrm{H}), 7.21(\mathrm{~d}, J=$

$8.1 \mathrm{~Hz}, 2 \mathrm{H}), 7.19-7.13(\mathrm{~m}, 3 \mathrm{H}), 7.11(\mathrm{t}, 1 \mathrm{H}), 6.81(\mathrm{~s}, 1 \mathrm{H}), 4.48-4.28(\mathrm{~m}, 2 \mathrm{H}), 4.19$ - 4.07 (m, 1H), 4.04 - 3.92 (m, 1H), 2.41 (s, 3H), 2.27 (s, 3H); ${ }^{13}$ C NMR (101 MHz, $\left.\mathbf{C D C l}_{3}\right): \delta 164.3,153.0,145.2,138.3,133.8,131.5,130.5,130.0,129.1,128.3$, $128.0,127.5,71.4,62.0,42.8,21.7,21.3$. HRMS (ESI, m/z): calculated for $\mathrm{C}_{19} \mathrm{H}_{19} \mathrm{NO}_{5} \mathrm{~S}[\mathrm{M}+\mathrm{H}]^{+}:$374.1062, found: 374.1061 . 


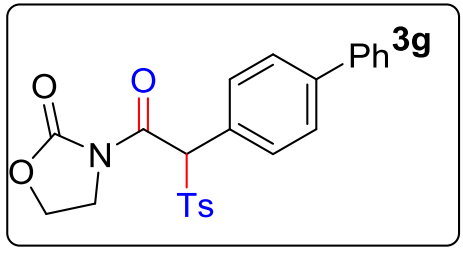

Following the general procedure, $\mathbf{3 g}$ was obtained as a white solid (30.5 mg, 0.07mmol, yield: $70 \%) .{ }^{1} \mathbf{H}$ NMR (400 MHz, $\left.\mathbf{C D C l}_{3}\right): \delta 7.57(\mathrm{~d}, J=7.3 \mathrm{~Hz}, 2 \mathrm{H}), 7.52(\mathrm{~d}$, $J=6.7 \mathrm{~Hz}, 4 \mathrm{H}), 7.47-7.41(\mathrm{~m}, 4 \mathrm{H}), 7.37(\mathrm{t}, J=7.3 \mathrm{~Hz}$,

1H), $7.22(\mathrm{~d}, J=8.1 \mathrm{~Hz}, 2 \mathrm{H}), 6.92(\mathrm{~s}, 1 \mathrm{H}), 4.49-4.33(\mathrm{~m}, 2 \mathrm{H}), 4.23-4.11(\mathrm{~m}, 1 \mathrm{H})$, $4.06-3.94(\mathrm{~m}, 1 \mathrm{H}), 2.41(\mathrm{~s}, 3 \mathrm{H}) ;{ }^{13} \mathbf{C}$ NMR (101 MHz, $\left.\mathbf{C D C l}_{3}\right): \delta 164.1,153.0$, $145.3,142.4,140.0,133.8,131.3,129.9$, 129.2 , $128.8,127.8,127.1$, 127.1 , 126.6 , $71.0,62.0,42.8,21.7$. HRMS (ESI, m/z): calculated for $\mathrm{C}_{24} \mathrm{H}_{21} \mathrm{NO}_{5} \mathrm{~S}[\mathrm{M}+\mathrm{H}]^{+}$: 436.1219, found: 436.1219 .

\section{3-(2-tosyl-2-(2-(trifluoromethoxy)phenyl)acetyl)oxazolidin-2-one}

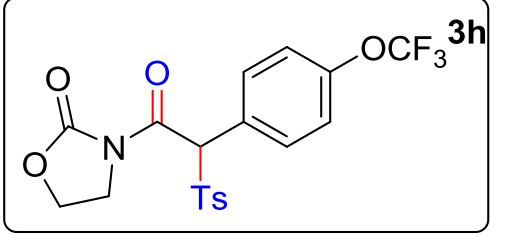

Following the general procedure, $\mathbf{3 h}$ was obtained as a colorless oil (35.5 mg, $0.08 \mathrm{mmol}$, yield: $80 \%$ ). ${ }^{1} \mathbf{H}$ NMR (400 MHz, $\left.\mathbf{C D C l}_{3}\right): \delta 7.46(\mathrm{dd}, J=11.8,8.6 \mathrm{~Hz}$, $4 \mathrm{H}), 7.23(\mathrm{~d}, J=8.1 \mathrm{~Hz}, 2 \mathrm{H}), 7.13(\mathrm{~d}, J=8.2 \mathrm{~Hz}, 2 \mathrm{H})$, $6.90(\mathrm{~s}, 1 \mathrm{H}), 4.52-4.34(\mathrm{~m}, 2 \mathrm{H}), 4.24-4.10(\mathrm{~m}, 1 \mathrm{H}), 4.08-3.94(\mathrm{~m}, 1 \mathrm{H}), 2.42(\mathrm{~s}$, 3H); ${ }^{13}$ C NMR (101 MHz, CDCl $): \delta 163.7,153.1,150.1,145.6,133.4,132.5$, $129.7,129.3,126.3,120.7,120.5$ (q, $J=257.9$ Hz) , 70.1 , 62.1 , 42.8 , 21.7 . HRMS (ESI, m/z): calculated for $\mathrm{C}_{19} \mathrm{H}_{16} \mathrm{~F}_{3} \mathrm{NO}_{6} \mathrm{~S}[\mathrm{M}+\mathrm{H}]^{+}:$444.0729, found: 444.0728 .

\section{3-(2-tosyl-2-(4-(trifluoromethyl)phenyl)acetyl)oxazolidin-2-one}

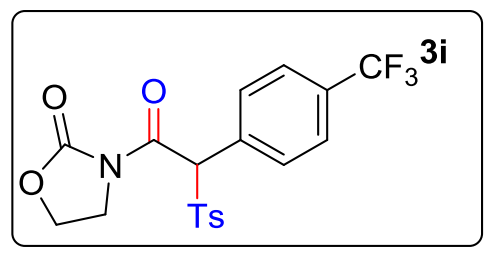

Following the general procedure, $\mathbf{3 i}$ was obtained as a white solid (22.6 mg, $0.053 \mathrm{mmol}$, yield: $53 \%)$. ${ }^{1} \mathbf{H}$ NMR (401 MHz, CDCl 3$): \delta 7.54(\mathrm{~s}, 4 \mathrm{H}), 7.48(\mathrm{~d}, J=$ $8.3 \mathrm{~Hz}, 2 \mathrm{H}), 7.24(\mathrm{~d}, J=8.0 \mathrm{~Hz}, 2 \mathrm{H}), 6.96(\mathrm{~s}, 1 \mathrm{H}), 4.48$ $-4.42(\mathrm{~m}, 1 \mathrm{H}), 4.40(\mathrm{~m}, J=9.1,3.0 \mathrm{~Hz}, 1 \mathrm{H}), 4.16(\mathrm{ddd}, J=11.1,9.2,6.1 \mathrm{~Hz}, 1 \mathrm{H})$, $4.01(\mathrm{ddd}, J=11.1,9.2,7.6 \mathrm{~Hz}, 1 \mathrm{H}), 2.43(\mathrm{~s}, 3 \mathrm{H}) ;{ }^{13} \mathbf{C}$ NMR $\left(\mathbf{1 0 1} \mathbf{M H z}, \mathbf{C D C l}_{3}\right) \delta$ $163.51,153.07,145.72,133.55,131.7$ (q, $J=32.7 \mathrm{~Hz}), 131.7,131.46,129.75$, $129.47,125.34(\mathrm{q}, J=3.7 \mathrm{~Hz}), 123.7(\mathrm{q}, J=273.7 \mathrm{~Hz}), 70.59,62.08,42.86,21.73$. 
HRMS (ESI, m/z): calculated for $\mathrm{C}_{19} \mathrm{H}_{16} \mathrm{~F}_{3} \mathrm{NO}_{5} \mathrm{~S}[\mathrm{M}+\mathrm{H}]^{+}: 428.0780$, found: 428.0780 .

\section{Methyl-4-(2-oxo-2-(2-oxooxazolidin-3-yl)-1-tosylethyl)benzoate}

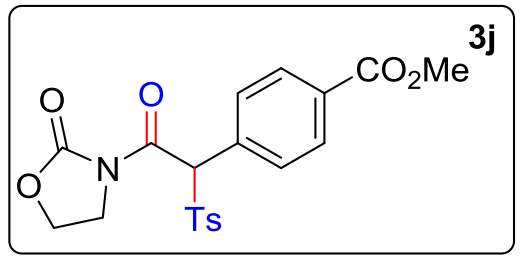

Following the general procedure, $\mathbf{3} \mathbf{j}$ was obtained as a white solid (18.8 mg, $0.045 \mathrm{mmol}$, yield: $45 \%) .{ }^{1} \mathbf{H}$ NMR (400 MHz, CDCl $)$ ): $\delta 7.94(\mathrm{~d}, J=8.3 \mathrm{~Hz}, 2 \mathrm{H})$, $7.46(\mathrm{dd}, J=8.0,5.9 \mathrm{~Hz}, 4 \mathrm{H}), 7.22(\mathrm{~d}, J=8.1 \mathrm{~Hz}, 2 \mathrm{H})$, $6.95(\mathrm{~s}, 1 \mathrm{H}), 4.52-4.35(\mathrm{~m}, 2 \mathrm{H}), 4.24-4.13(\mathrm{~m}, 1 \mathrm{H}), 4.08-3.98(\mathrm{~m}, 1 \mathrm{H}), 3.92(\mathrm{~s}$, 3H), 2.42 (s, 3H); ${ }^{13}$ C NMR (101 MHz, CDCl $)$ ): $\delta$ 166.5 , 163.6 , 153.0 , 145.6, 133.5 , $132.5,131.2,131.02,129.8,129.5,129.4,70.9,62.0,52.3,42.8,21.7$. HRMS

(ESI, m/z): calculated for $\mathrm{C}_{20} \mathrm{H}_{19} \mathrm{NO}_{7} \mathrm{~S}[\mathrm{M}+\mathrm{H}]^{+}:$: 418.0960, found: 418.0960 .

\section{3-(2-(4-fluorophenyl)-2-tosylacetyl)oxazolidin-2-one}

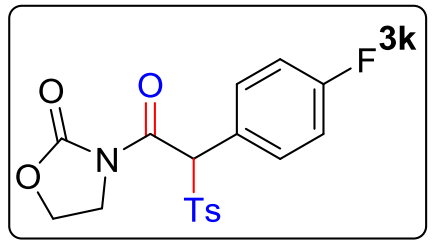

Following the general procedure, $\mathbf{3 k}$ was obtained as a colorless oil (27.2 mg, $0.072 \mathrm{mmol}$, yield: 72\%). ${ }^{1} \mathbf{H}$ NMR (400 MHz, $\left.\mathbf{C D C l}_{3}\right): \delta 7.45(\mathrm{~d}, J=8.2 \mathrm{~Hz}, 2 \mathrm{H}), 7.36(\mathrm{dd}, J$ $=8.8,5.3 \mathrm{~Hz}, 2 \mathrm{H}), 7.22(\mathrm{~d}, J=8.1 \mathrm{~Hz}, 2 \mathrm{H}), 6.96(\mathrm{t}, J=8.6$ $\mathrm{Hz}, 2 \mathrm{H}), 6.85$ (s, 1H), $4.49-4.33(\mathrm{~m}, 2 \mathrm{H}), 4.20-4.09$ (m, 1H), $4.03-3.95(\mathrm{~m}, 1 \mathrm{H})$, $2.41(\mathrm{~s}, 3 \mathrm{H}) ;{ }^{13} \mathbf{C} \mathbf{N M R}\left(\mathbf{1 0 1} \mathbf{M H z}, \mathbf{C D C l}_{3}\right): \delta 164.0, \delta 163.6(\mathrm{~d}, J=250.1 \mathrm{~Hz}), 153.1$, $145.5,133.6, \delta 132.9(\mathrm{~d}, J=8.6 \mathrm{~Hz}), 129.8,129.4, \delta 123.7(\mathrm{~d}, J=3.2 \mathrm{~Hz}), \delta 115.6$ $(\mathrm{d}, J=21.7 \mathrm{~Hz}), 70.2,62.1,42.8,21.7$. HRMS (ESI, m/z): calculated for $\mathrm{C}_{18} \mathrm{H}_{16} \mathrm{FNO}_{5} \mathrm{~S}[\mathrm{M}+\mathrm{H}]^{+}: 378.0811$, found: 378.0805 .

\section{3-(2-(3-fluorophenyl)-2-tosylacetyl)oxazolidin-2-one}

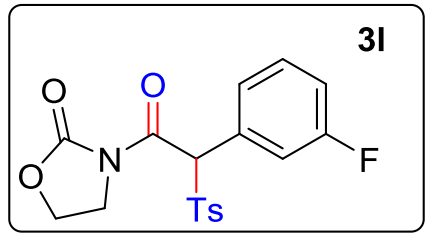

Following the general procedure, $\mathbf{3 1}$ was obtained as a colorless oil (23.8 mg, 0.063 mmol, yield: 63\%). ${ }^{1} \mathbf{H}$ NMR $\left(400 \mathrm{MHz}, \mathbf{C D C l}_{3}\right): \delta 7.49(\mathrm{~d}, J=8.2 \mathrm{~Hz}, 2 \mathrm{H}), 7.24(\mathrm{~d}, J$ $=8.1 \mathrm{~Hz}, 3 \mathrm{H}), 7.19(\mathrm{~d}, J=9.7 \mathrm{~Hz}, 1 \mathrm{H}), 7.13(\mathrm{~d}, J=7.8 \mathrm{~Hz}$ 1H), $7.07(\mathrm{td}, J=8.3,2.4 \mathrm{~Hz}, 1 \mathrm{H}), 6.89(\mathrm{~s}, 1 \mathrm{H}), 4.49-4.42(\mathrm{~m}, 1 \mathrm{H}), 4.42-4.36(\mathrm{~m}$, 1H), $4.22-4.12(\mathrm{~m}, 1 \mathrm{H}), 4.06-3.97(\mathrm{~m}, 1 \mathrm{H}), 2.43(\mathrm{~s}, 3 \mathrm{H}) ;{ }^{13} \mathbf{C}$ NMR (101 MHz, 
CDCl3) $\delta 163.67,162.33(\mathrm{~d}, \mathrm{~J}=247.3 \mathrm{~Hz}), 153.02,145.53,133.53,129.9(\mathrm{~d}, \mathrm{~J}=8.3$ Hz), 129.8 (d, J = 8.1 Hz), 129.77 , 129.33 , 126.88 (d, J = 3.1 Hz), 117.90 (d, J = 23.3 Hz), $116.75(\mathrm{~d}, \mathrm{~J}=21.0 \mathrm{~Hz}), 70.50,62.02,42.82,21.71$. HRMS (ESI, m/z): calculated for $\mathrm{C}_{18} \mathrm{H}_{16} \mathrm{FNO}_{5} \mathrm{~S}[\mathrm{M}+\mathrm{H}]^{+}: 378.0811$, found: 378.0804 .

\section{3-(2-(2-fluorophenyl)-2-tosylacetyl)oxazolidin-2-one}

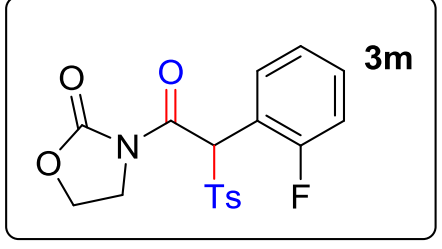

Following the general procedure, $\mathbf{3 m}$ was obtained as a colorless oil (20.0 mg, $0.053 \mathrm{mmol}$, yield: 53\%). ${ }^{1} \mathbf{H}$ NMR (400 MHz, CDCl 3$): \delta 7.92(\mathrm{t}, J=7.5 \mathrm{~Hz}, 1 \mathrm{H}), 7.53(\mathrm{~d}, J$ $=8.2 \mathrm{~Hz}, 2 \mathrm{H}), 7.42(\mathrm{~s}, 1 \mathrm{H}), 7.38-7.31(\mathrm{~m}, 1 \mathrm{H}), 7.23(\mathrm{~d}$, $J=8.1 \mathrm{~Hz}, 2 \mathrm{H}), 7.16(\mathrm{t}, J=7.5 \mathrm{~Hz}, 1 \mathrm{H}), 6.92(\mathrm{t}, J=9.0 \mathrm{~Hz}, 1 \mathrm{H}), 4.50-4.43(\mathrm{~m}, 1 \mathrm{H})$, $4.43-4.35(\mathrm{~m}, 1 \mathrm{H}), 4.25-4.15(\mathrm{~m}, 1 \mathrm{H}), 4.08-3.98(\mathrm{~m}, 1 \mathrm{H}), 2.42(\mathrm{~s}, 3 \mathrm{H}) ;{ }^{13} \mathbf{C}$ NMR $\left(\mathbf{1 0 1} \mathbf{M H z}, \mathbf{C D C l}_{3}\right) \delta 163.2,160.9(\mathrm{~d}, J=251.9 \mathrm{~Hz}), 152.9,145.5,134.1,132.2$, 132.1, $131.5(\mathrm{~d}, J=8.5 \mathrm{~Hz}), 129.4,124.2(\mathrm{~d}, J=3.7 \mathrm{~Hz}), 115.5(\mathrm{~d}, J=13.0 \mathrm{~Hz}), 115.2$ (d, $J=22.2 \mathrm{~Hz}), 62.4$ (d, $J=2.8 \mathrm{~Hz}), 62.0,43.0,21.7$. HRMS (ESI, m/z): calculated for $\mathrm{C}_{18} \mathrm{H}_{16} \mathrm{FNO}_{5} \mathrm{~S}[\mathrm{M}+\mathrm{H}]^{+}: 378.0811$, found: 378.0805 .

\section{3-(2-(4-chlorophenyl)-2-tosylacetyl)oxazolidin-2-one}

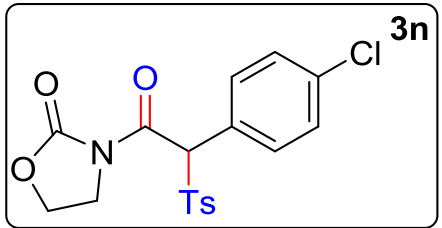

Following the general procedure, 3n was obtained as a colorless oil (28.3 mg, $0.072 \mathrm{mmol}$, yield: $72 \%) .{ }^{1} \mathbf{H}$ NMR (400 MHz, $\left.\mathbf{C D C l}_{3}\right): \delta 7.47(\mathrm{~d}, J=8.1 \mathrm{~Hz}, 2 \mathrm{H}), 7.32(\mathrm{~d}, J$ $=8.5 \mathrm{~Hz}, 2 \mathrm{H}), 7.28-7.21(\mathrm{~m}, 4 \mathrm{H}), 6.85(\mathrm{~s}, 1 \mathrm{H}), 4.48-$ $4.35(\mathrm{~m}, 2 \mathrm{H}), 4.19-4.11(\mathrm{~m}, 1 \mathrm{H}), 4.05-3.96(\mathrm{~m}, 1 \mathrm{H}), 2.42(\mathrm{~s}, 3 \mathrm{H}) ;{ }^{13} \mathbf{C}$ NMR (101 MHz, $\left.\mathbf{C D C l}_{3}\right): \delta 163.8,153.1,145.5,136.0,133.6,132.3,129.8,129.4,128.7$, $126.3,70.4,62.1,42.9,21.8$. HRMS (ESI, m/z): calculated for $\mathrm{C}_{18} \mathrm{H}_{16} \mathrm{ClNO}_{5} \mathrm{~S}$ $[\mathrm{M}+\mathrm{H}]^{+}:$394.0516, found: 394.0517 . 
3-(2-(3-bromophenyl)-2-tosylacetyl)oxazolidin-2-one

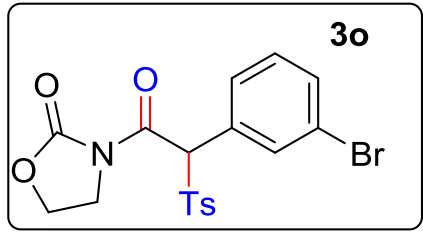

Following the general procedure, 3o was obtained as a colorless oil (26.2 mg, 0.06mmol, yield: $60 \%) .{ }^{1} \mathbf{H}$ NMR (400 MHz, $\left.\mathbf{C D C l}_{3}\right): \delta 7.53-7.45(\mathrm{~m}, 4 \mathrm{H}), 7.34(\mathrm{~d}, J=7.9$ $\mathrm{Hz}, 1 \mathrm{H}), 7.25(\mathrm{~d}, J=8.5 \mathrm{~Hz}, 2 \mathrm{H}), 7.16(\mathrm{t}, J=7.9 \mathrm{~Hz}, 1 \mathrm{H})$, $6.84(\mathrm{~s}, 1 \mathrm{H}), 4.48-4.38(\mathrm{~m}, 2 \mathrm{H}), 4.21-4.10(\mathrm{~m}, 1 \mathrm{H}), 4.06-3.97(\mathrm{~m}, 1 \mathrm{H}), 2.44(\mathrm{~s}$, 3H).; ${ }^{13} \mathbf{C}$ NMR (101 MHz, $\left.\mathbf{C D C l}_{3}\right): \delta 163.8,153.1,145.8,133.7,133.4,132.9$, $130.0,130.0,129.9,129.5,122.4,70.7,62.2,42.9,21.8$. HRMS (ESI, m/z): calculated for $\mathrm{C}_{18} \mathrm{H}_{16} \mathrm{BrNO}_{5} \mathrm{~S}[\mathrm{M}+\mathrm{H}]^{+}:$438.0011, found: 438.0011 .

\section{3-(2-(2-bromo-4-methoxyphenyl)-2-tosylacetyl)oxazolidin-2-one}

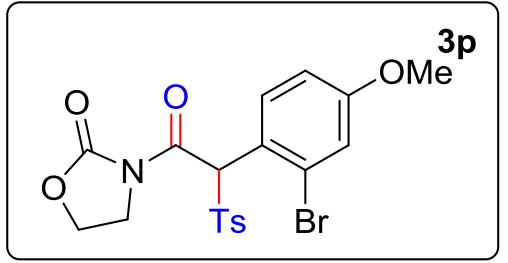

Following the general procedure, $\mathbf{3 p}$ was obtained as a colorless oil (42.0 mg, $0.042 \mathrm{mmol}$, yield: 90\%). ${ }^{1} \mathbf{H}$ NMR (401 MHz, CDCl $): \delta 8.15(\mathrm{~d}, J=8.9 \mathrm{~Hz}, 1 \mathrm{H})$, $7.71(\mathrm{~s}, 1 \mathrm{H}), 7.51(\mathrm{~d}, J=8.3 \mathrm{~Hz}, 2 \mathrm{H}), 7.22(\mathrm{~d}, J=8.3$ $\mathrm{Hz}, 2 \mathrm{H}), 6.94(\mathrm{~d}, J=2.7 \mathrm{~Hz}, 1 \mathrm{H}), 6.90(\mathrm{dd}, J=8.9,2.7$ $\mathrm{Hz}, 1 \mathrm{H}), 4.48-4.42(\mathrm{~m}, 1 \mathrm{H}), 4.41-4.36(\mathrm{~m}, 1 \mathrm{H}), 4.22-4.14(\mathrm{~m}, 1 \mathrm{H}), 4.06-3.98(\mathrm{~m}$, 1H), 3.77 (s, 3H), 2.40 (s, 3H); ${ }^{13}$ C NMR (101 MHz, CDCl 3$): \delta 163.2,160.7,153.0$, $145.4,134.5,134.2,129.5,129.4,127.1,119.5,117.8,113.3,67.3,61.9,55.5$, 43.1 , 21.7. HRMS (ESI, m/z): calculated for $\mathrm{C}_{19} \mathrm{H}_{18} \mathrm{BrNO}_{6} \mathrm{~S}[\mathrm{M}+\mathrm{H}]^{+}: 468.0116$, found: 468.0116 .

\section{3-(2-(3-chloro-4-methoxyphenyl)-2-tosylacetyl)oxazolidin-2-one}

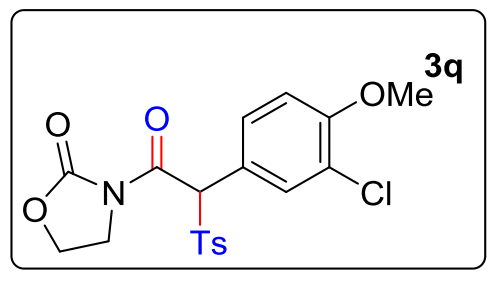

Following the general procedure, $\mathbf{3 q}$ was obtained as a white solid (31.7 mg, $0.075 \mathrm{mmol}$, yield: $75 \%) .{ }^{1} \mathbf{H}$ NMR (401 MHz, $\left.\mathbf{C D C l}_{3}\right) \delta 7.42(\mathrm{~d}, J=8.2 \mathrm{~Hz}, 2 \mathrm{H})$, $7.26-7.22(\mathrm{~m}, 2 \mathrm{H}), 7.18(\mathrm{~d}, J=8.2 \mathrm{~Hz}, 2 \mathrm{H}), 6.77(\mathrm{~d}, J$ $=9.2 \mathrm{~Hz}, 1 \mathrm{H}), 6.71(\mathrm{~s}, 1 \mathrm{H}), 4.44-4.22(\mathrm{~m}, 2 \mathrm{H}), 4.16-4.01(\mathrm{~m}, 1 \mathrm{H}), 3.97-3.89(\mathrm{~m}$, 1H), 3.82 (s, 3H), 2.36 (s, 3H). ${ }^{13} \mathbf{C}$ NMR (101 MHz, $\left.\mathbf{C D C l}_{3}\right): \delta 163.9,156.0,153.0$, $145.5,133.5,132.2,130.8,129.8,129.5-129.0(\mathrm{~m}), 122.4,120.5,111.6,70.0$, 
$62.0,56.2,42.8,21.7$. HRMS (ESI, m/z): calculated for $\mathrm{C}_{19} \mathrm{H}_{18} \mathrm{ClNO} 6 \mathrm{~S}[\mathrm{M}+\mathrm{H}]^{+}$: 424.0622, found: 424.0617 .

3-(2-(naphthalen-2-yl)-2-tosylacetyl)oxazolidin-2-one

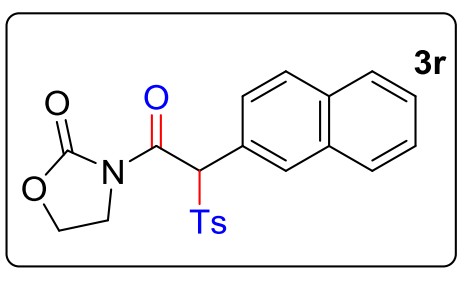

Following the general procedure, $\mathbf{3 r}$ was obtained as a colorless oil (23.7 mg, $0.058 \mathrm{mmol}$, yield: $58 \%) .{ }^{1} \mathbf{H}$ NMR (400 MHz, CDCl $\left.\mathbf{l}_{3}\right): \delta 7.81(\mathrm{~d}, J=8.8 \mathrm{~Hz}, 2 \mathrm{H})$, $7.77-7.71(\mathrm{~m}, 2 \mathrm{H}), 7.56-7.48(\mathrm{~m}, 2 \mathrm{H}), 7.48-7.43(\mathrm{~m}$,

$3 \mathrm{H}), 7.16(\mathrm{~d}, J=8.1 \mathrm{~Hz}, 2 \mathrm{H}), 7.02(\mathrm{~s}, 1 \mathrm{H}), 4.45-4.38(\mathrm{~m}, 1 \mathrm{H}), 4.37-4.29(\mathrm{~m}, 1 \mathrm{H})$, $4.20-4.12(\mathrm{~m}, 1 \mathrm{H}), 4.03-3.94(\mathrm{~m}, 1 \mathrm{H}), 2.39(\mathrm{~s}, 3 \mathrm{H}) ;{ }^{13} \mathbf{C}$ NMR (101 MHz, CDCl $\left.\mathbf{3}\right)$ : $\delta 164.2,153.0,145.3,133.8,133.5,132.8,131.0,129.9,129.3,128.4,128.2$, $127.7,127.6,127.1,126.4,125.2,71.4,62.0,42.8,21.7$. HRMS (ESI, m/z): calculated for $\mathrm{C}_{22} \mathrm{H}_{19} \mathrm{NO}_{5} \mathrm{~S}[\mathrm{M}+\mathrm{H}]^{+}$: 410.1062, found: 410.1064 .

\section{3-(2-(naphthalen-1-yl)-2-tosylacetyl)oxazolidin-2-one}

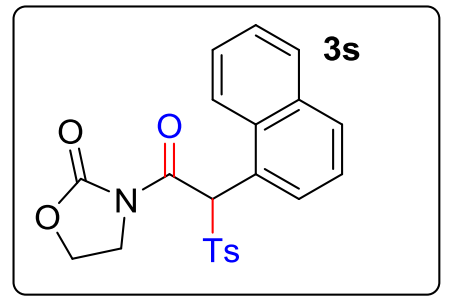

Following the general procedure, $\mathbf{3 s}$ was obtained as a white solid (15.1 mg, $0.037 \mathrm{mmol}$, yield: $37 \%) .{ }^{1} \mathbf{H}$ NMR (401 MHz, $\left.\mathbf{C D C l}_{3}\right) \delta 7.99(\mathrm{~s}, 2 \mathrm{H}), 7.92(\mathrm{~d}, J=7.4 \mathrm{~Hz}, 1 \mathrm{H})$, $7.87(\mathrm{~d}, J=8.2 \mathrm{~Hz}, 1 \mathrm{H}), 7.82-7.78(\mathrm{~m}, 1 \mathrm{H}), 7.47-7.38$

(m, 5H), $7.08(\mathrm{~d}, J=7.7 \mathrm{~Hz}, 2 \mathrm{H}), 4.49-4.39(\mathrm{~m}, 1 \mathrm{H}), 4.38-4.28(\mathrm{~m}, 1 \mathrm{H}), 4.27-4.15$ (m, 1H), $4.05-3.94(\mathrm{~m}, 1 \mathrm{H}), 2.33$ (s, 3H). ${ }^{13} \mathbf{C}$ NMR (101 MHz, CDCl $): \delta 164.4$, 153.3 , 145.2 , 133.7 , $133.6,132.1$, 130.4 , 129.9 , 129.4 , 129.1 , 128.6 , 127.0 , 125.8 , $124.8,123.6,123.1,65.3,62.0,42.9,21.6$. HRMS (ESI, m/z): calculated for $\mathrm{C}_{22} \mathrm{H}_{19} \mathrm{NO}_{5} \mathrm{~S}[\mathrm{M}+\mathrm{H}]^{+}:$410.1062, found: 410.1061 .

\section{3-(2-(thiophen-3-yl)-2-tosylacetyl)oxazolidin-2-one}

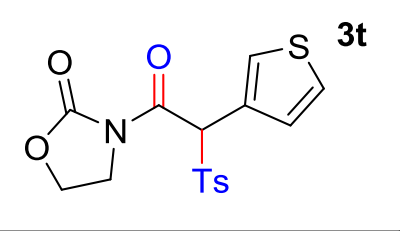

Following the general procedure, $\mathbf{3 t}$ was obtained as a colorless oil $(16.7 \mathrm{mg}, 0.043 \mathrm{mmol}$, yield: $43 \%) .{ }^{1} \mathbf{H}$ NMR (400 MHz, CDCl 3$): \delta 7.46(\mathrm{~d}, J=8.3 \mathrm{~Hz}, 2 \mathrm{H})$, $7.31(\mathrm{~d}, J=3.8 \mathrm{~Hz}, 1 \mathrm{H}), 7.27-7.21(\mathrm{~m}, 3 \mathrm{H}), 7.13(\mathrm{~d}, J$ 
$=6.1 \mathrm{~Hz}, 1 \mathrm{H}), 7.09(\mathrm{~s}, 1 \mathrm{H}), 4.49-4.43(\mathrm{~m}, 1 \mathrm{H}), 4.43-4.37(\mathrm{~m}, 1 \mathrm{H}), 4.22-4.13(\mathrm{~m}$, 1H), $4.07-3.98$ (m, 1H), 2.42 (s, 3H); $\left.{ }^{13} \mathbf{C ~ N M R ~ ( 1 0 1 ~ M H z , ~ C D C l} 3\right): \delta 163.9,153.1$, $145.3,133.5,129.7,129.2,129.0,128.2$, $127.6,125.6,66.9,62.0,42.8,21.7$.

HRMS (ESI, m/z): calculated for $\mathrm{C}_{16} \mathrm{H}_{15} \mathrm{NO}_{5} \mathrm{~S}_{2}[\mathrm{M}+\mathrm{H}]^{+}: 366.0470$, found: 366.0473 .

\section{3-(2-(thiophen-2-yl)-2-tosylacetyl)oxazolidin-2-one}

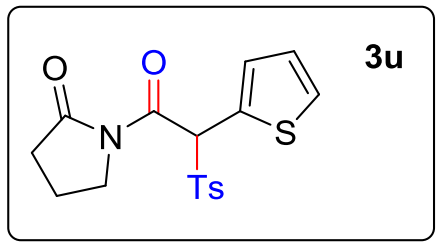

Following the general procedure, $\mathbf{3 u}$ was obtained as a colorless oil (21.8 mg, $0.06 \mathrm{mmol}$, yield: $60 \%)$. ${ }^{1} \mathbf{H}$ NMR (400 MHz, $\left.\mathbf{C D C l}_{3}\right): \delta 7.50(\mathrm{~d}, J=8.3 \mathrm{~Hz}, 2 \mathrm{H}), 7.39(\mathrm{~s}$, $1 \mathrm{H}), 7.33(\mathrm{~d}, J=5.8 \mathrm{~Hz}, 1 \mathrm{H}), 7.23(\mathrm{~d}, J=8.1 \mathrm{~Hz}, 2 \mathrm{H}), 7.02$ $(\mathrm{d}, J=3.6 \mathrm{~Hz}, 1 \mathrm{H}), 6.92(\mathrm{dd}, J=5.1,3.7 \mathrm{~Hz}, 1 \mathrm{H}), 3.94(\mathrm{ddd}, J=11.8,7.9,6.4 \mathrm{~Hz}, 1 \mathrm{H})$, $3.84(\mathrm{ddd}, J=11.8,7.9,6.8 \mathrm{~Hz}, 1 \mathrm{H}), 2.80-2.53(\mathrm{~m}, 2 \mathrm{H}), 2.42(\mathrm{~s}, 3 \mathrm{H}), 2.11-1.97(\mathrm{~m}$, 2H). ${ }^{13} \mathbf{C}$ NMR (101 MHz, $\left.\mathbf{C D C l}_{3}\right): \delta 175.7,164.3,145.3,133.3,130.9,129.9$, $129.2,128.9,128.8,126.4,68.4,46.2,33.6,21.8,16.7$. HRMS (ESI, m/z): calculated for $\mathrm{C}_{17} \mathrm{H}_{17} \mathrm{NO}_{4} \mathrm{~S}_{2}[\mathrm{M}+\mathrm{H}]^{+}: 364.0677$, found: 364.0668 .

\section{1-(2-phenyl-2-tosylacetyl)pyrrolidin-2-one}

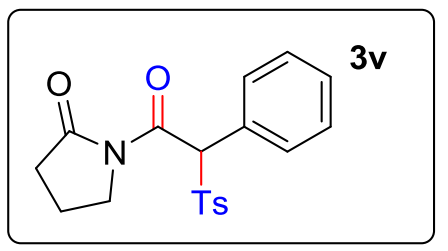

Following the general procedure, $\mathbf{3 v}$ was obtained as a colorless oil (25.7 mg, $0.072 \mathrm{mmol}$, yield: $72 \%) .{ }^{1} \mathbf{H}$ NMR (401 MHz, $\left.\mathbf{C D C l}_{3}\right): \delta 7.44(\mathrm{~d}, J=8.2 \mathrm{~Hz}, 2 \mathrm{H}), 7.35$ (t, $J$ $=7.9 \mathrm{~Hz}, 3 \mathrm{H}), 7.26(\mathrm{t}, J=7.6 \mathrm{~Hz}, 2 \mathrm{H}), 7.19(\mathrm{~d}, J=8.1 \mathrm{~Hz}$,

2H), $6.90(\mathrm{~s}, 1 \mathrm{H}), 3.97-3.86(\mathrm{~m}, 1 \mathrm{H}), 3.84-3.72(\mathrm{~m}, 1 \mathrm{H}), 2.71-2.44(\mathrm{~m}, 2 \mathrm{H}), 2.40$ (s, 3H), 2.08 - 1.90 (m, 2H); ${ }^{13} \mathbf{C}$ NMR (101 MHz, $\left.\mathbf{C D C l}_{3}\right): \delta 175.5,164.9,145.1$, $134.1,131.2,130.1,129.6,129.2,128.5,128.2,73.0,46.1,33.7,21.8,16.8$.

HRMS (ESI, m/z): calculated for $\mathrm{C}_{19} \mathrm{H}_{19} \mathrm{NO}_{4} \mathrm{~S}[\mathrm{M}+\mathrm{H}]^{+}: 358.1113$, found: 358.1113 . 
3-(2-tosyl-2-(3,4,5-trimethoxyphenyl)acetyl)oxazolidin-2-one

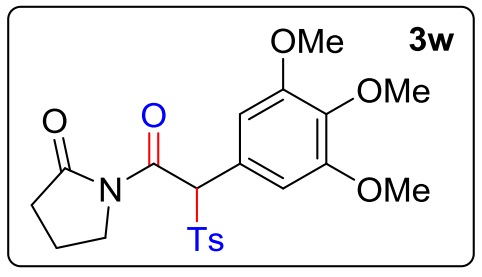

Following the general procedure, $\mathbf{3 w}$ was obtained as a colorless oil (27.4 mg, $0.061 \mathrm{mmol}$, yield: $61 \%) .{ }^{1} \mathbf{H}$ NMR (400 MHz, CDCl $)$ ): $\delta 7.51(\mathrm{~d}, J=8.1 \mathrm{~Hz}, 2 \mathrm{H})$, $7.22(\mathrm{~d}, J=8.1 \mathrm{~Hz}, 2 \mathrm{H}), 6.84(\mathrm{~s}, 1 \mathrm{H}), 6.53(\mathrm{~s}, 2 \mathrm{H}), 3.98$ $-3.87(\mathrm{~m}, 1 \mathrm{H}), 3.81(\mathrm{~s}, 3 \mathrm{H}), 3.79-3.73(\mathrm{~m}, 1 \mathrm{H}), 3.68(\mathrm{~s}, 6 \mathrm{H}), 2.72-2.49(\mathrm{~m}, 2 \mathrm{H})$, 2.40 (s, 3H), 2.10 - $1.94(\mathrm{~m}, 2 \mathrm{H}) ;{ }^{13} \mathbf{C}$ NMR (101 MHz, CDCl $): \delta 175.4,165.0$, $152.8,144.9,138.9,133.8,130.3,128.9,123.2,108.1,72.7,60.8,56.0,46.0$, $33.6,21.6,16.6$. HRMS (ESI, m/z): calculated for $\mathrm{C}_{21} \mathrm{H}_{23} \mathrm{NO}_{8} \mathrm{~S}[\mathrm{M}+\mathrm{H}]^{+}$: 450.1223, found: 450.1221 .

\section{3-(2-phenyl-2-tosylacetyl)thiazolidin-2-one}

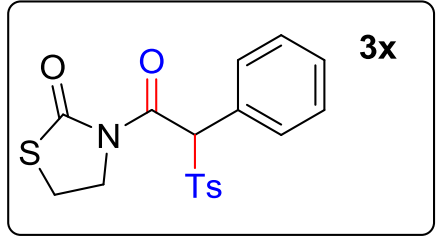

Following the general procedure, $\mathbf{3 x}$ was obtained as a colorless oil (30.0 mg, $0.08 \mathrm{mmol}$, yield: 80\%). ${ }^{1} \mathbf{H}$ NMR (401 MHz, $\left.\mathbf{C D C l}_{3}\right): \delta 7.44(\mathrm{~d}, J=8.2 \mathrm{~Hz}, 2 \mathrm{H}), 7.37-7.31$ (m, 3H), $7.30-7.24(\mathrm{~m}, 2 \mathrm{H}), 7.20(\mathrm{~d}, J=8.1 \mathrm{~Hz}, 2 \mathrm{H}), 6.68$ $(\mathrm{s}, 1 \mathrm{H}), 4.36-4.24(\mathrm{~m}, 1 \mathrm{H}), 4.17-4.01(\mathrm{~m}, 1 \mathrm{H}), 3.43-3.25(\mathrm{~m}, 1 \mathrm{H}), 3.23-3.15(\mathrm{~m}$, 1H), 2.41 (s, 3H). ${ }^{13} \mathbf{C}$ NMR (101 MHz, $\left.\mathbf{C D C l}_{3}\right): \delta 173.0,163.7,145.1,133.9,130.9$, $129.9,129.5,129.1,128.4,127.8,72.9,47.3,24.6,21.7$. HRMS (ESI, m/z): calculated for $\mathrm{C}_{19} \mathrm{H}_{19} \mathrm{NO}_{4} \mathrm{~S}[\mathrm{M}+\mathrm{H}]^{+}: 376.0677$, found: 376.0677 .

\section{1-(2-(4-methoxyphenyl)-2-tosylacetyl)azetidin-2-one}

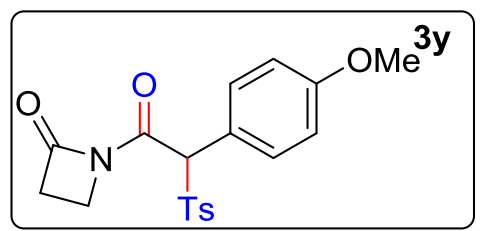

Following the general procedure, $\mathbf{3 y}$ was obtained as a colorless oil (30.0 mg, $0.072 \mathrm{mg}$, yield: $72 \%) .{ }^{1} \mathbf{H}$ NMR (400 MHz, $\left.\mathbf{C D C l}_{3}\right): \delta 7.49(\mathrm{~d}, J=8.1 \mathrm{~Hz}, 2 \mathrm{H}), 7.29$ (d, $J=8.7 \mathrm{~Hz}, 2 \mathrm{H}), 7.22(\mathrm{~d}, J=8.1 \mathrm{~Hz}, 2 \mathrm{H}), 6.80(\mathrm{~d}, J=$ $8.7 \mathrm{~Hz}, 2 \mathrm{H}), 6.01(\mathrm{~s}, 1 \mathrm{H}), 3.79(\mathrm{~s}, 3 \mathrm{H}), 3.70(\mathrm{td}, J=7.3,4.3 \mathrm{~Hz}, 1 \mathrm{H}), 3.57(\mathrm{td}, J=7.2$, $4.4 \mathrm{~Hz}, 1 \mathrm{H}), 3.12$ (ddd, $J=16.5,6.7,4.1 \mathrm{~Hz}, 1 \mathrm{H}), 3.03$ (ddd, $J=16.5,6.6,4.2 \mathrm{~Hz}, 1 \mathrm{H}$ ), $2.42(\mathrm{~s}, 3 \mathrm{H}) ;{ }^{13} \mathbf{C}$ NMR (101 MHz, $\left.\mathbf{C D C l}_{3}\right): \delta 165.1,161.6,160.7,145.2,133.7$, $132.1,129.9,129.2,119.4,113.9,73.8,55.3,37.6,36.6,21.7$. HRMS (ESI, m/z): 
calculated for $\mathrm{C}_{19} \mathrm{H}_{19} \mathrm{NO}_{5} \mathrm{~S}[\mathrm{M}+\mathrm{H}]^{+}:$374.1062, found: 374.1062 .

benzyl phenyl(2-phenyl-2-tosylacetyl)carbamate

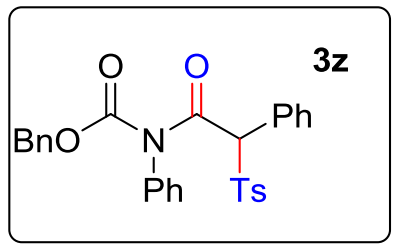

Following the general procedure, $\mathbf{3 z}$ was obtained as a colorless oil (27.0 mg, 0.054 mmol, Yield: 54\%). ${ }^{1} \mathbf{H}$ NMR (400 MHz, CDCl3): $\delta 7.45-7.34(\mathrm{~m}, 6 \mathrm{H}), 7.34-7.24(\mathrm{~m}$, $7 \mathrm{H}), 7.13(\mathrm{~d}, J=8.2 \mathrm{~Hz}, 2 \mathrm{H}), 7.06(\mathrm{ddd}, J=14.7,7.6,2.9 \mathrm{~Hz}$,

4H), 6.75 (s, 1H), 5.12 (s, 2H), 2.38 (s, 3H); ${ }^{13}$ C NMR (101 MHz, CDCl 3 ): $\delta 167.2$, $153.5,144.9,137.2$, 134.6 , 133.4 , $130.8,130.5$, 129.5 , 129.3 , 128.9 , $128.7,128.5$, $128.4,128.3,128.2,127.3,75.0,68.7,21.7$. HRMS (ESI, m/z): calculated for $\mathrm{C}_{29} \mathrm{H}_{25} \mathrm{NO}_{5} \mathrm{~S}[\mathrm{M}+\mathrm{H}]^{+}:$500.1532, found: 500.1531 .

propyl phenyl(2-phenyl-2-tosylacetyl)carbamate

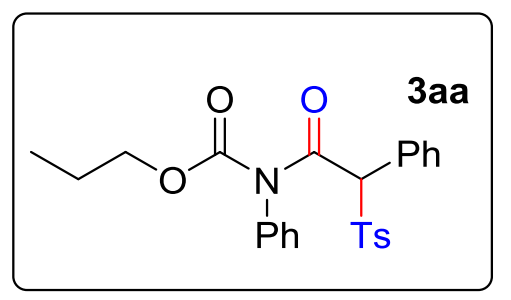

Following the general procedure, 3aa was obtained as a colorless oil (22.0 mg, $0.048 \mathrm{mmol}$, Yield: 48\%). ${ }^{\mathbf{1}} \mathbf{H}$ NMR (400 MHz, CDCl $) \delta 7.42-7.33$ (m, 8H), 7.29 $(\mathrm{d}, J=7.4 \mathrm{~Hz}, 2 \mathrm{H}), 7.14(\mathrm{~d}, J=8.1 \mathrm{~Hz}, 2 \mathrm{H}), 7.09(\mathrm{dd}$, $J=7.9,1.6 \mathrm{~Hz}, 2 \mathrm{H}), 6.79(\mathrm{~s}, 1 \mathrm{H}), 4.09-4.04(\mathrm{~m}, 1 \mathrm{H}), 4.03-3.99(\mathrm{~m}, 1 \mathrm{H}), 2.38(\mathrm{~s}$, 3H), 1.48 (q, $J=6.9 \mathrm{~Hz}, 2 \mathrm{H}), 0.69(\mathrm{t}, J=7.4 \mathrm{~Hz}, 3 \mathrm{H}) ;{ }^{13} \mathbf{C} \mathbf{N M R}\left(\mathbf{1 0 1} \mathbf{M H z}, \mathbf{C D C l}_{3}\right) \delta$ $167.3,153.8,144.8,137.4,133.4,130.8$, 130.5 , 129.4 , 129.2 , 128.8 , 128.6 , 128.4 , $128.4,128.1,75.0,69.1,21.6,10.0$. HRMS (ESI, m/z): calculated for $\mathrm{C}_{25} \mathrm{H}_{25} \mathrm{NO}_{5} \mathrm{~S}$ $[\mathrm{M}+\mathrm{H}]^{+}:$452.1532, found: 452.1530 .

propyl (4-phenoxyphenyl)(2-phenyl-2-tosylacetyl)carbamate

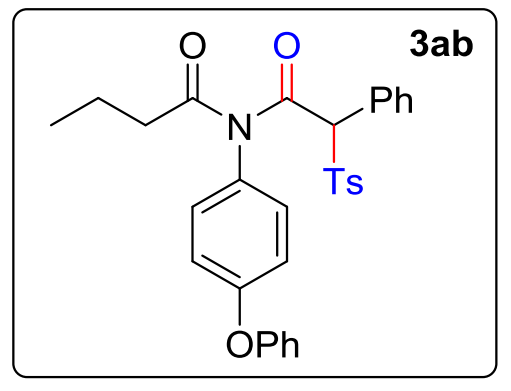

Following the general procedure, $\mathbf{3 a b}$ was obtained as a colorless oil (24.0 mg, $0.024 \mathrm{mmol}$, Yield: $45 \%) .{ }^{1} \mathbf{H}$

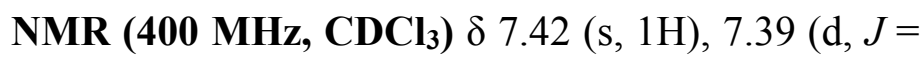
$4.0 \mathrm{~Hz}, 2 \mathrm{H}), 7.38-7.33(\mathrm{~m}, 5 \mathrm{H}), 7.31-7.27(\mathrm{~m}, 2 \mathrm{H})$, $7.17-7.13(\mathrm{~m}, 3 \mathrm{H}), 7.06(\mathrm{~d}, J=1.1 \mathrm{~Hz}, 1 \mathrm{H}), 7.04(\mathrm{dd}$, $J=1.9,0.8 \mathrm{~Hz}, 1 \mathrm{H}), 7.02(\mathrm{t}, J=4.9 \mathrm{~Hz}, 1 \mathrm{H}), 7.01(\mathrm{~s}, 1 \mathrm{H}), 7.01(\mathrm{t}, J=4.7 \mathrm{~Hz}, 1 \mathrm{H}), 6.80$ (s, 1H), $4.12-4.06(\mathrm{~m}, 1 \mathrm{H}), 4.06-4.00(\mathrm{~m}, 1 \mathrm{H}), 2.38(\mathrm{~s}, 3 \mathrm{H}), 1.51(\mathrm{~h}, J=7.3 \mathrm{~Hz}, 2 \mathrm{H})$, $0.73(\mathrm{t}, J=7.4 \mathrm{~Hz}, 3 \mathrm{H}) ;{ }^{13} \mathbf{C}$ NMR (101 MHz, CDCl $\left.\mathbf{3}\right) \delta 167.5,157.5,156.3,153.8$, $144.9,133.4,132.0,130.7,130.4,129.9$, $129.4,129.4,128.9,128.4,128.4,123.9$, 
$119.5,118.8,75.0,69.1,21.6,10.0$.HRMS (ESI, m/z): calculated for $\mathrm{C}_{31} \mathrm{H}_{29} \mathrm{NO}_{5} \mathrm{~S}$ $[\mathrm{M}+\mathrm{H}]^{+}:$528.1845, found: 528.1845 .

(4S)-4-benzyl-3-(2-phenyl-2-tosylacetyl)oxazolidin-2-one

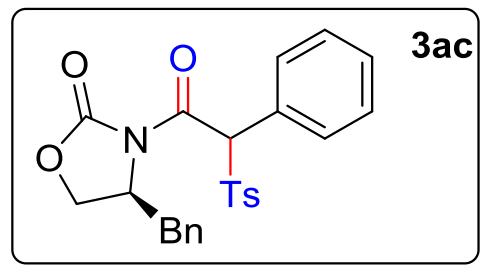

Following the general procedure, 3ac was obtained as a colorless oil (23.8 mg, $0.053 \mathrm{mmol}$, yield: $53 \%) .{ }^{1} \mathbf{H}$ NMR (400 MHz, CDCl $): \delta 7.54(\mathrm{~d}, J=8.2 \mathrm{~Hz}, 1 \mathrm{H})$, $7.47(\mathrm{~d}, J=8.3 \mathrm{~Hz}, 2 \mathrm{H}), 7.45-7.39$ (m, 3.5H), $7.39-$ $7.33(\mathrm{~m}, 3 \mathrm{H}), 7.32-7.27(\mathrm{~m}, 3 \mathrm{H}), 7.27-7.17$ (m, 7.5H), 7.02 (dd, J = 6.4, $2.5 \mathrm{~Hz}$, $1.5 \mathrm{H}), 6.93(\mathrm{~s}, 1 \mathrm{H}), 6.83(\mathrm{~s}, 1 \mathrm{H}), 4.86-4.75(\mathrm{~m}, 1 \mathrm{H}), 4.71-4.64(\mathrm{~m}, 0.5 \mathrm{H}), 4.24(\mathrm{t}, J$ $=8.4 \mathrm{~Hz}, 1 \mathrm{H}), 4.15(\mathrm{dd}, J=6.9,2.3 \mathrm{~Hz}, 1.5 \mathrm{H}), 4.12-4.07(\mathrm{~m}, 0.5 \mathrm{H}), 3.35(\mathrm{dd}, J=$ 13.6, 3.2 Hz, 0.5H), $3.16(\mathrm{dd}, J=13.5,3.2 \mathrm{~Hz}, 1 \mathrm{H}), 2.80(\mathrm{dd}, J=13.6,9.5 \mathrm{~Hz}, 0.5 \mathrm{H})$, $2.66(\mathrm{dd}, J=13.5,9.3 \mathrm{~Hz}, 1 \mathrm{H}), 2.41(\mathrm{~s}, 4.5 \mathrm{H}) ;{ }^{13} \mathbf{C}$ NMR (101 MHz, $\left.\mathbf{C D C l}_{3}\right) \delta 164.1$, $163.9,152.9,152.9,145.2$, 145.2 , 134.8, 134.4 , 134.1 , 133.9, 131.1 , 131.0, 129.8 , $129.8,129.7,129.7,129.5,129.3,129.3$, 129.2 , 129.0 , 128.9 , 128.5 , 128.5 , 127.7 , $127.6,127.4,127.4,71.5,71.3,66.2,65.9,55.5,55.4,37.4,37.2,21.7$. HRMS (ESI, m/z): calculated for $\mathrm{C}_{25} \mathrm{H}_{23} \mathrm{NO}_{5} \mathrm{~S}[\mathrm{M}+\mathrm{H}]^{+}: 480.1481$, found: 480.1475 .

(4S,5R)-3-(2-(4-methoxyphenyl)-2-tosylacetyl)-4-methyl-5-phenyloxazolidin-2one

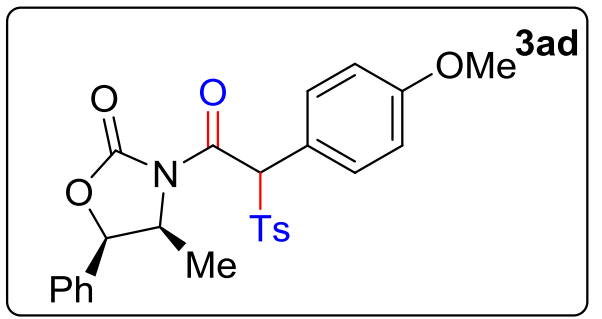

Following the general procedure, 3ad was obtained as a colorless oil (37.9 mg, $0.079 \mathrm{mmol}$, yield: 79\%). ${ }^{1} \mathrm{H}$ NMR (400 $\left.\mathbf{M H z}, \mathrm{CDCl}_{3}\right): \delta$ $7.47(\mathrm{~d}, J=8.1 \mathrm{~Hz}, 2 \mathrm{H}), 7.40(\mathrm{~d}, J=8.2 \mathrm{~Hz}, 2 \mathrm{H})$, $7.33(\mathrm{~d}, J=7.5 \mathrm{~Hz}, 3 \mathrm{H}), 7.29(\mathrm{~d}, J=8.8 \mathrm{~Hz}, 3 \mathrm{H})$, $7.24(\mathrm{~s}, 1 \mathrm{H}), 7.22(\mathrm{~s}, 1 \mathrm{H}), 7.17(\mathrm{dt}, J=8.5,5.2 \mathrm{~Hz}, 8 \mathrm{H}), 6.83(\mathrm{~s}, 1 \mathrm{H}), 6.75(\mathrm{t}, J=8.8$ $\mathrm{Hz}, 4 \mathrm{H}), 6.67$ (s, 1H), 5.64 (d, $J=7.2 \mathrm{~Hz}, 1 \mathrm{H}), 5.48$ (d, $J=7.4 \mathrm{~Hz}, 1 \mathrm{H}), 4.85-4.75$ (m, 1H), $4.75-4.62(\mathrm{~m}, 1 \mathrm{H}), 3.73(\mathrm{~d}, J=3.3 \mathrm{~Hz}, 6 \mathrm{H}), 2.34(\mathrm{~d}, J=2.9 \mathrm{~Hz}, 6 \mathrm{H}), 0.84$ $(\mathrm{d}, J=6.6 \mathrm{~Hz}, 3 \mathrm{H}), 0.73(\mathrm{~d}, J=6.6 \mathrm{~Hz}, 3 \mathrm{H}) ;{ }^{13} \mathbf{C} \mathbf{N M R}\left(\mathbf{1 0 1} \mathbf{M H z}, \mathbf{C D C l}_{3}\right): \delta 164.2$, $163.9,160.7,160.6,152.6,152.5,145.1,145.0,134.2,133.9,132.7,132.6,132.3$, $129.9,129.8,129.2,129.2,129.0,128.9,128.7,128.7,125.7,125.6,119.5,119.5$, 
$114.0,113.9,79.2,78.8,71.2,70.7,55.4,55.3,55.3,55.1,21.7,21.7,14.3,14.1$.

HRMS (ESI, m/z): calculated for $\mathrm{C}_{26} \mathrm{H}_{25} \mathrm{NO}_{6} \mathrm{~S}[\mathrm{M}+\mathrm{H}]^{+}: 480.1481$, found: 480.1482 .

\section{3-(2-(cyclohex-1-en-1-yl)-2-tosylacetyl)oxazolidin-2-one}

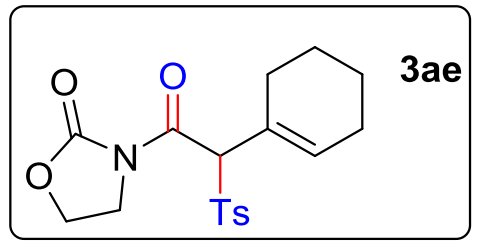

Following the general procedure, 3ae was obtained as a colorless oil (24.7 mg, $0.068 \mathrm{mmol}$, yield: $68 \%) .{ }^{1} \mathbf{H}$ NMR (400 MHz, $\left.\mathbf{C D C l}_{3}\right): \delta 7.71(\mathrm{~d}, J=8.2 \mathrm{~Hz}, 2 \mathrm{H})$, $7.31(\mathrm{~d}, J=8.1 \mathrm{~Hz}, 2 \mathrm{H}), 6.47(\mathrm{~d}, J=1.8 \mathrm{~Hz}, 1 \mathrm{H}), 4.45$ $-4.29(\mathrm{~m}, 2 \mathrm{H}), 4.08-3.93(\mathrm{~m}, 2 \mathrm{H}), 3.72(\mathrm{~d}, J=5.8 \mathrm{~Hz}, 1 \mathrm{H}), 3.51(\mathrm{~d}, J=14.9 \mathrm{~Hz}, 1 \mathrm{H})$, $2.76-2.55(\mathrm{~m}, 2 \mathrm{H}), 2.43(\mathrm{~s}, 3 \mathrm{H}), 2.24-2.05(\mathrm{~m}, 1 \mathrm{H}), 1.95(\mathrm{~d}, J=13.3 \mathrm{~Hz}, 1 \mathrm{H}), 1.89$ - $1.77(\mathrm{~m}, 1 \mathrm{H}), 1.69-1.58(\mathrm{~m}, 1 \mathrm{H}), 1.44-1.30(\mathrm{~m}, 1 \mathrm{H}) ;{ }^{13} \mathbf{C}$ NMR (101 MHz, CDCl$)$ $\delta 163.9,153.2,153.1,144.9,134.7,129.7,128.9,121.5,70.1,62.0,42.6,27.5$, $27.0,26.4,21.8,21.1$. HRMS (ESI, m/z): calculated for $\mathrm{C}_{18} \mathrm{H}_{21} \mathrm{NO}_{5} \mathrm{~S}[\mathrm{M}+\mathrm{H}]^{+}$: 364.1219, found: 364.1213 .

\section{3-(2-cyclopropyl-2-tosylacetyl)oxazolidin-2-one}

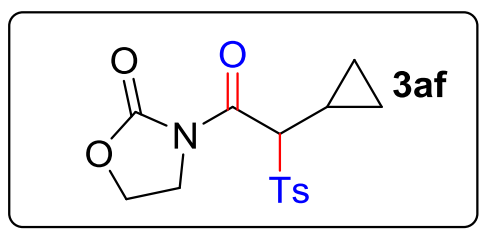

3aeFollowing the general procedure, 3af was obtained as a colorless oil (16.2 mg, $0.05 \mathrm{mmol}$, yield: $50 \%) .{ }^{1} \mathbf{H}$ NMR (400 MHz, $\left.\mathbf{C D C l}_{3}\right): \delta 7.85(\mathrm{~d}, J=8.2 \mathrm{~Hz}, 2 \mathrm{H})$, $7.36(\mathrm{~d}, J=8.3 \mathrm{~Hz}, 2 \mathrm{H}), 5.16(\mathrm{~d}, J=10.5 \mathrm{~Hz}, 1 \mathrm{H}), 4.58$ $-4.36(\mathrm{~m}, 2 \mathrm{H}), 4.19-4.12(\mathrm{~m}, 1 \mathrm{H}), 4.12-4.04(\mathrm{~m}, 1 \mathrm{H}), 2.47(\mathrm{~s}, 3 \mathrm{H}), 1.47-1.30(\mathrm{~m}$, 1H), $0.76-0.50(\mathrm{~m}, 2 \mathrm{H}), 0.44-0.18(\mathrm{~m}, 2 \mathrm{H}) ;{ }^{13} \mathbf{C}$ NMR (101 MHz, CDCl $): \delta 165.8$, $153.4,145.3,135.1,129.6,129.5,70.8,62.0,42.9,21.7,9.0,4.9,3.6$. HRMS (ESI, m/z): calculated for $\mathrm{C}_{15} \mathrm{H}_{17} \mathrm{NO}_{5} \mathrm{~S}[\mathrm{M}+\mathrm{H}]^{+}:$324.0906, found: 324.0907 .

\section{3-(2-tosylhexanoyl)oxazolidin-2-one}

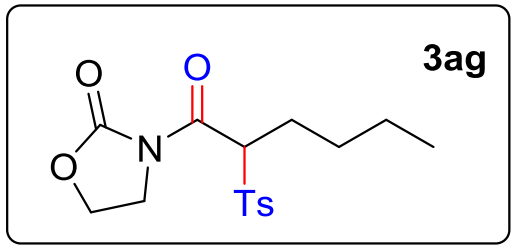

Following the general procedure, $\mathbf{3 a g}$ was obtained as a colorless oil ( $8.9 \mathrm{mg}, 0.026 \mathrm{mmol}$, yield: $26 \%) .{ }^{1} \mathbf{H}$

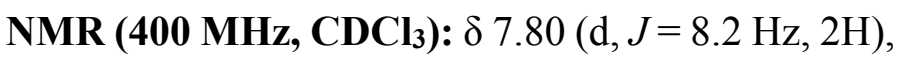
$7.37(\mathrm{~d}, J=8.1 \mathrm{~Hz}, 2 \mathrm{H}), 5.90(\mathrm{dd}, J=11.2,3.4 \mathrm{~Hz}$, 
1H), $4.54-4.30(\mathrm{~m}, 2 \mathrm{H}), 4.14-4.01(\mathrm{~m}, 2 \mathrm{H}), 2.47(\mathrm{~s}, 3 \mathrm{H}), 2.22-2.05(\mathrm{~m}, 1 \mathrm{H}), 1.98$ $-1.74(\mathrm{~m}, 1 \mathrm{H}), 1.40-1.11(\mathrm{~m}, 4 \mathrm{H}), 0.96-0.73(\mathrm{~m}, 3 \mathrm{H}) ;{ }^{13} \mathbf{C}$ NMR (101 MHz, CDCl$\left.)_{3}\right)$ $\delta 165.4,153.2,145.4,134.5,129.7,129.3,66.1,61.9,43.0,28.7,26.9,22.2$, $21.7,13.6$.HRMS (ESI, m/z): calculated for $\mathrm{C}_{16} \mathrm{H}_{21} \mathrm{NO}_{5} \mathrm{~S}[\mathrm{M}+\mathrm{H}]^{+}: 340.1219$, found: 340.1219 .

\section{3-(2-tosylheptanoyl)oxazolidin-2-one}

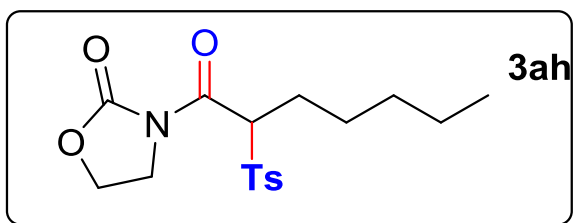

Following the general procedure, 3ah was obtained as a colorless oil $(7.1 \mathrm{mg}, 0.02 \mathrm{mmol}$, yield: 20\%). ${ }^{1}$ H NMR (400 MHz, $\left.\mathbf{C D C l}_{3}\right): \delta 7.80$ (d, $J=8.3 \mathrm{~Hz}, 2 \mathrm{H}), 7.37(\mathrm{~d}, J=8.1 \mathrm{~Hz}, 2 \mathrm{H}), 5.89$ (dd, $J=11.2,3.4 \mathrm{~Hz}, 1 \mathrm{H}), 4.56-4.31(\mathrm{~m}, 2 \mathrm{H}), 4.17-3.99$ (m, 2H), 2.47 (s, 3H), 2.19 - $2.01(\mathrm{~m}, 1 \mathrm{H}), 1.92-1.75(\mathrm{~m}, 1 \mathrm{H}), 1.28-1.14(\mathrm{~m}, 6 \mathrm{H}), 0.82(\mathrm{t}, J=6.2 \mathrm{~Hz}, 3 \mathrm{H}) ;{ }^{13} \mathrm{C}$ NMR (101 MHz, CDCl $\mathbf{3}): \delta 165.4,153.2,145.4,134.6,129.7,129.3$, 66.1 , 61.9 , $43.0,31.2,27.2,26.3,22.2,21.7,13.8$. HRMS (ESI, m/z): calculated for $\mathrm{C}_{17} \mathrm{H}_{23} \mathrm{NO}_{5} \mathrm{~S}[\mathrm{M}+\mathrm{H}]^{+}:$354.1375, found: 354.1371 .

\section{3-(5-phenyl-2-tosylpentanoyl)oxazolidin-2-one}

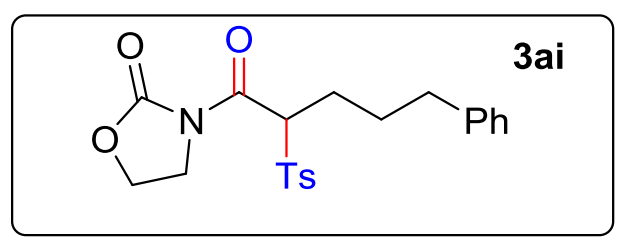

Following the general procedure, 3ai was obtained as a colorless oil $(11.2 \mathrm{mg}, 0.028$ mmol, yield: 28\%). ${ }^{1}$ H NMR (400 MHz, $\left.\mathbf{C D C l}_{3}\right): \delta 7.77(\mathrm{~d}, J=8.2 \mathrm{~Hz}, 2 \mathrm{H}), 7.35(\mathrm{~d}, J=$ $8.1 \mathrm{~Hz}, 2 \mathrm{H}), 7.22$ (t, $J=7.3 \mathrm{~Hz}, 2 \mathrm{H}), 7.15$ (t, $J=7.2 \mathrm{~Hz}, 1 \mathrm{H}), 7.05(\mathrm{~d}, J=7.2 \mathrm{~Hz}, 2 \mathrm{H})$, $5.94(\mathrm{dd}, J=11.1,3.5 \mathrm{~Hz}, 1 \mathrm{H}), 4.60-4.23(\mathrm{~m}, 2 \mathrm{H}), 4.17-3.86(\mathrm{~m}, 2 \mathrm{H}), 2.62-2.48$ (m, 2H), 2.47 (s, 3H), $2.27-2.10(\mathrm{~m}, 1 \mathrm{H}), 1.95-1.79(\mathrm{~m}, 1 \mathrm{H}), 1.63-1.46(\mathrm{~m}, 2 \mathrm{H})$; ${ }^{13}$ C NMR (101 MHz, CDCl 3 ): $\delta 165.2,153.2,145.4,141.0,134.4,129.7,129.3$, $128.4,128.3,126.0,65.8,61.9,42.95,35.1,28.4,26.8,21.7$. HRMS (ESI, m/z): calculated for $\mathrm{C}_{21} \mathrm{H}_{23} \mathrm{NO}_{5} \mathrm{~S}[\mathrm{M}+\mathrm{H}]^{+}:$402.1375, found: 402.1375 . 


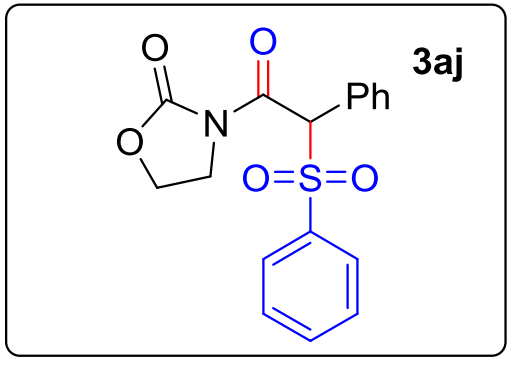

Following the general procedure, 3aj was obtained as a colorless oil (25.5 mg, $0.074 \mathrm{mmol}$, yield: $74 \%) .{ }^{1} \mathbf{H}$ NMR (400 MHz, CDCl 3$): \delta 7.59(\mathrm{t}, 3 \mathrm{H}), 7.42(\mathrm{t}, J=$ $7.9 \mathrm{~Hz}, 2 \mathrm{H}), 7.38-7.34(\mathrm{~m}, 3 \mathrm{H}), 7.27(\mathrm{t}, J=7.6 \mathrm{~Hz}$, $2 \mathrm{H}), 6.89(\mathrm{~s}, 1 \mathrm{H}), 4.47-4.33(\mathrm{~m}, 2 \mathrm{H}), 4.20-4.11(\mathrm{~m}$,

1H), 4.05 - $3.94(\mathrm{~m}, 1 \mathrm{H}) ;{ }^{13} \mathbf{C}$ NMR (101 MHz, $\left.\mathbf{C D C l}_{3}\right): \delta 164.0,153.0,136.7,134.1$, $130.9,129.8,129.7,128.5,128.5,127.6,71.3,62.0,42.8$. HRMS (ESI, m/z): calculated for $\mathrm{C}_{17} \mathrm{H}_{15} \mathrm{NO}_{5} \mathrm{~S}[\mathrm{M}+\mathrm{H}]^{+}: 346.0749$, found: 346.0748 .

\section{3-(2-((4-fluorophenyl)sulfonyl)-2-phenylacetyl)oxazolidin-2-one}

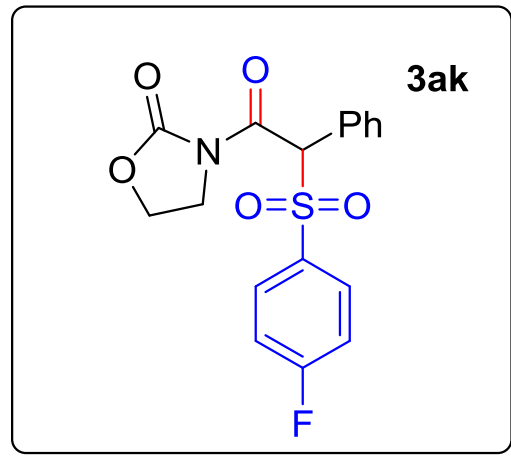

Following the general procedure, 3ak was obtained as a colorless oil (27.6 mg, $0.076 \mathrm{mmol}$, yield: $76 \%) .{ }^{1} \mathbf{H}$ NMR (400 MHz, CDCl $): \delta 7.57(\mathrm{dd}, J=8.7,5.1 \mathrm{~Hz}$, 2H), $7.38(\mathrm{t}, J=7.1 \mathrm{~Hz}, 1 \mathrm{H}), 7.33(\mathrm{~d}, J=7.1 \mathrm{~Hz}, 2 \mathrm{H})$, $7.31-7.27(\mathrm{~m}, 2 \mathrm{H}), 7.08(\mathrm{t}, J=8.5 \mathrm{~Hz}, 2 \mathrm{H}), 6.87(\mathrm{~s}$, $1 \mathrm{H}), 4.50-4.34(\mathrm{~m}, 2 \mathrm{H}), 4.22-4.11(\mathrm{~m}, 1 \mathrm{H}), 4.05-$ $3.94(\mathrm{~m}, 1 \mathrm{H}) ;{ }^{13} \mathbf{C}$ NMR (101 MHz, $\left.\mathbf{C D C l}_{3}\right): \delta 166.0$ $(\mathrm{d}, J=257.2 \mathrm{~Hz}), 164.0,152.9,132.9(\mathrm{~d}, J=9.8 \mathrm{~Hz}), 132.4(\mathrm{~d}, J=3.1 \mathrm{~Hz}), 130.8$, $129.8,128.6,127.5,115.8(\mathrm{~d}, J=22.7 \mathrm{~Hz}), 71.5,62.0,42.7$. HRMS (ESI, m/z): calculated for $\mathrm{C}_{17} \mathrm{H}_{14} \mathrm{FNO}_{5} \mathrm{~S}[\mathrm{M}+\mathrm{H}]^{+}:$364.0655, found: 364.0656 .

\section{3-(2-((4-chlorophenyl)sulfonyl)-2-phenylacetyl)oxazolidin-2-one}

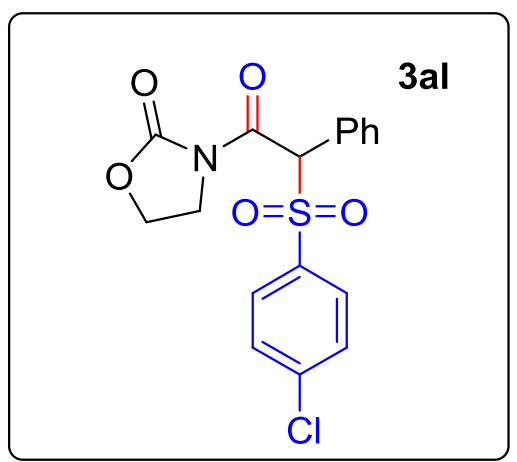

Following the general procedure, 3al was obtained as a colorless oil (23.5 mg, $0.062 \mathrm{mmol}$, yield: $62 \%) .{ }^{\mathbf{1}} \mathbf{H}$ NMR (400 MHz, CDCl 3$): \delta 7.49(\mathrm{~d}, J=8.6 \mathrm{~Hz}, 2 \mathrm{H})$, $7.38(\mathrm{~d}, J=8.6 \mathrm{~Hz}, 3 \mathrm{H}), 7.34(\mathrm{~d}, J=7.1 \mathrm{~Hz}, 2 \mathrm{H}), 7.30$ $(\mathrm{d}, J=7.3 \mathrm{~Hz}, 2 \mathrm{H}), 6.87(\mathrm{~s}, 1 \mathrm{H}), 4.51-4.33(\mathrm{~m}, 2 \mathrm{H})$, $4.23-4.11(\mathrm{~m}, 1 \mathrm{H}), 4.04-3.94(\mathrm{~m}, 1 \mathrm{H}) ;{ }^{13} \mathbf{C}$ NMR (101 MHz, $\left.\mathbf{C D C l}_{3}\right): \delta 163.9,152.9,140.9,135.0$, 
$131.4,130.8,129.9,128.8,128.6,127.4,71.5,62.0,42.7$. HRMS (ESI, m/z): calculated for $\mathrm{C}_{17} \mathrm{H}_{14} \mathrm{ClNO}_{5} \mathrm{~S}[\mathrm{M}+\mathrm{H}]^{+}: 380.0359$, found: 380.0357 .

\section{3-(2-((4-methoxyphenyl)sulfonyl)-2-phenylacetyl)oxazolidin-2-one}

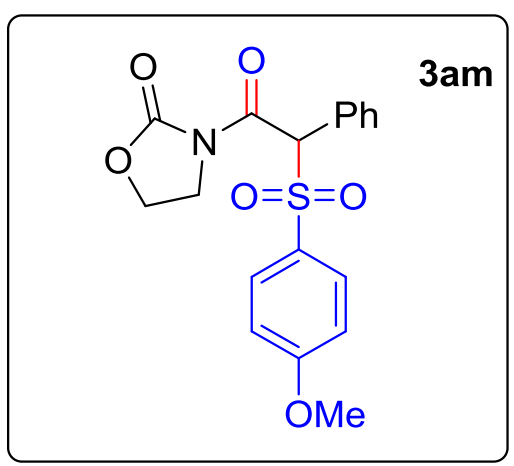

Following the general procedure, 3am was obtained as a white solid (20.0 mg, $0.053 \mathrm{mmol}$, yield: $53 \%)$. ${ }^{1}$ H NMR (400 MHz, $\left.\mathbf{C D C l}_{3}\right): \delta 7.49(\mathrm{~d}, J=9.0 \mathrm{~Hz}$, $2 \mathrm{H}), 7.39-7.33(\mathrm{~m}, 3 \mathrm{H}), 7.28(\mathrm{~d}, J=7.4 \mathrm{~Hz}, 2 \mathrm{H})$, $6.87(\mathrm{~s}, 1 \mathrm{H}), 6.85(\mathrm{~d}, J=2.6 \mathrm{~Hz}, 2 \mathrm{H}), 4.46-4.40(\mathrm{~m}$, $1 \mathrm{H}), 4.40-4.33(\mathrm{~m}, 1 \mathrm{H}), 4.16$ (ddd, $J=11.0,9.3,6.2$ Hz, 1H), 3.99 (ddd, $J=11.1,9.3,7.4 \mathrm{~Hz}, 1 \mathrm{H}), 3.85$ (s, 3H); ${ }^{13}$ C NMR (101 MHz, $\left.\mathbf{C D C l}_{3}\right): \delta 164.2,164.0,153.0,132.1,130.9,129.6$, $128.5,128.1,127.9,113.7,71.4,62.0,55.6,42.8$. HRMS (ESI, m/z): calculated for $\mathrm{C}_{18} \mathrm{H}_{17} \mathrm{NO}_{6} \mathrm{~S}[\mathrm{M}+\mathrm{H}]^{+}:$376.0855, found: 376.0852 .

\section{3-(2-([1,1'-biphenyl]-4-ylsulfonyl)-2-phenylacetyl)oxazolidin-2-one}

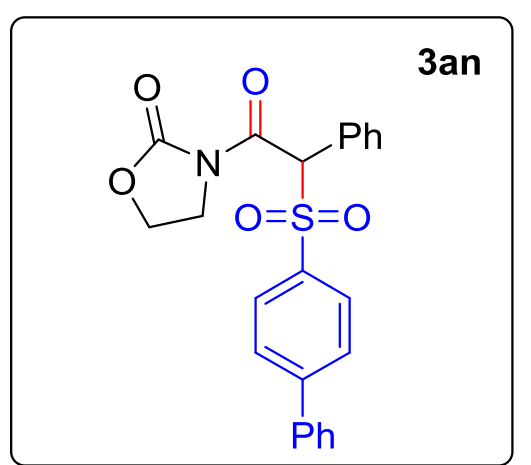

Following the general procedure, 3an was obtained as a white solid (32.8 mg, $0.078 \mathrm{mmol}$, yield: $78 \%$ ). ${ }^{\mathbf{1}} \mathbf{H}$ NMR (400 MHz, CDCl 3$): \delta 7.63(\mathrm{~s}, 4 \mathrm{H}), 7.60(\mathrm{~d}, J=$ $7.3 \mathrm{~Hz}, 2 \mathrm{H}), 7.48(\mathrm{t}, J=7.3 \mathrm{~Hz}, 2 \mathrm{H}), 7.45-7.35(\mathrm{~m}$, 4H), $7.29(\mathrm{~d}, J=7.6 \mathrm{~Hz}, 2 \mathrm{H}), 6.92(\mathrm{~s}, 1 \mathrm{H}), 4.48-4.32$ $(\mathrm{m}, 2 \mathrm{H}), 4.21-4.12(\mathrm{~m}, 1 \mathrm{H}), 4.04-3.95(\mathrm{~m}, 1 \mathrm{H}) ;{ }^{13} \mathrm{C}$ NMR (101 MHz, CDCl3): $\delta 164.0,153.0,146.8$, $138.9,135.2$, $131.0,130.4,129.7,129.0$, 128.7 , 128.5 , $127.6,127.3$, $127.0,71.4$, $62.0,42.8$. HRMS (ESI, m/z): calculated for $\mathrm{C}_{23} \mathrm{H}_{19} \mathrm{NO}_{5} \mathrm{~S}[\mathrm{M}+\mathrm{H}]^{+}: 422.1062$, found: 422.1058 . 
3-(2-(naphthalen-2-ylsulfonyl)-2-phenylacetyl)oxazolidin-2-one

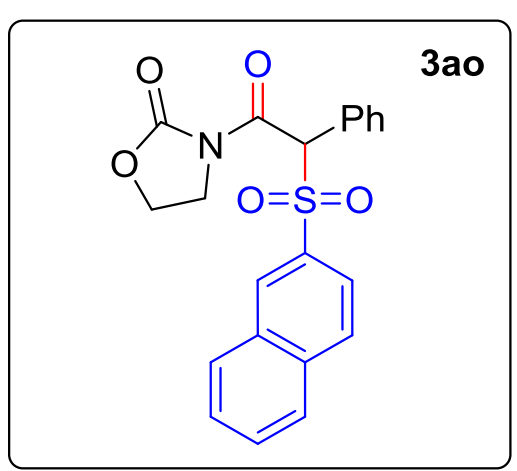

Following the general procedure, 3ao was obtained as a white solid (20.5 mg, $0.052 \mathrm{mmol}$, yield: $52 \%) .{ }^{1} \mathbf{H}$ NMR (400 MHz, $\left.\mathbf{C D C l}_{3}\right): \delta 8.11(\mathrm{~s}, 1 \mathrm{H}), 7.94-7.80$ $(\mathrm{m}, 3 \mathrm{H}), 7.66(\mathrm{t}, J=7.5 \mathrm{~Hz}, 1 \mathrm{H}), 7.62-7.55(\mathrm{~m}, 2 \mathrm{H})$, $7.35(\mathrm{t}, 3 \mathrm{H}), 7.23(\mathrm{t}, J=7.6 \mathrm{~Hz}, 2 \mathrm{H}), 6.96(\mathrm{~s}, 1 \mathrm{H}), 4.45$ $-4.29(\mathrm{~m}, 2 \mathrm{H}), 4.22-4.07(\mathrm{~m}, 1 \mathrm{H}), 4.03-3.92(\mathrm{~m}$,

1H); ${ }^{13} \mathbf{C}$ NMR (101 MHz, $\left.\mathbf{C D C l}_{3}\right): \delta 164.0,153.0$, $135.4,133.6$, 132.0 , $131.6,131.0$, 129.7 , 129.5 , 129.5 , 128.6 , 128.5 , 127.8 , 127.6 , $127.5,124.1,71.4,62.0,42.7$. HRMS (ESI, m/z): calculated for $\mathrm{C}_{21} \mathrm{H}_{17} \mathrm{NO}_{5} \mathrm{~S}$ $[\mathrm{M}+\mathrm{H}]^{+}:$369.4370, found: 369.4370 .

\section{3-(2-(ethylsulfonyl)-2-phenylacetyl)oxazolidin-2-one}

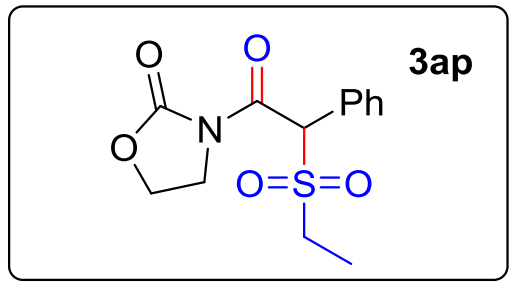

Following the general procedure, 3ap was obtained as a colorless oil (11.9 mg, $0.04 \mathrm{mmol}$, yield: 40\%). ${ }^{1} \mathbf{H}$ NMR (400 MHz, $\left.\mathbf{C D C l}_{3}\right): \delta 7.68-7.59(\mathrm{~m}, 2 \mathrm{H}), 7.46$ $-7.35(\mathrm{~m}, 3 \mathrm{H}), 6.80(\mathrm{~s}, 1 \mathrm{H}), 4.50-4.45(\mathrm{~m}, 1 \mathrm{H}), 4.44$ - $4.38(\mathrm{~m}, 1 \mathrm{H}), 4.24-4.13(\mathrm{~m}, 1 \mathrm{H}), 4.10-3.99(\mathrm{~m}, 1 \mathrm{H}), 3.31-3.19(\mathrm{~m}, 1 \mathrm{H}), 3.05-$ $2.91(\mathrm{~m}, 1 \mathrm{H}), 1.30$ (t, $J=7.5 \mathrm{~Hz}, 3 \mathrm{H}) ;{ }^{13} \mathbf{C}$ NMR (101 MHz, $\left.\mathbf{C D C l}_{3}\right): \delta 164.4,152.9$, $130.4,130.0,129.1,127.7,69.4,62.0,45.2,42.7,5.4$. HRMS (ESI, m/z): calculated for $\mathrm{C}_{13} \mathrm{H}_{15} \mathrm{NO}_{5} \mathrm{~S}[\mathrm{M}+\mathrm{H}]^{+}:$298.0749, found: 298.0753 .

\section{3-(2-(methylsulfonyl)-2-phenylacetyl)oxazolidin-2-one}

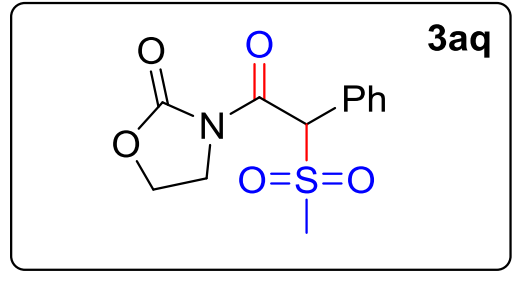

Following the general procedure, $\mathbf{3 a q}$ was obtained as a colorless oil (11.9 mg, $0.056 \mathrm{mmol}$, yield: $56 \%) .{ }^{1} \mathbf{H}$ NMR (400 MHz, $\left.\mathbf{C D C l}_{3}\right): \delta 7.62(\mathrm{dd}, J=6.4,2.9 \mathrm{~Hz}$, 2H), $7.44(\mathrm{dd}, J=5.0,1.7 \mathrm{~Hz}, 3 \mathrm{H}), 6.66(\mathrm{~s}, 1 \mathrm{H}), 4.55$ - 4.35 (m, 2H), 4.26 - 4.15 (m, 1H), $4.13-3.99$ (m, 1H), 2.97 (s, 3H); ${ }^{13}$ C NMR (101 MHz, CDCl 3$): \delta 164.8,152.7,130.2,130.0,129.3,127.8,71.1,62.1,42.6,38.3$. HRMS (ESI, m/z): calculated for $\mathrm{C}_{12} \mathrm{H}_{13} \mathrm{NO}_{5} \mathrm{~S}[\mathrm{M}+\mathrm{H}]^{+}: 284.0593$, found: 284.0593 . 
Methyl 4-(1-([1,1'-biphenyl]-4-ylsulfonyl)-2-oxo-2-(2-oxooxazolidin-3-yl)ethyl)benzoate

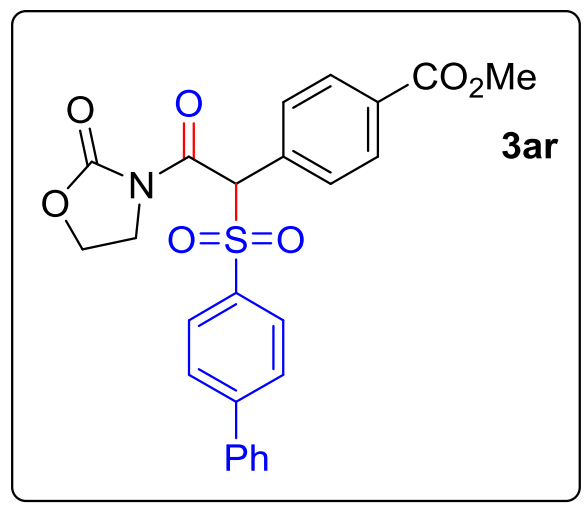

Following the general procedure, 3ar was obtained as a white solid $(17.8 \mathrm{mg}, 0.037 \mathrm{mmol}$, yield: $37 \%) .{ }^{1} \mathrm{H} \mathrm{NMR}\left(400 \mathrm{MHz}, \mathrm{CDCl}_{3}\right) \delta 7.95$ $(\mathrm{d}, J=8.4 \mathrm{~Hz}, 2 \mathrm{H}), 7.64(\mathrm{~s}, 4 \mathrm{H}), 7.60(\mathrm{~d}, J=7.0$ $\mathrm{Hz}, 2 \mathrm{H}), 7.51(\mathrm{~d}, J=8.5 \mathrm{~Hz}, 1 \mathrm{H}), 7.47$ (d, $J=7.6$ $\mathrm{Hz}, 3 \mathrm{H}), 7.38$ (d, $J=8.3 \mathrm{~Hz}, 1 \mathrm{H}), 7.01(\mathrm{~s}, 1 \mathrm{H})$, $4.49-4.38(\mathrm{~m}, 2 \mathrm{H}), 4.24-4.12(\mathrm{~m}, 1 \mathrm{H}), 4.08-$

3.98 (m, 1H), 3.91 (s, 3H). ${ }^{13} \mathbf{C}$ NMR (101 MHz, CDCl $): \delta 166.4,163.5,153.0$, 147.2 , 138.8 , 135.0 , 132.4 , 131.1 , 130.3 , 129.8 , 129.6 , 129.1 , 128.8 , 127.4 , 127.2 , $71.1,62.1,52.3,42.8$. HRMS (ESI, m/z): calculated for $\mathrm{C}_{25} \mathrm{H}_{21} \mathrm{NO}_{7} \mathrm{~S}[\mathrm{M}+\mathrm{H}]^{+}$: 480.1117, found: 480.1116 .

(E)-1-(2-oxooxazolidin-3-yl)-2-phenylvinyl 4-methylbenzenesulfonate

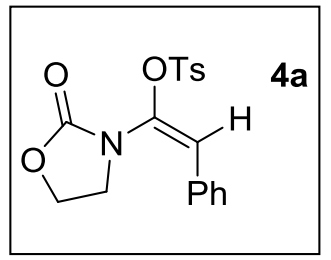

Following the general procedure for the preparation of olefin, $\mathbf{4 a}$ was obtained as a colorless oil $(34.8 \mathrm{mg}, 0.097 \mathrm{mmol}$, yield: 97\%). ${ }^{1} \mathbf{H}$ NMR (400 $\left.\mathbf{M H z}, \mathbf{C D C l}_{3}\right): \delta 7.90(\mathrm{~d}, J=8.3 \mathrm{~Hz}, 2 \mathrm{H})$, $7.38(\mathrm{~d}, J=8.2 \mathrm{~Hz}, 2 \mathrm{H}), 7.32-7.23(\mathrm{~m}, 3 \mathrm{H}), 7.15(\mathrm{~d}, J=8.1 \mathrm{~Hz}$, 2H), $5.97(\mathrm{~s}, 1 \mathrm{H}), 4.36(\mathrm{t}, 2 \mathrm{H}), 3.80(\mathrm{t}, 2 \mathrm{H}), 2.46(\mathrm{~s}, 3 \mathrm{H}) ;{ }^{13} \mathbf{C}$ NMR (101 MHz, CDCl 3$)$ : $\delta 154.6,146.0,136.0,131.7,131.4,130.0,128.8,128.8,128.7,119.5,63.0,43.8$, 21.8. HRMS (ESI, m/z): calculated for $\mathrm{C}_{18} \mathrm{H}_{17} \mathrm{NO}_{5} \mathrm{~S}[\mathrm{M}+\mathrm{H}]^{+}:$360.0906, found: 360.0906.

(E)-1-(2-oxooxazolidin-3-yl)-2-phenylvinyl[1,1'-biphenyl]-4-sulfonate 


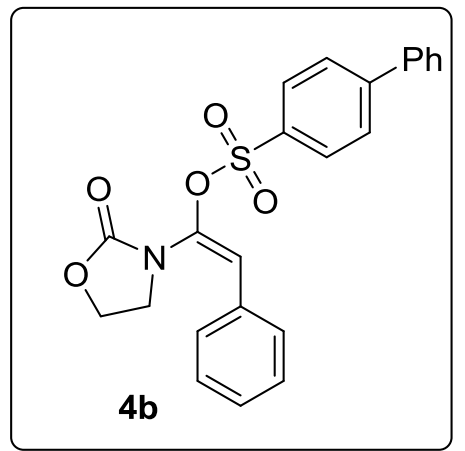

Following the general procedure for the preparation of olefin, $\mathbf{4 b}$ was obtained as a colorless oil $(41.3 \mathrm{mg}, 0.098$ mmol, yield: $98 \%) .{ }^{\mathbf{1}} \mathbf{H}$ NMR (400 $\left.\mathbf{M H z}, \mathbf{C D C l}_{3}\right): \delta 8.09$ $(\mathrm{d}, J=8.6 \mathrm{~Hz}, 2 \mathrm{H}), 7.80(\mathrm{~d}, J=8.6 \mathrm{~Hz}, 2 \mathrm{H}), 7.63(\mathrm{~d}, J=$ $6.9 \mathrm{~Hz}, 2 \mathrm{H}), 7.47(\mathrm{dt}, J=14.9,7.1 \mathrm{~Hz}, 3 \mathrm{H}), 7.35-7.22$ $(\mathrm{m}, 3 \mathrm{H}), 7.17(\mathrm{~d}, 2 \mathrm{H}), 6.04(\mathrm{~s}, 1 \mathrm{H}), 4.37(\mathrm{t}, 2 \mathrm{H}), 3.83(\mathrm{t}$, 2H); ${ }^{13} \mathbf{C}$ NMR (101 MHz, $\left.\mathbf{C D C l}_{3}\right): \delta 154.51,147.46,138.64,135.95,133.06$, $131.25,129.23,129.09,128.86,128.68,128.60,128.04,127.70,127.32,119.54$, 62.87, 43.76. HRMS (ESI, m/z): calculated for $\mathrm{C}_{23} \mathrm{H}_{19} \mathrm{NO}_{5} \mathrm{~S}[\mathrm{M}+\mathrm{H}]^{+}$: 422.1062, found: 422.1062 .

\section{Methyl (E)-4-(2-(2-oxooxazolidin-3-yl)-2-(tosyloxy)vinyl)benzoate}

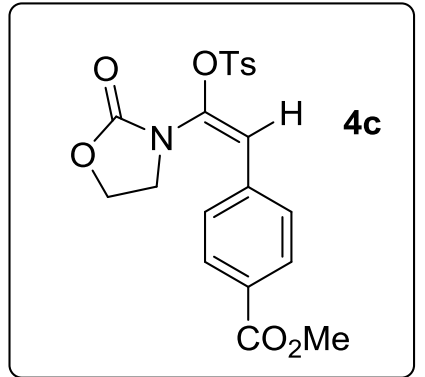

Following the general procedure for the preparation of olefin, 4c was obtained as a colorless oil (40.4 $\mathrm{mg}, 0.097$ mmol, yield: 97\%) ${ }^{\mathbf{1}} \mathbf{H}$ NMR (400 $\left.\mathbf{M H z}, \mathbf{C D C l}_{3}\right): \delta 7.95$ (d, $J=8.4 \mathrm{~Hz}, 2 \mathrm{H}), 7.90(\mathrm{~d}, J=8.3 \mathrm{~Hz}, 2 \mathrm{H}), 7.39(\mathrm{~d}, J=8.2$ $\mathrm{Hz}, 2 \mathrm{H}), 7.21(\mathrm{~d}, J=8.4 \mathrm{~Hz}, 2 \mathrm{H}), 5.97(\mathrm{~s}, 1 \mathrm{H}), 4.41-4.37$

(m, 2H), $3.88(\mathrm{~s}, 3 \mathrm{H}), 3.87-3.82(\mathrm{~m}, 2 \mathrm{H}), 2.47$ (s, 3H); ${ }^{\mathbf{1 3}} \mathbf{C}$ NMR (101 MHz, CDCl 3$)$ : $\delta 166.34,154.12,146.13,137.29,136.21,131.45,129.92,129.85,129.76,128.76$, $127.92,117.86,62.85,52.13,43.78,21.75$. HRMS (ESI, m/z): calculated for $\mathrm{C}_{20} \mathrm{H}_{19} \mathrm{NO}_{7} \mathrm{~S}[\mathrm{M}+\mathrm{H}]^{+}:$418.0960, found: 418.0960. 


\section{References}

[1] Wang, J.; Gao, Y.; Wu, G.; Zhou, Q. Angew. Chem. Int. Ed. 2018, 57, 2716-2720.

[2] Arsenyan, P.; Paegle, E.; Belyakov, S.; Kirsh, G. Tetrahedron Lett. 2015, 56, 4554-4557.

[3] Martin, N.; Cremonesi, A.; Davies, P. W. Chem. Commun. 2011, 47, 379-381.

[4] Stahl,S.; Hamada T.; Ye, X. J. Am. Chem. Soc. 2008, 130, 833-835.

[5] Dumitrescu, L.; Davies, P. W.; Cremonesi, A. Angew. Chem. Int. Ed. 2011, 50, 8931-8935.

[6] Maulide, N.; Pinto, A.; Kaiser, D.; Maryasin, B.; Mauro, G. D.; González, L. Chem. Eur. J. 2018, $24,2515-2519$.

[7] Maulide, N.; Xie, L-G.; Niyomchon, S.; Mota, A. J.; González, L. Nat. Commun, 2016, 7, 10914.

[8] Alcaide, B.; Almendro, P.; Lázaro-Milla, C. Chem. Eur. J. 2016, 22, 8998-9005.

[9] Zhu, H.; Huang, H.; He, G.; Zhu, X.; Qiu, S.; J. Org. Chem. 2015, 80, 3480-3487.

[10] Zhao, J.; Tu, Y.; Zeng, X, Wang, H. Org. Lett. 2018, 20, 280-283.

[11] Knowles, R. R.; Choi, G. J.; Zhu, Q.; Miller, D. C.; Gu, C. J. Nature 2016, 539, 268-271

[12] D. C. Harris, Quantitative Chemical Analysis, Freeman, 9th edn, 2015.

[13] Cismesia, M. A.; Yoon, T. P. Chem. Sci. 2015, 6, 5426.

[14] Hatchard; C. G.; Parker, C. A. Proc. Roy. Soc. (London) 1956, A23, 518.

[15] Kuhn, H. J.; Braslavsky, S. E.; Schmidt, R. Pure Appl. Chem. 2004, 76, 2105.

[16] Monalti, M., et. al. Chemical Actinometry. Handbook of Photochemistry, 3rd Ed; Taylor \& Francis Group, LLC. Boca Raton, FL, 2006, 601.

[17] Demas, J. N.; Bowman, W. D.; Zalewskl, E. F.; Velapoidl, R. A. J. Phys. Chem. 1981, 85, 2766.

[18] Singh, A.; Teegardin, K.; Kelly, M.; Prasad, K. S.; Krishnan, S.; Weave, J. D. J. Organomet. Chem. 2015, 776, 51.

[19] Frisch, M. J.; Trucks, G. W.; Schlegel, H. B.; Scuseria, G. E. Gaussian, Inc., Wallingford CT, 2016. 
6. ${ }^{1} \mathrm{H}$ and ${ }^{13} \mathrm{C}$ NMR spectra of structurally novel compounds
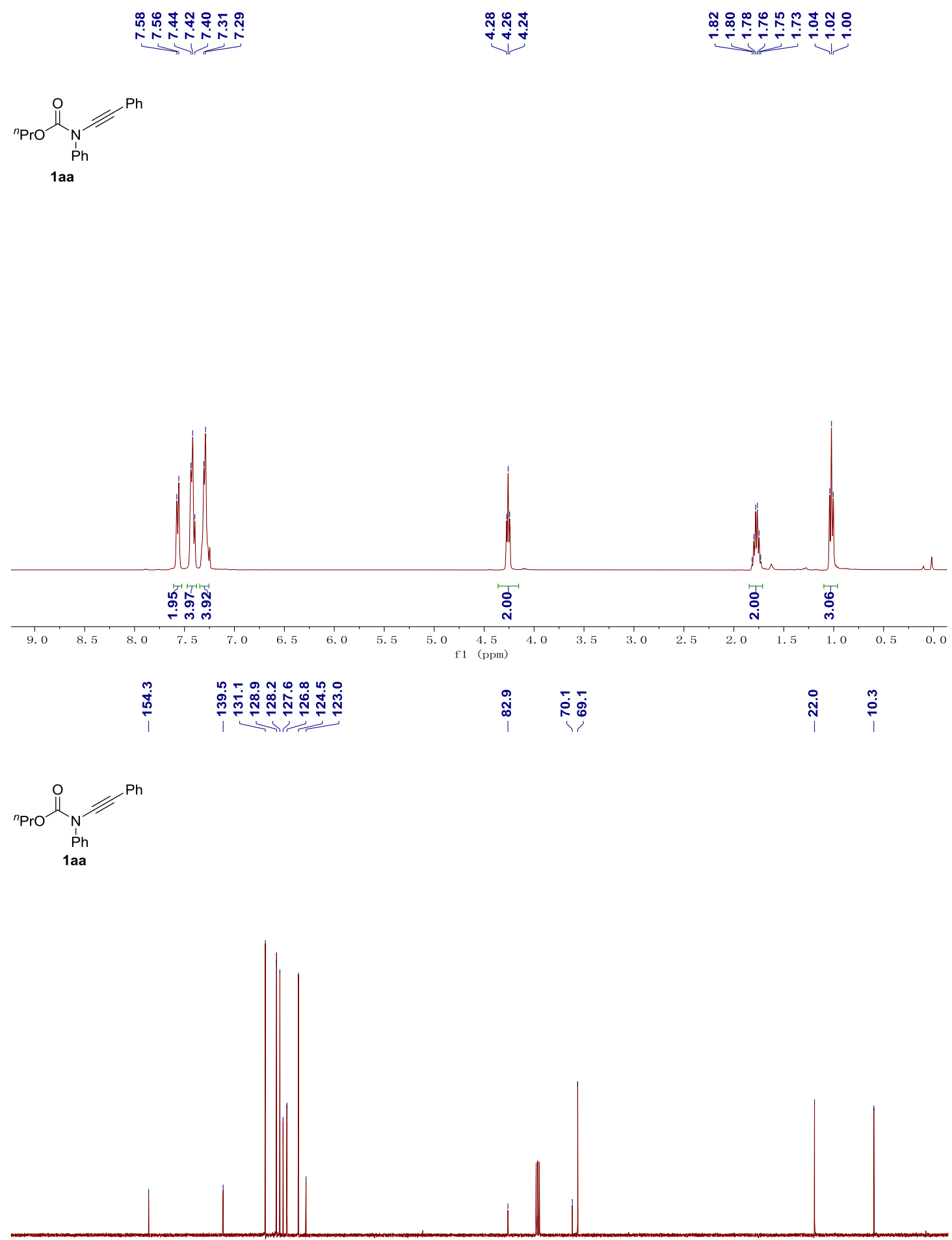

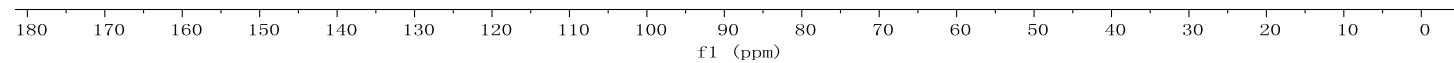




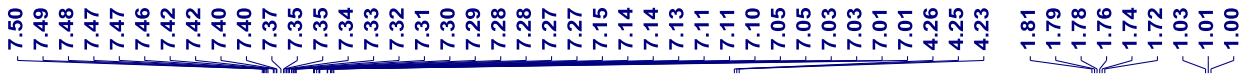
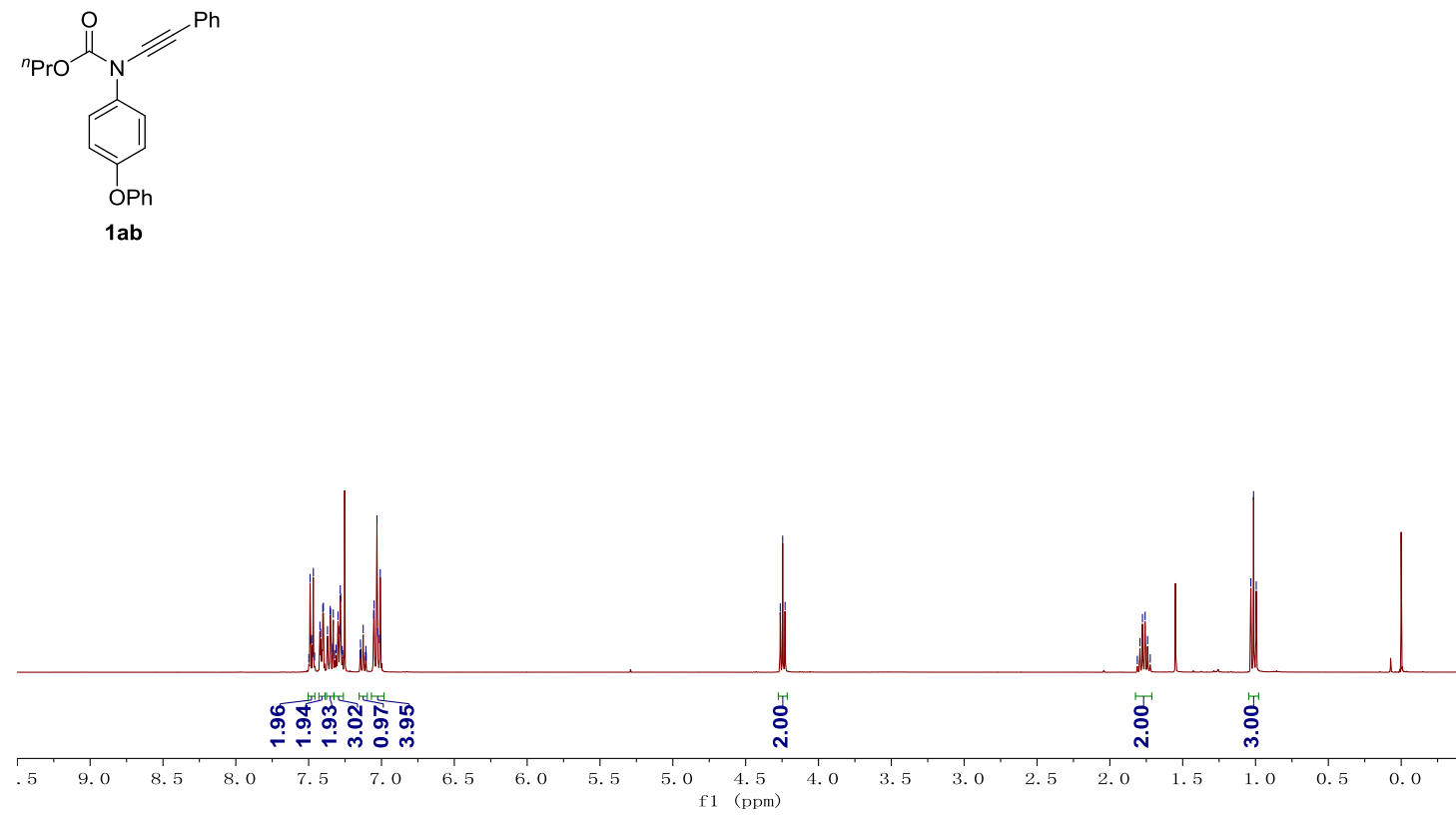

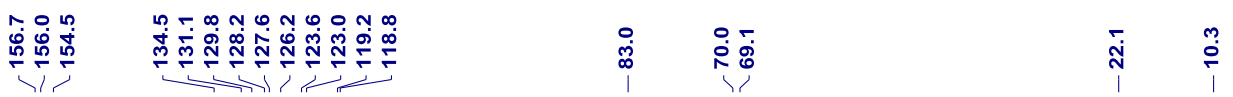
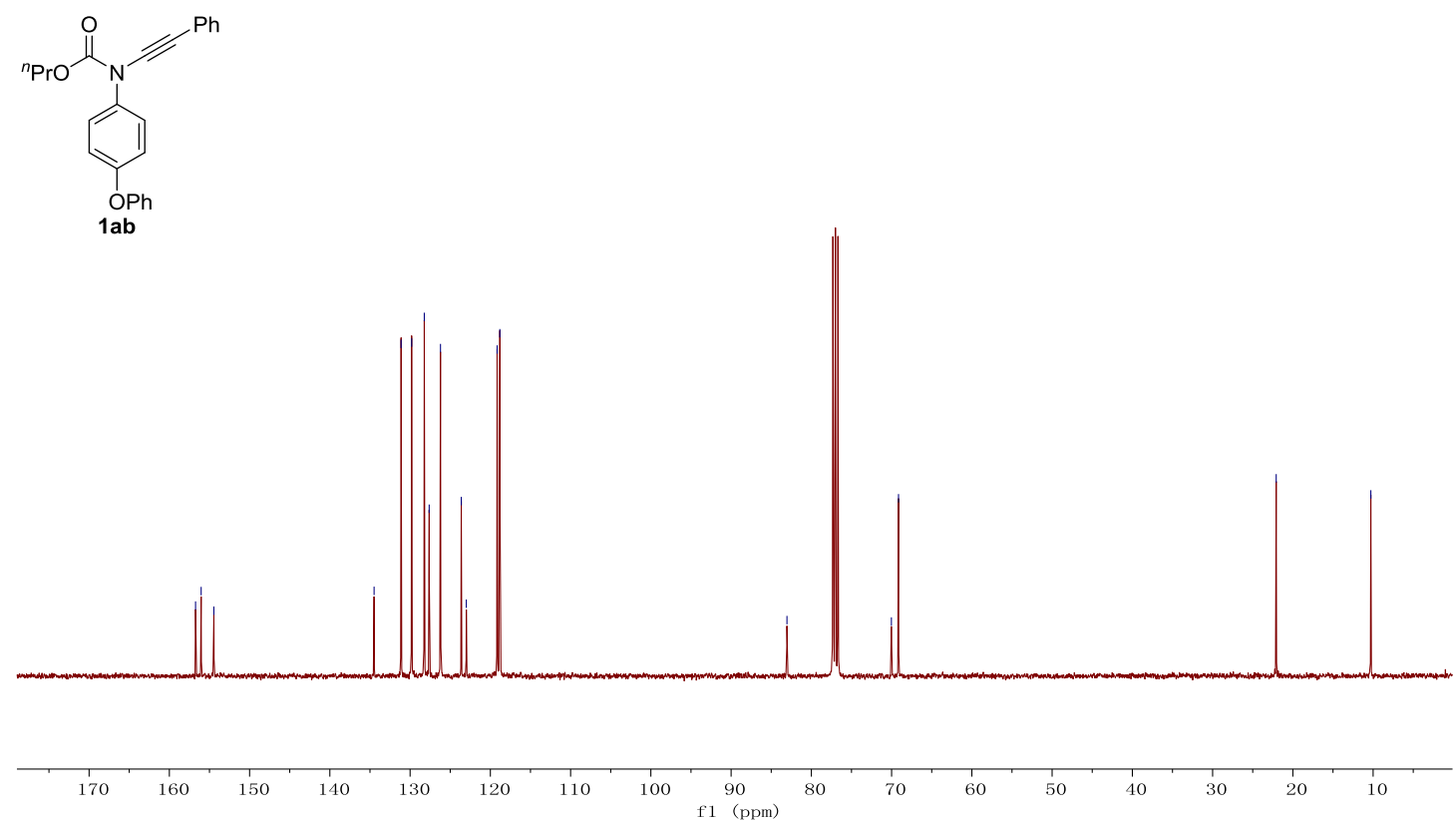


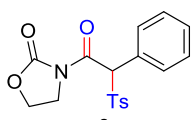

3a

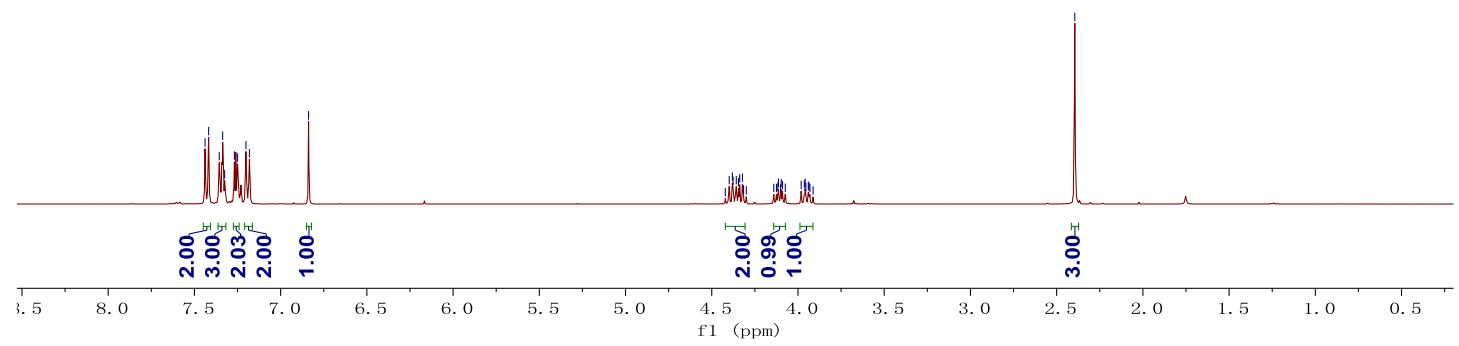

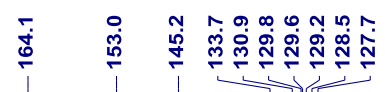

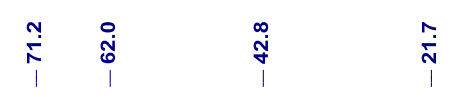

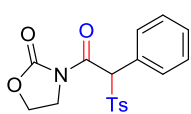

3a

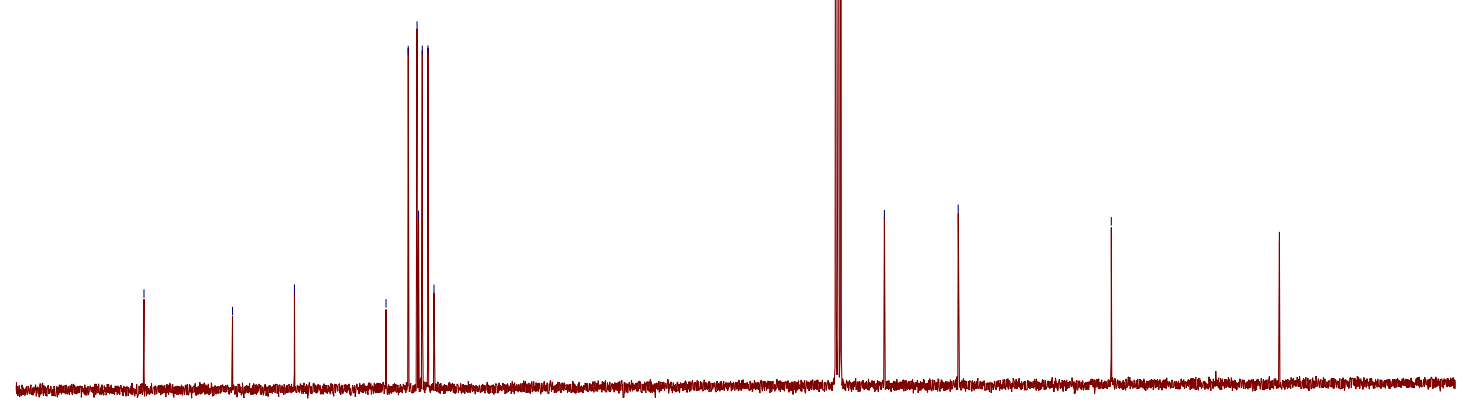

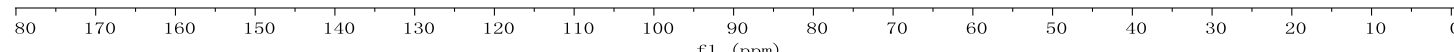



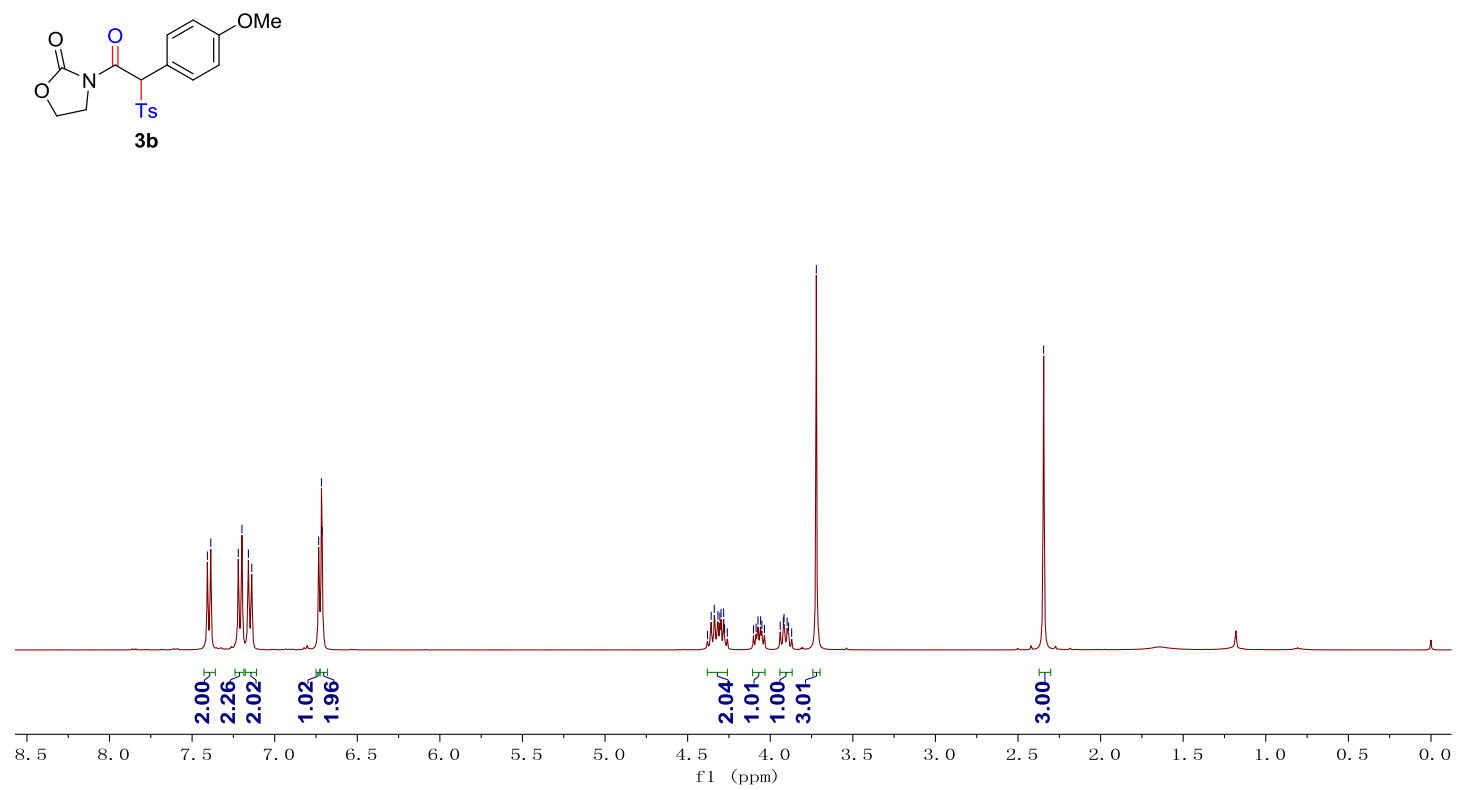

至

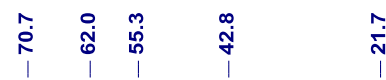

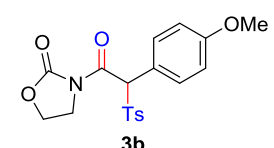

3b
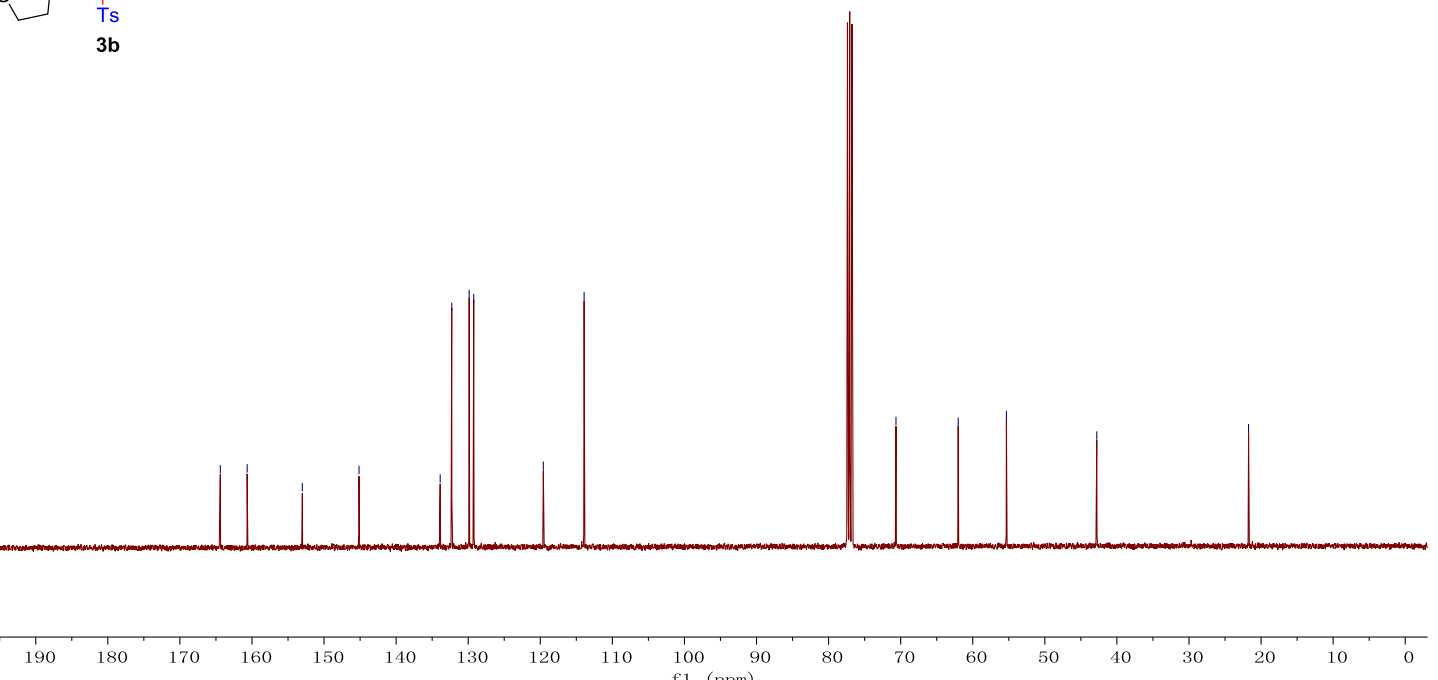

100 (ppm) 

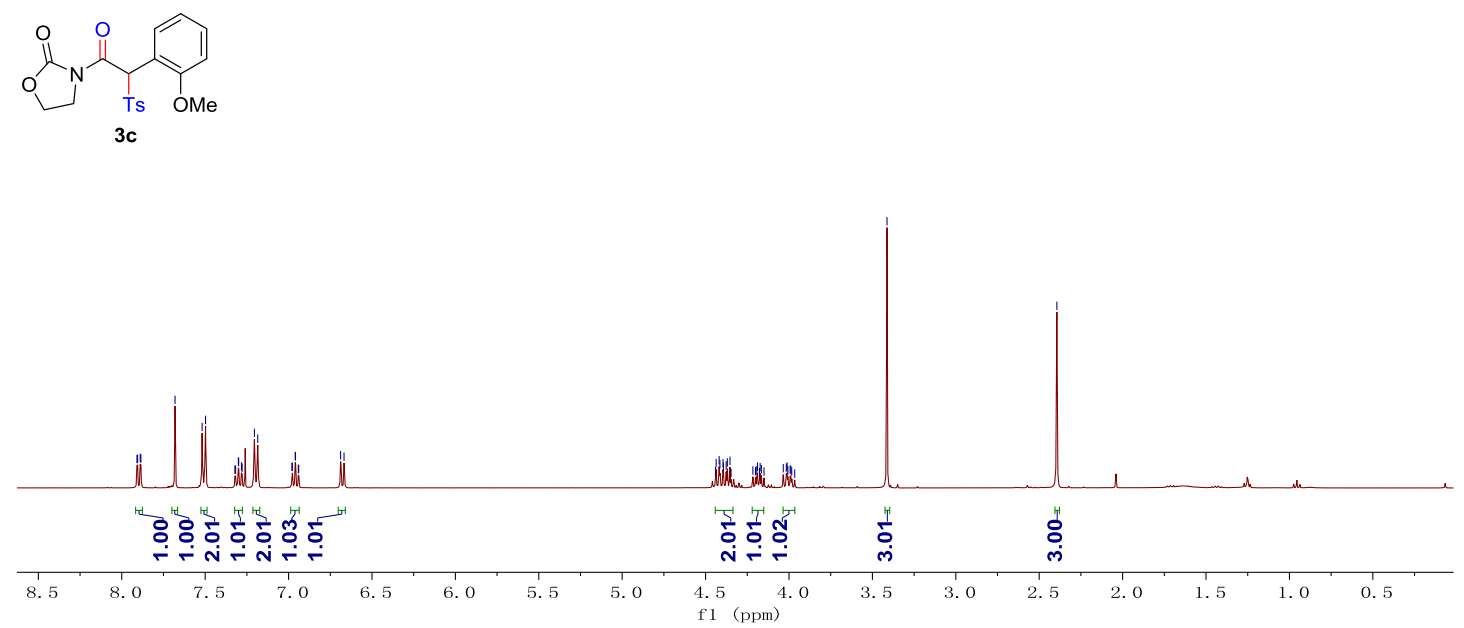

总
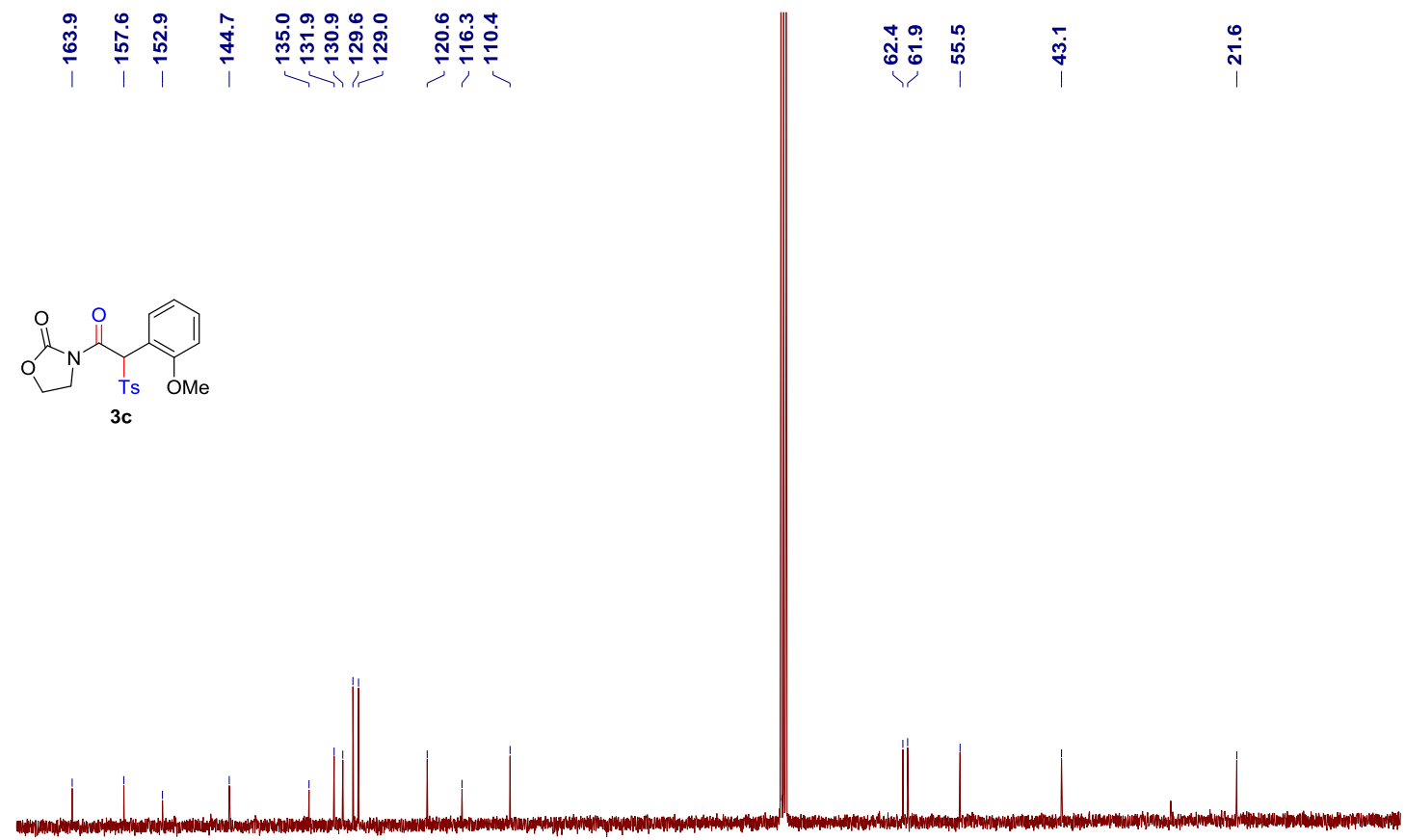

$\stackrel{7}{70}$
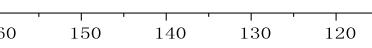

$90\left({ }^{80}\right.$ 


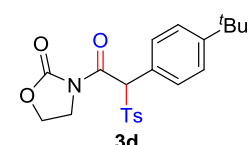

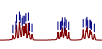

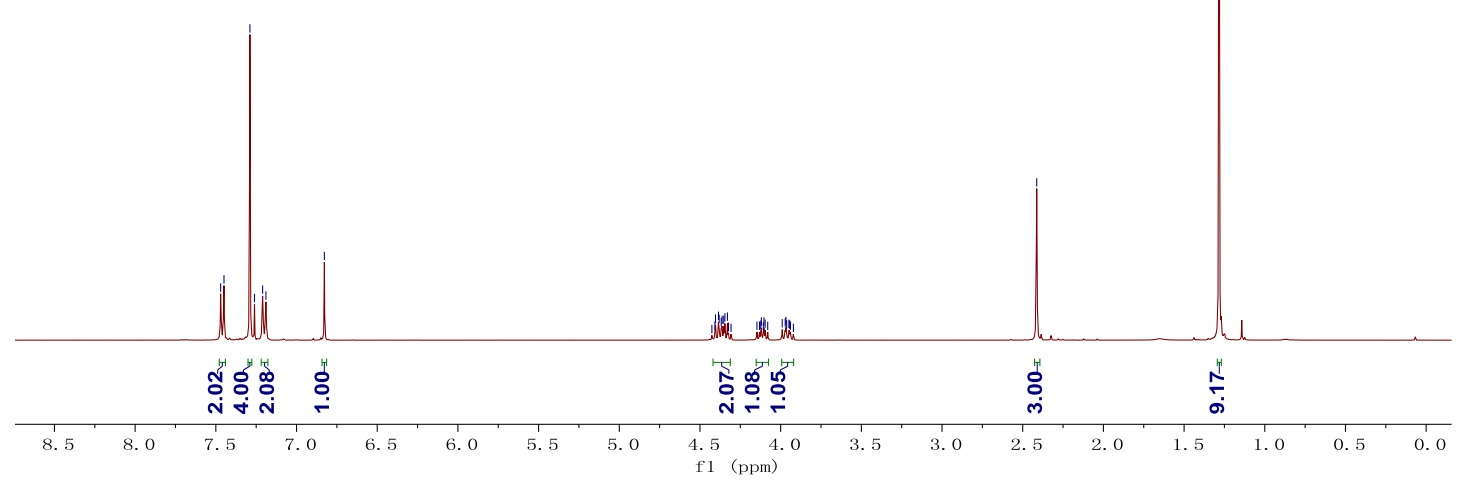

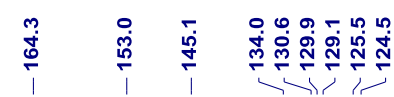

i

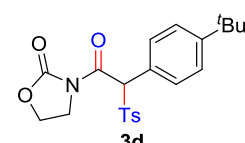

$3 d$

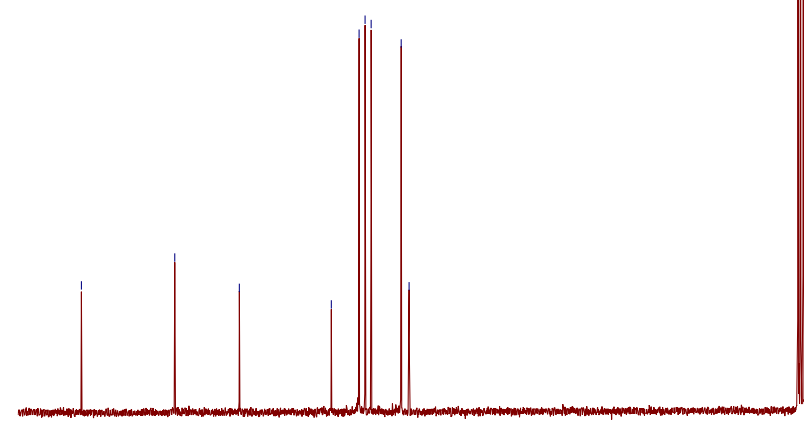




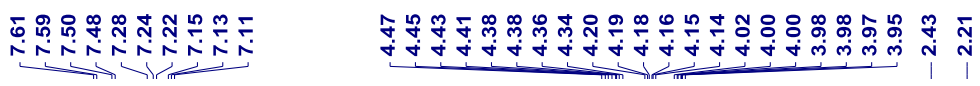
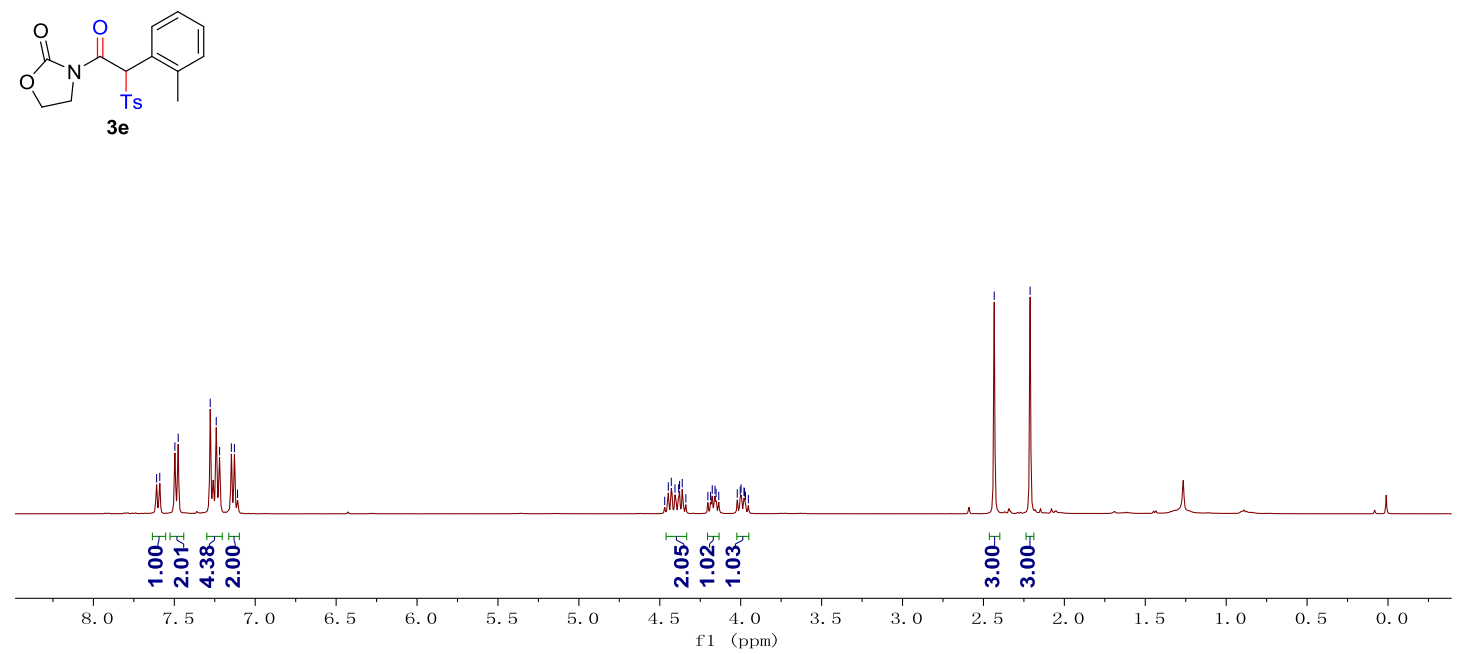

范

@̊ำ

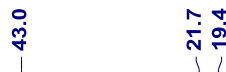

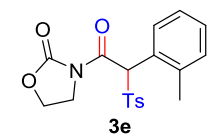

$3 e^{T s}$
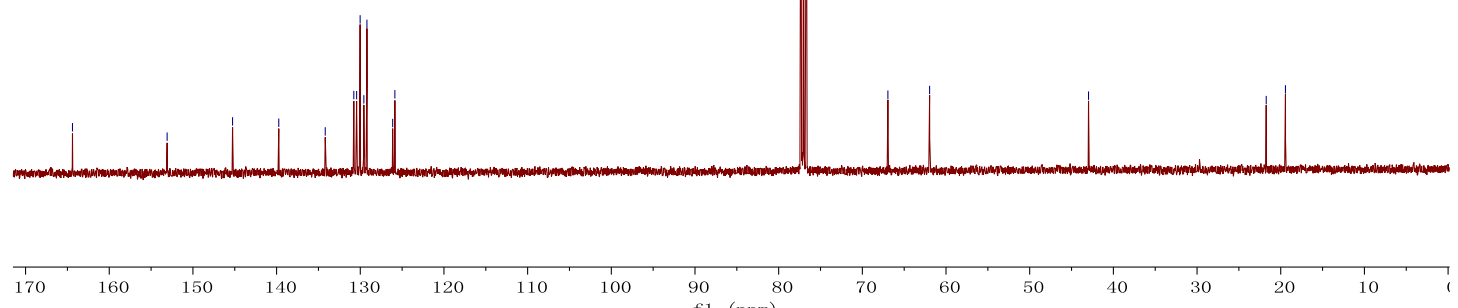

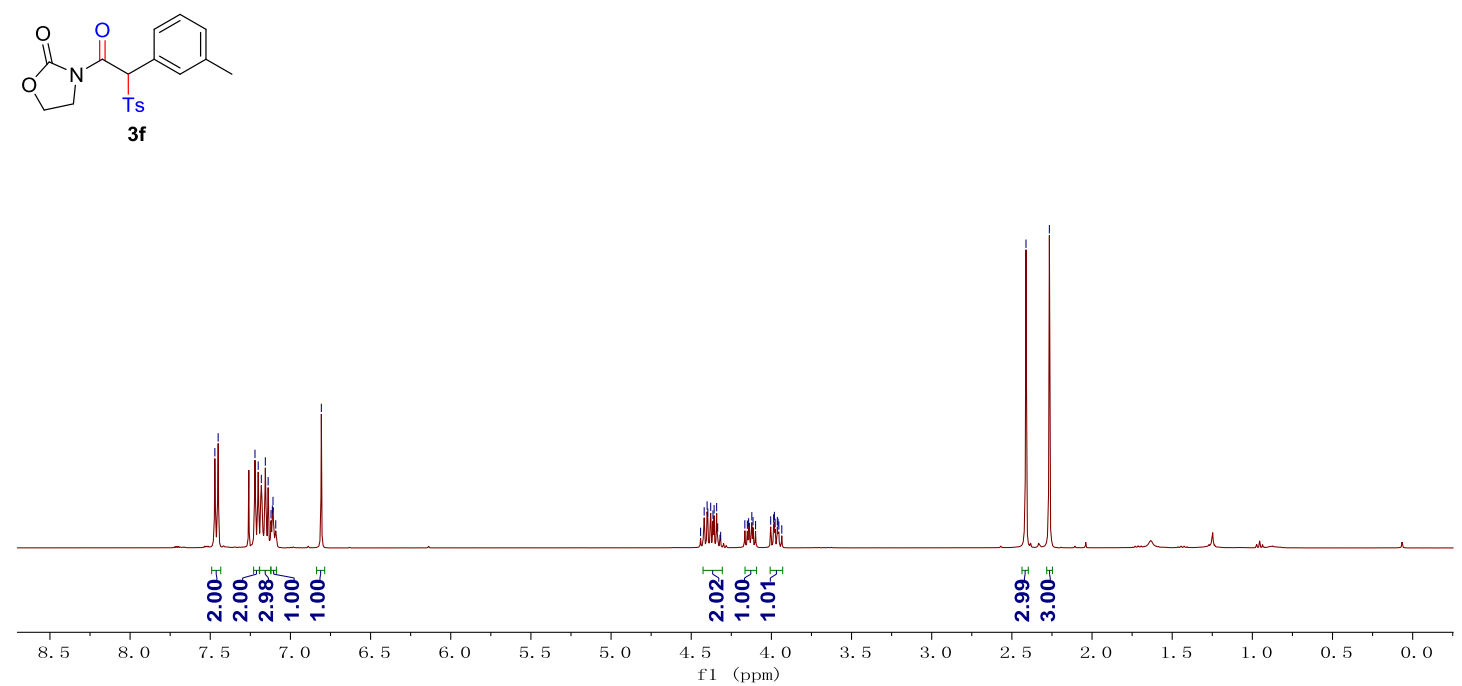

竞

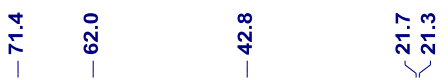
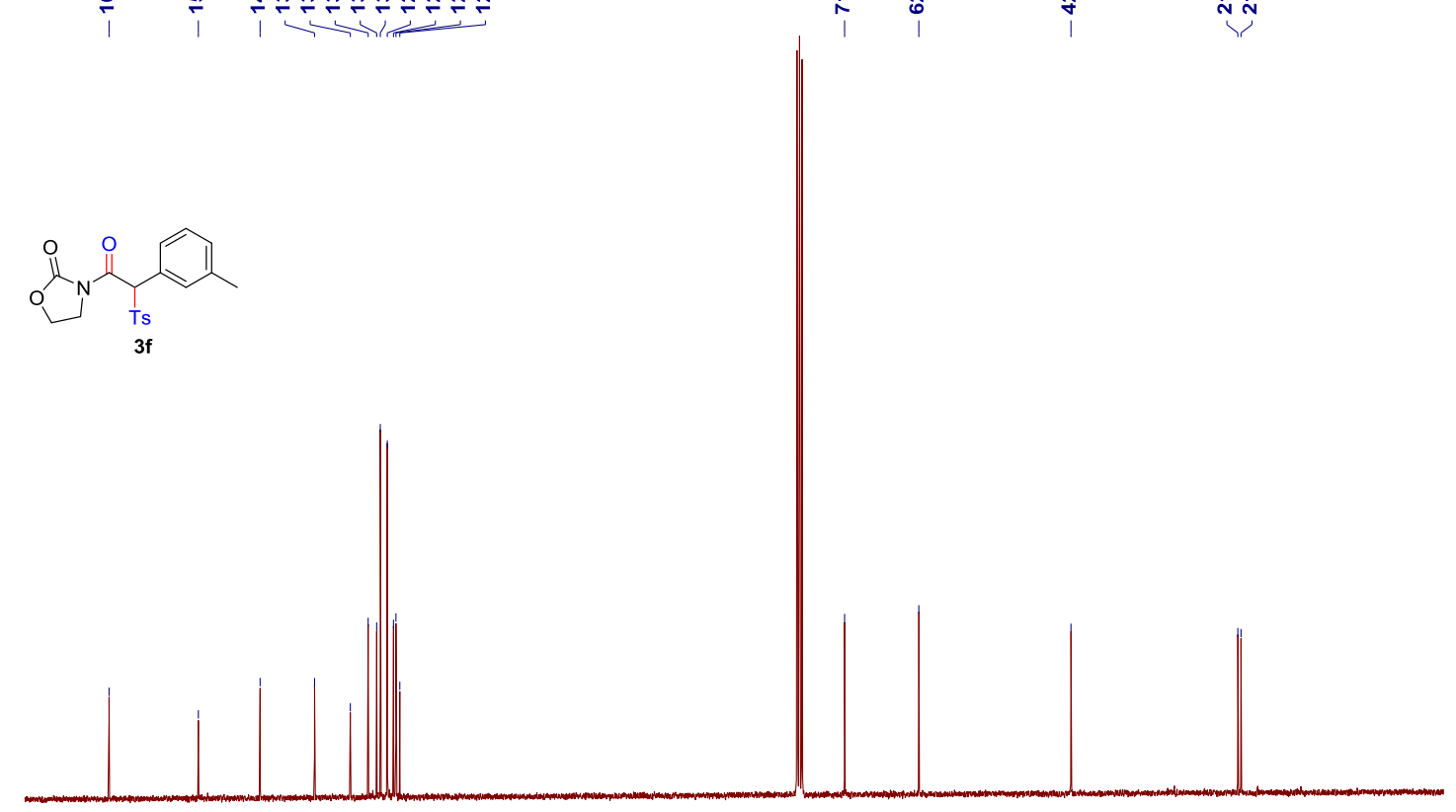

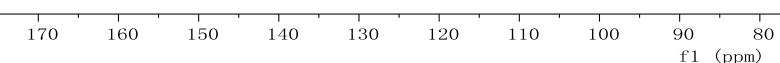




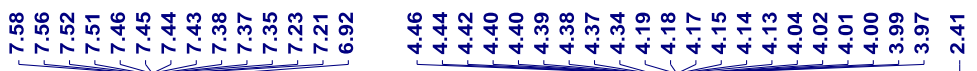

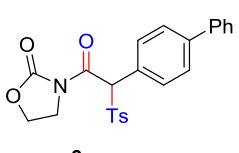

$3 \mathbf{g}$

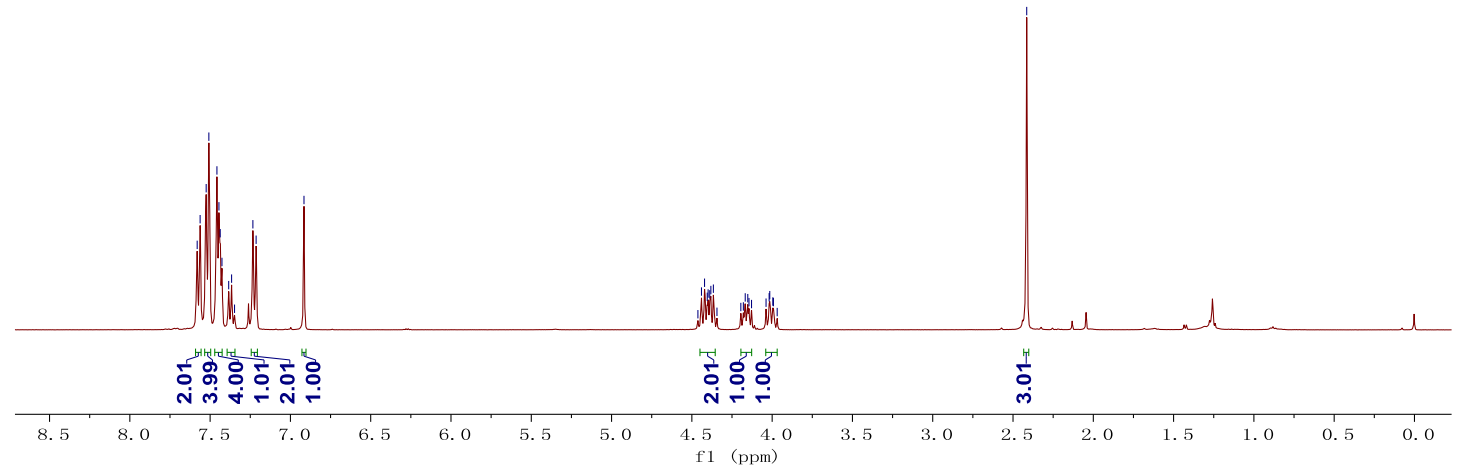

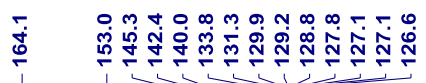

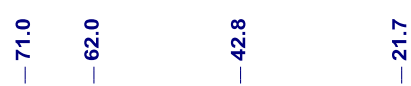

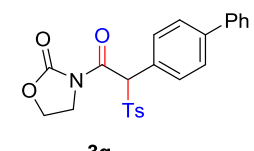

$3 \mathrm{~g}$

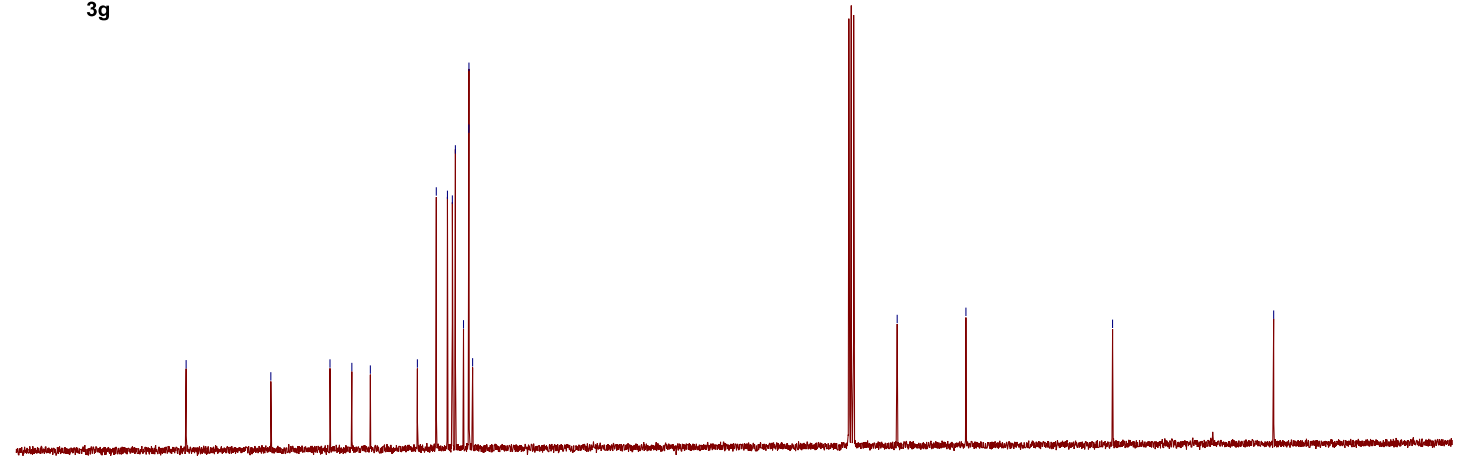

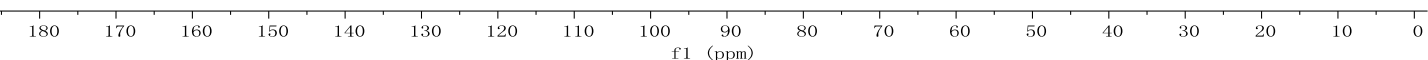



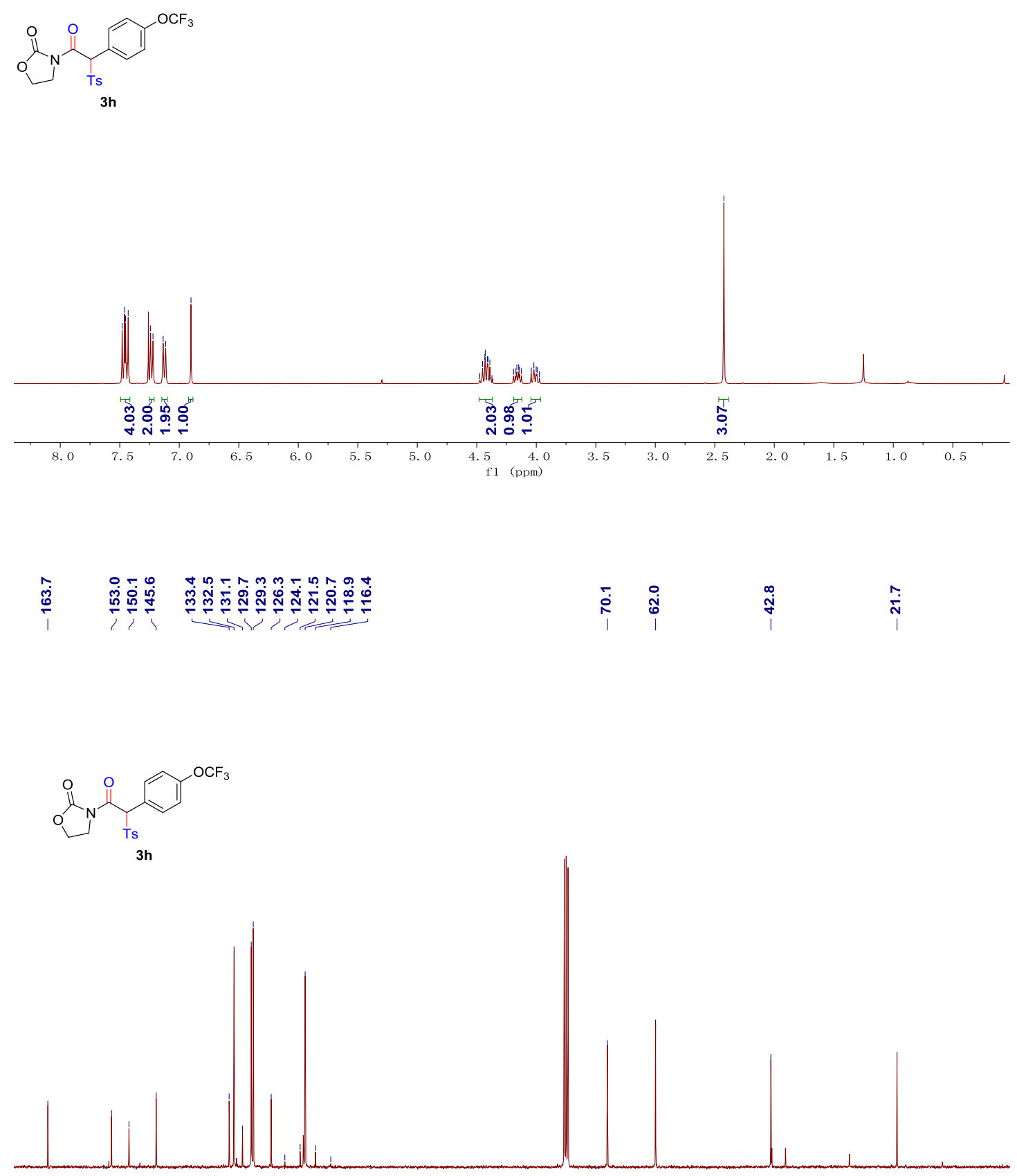

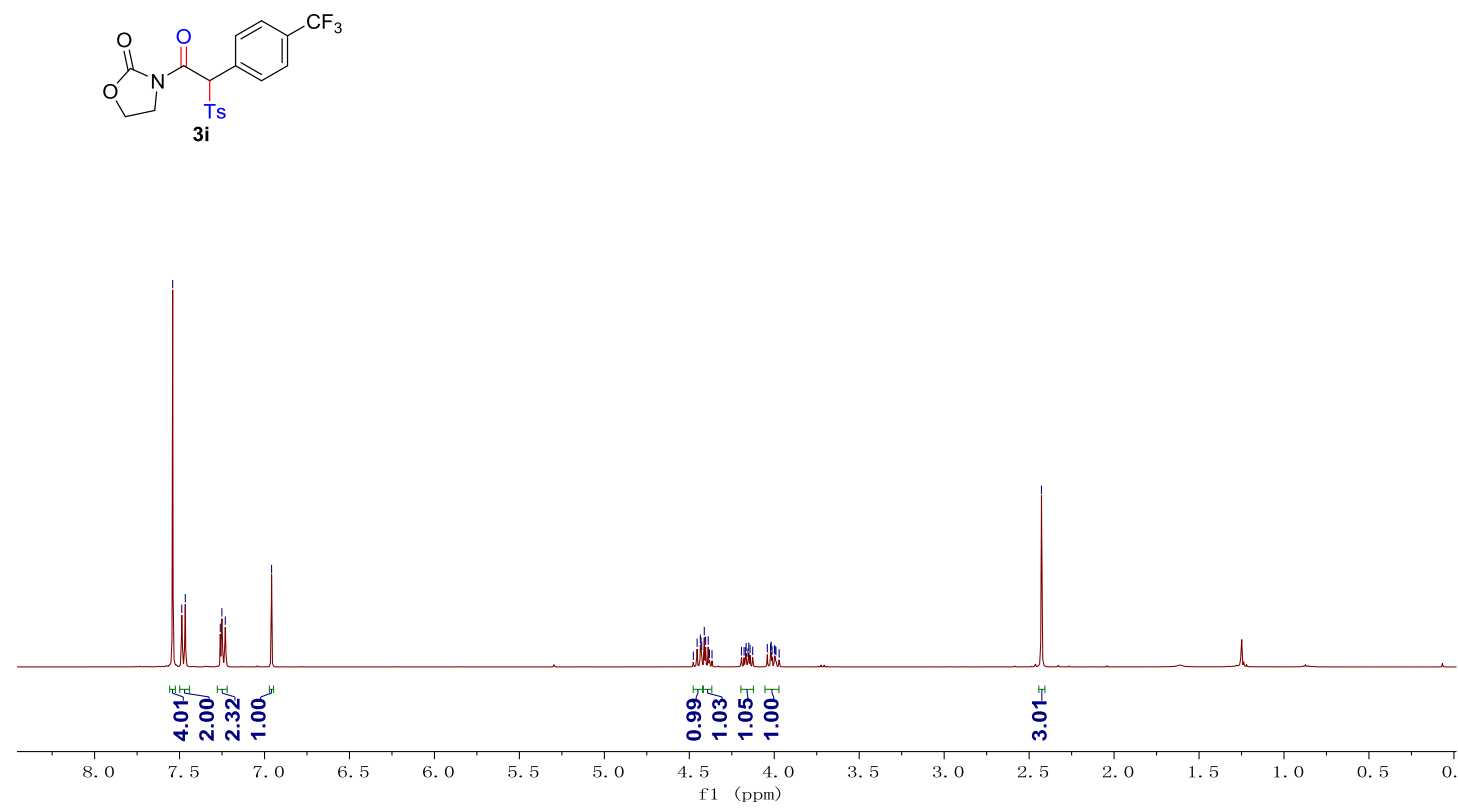

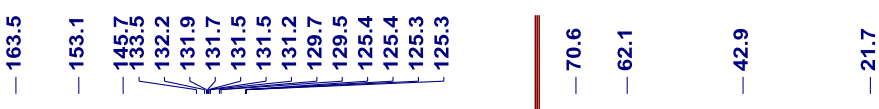

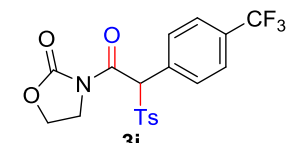

$3 \mathrm{i}$

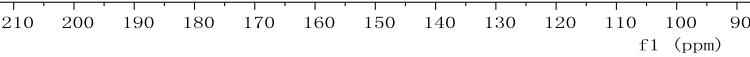


$\sqrt$\[ 幺 \]$$
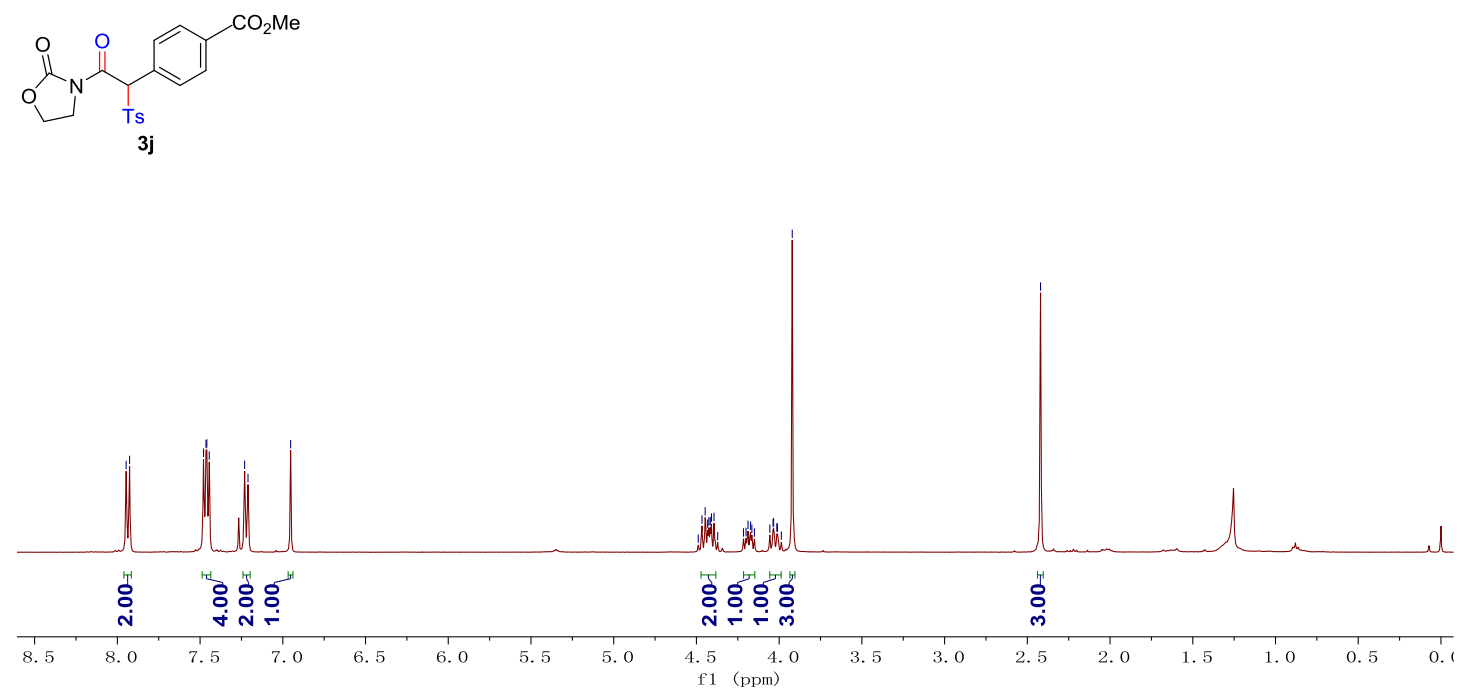

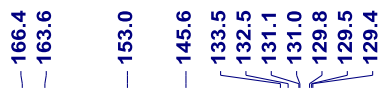

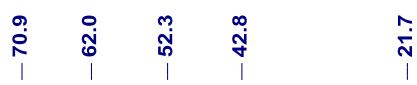
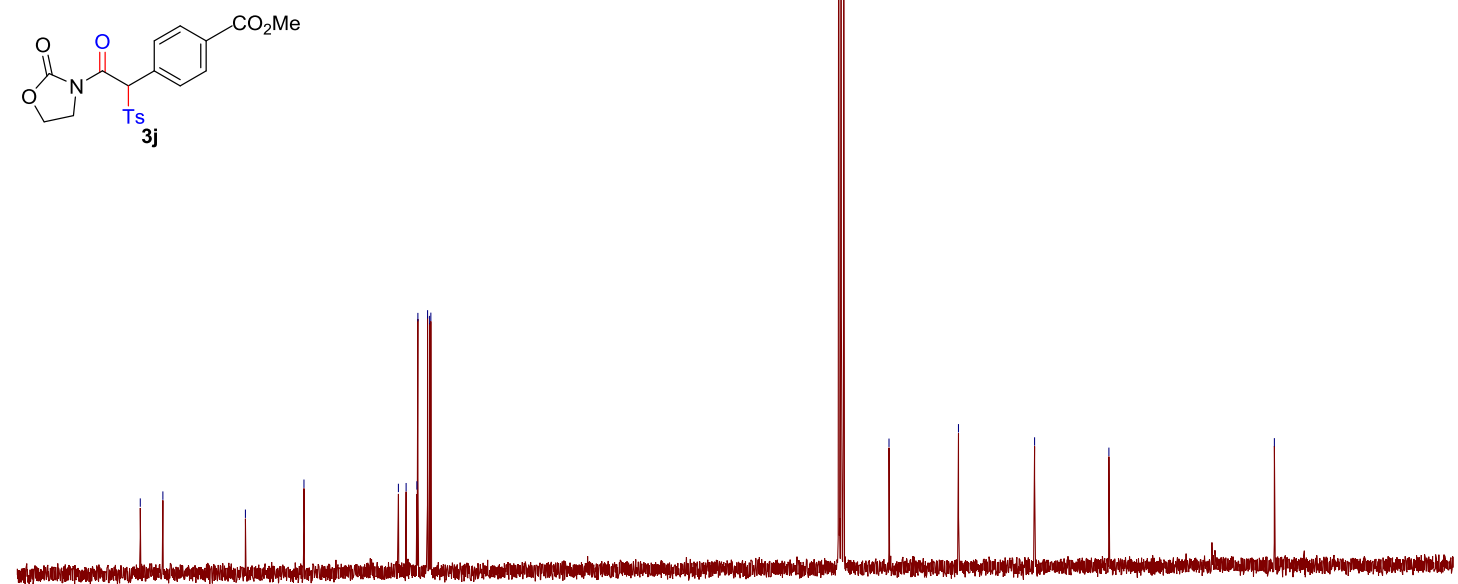

$\begin{array}{lllllllllllllllllll}1 & 1 \\ 180 & 170 & 160 & 150 & 140 & 130 & 120 & 110 & 100 & 90 & 80 & 10 & 60 & 50 & 40 & 30 & 20 & 10 & 0\end{array}$ 

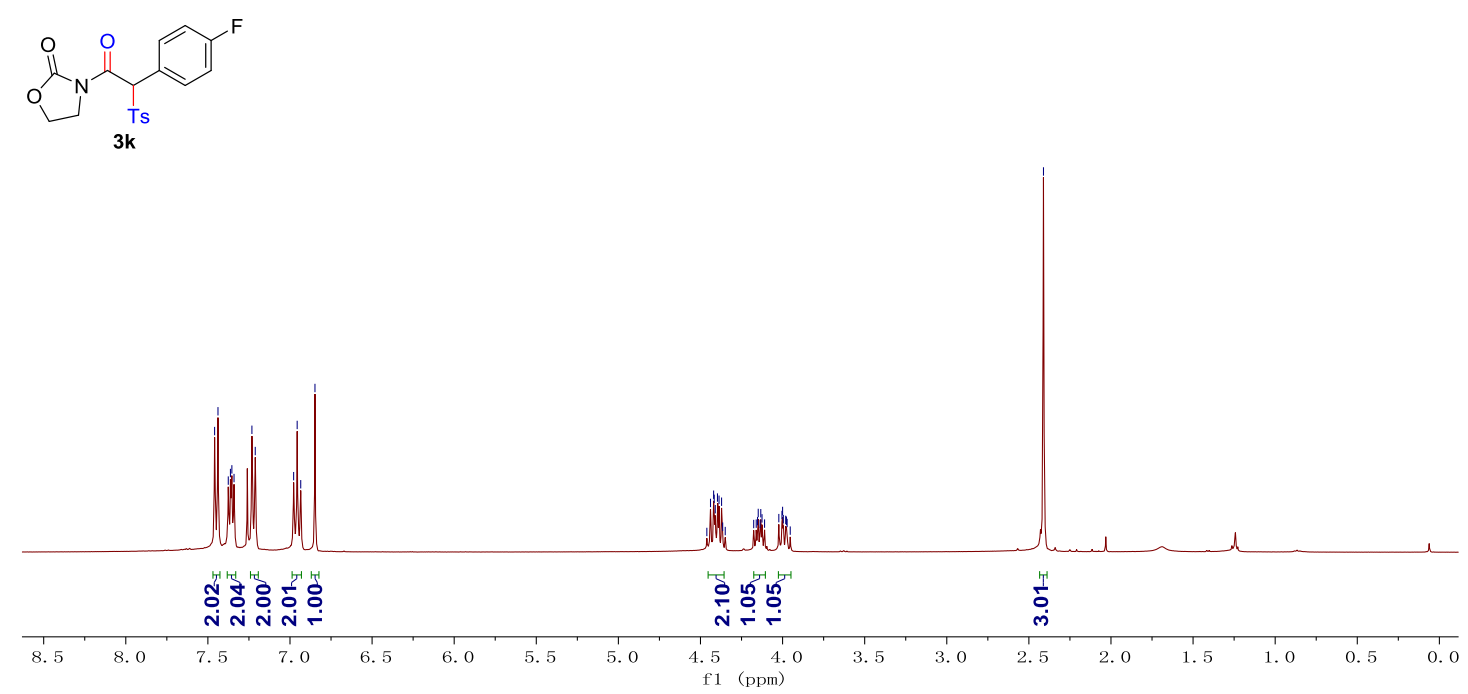

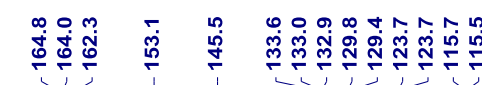

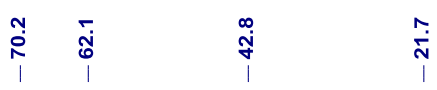

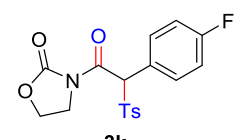

3k

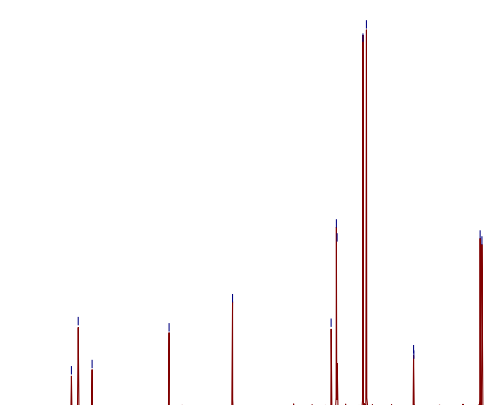

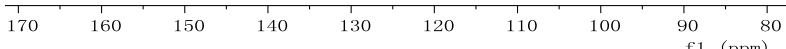



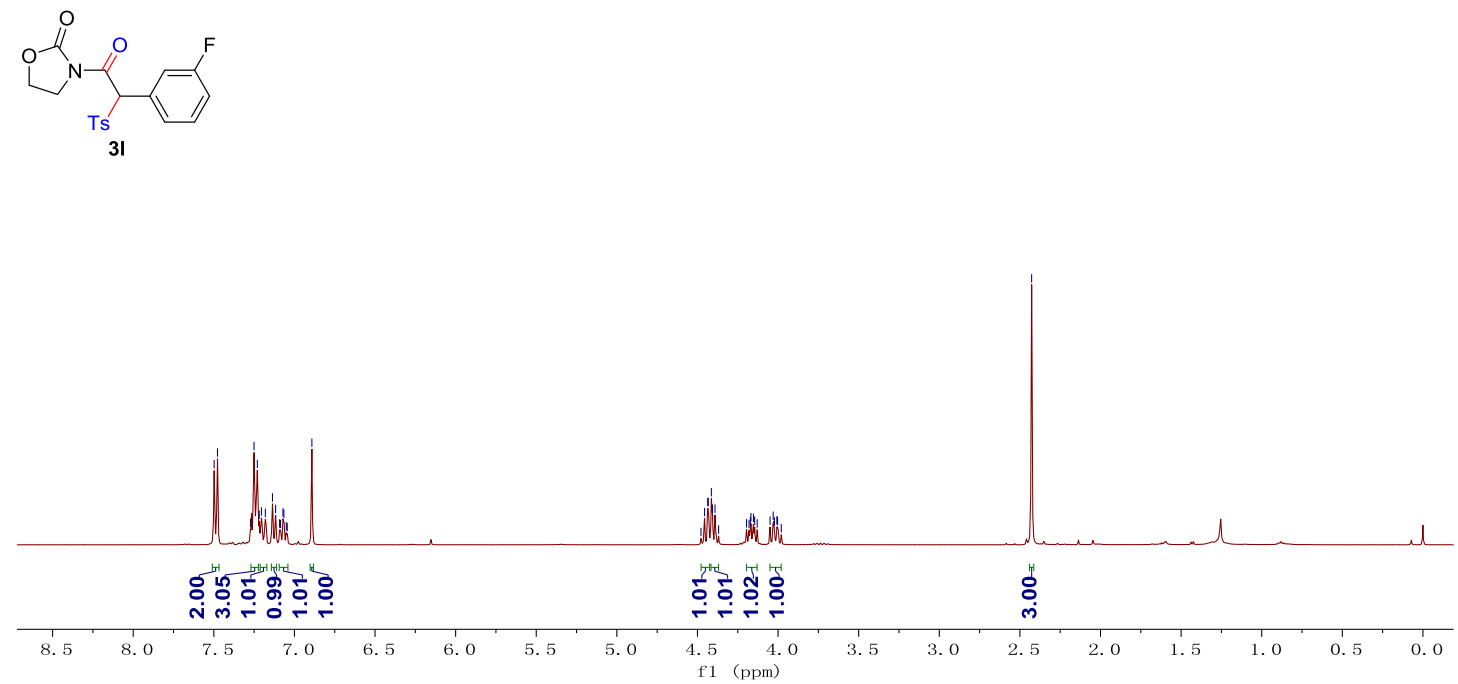

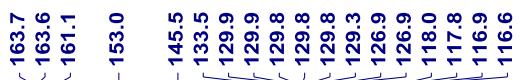

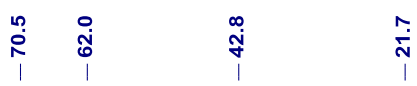
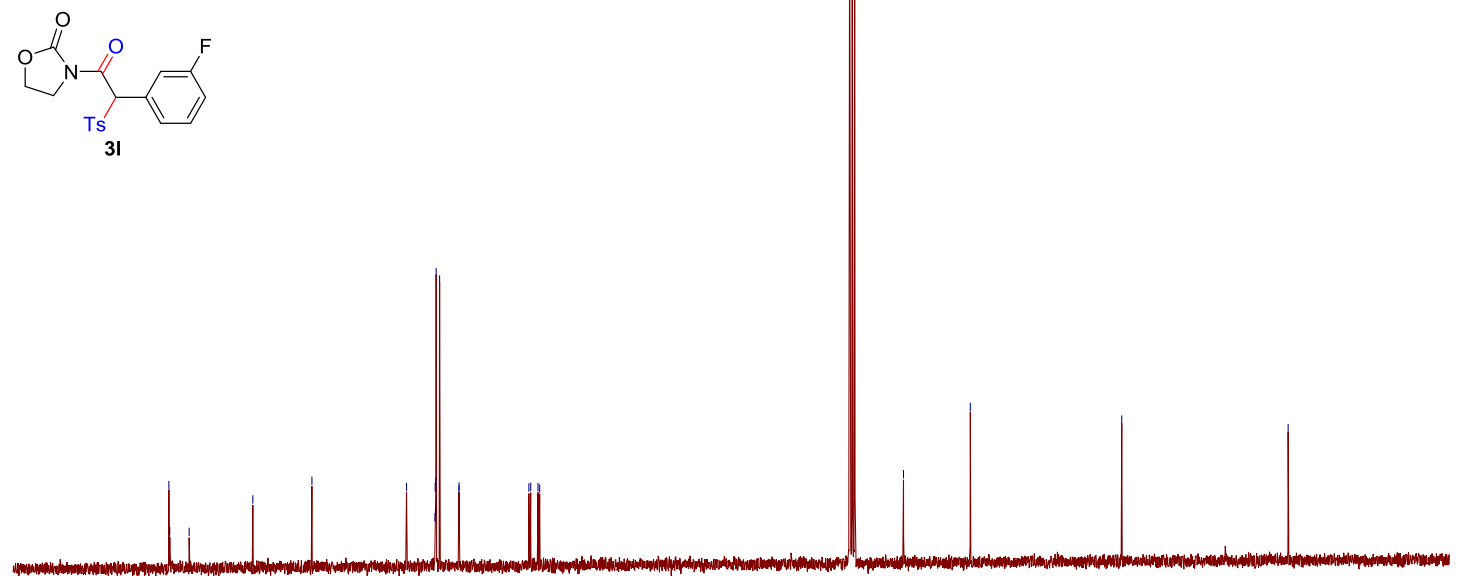

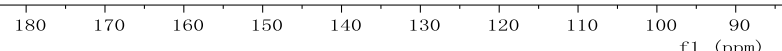




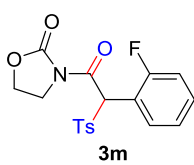

$3 \mathrm{~m}$

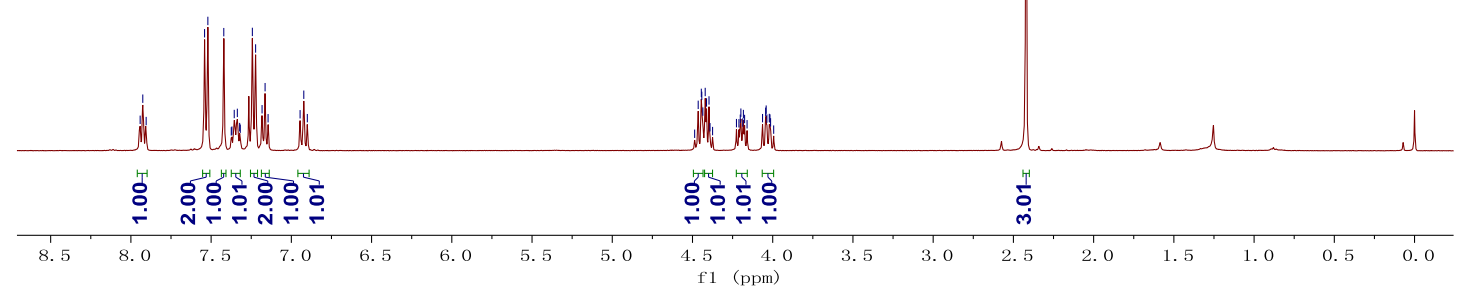

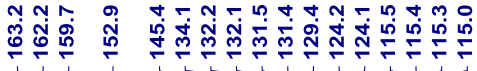

ปู่
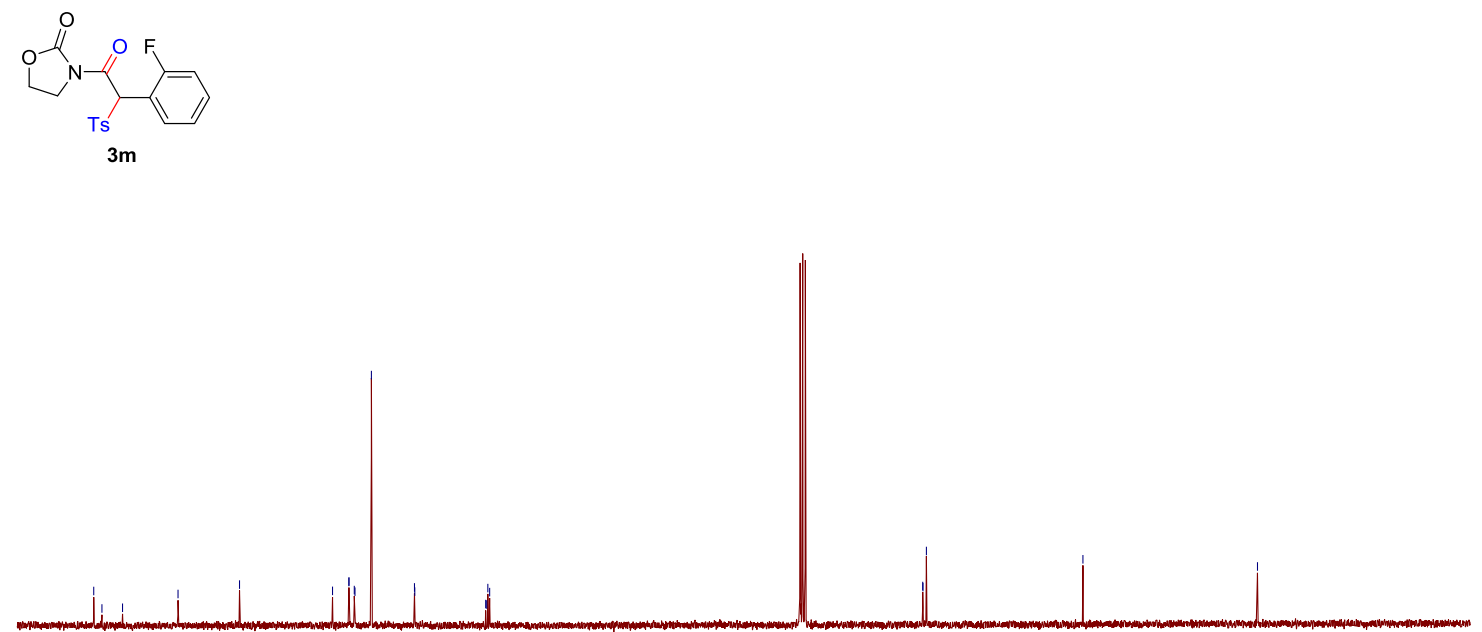

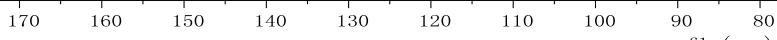
f1 (ppm) 

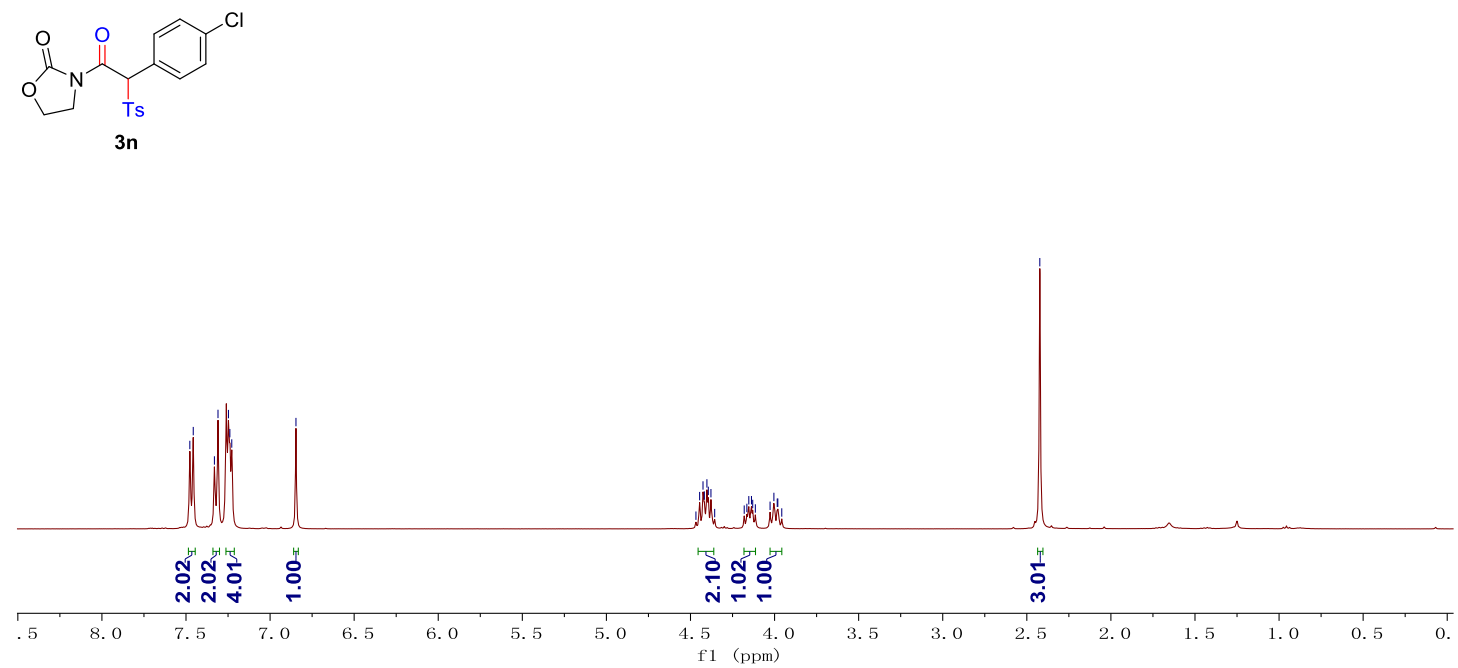

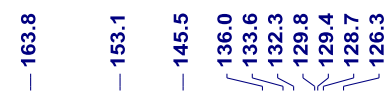

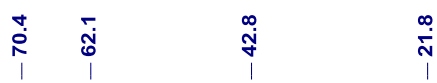
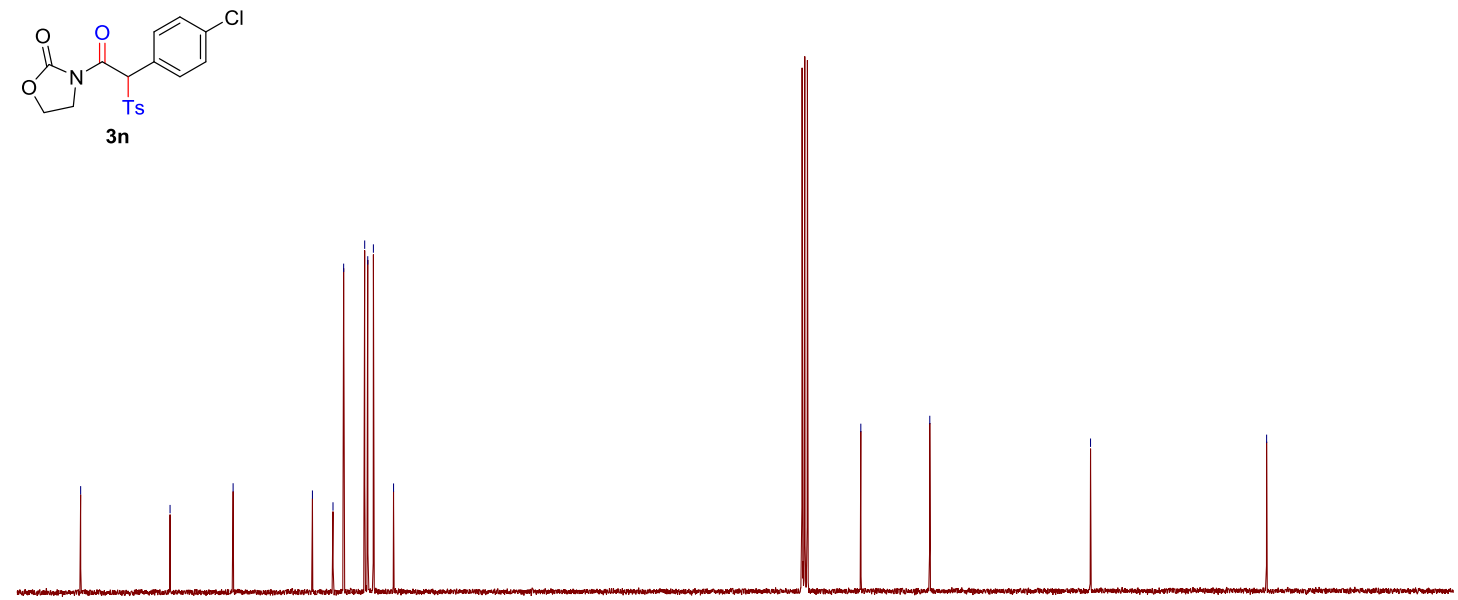

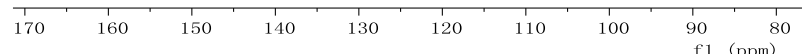



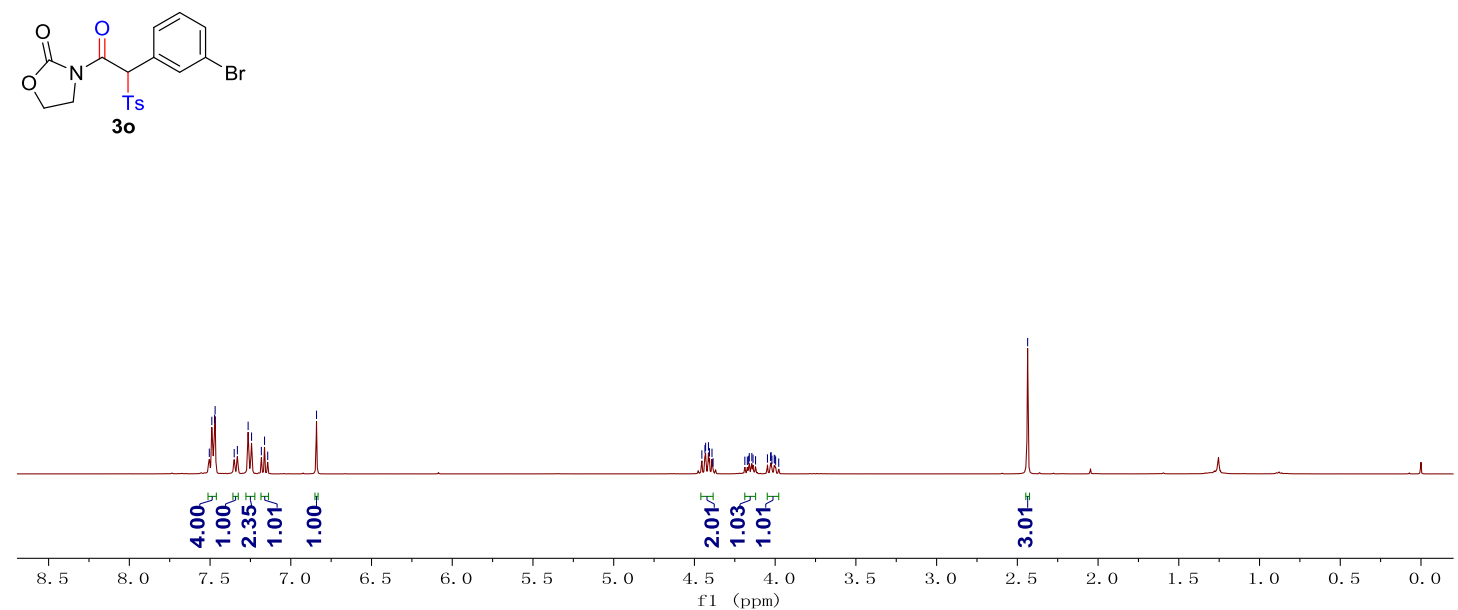

I

คิ่

$\stackrel{\infty}{\stackrel{\infty}{+}}$
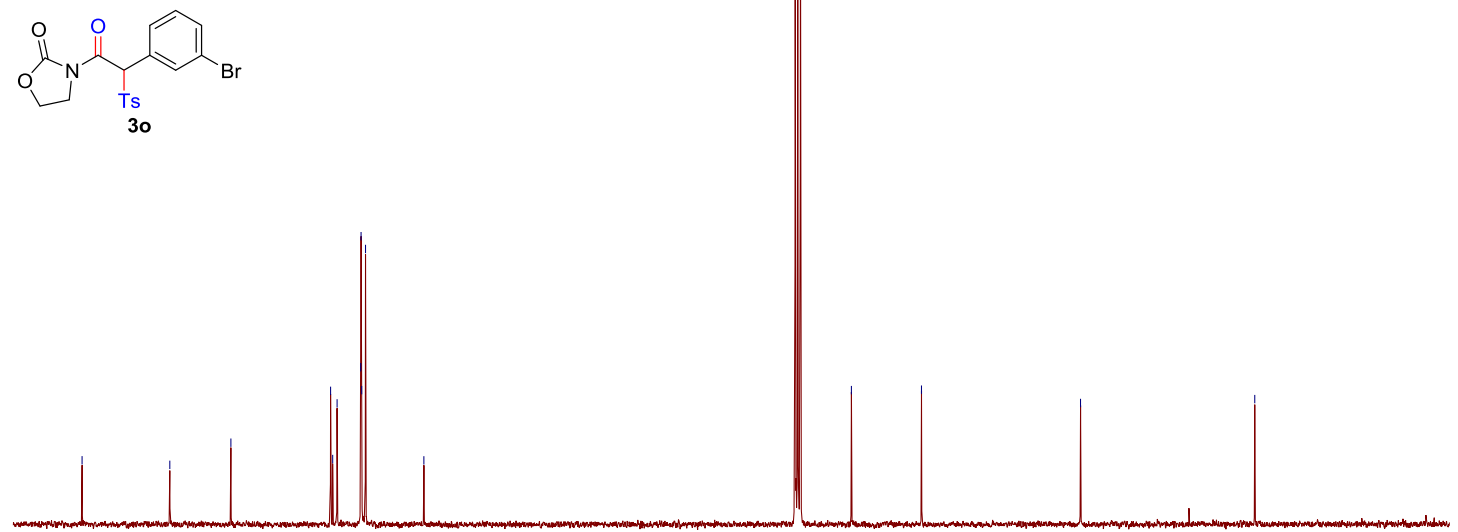

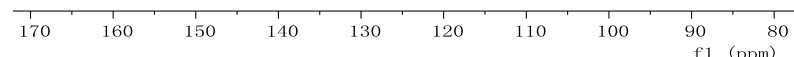



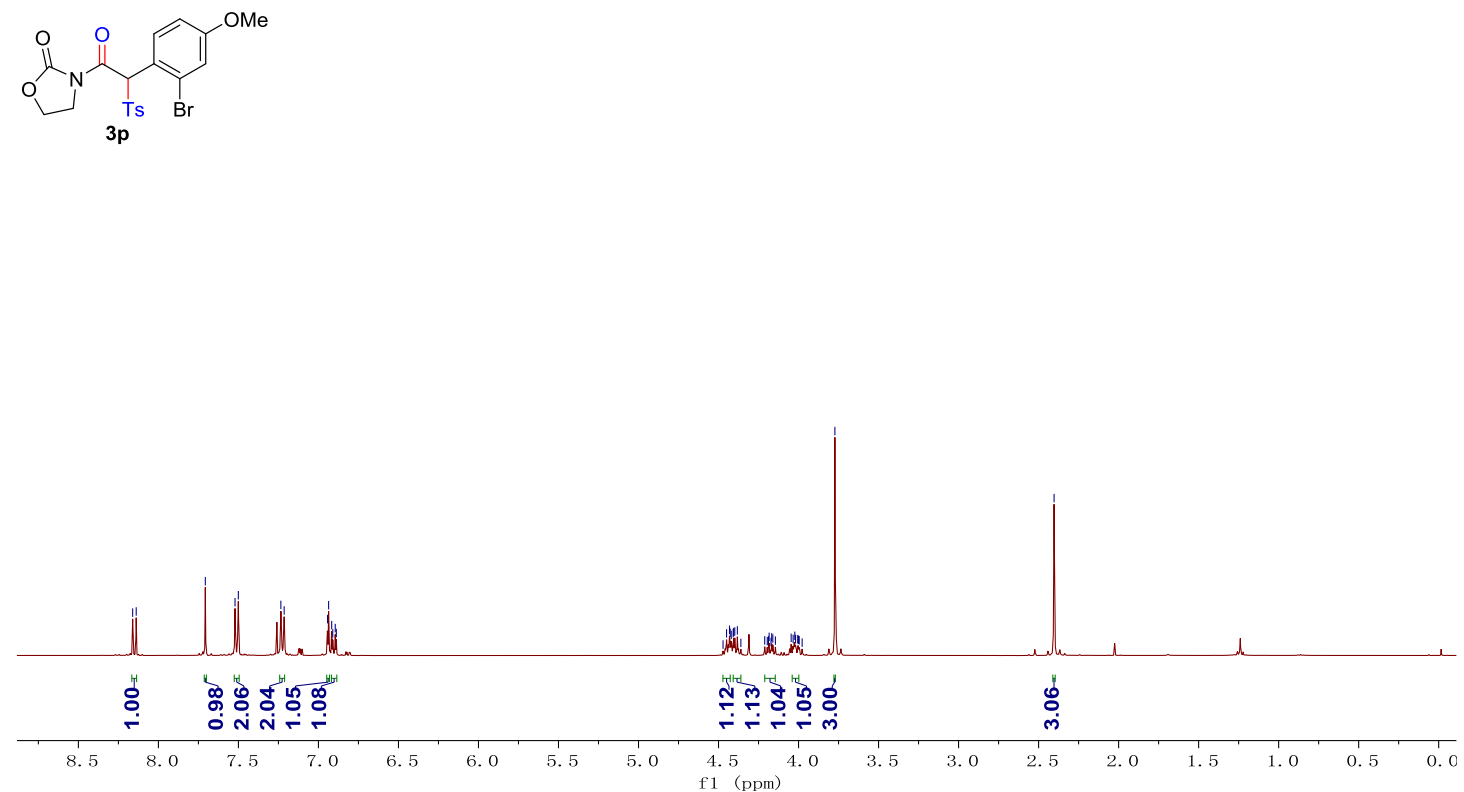

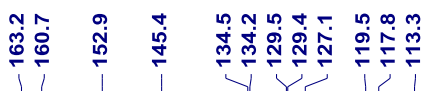

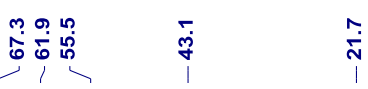
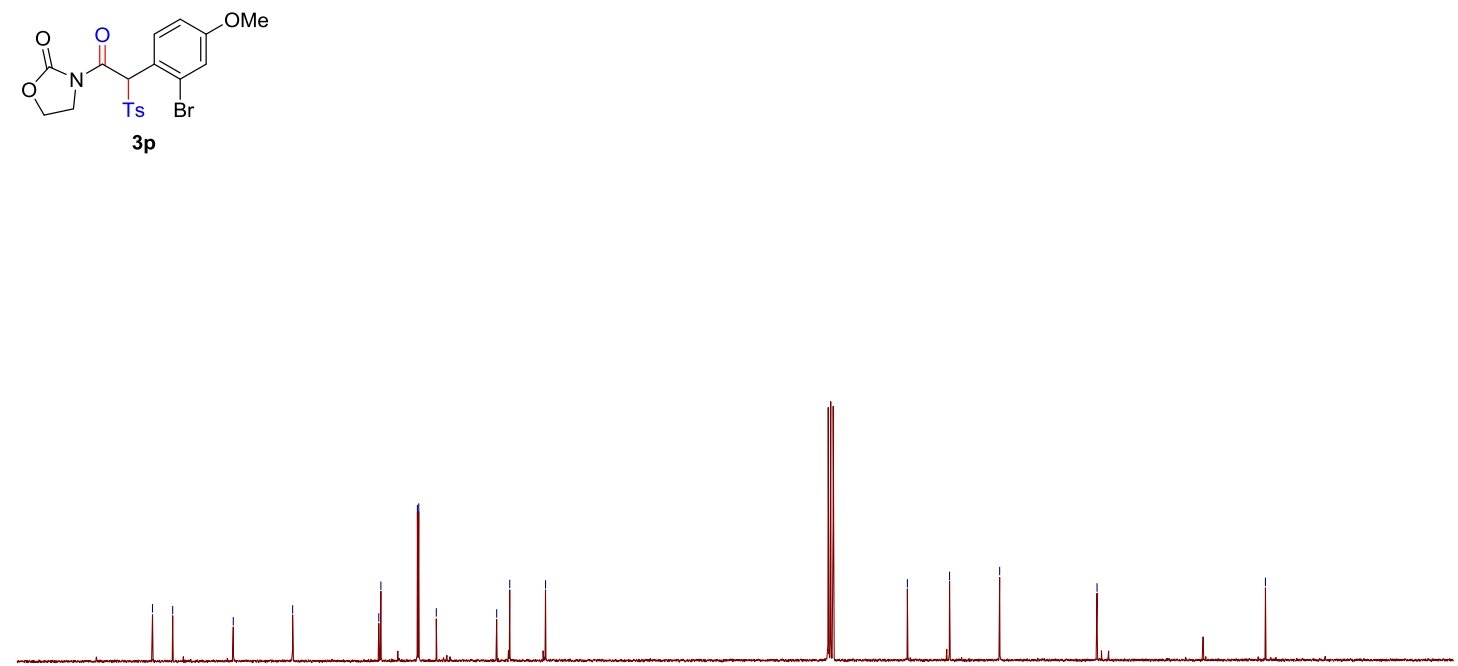

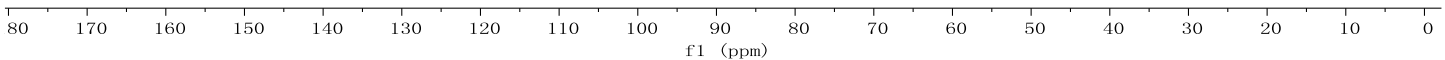




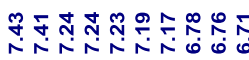

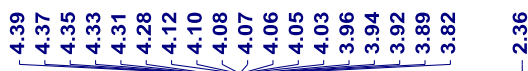

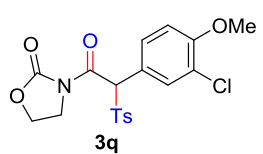

$3 q$

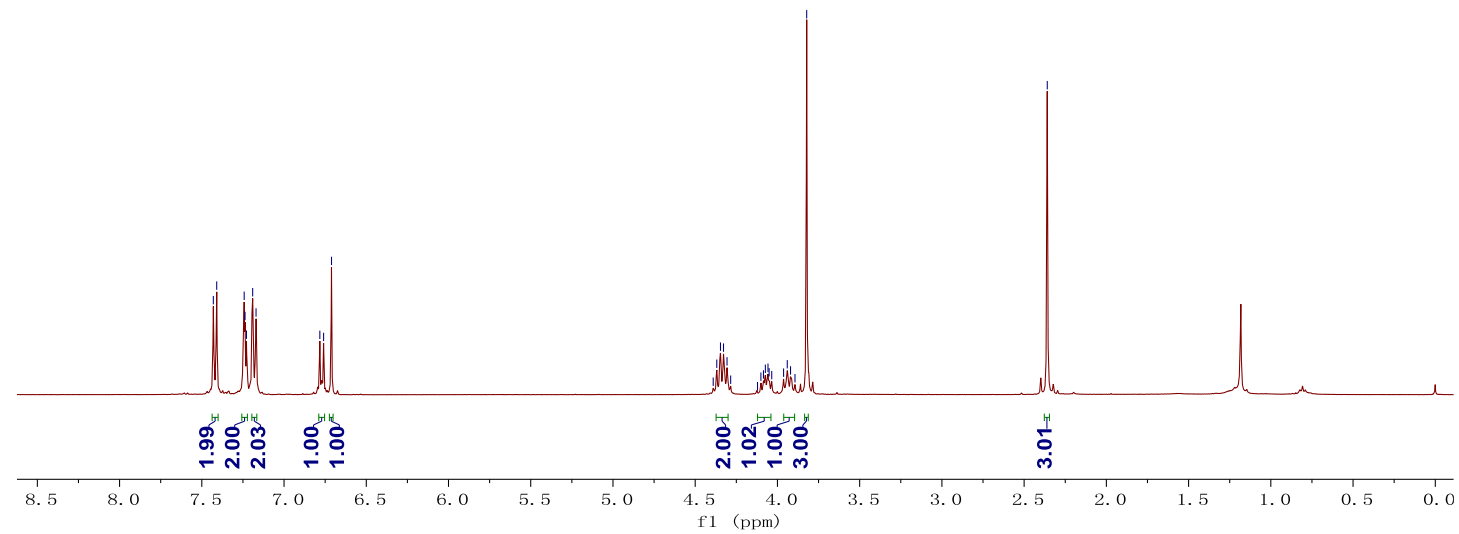

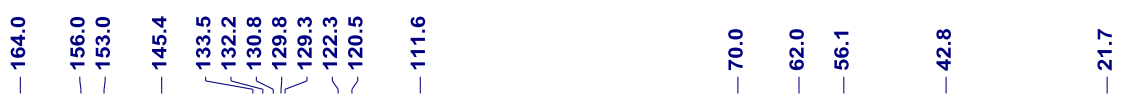
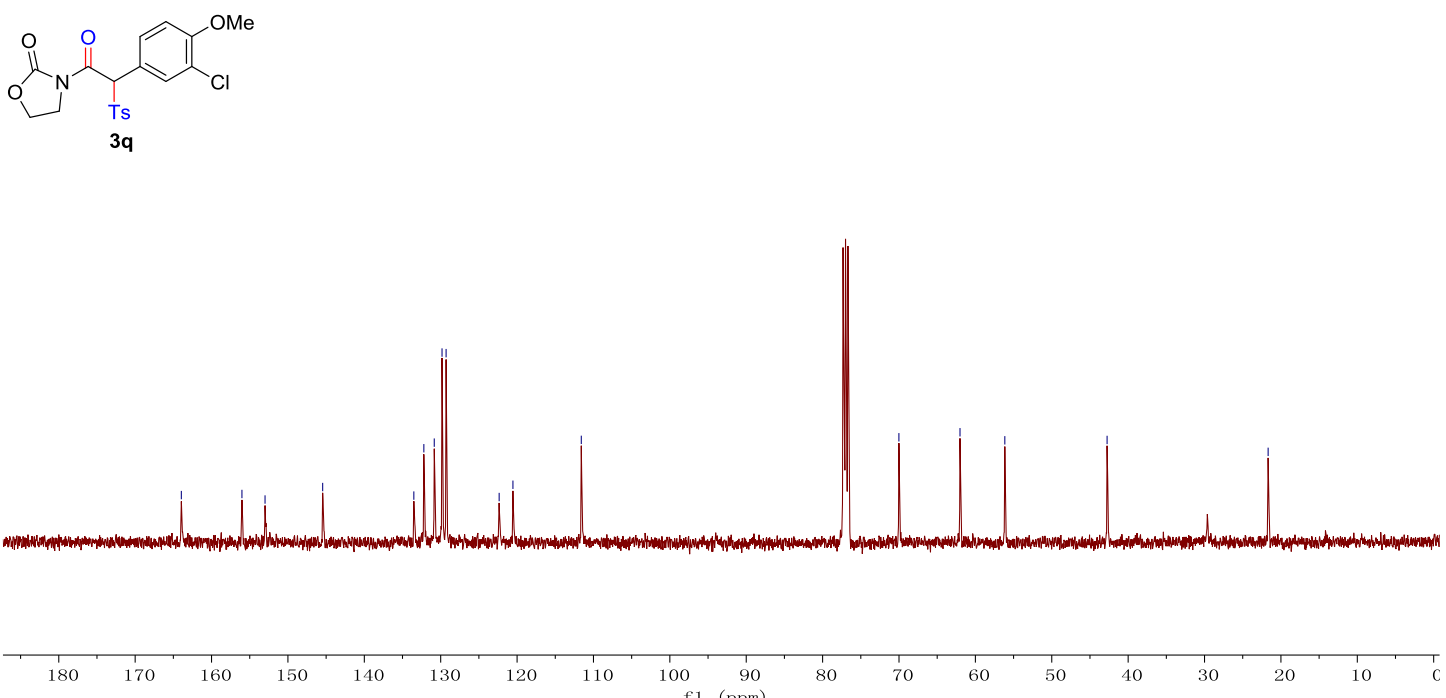

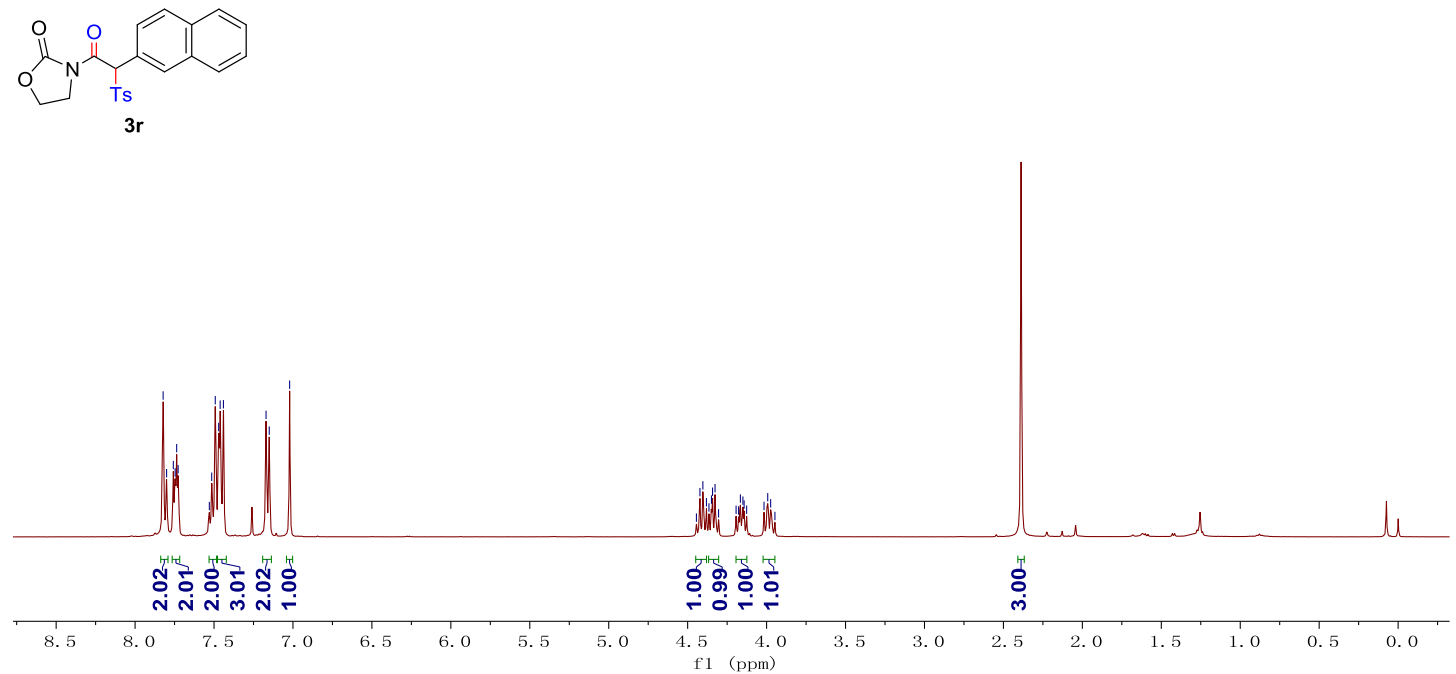

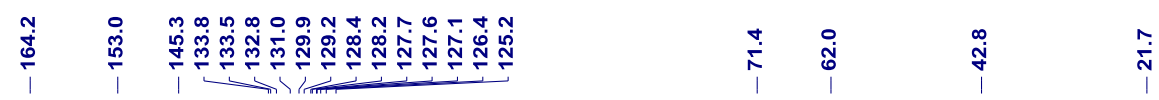
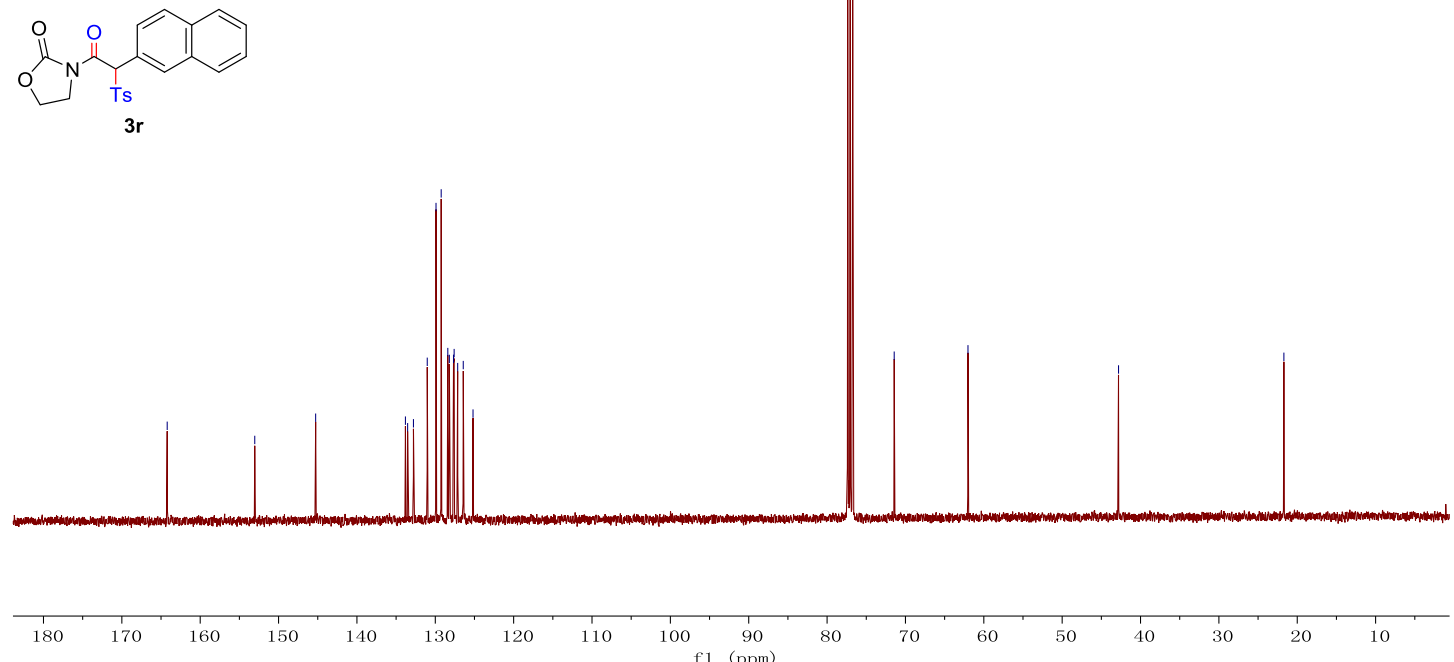


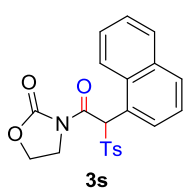

$3 \mathrm{~s}$

要

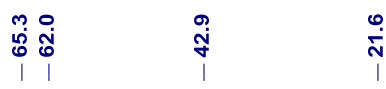

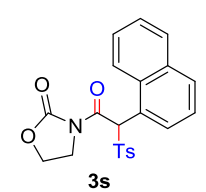

ynd hin

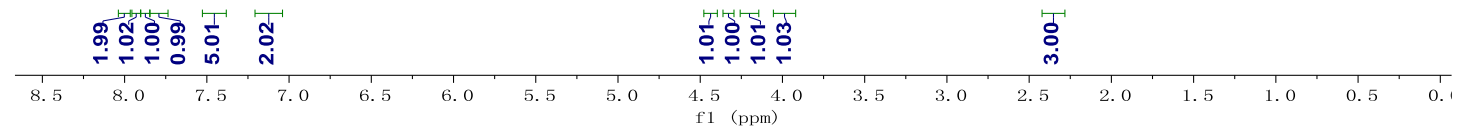

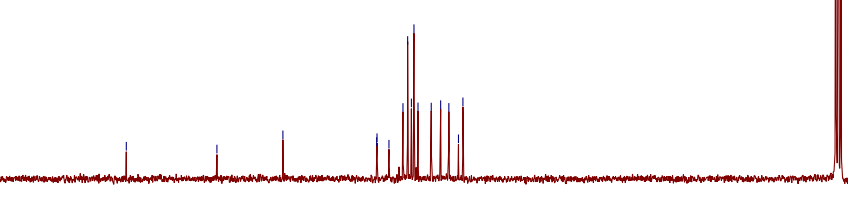

$80 \quad 170 \quad 160$ 

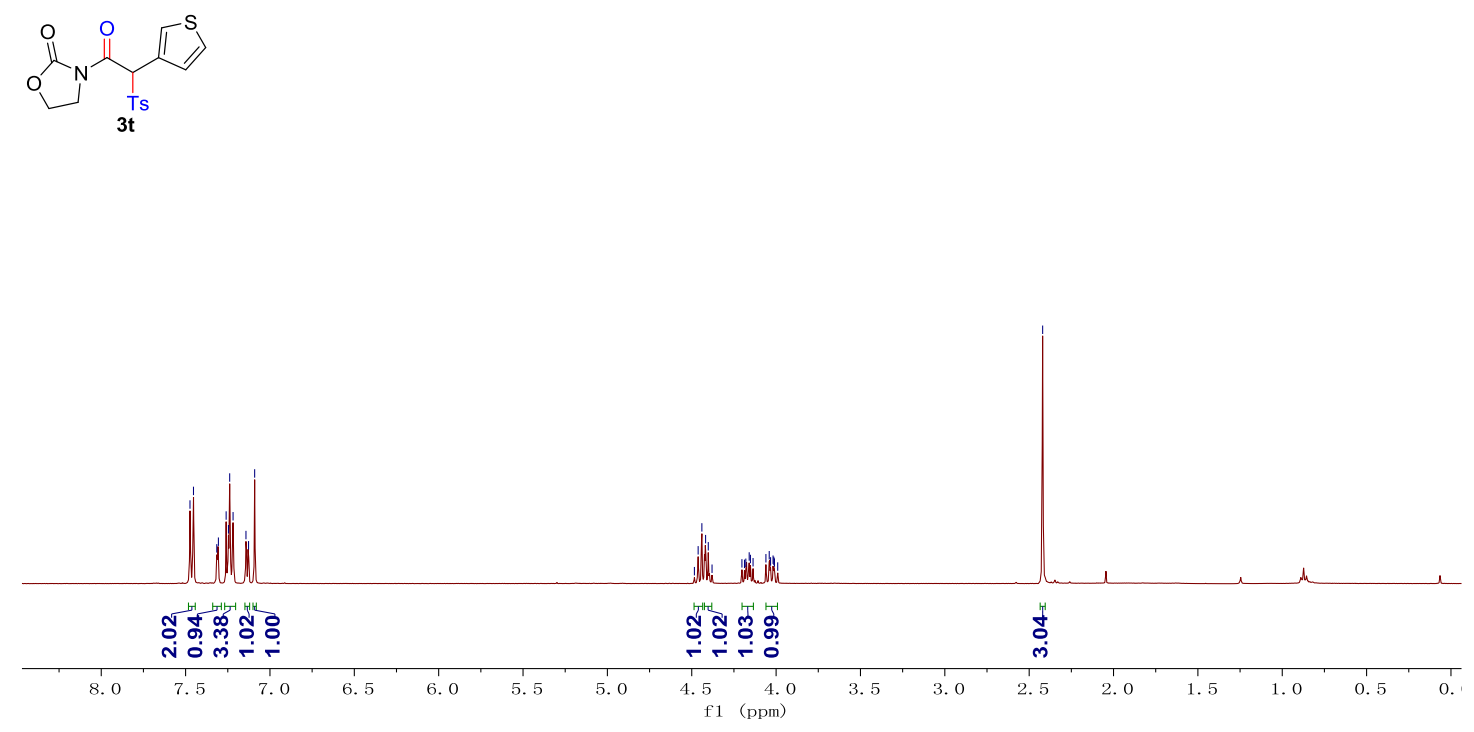
要

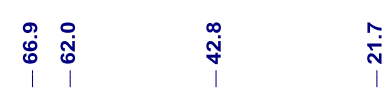
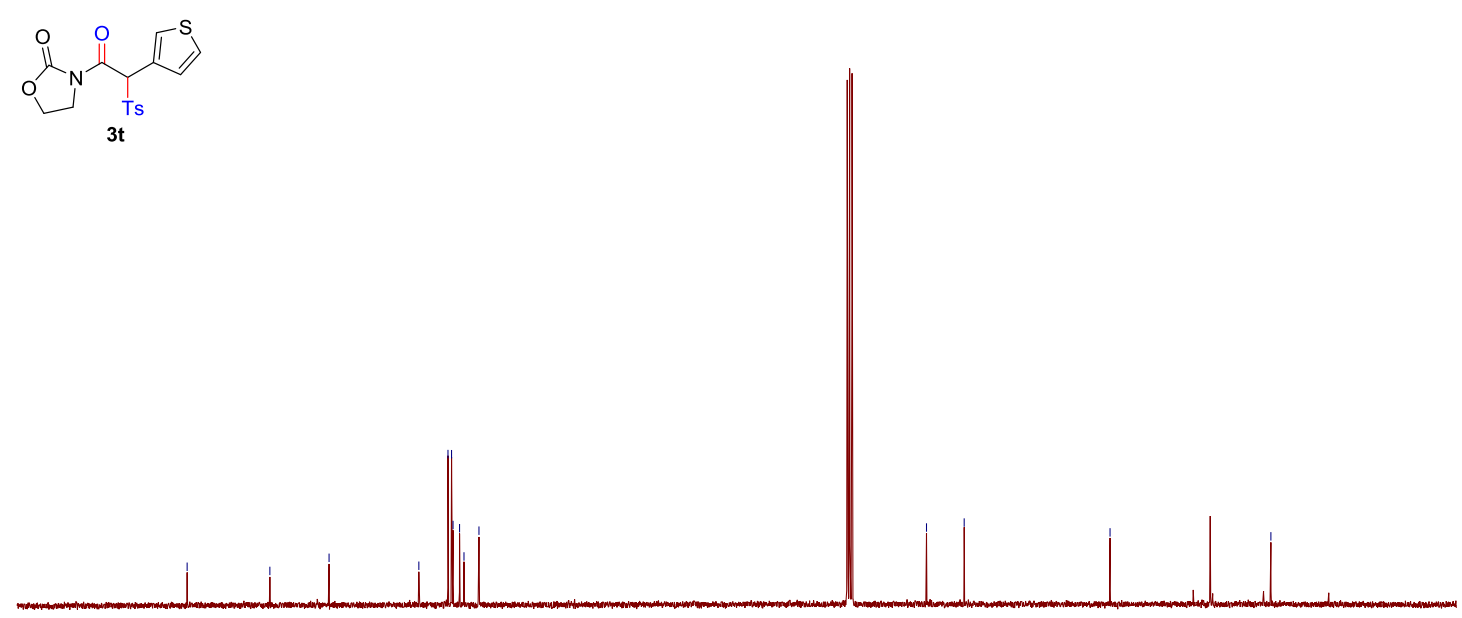

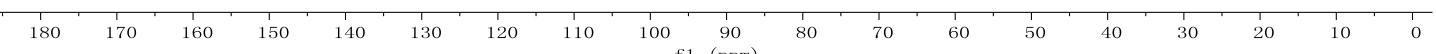




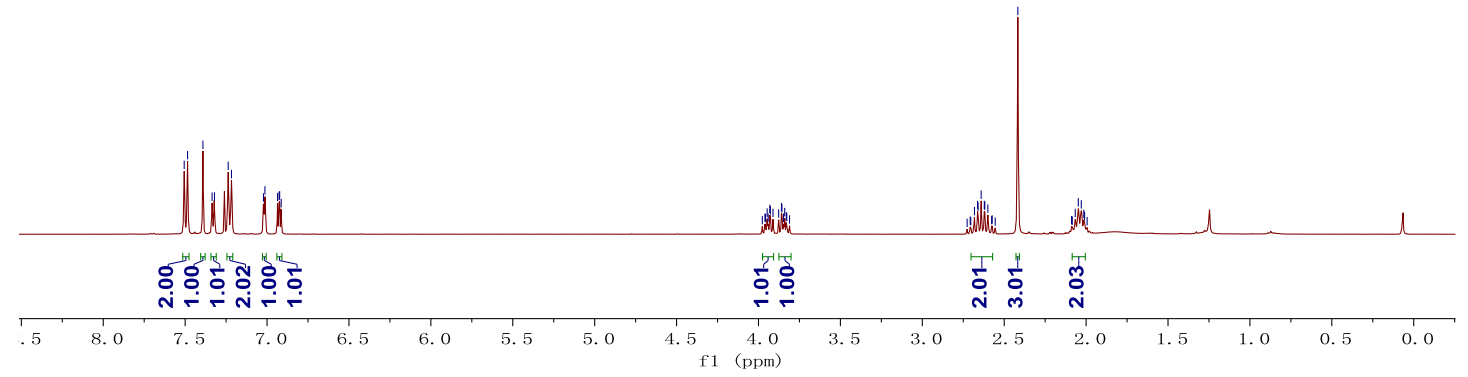

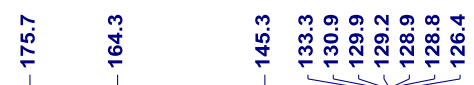

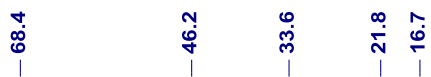

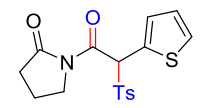

$3 \mathbf{u}$

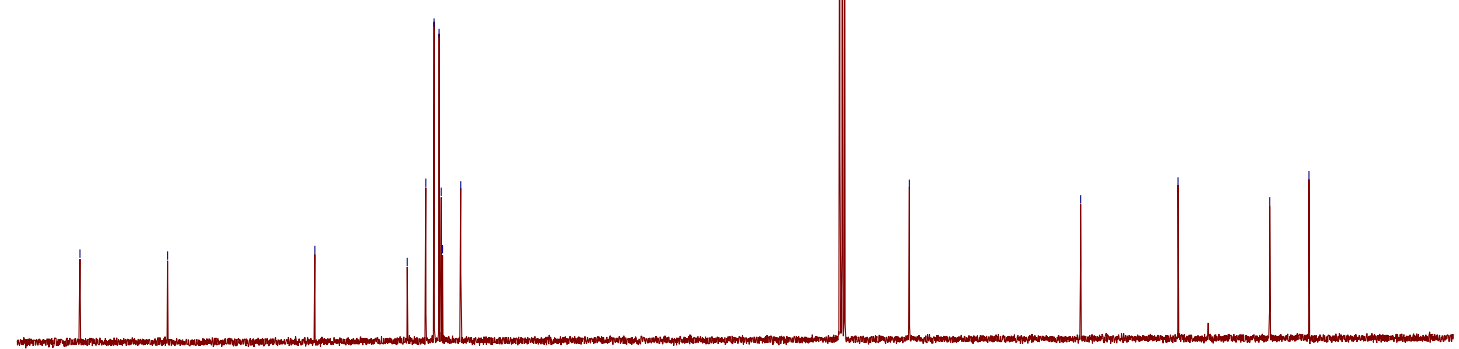

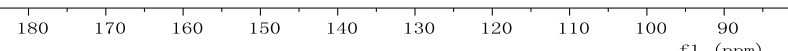



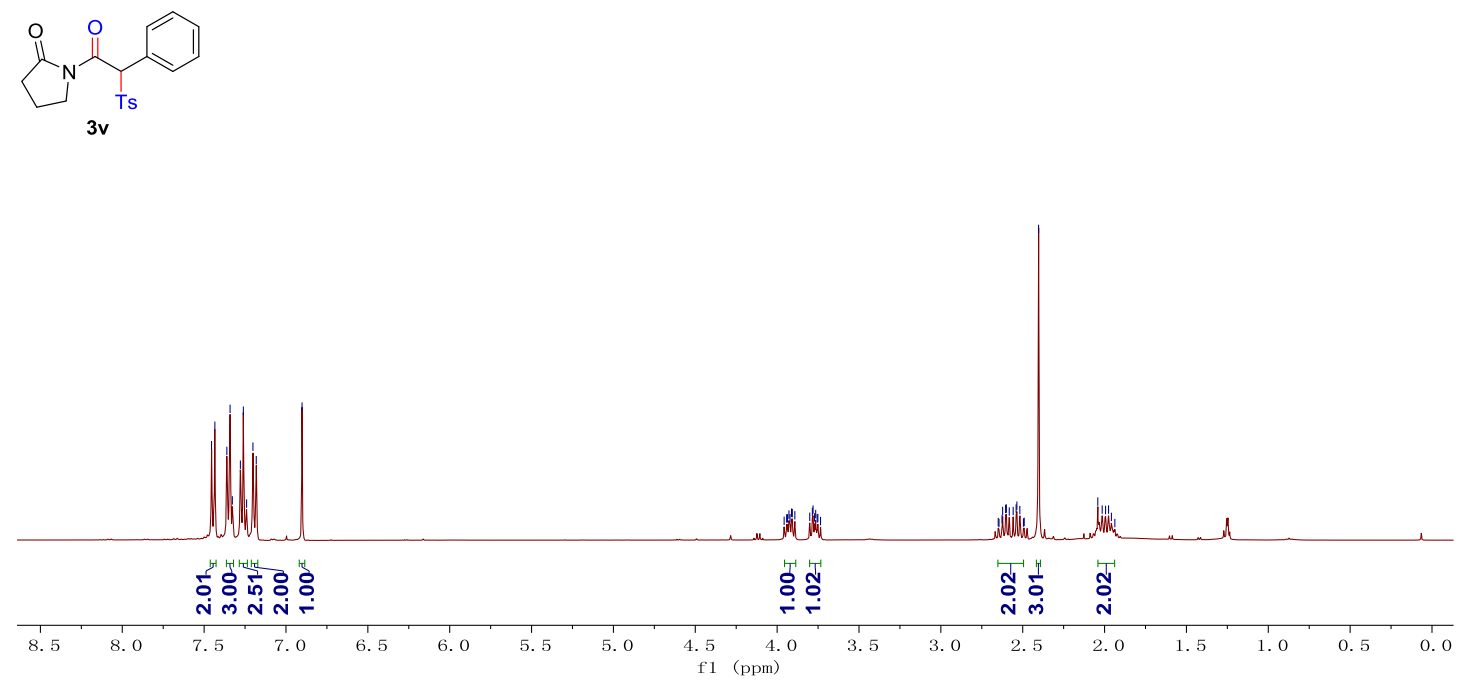

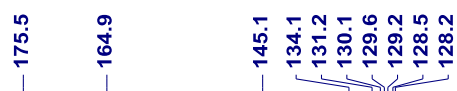

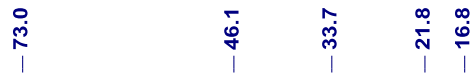

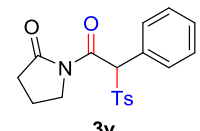

$3 v$

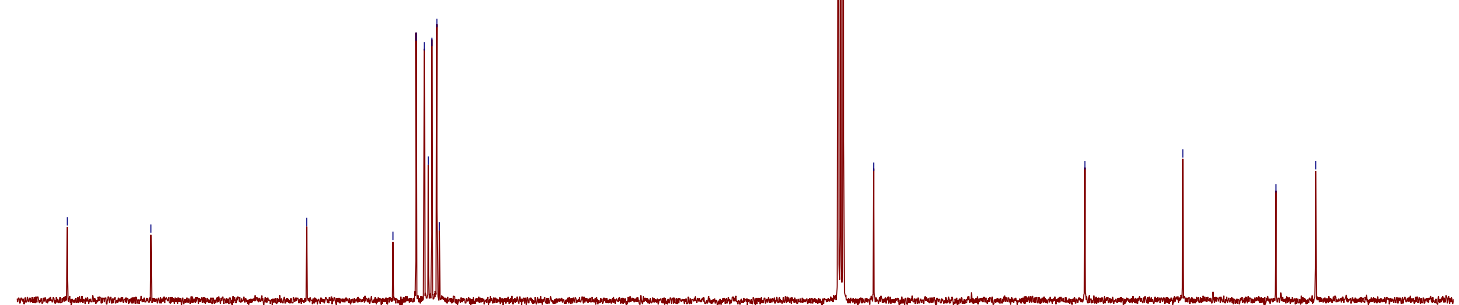

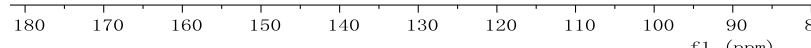




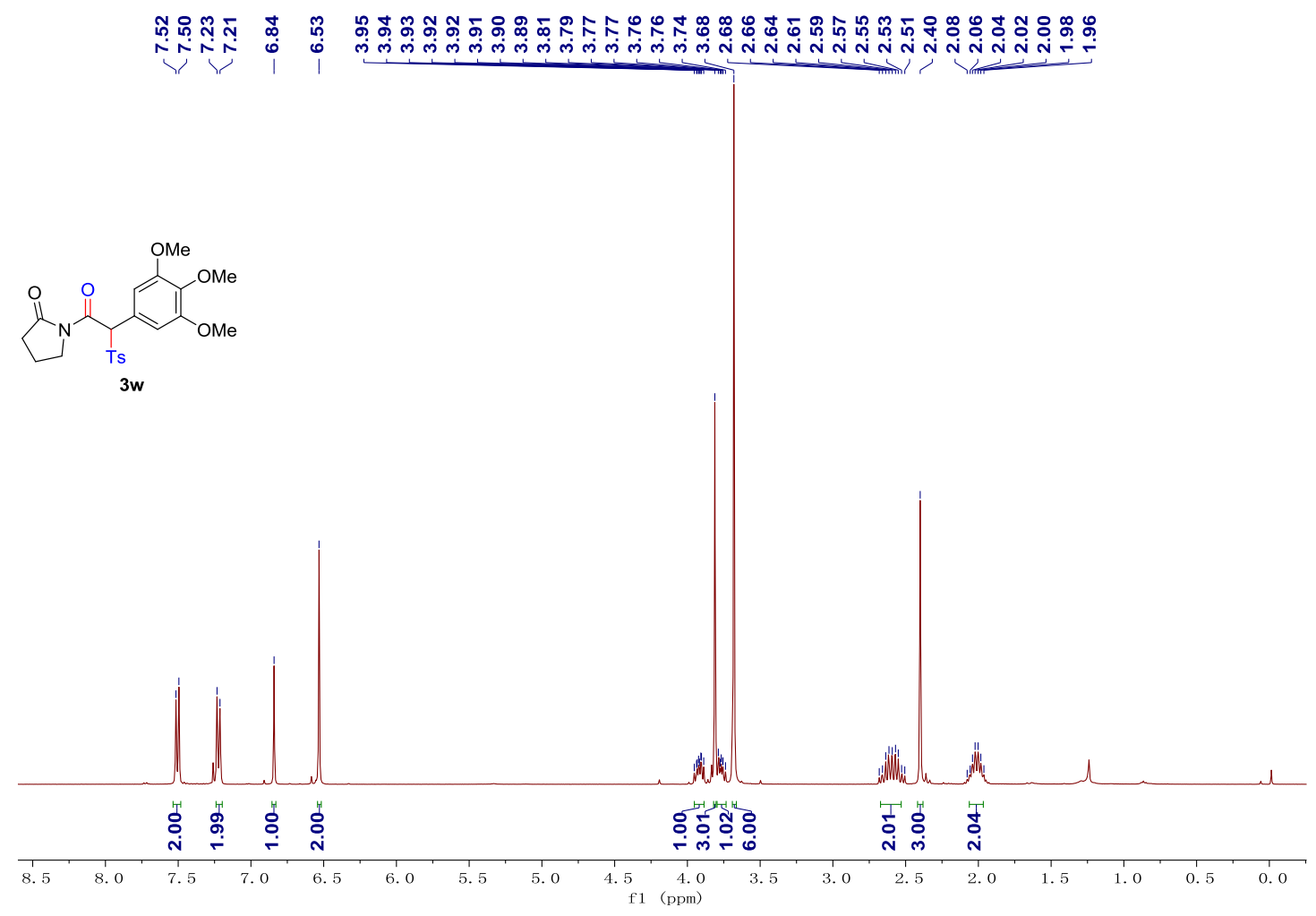

\section{常}
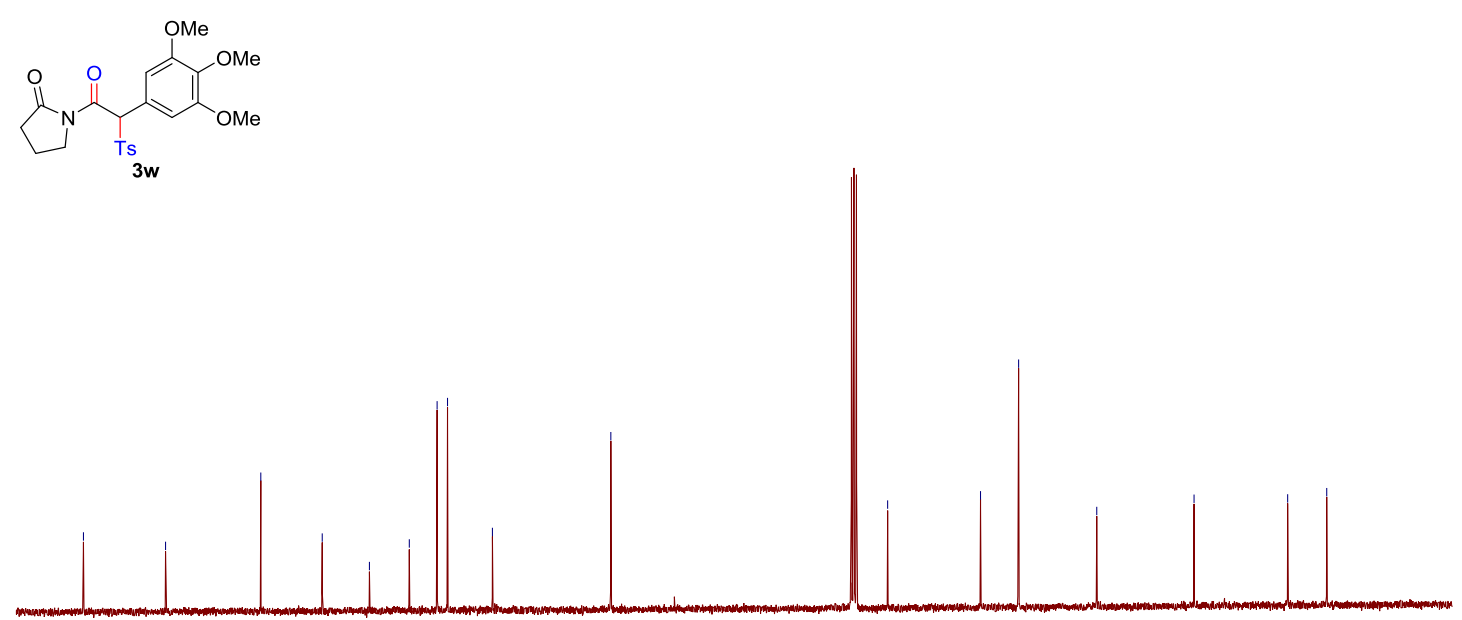

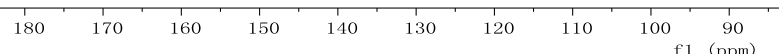



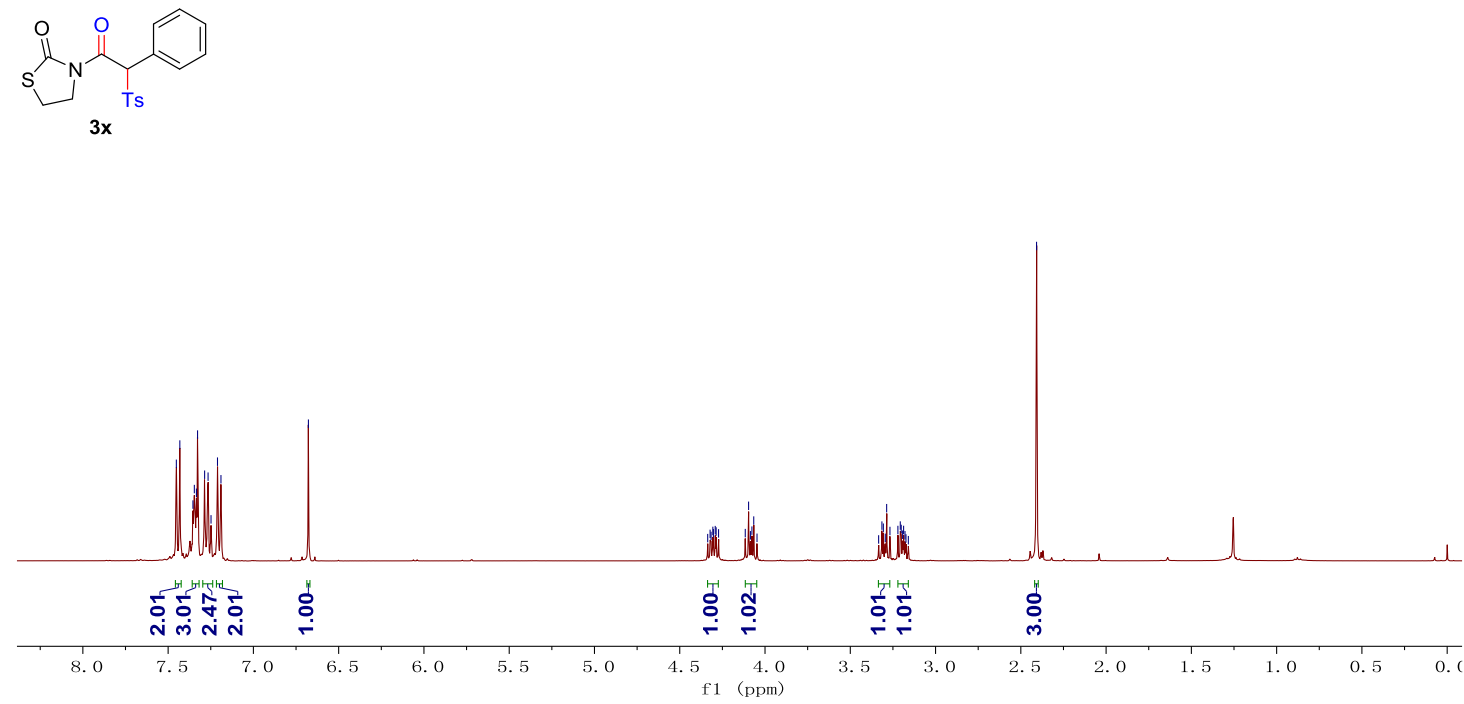

戛
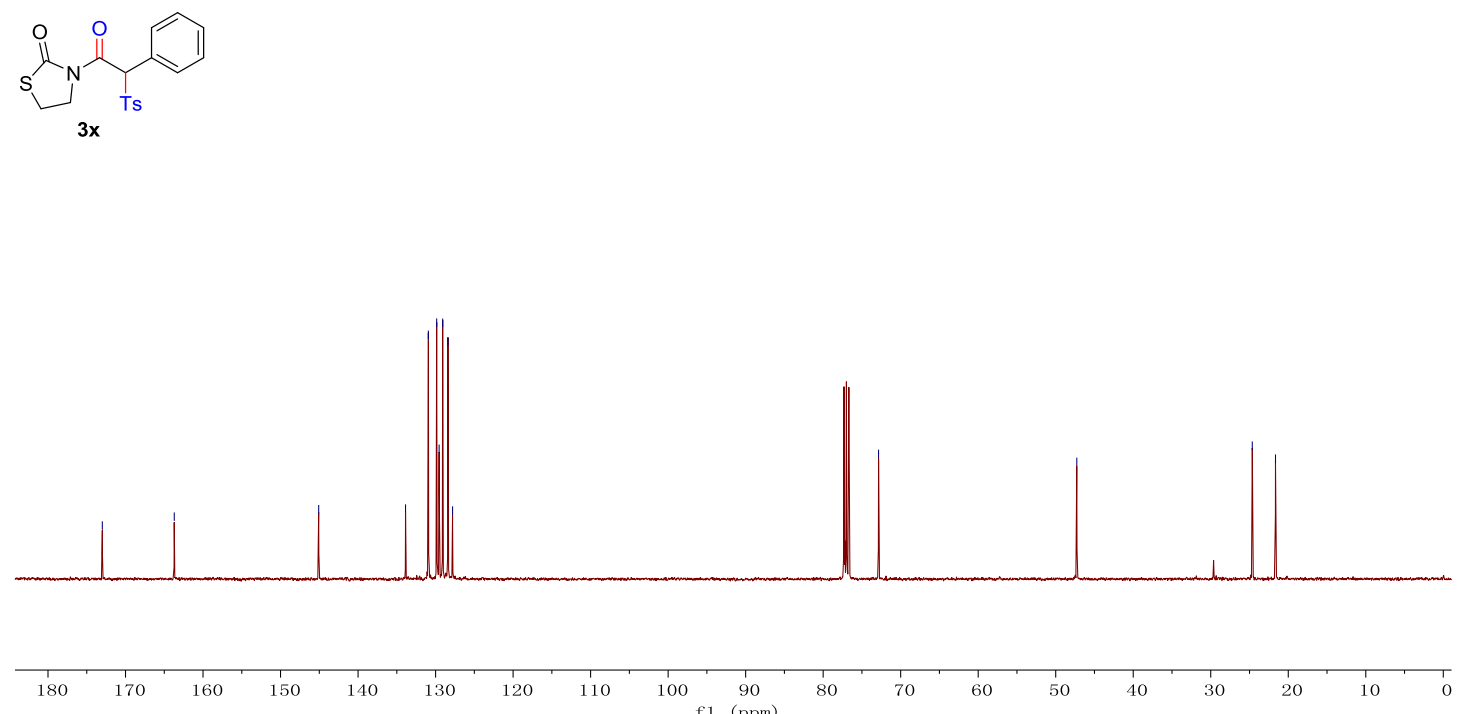


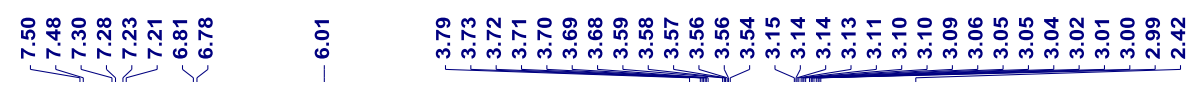

$\underbrace{O}_{\substack{3 y \\ 3 y}}$

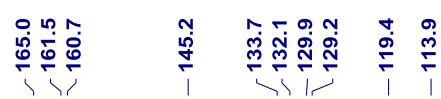

褊

$\sum_{\substack{3 y \\ \text { Ts }}}^{O}$

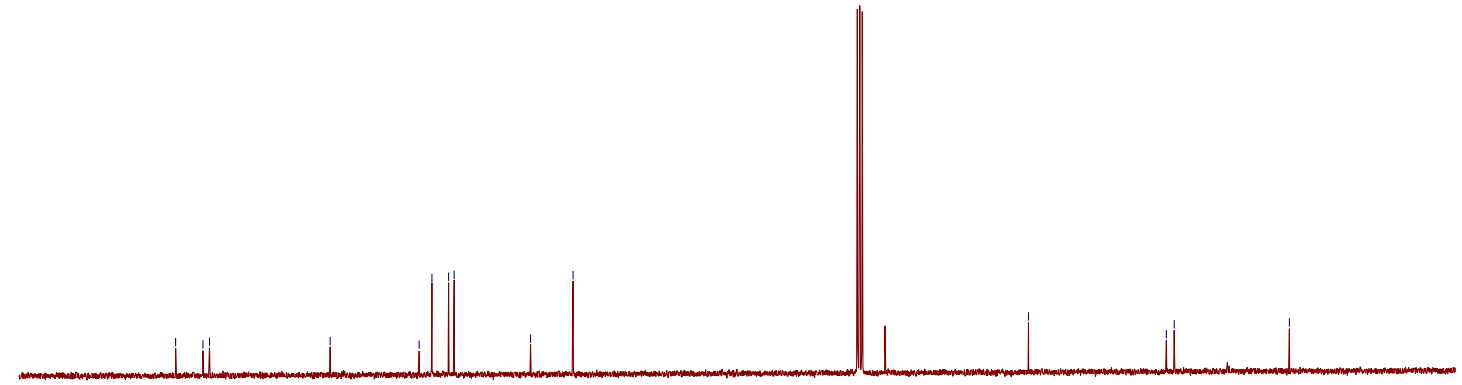

$180 \quad 170 \quad 160$

$100 \quad 90$ 

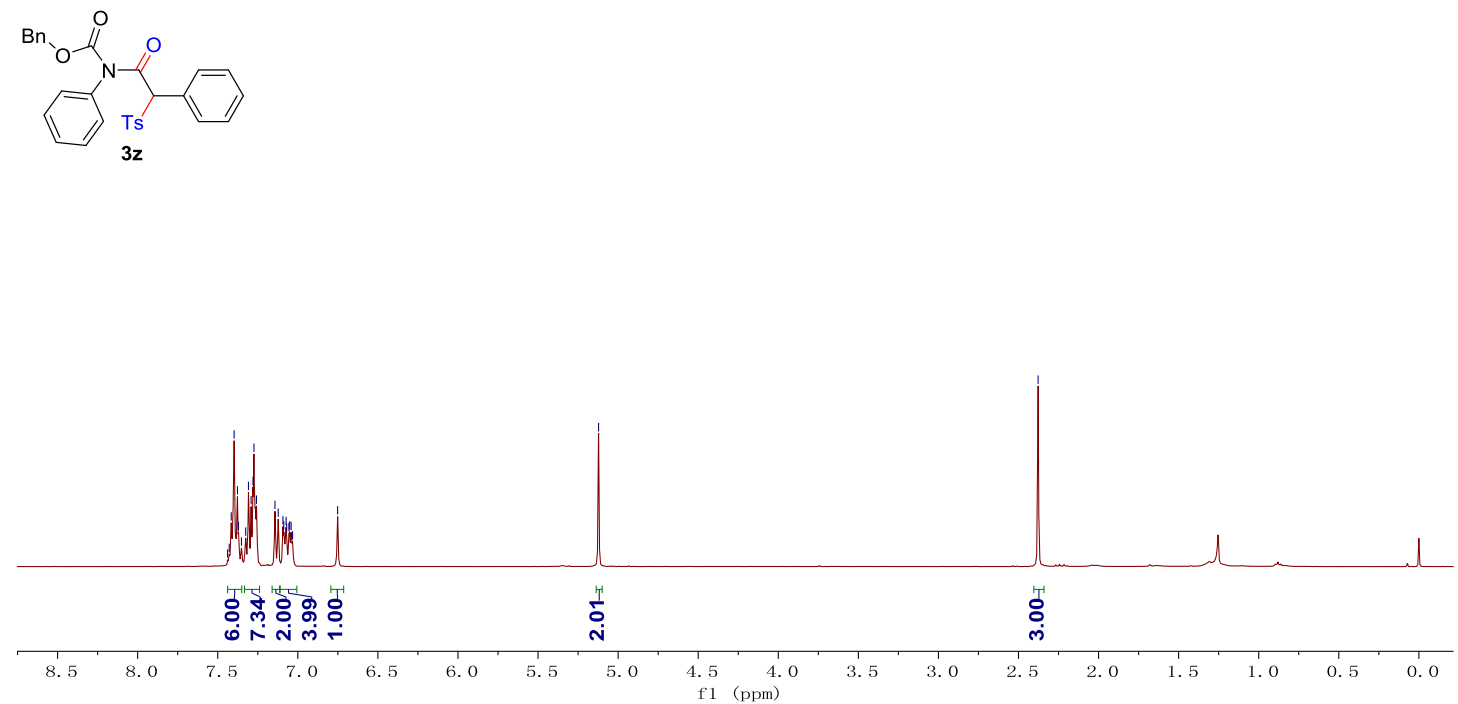

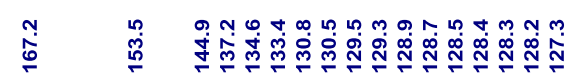

官

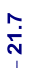
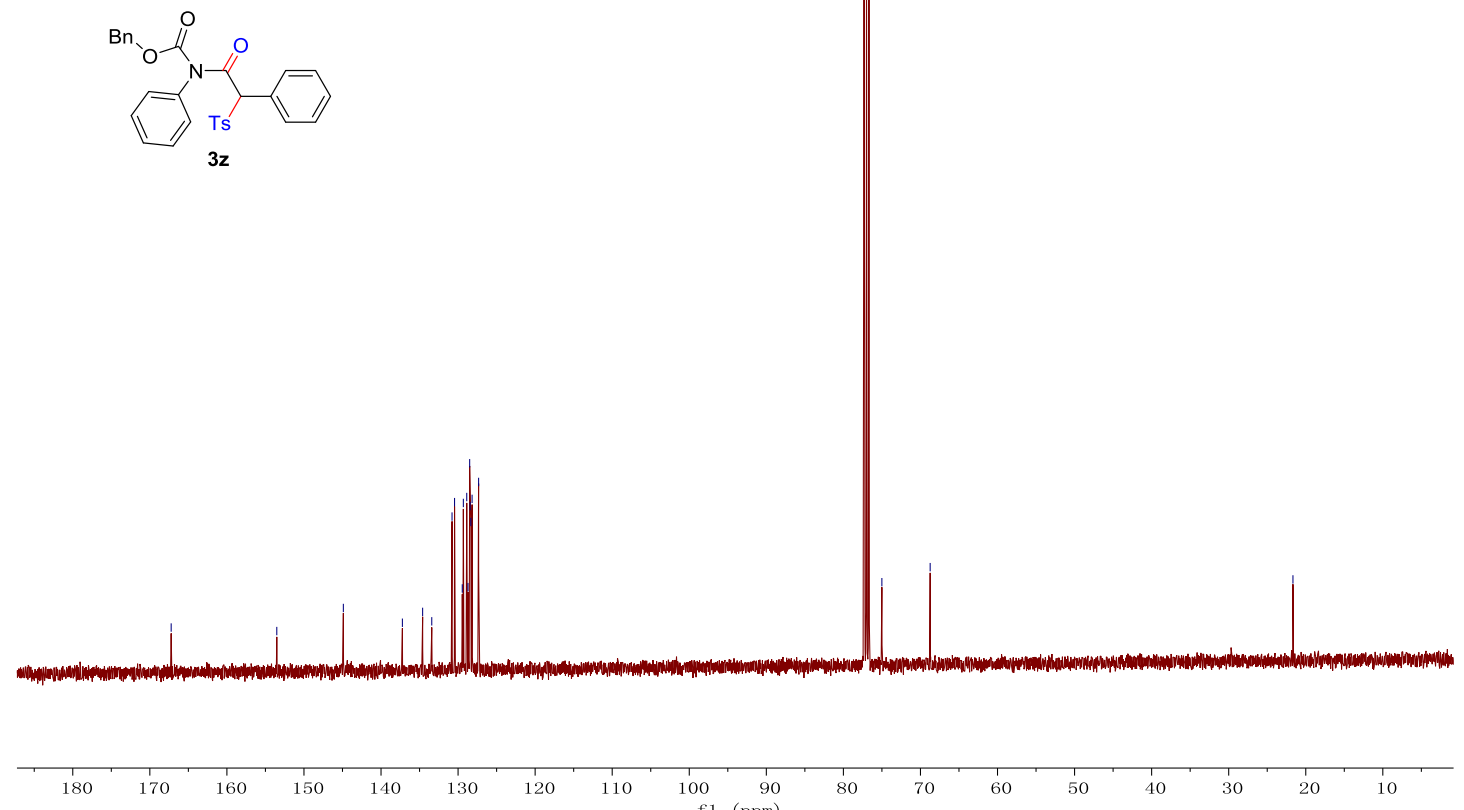

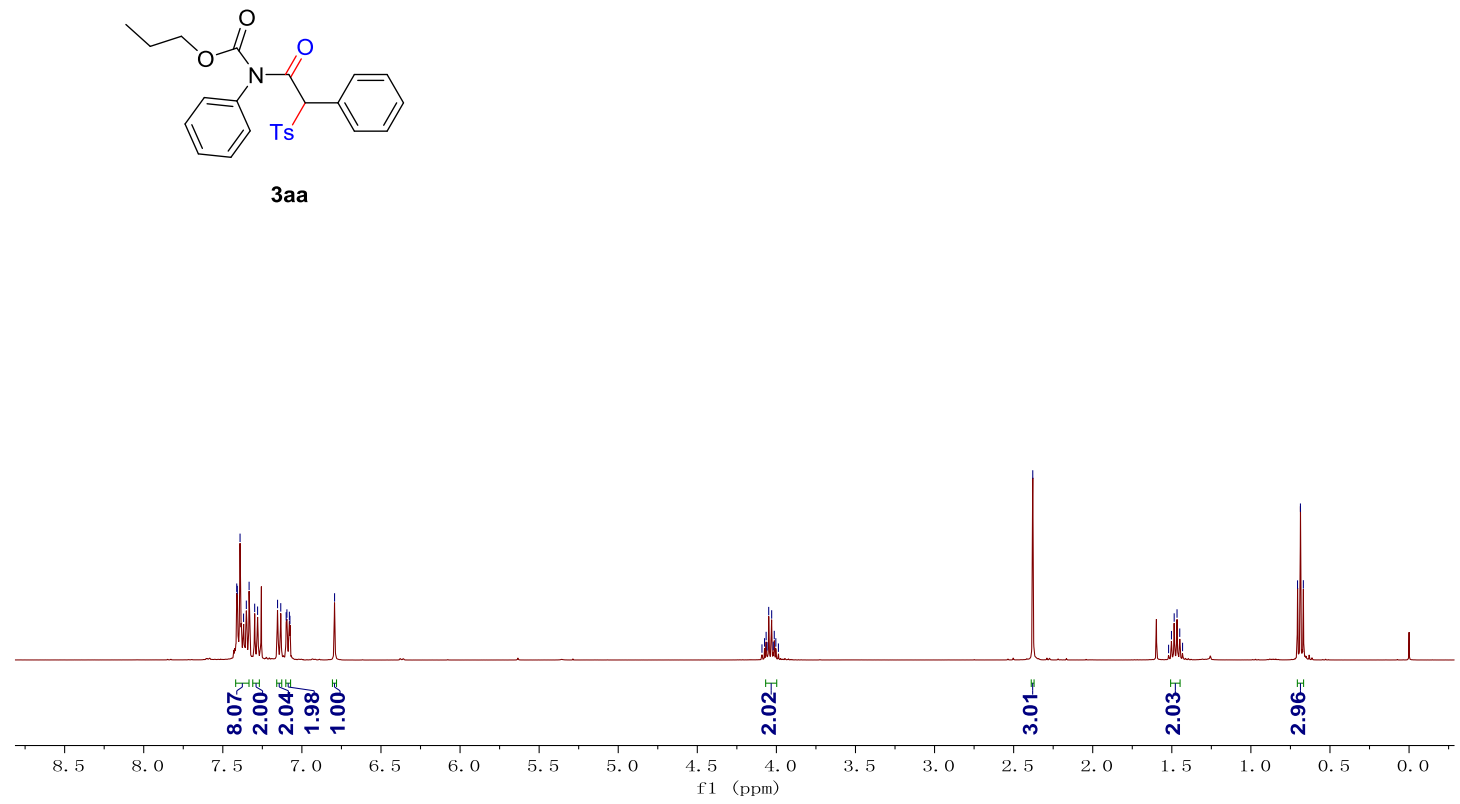

$\sqrt{1}$

i.

$\stackrel{\circ}{i} \underset{i}{i}$
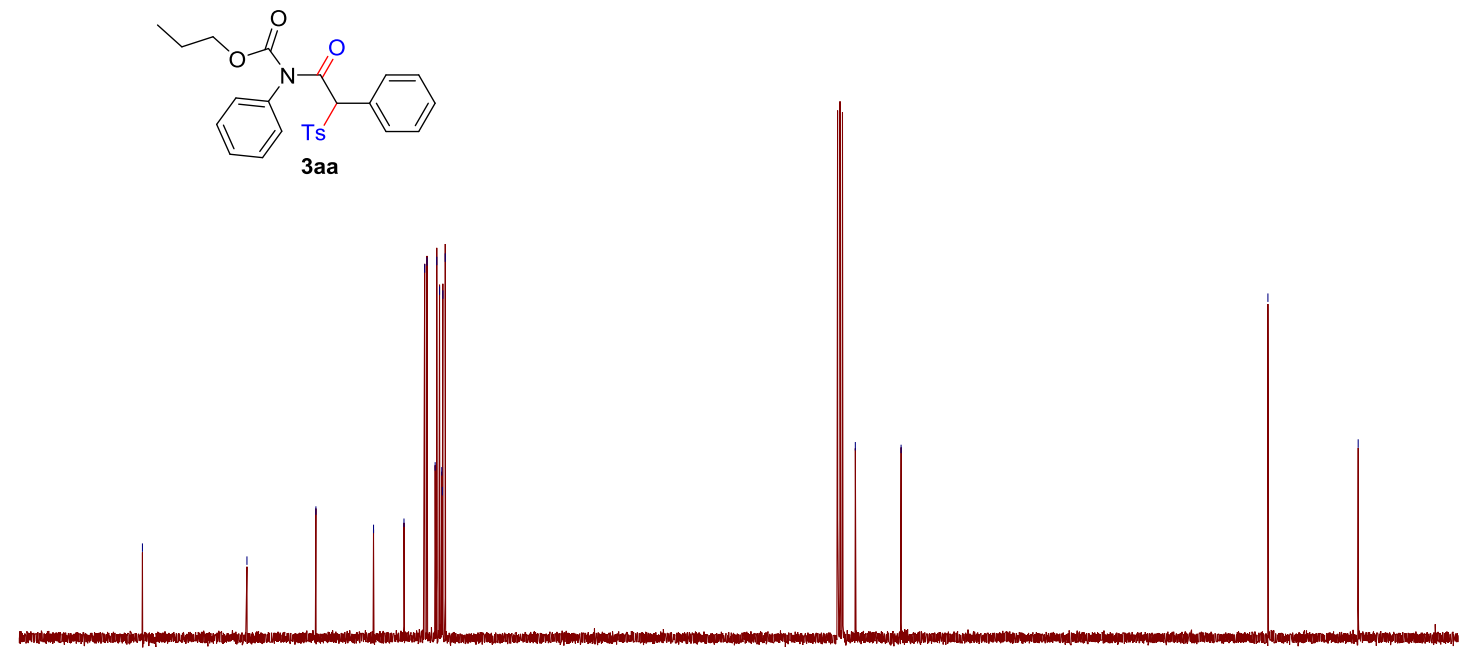

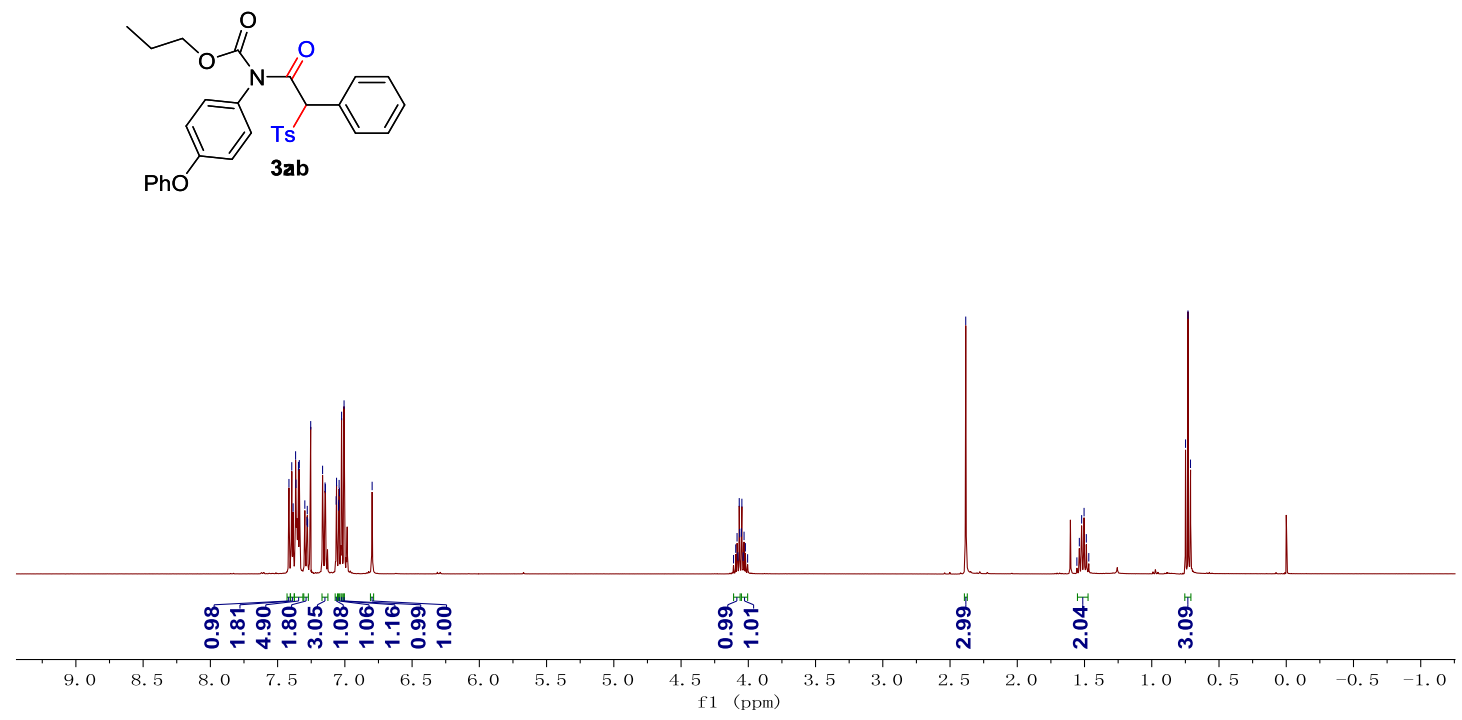

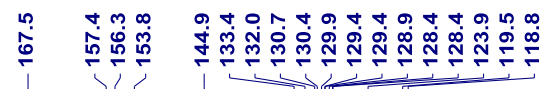

웅용

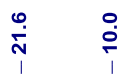

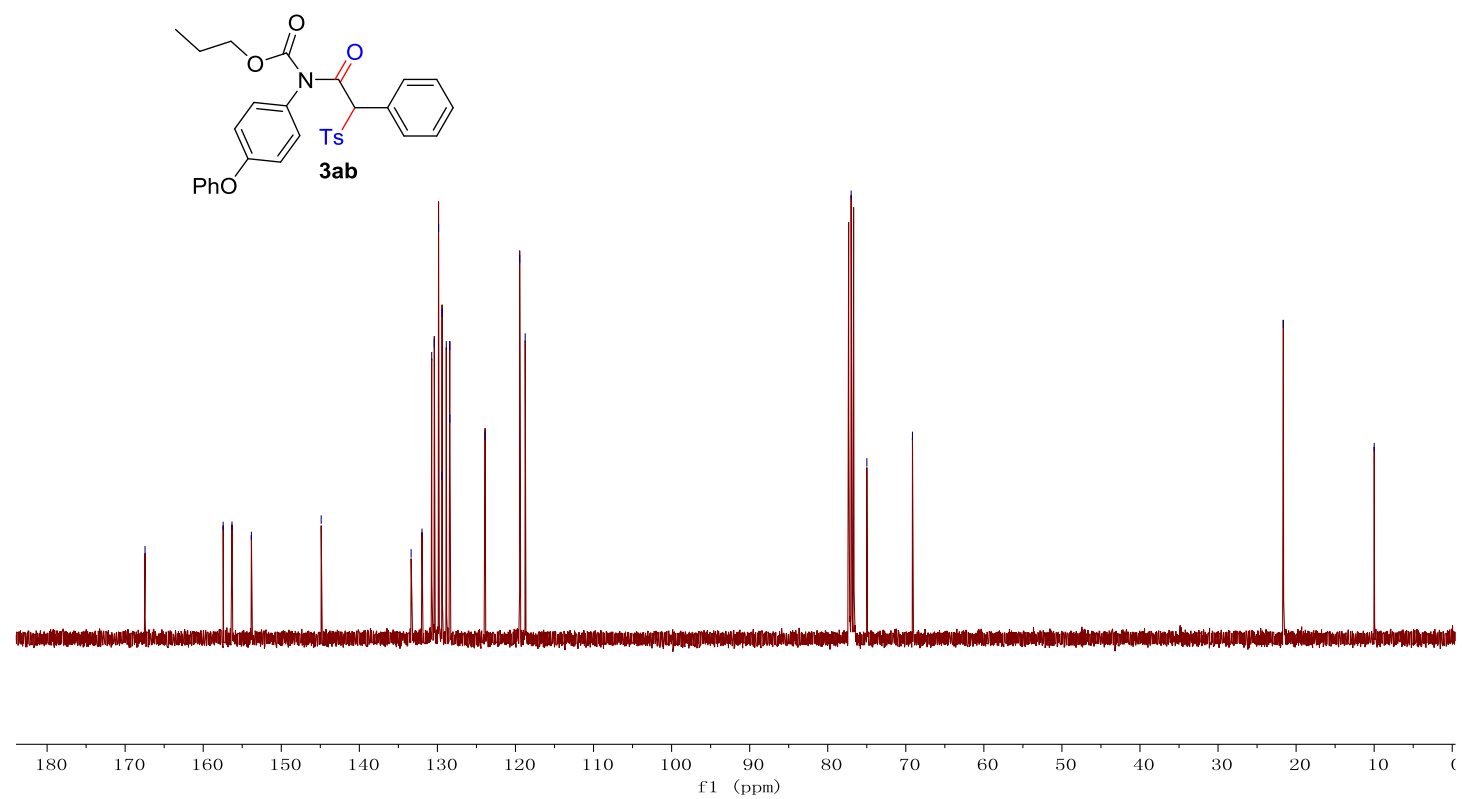




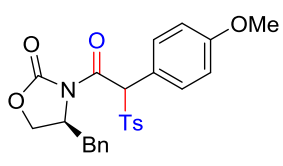

3ac, $\mathrm{dr}=2: 1$

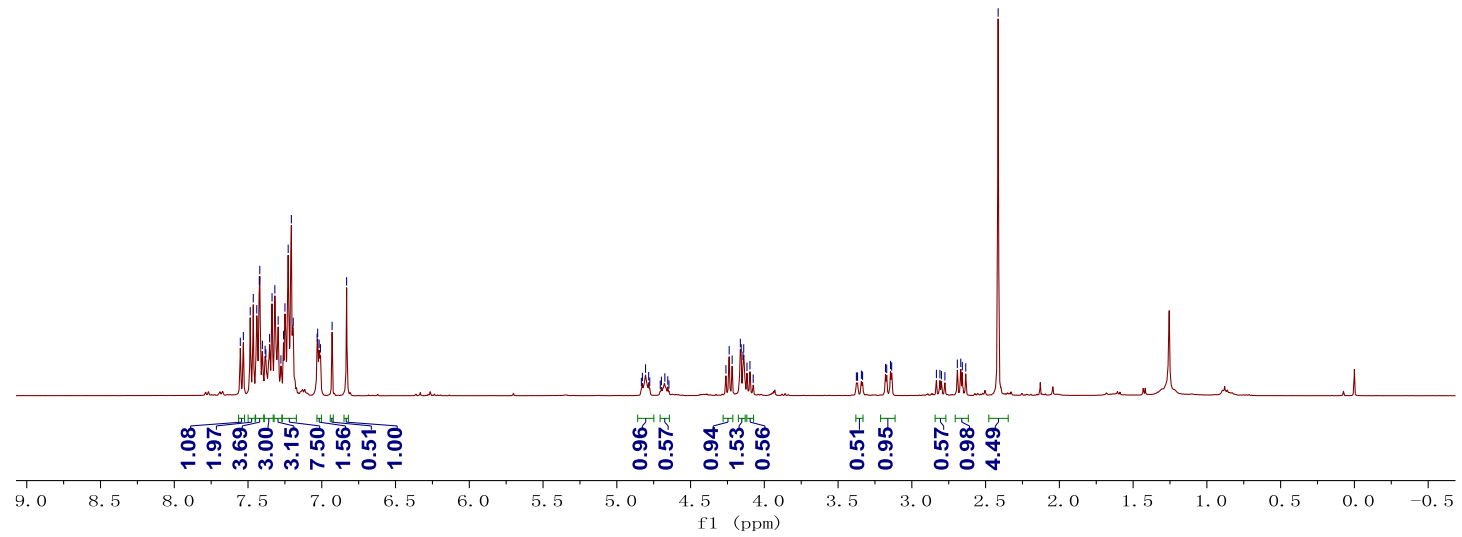

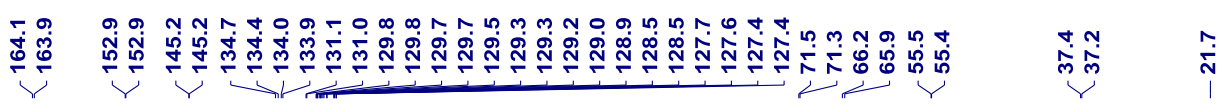

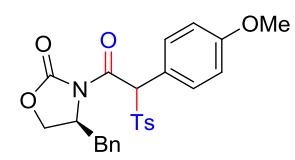

3ac, $d r=2: 1$

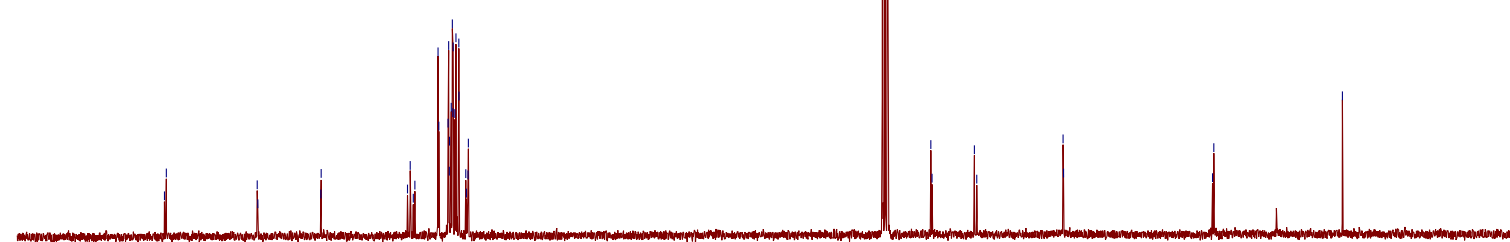

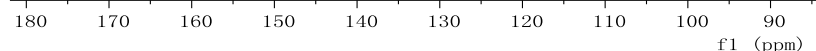




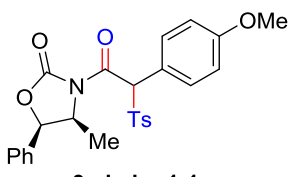

3ad, $\mathrm{dr}=1: 1$
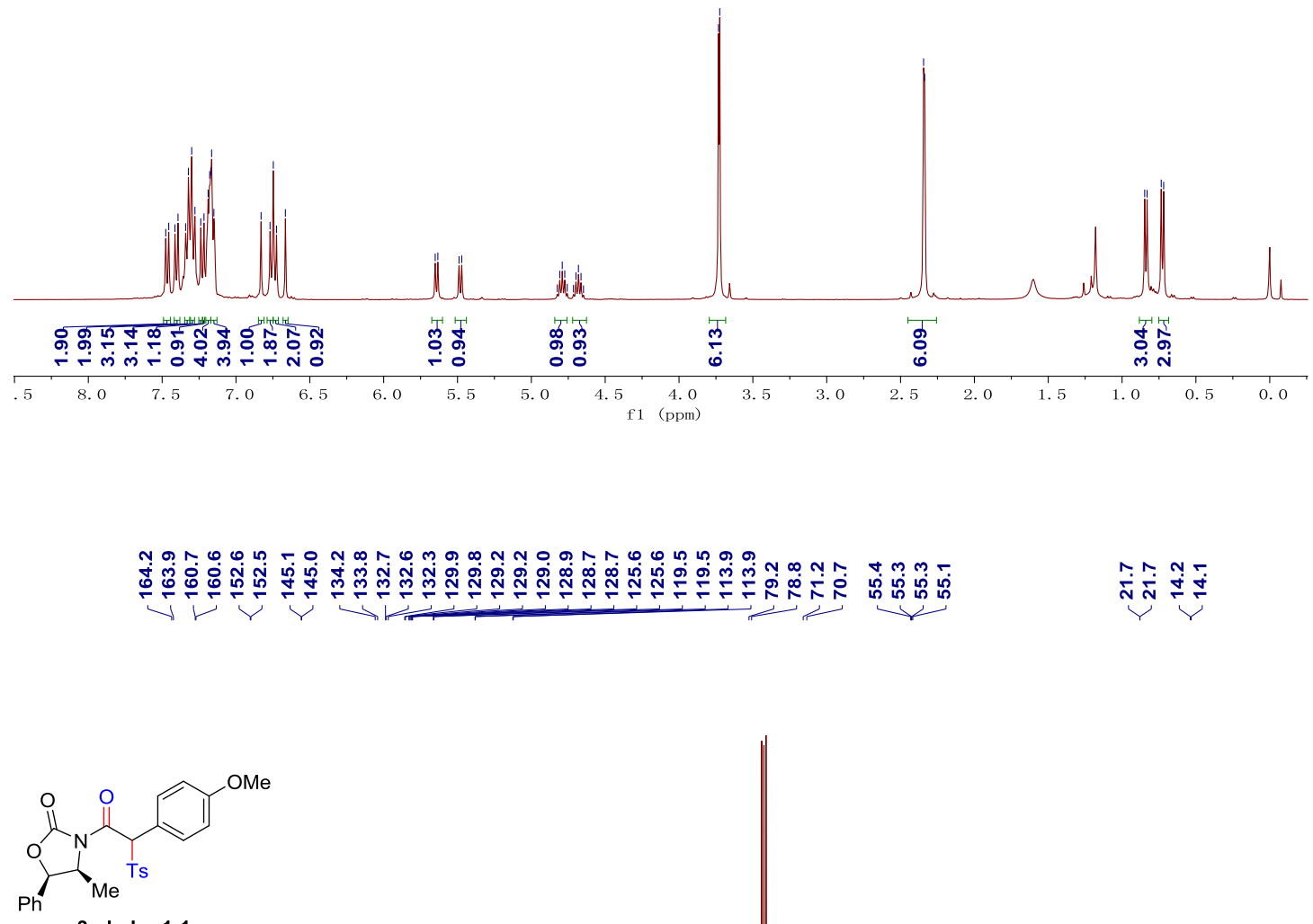

3ad, dr= 1:1

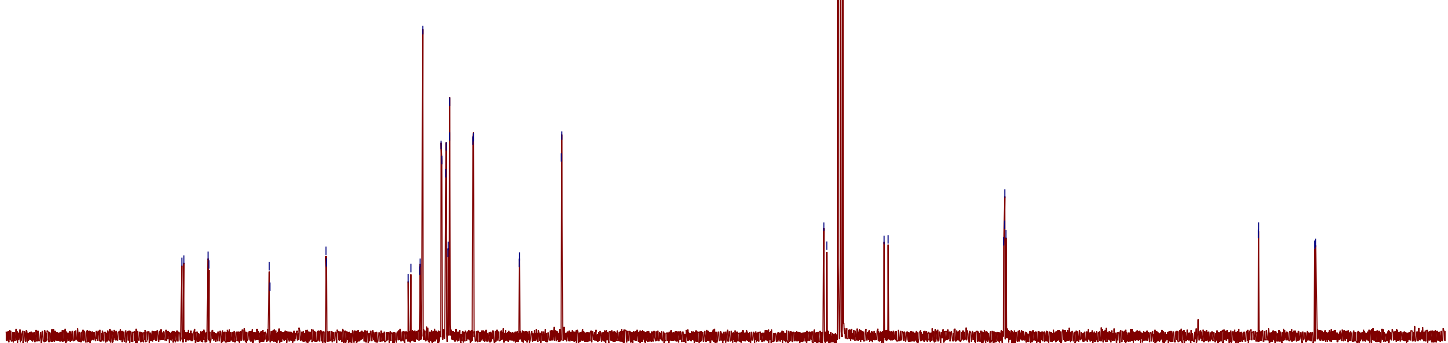

$\begin{array}{llllllllll}180 & 170 & 160 & 150 & 140 & 130 & 120 & 110 & 100 & 90\end{array}$

$80 \quad 70 \quad 60$

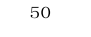

$2010 \quad 0$ 


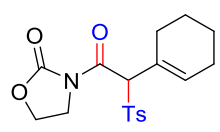

3ae

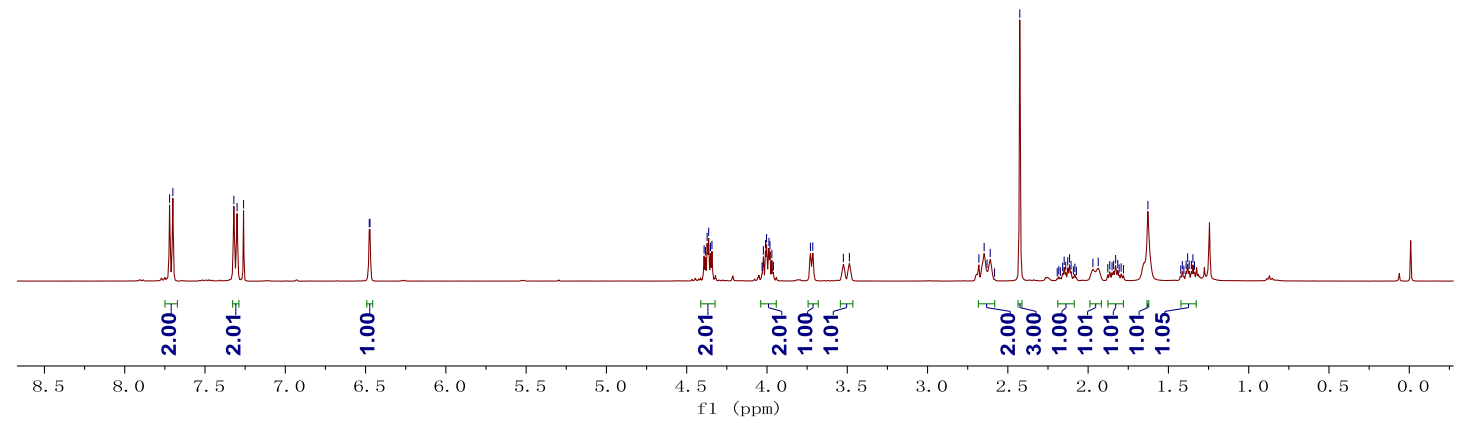

㶾

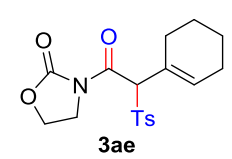

3ae

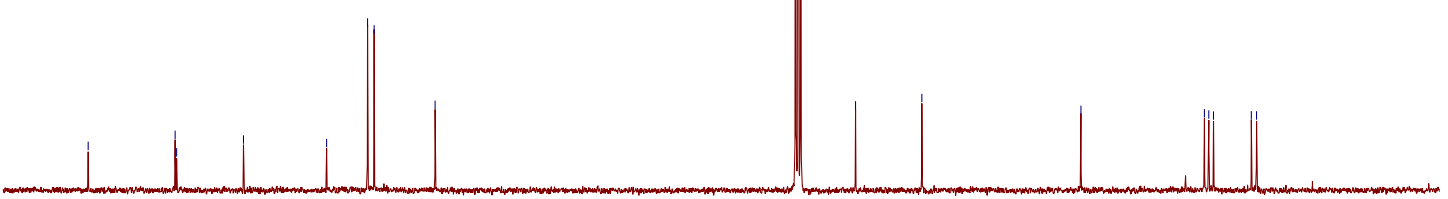

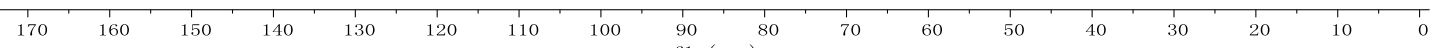



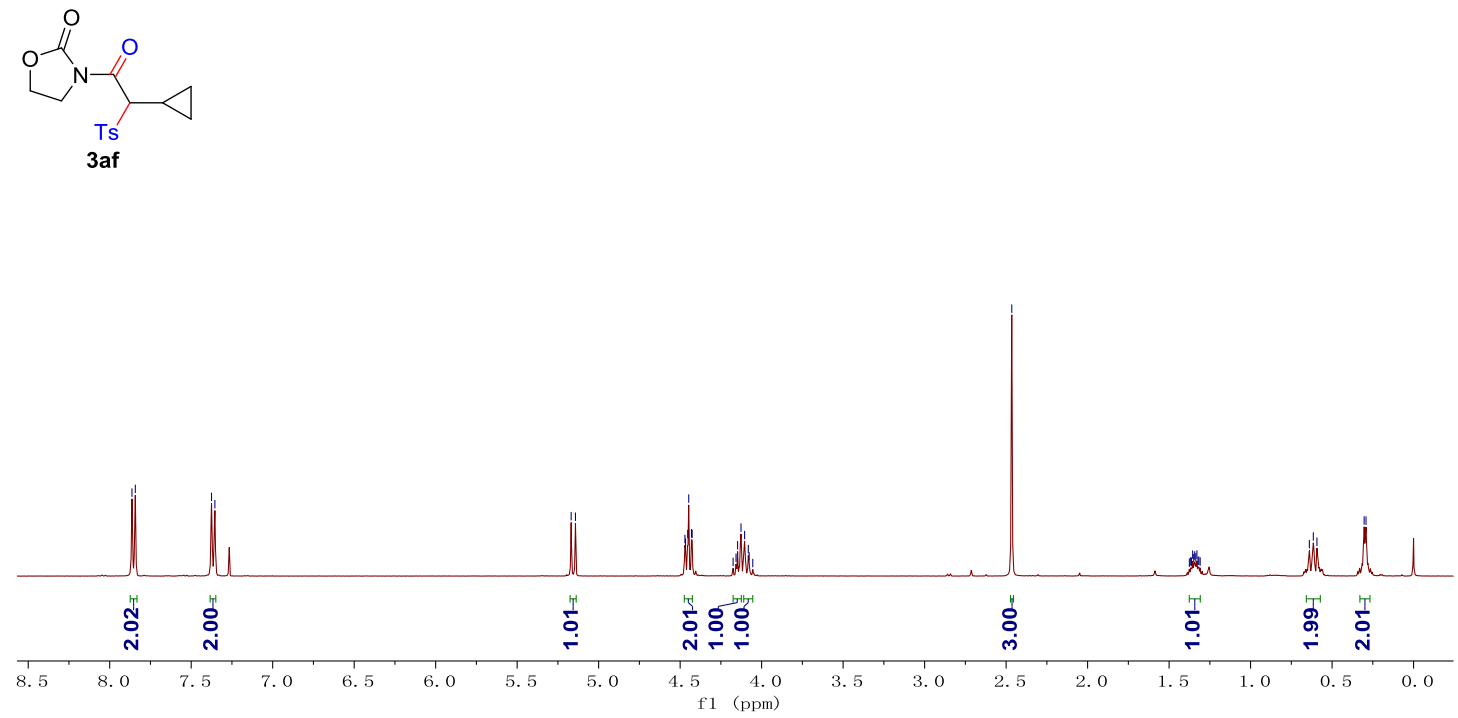

$\underbrace{\mathrm{N}-4}_{\substack{\text { Ts } \\ \text { 3af }}}$

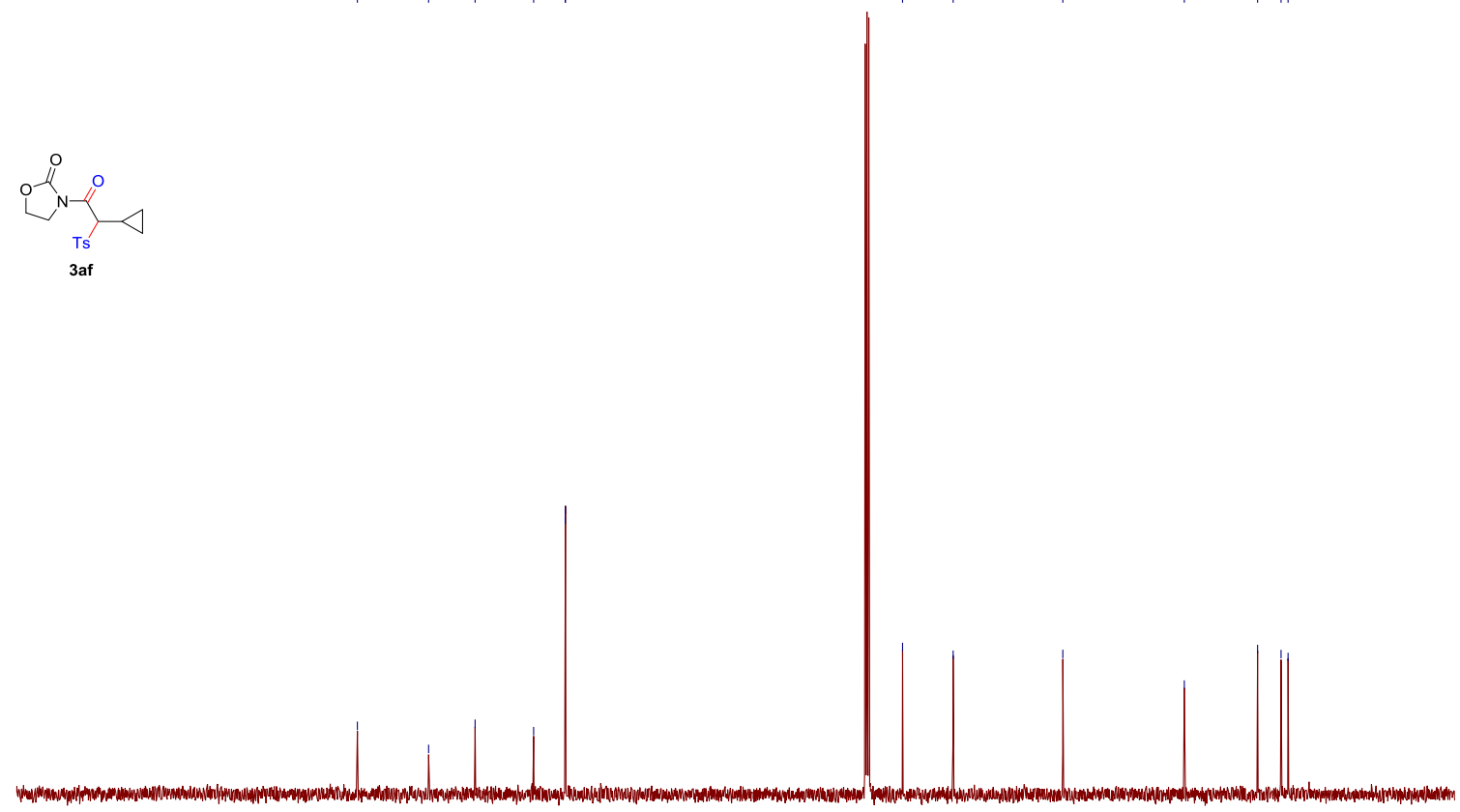

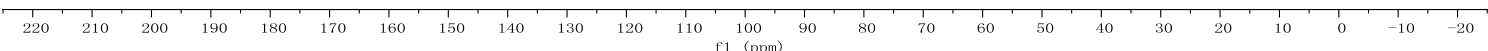


$\underbrace{O}_{3 \mathbf{3 a g}}$
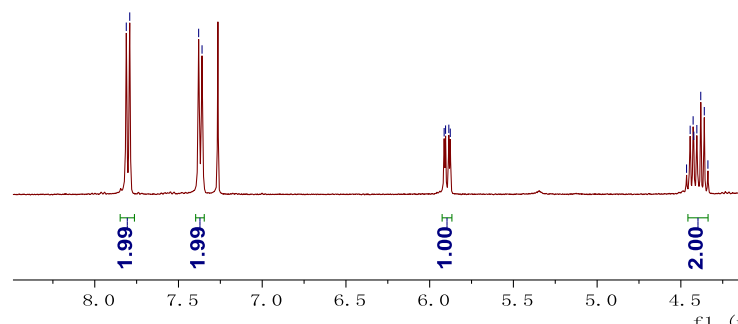

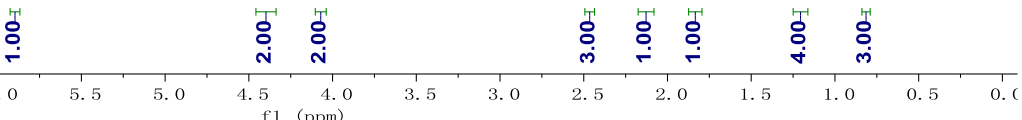

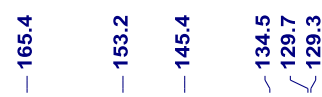

$\overline{0} \frac{9}{8}$

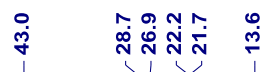

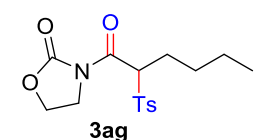

3ag

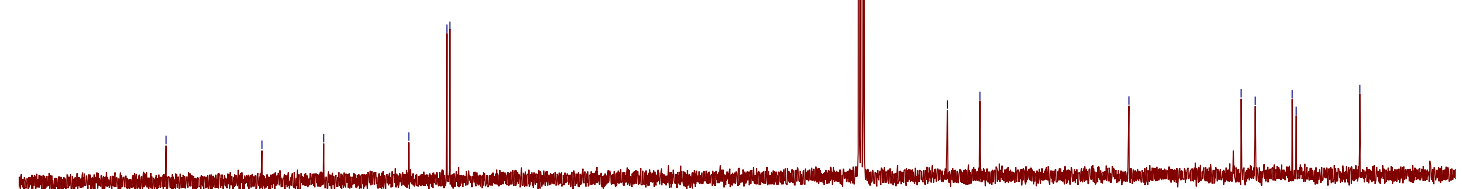

$\begin{array}{llllllllll}180 & 170 & 160 & 150 & 140 & 130 & 120 & 110 & 100 & 90\end{array}$ 
幽 诲

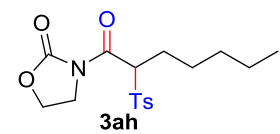

$3 \mathrm{ah}$

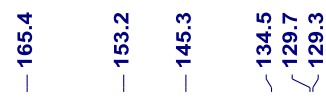

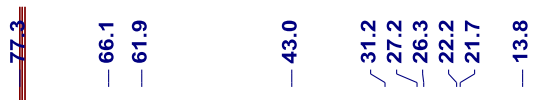
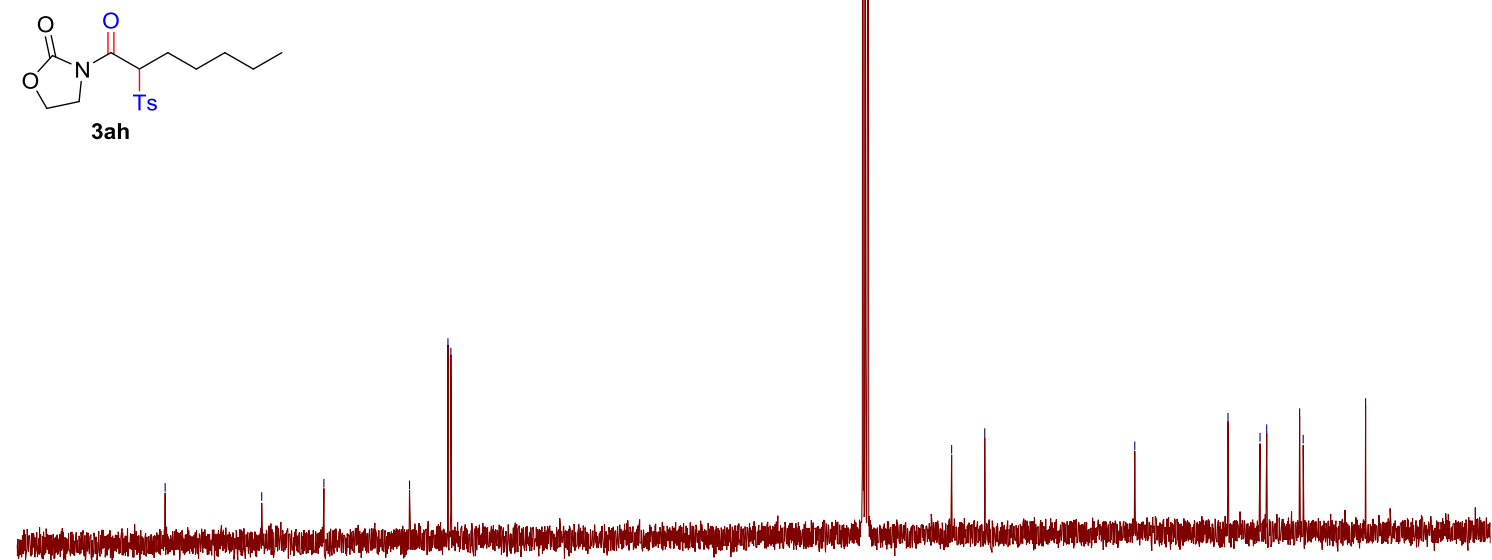

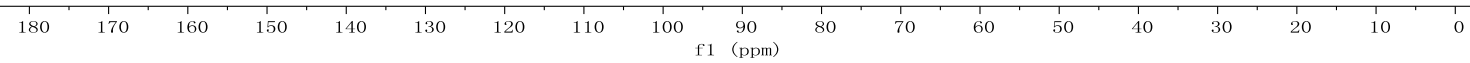




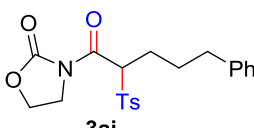

3ai

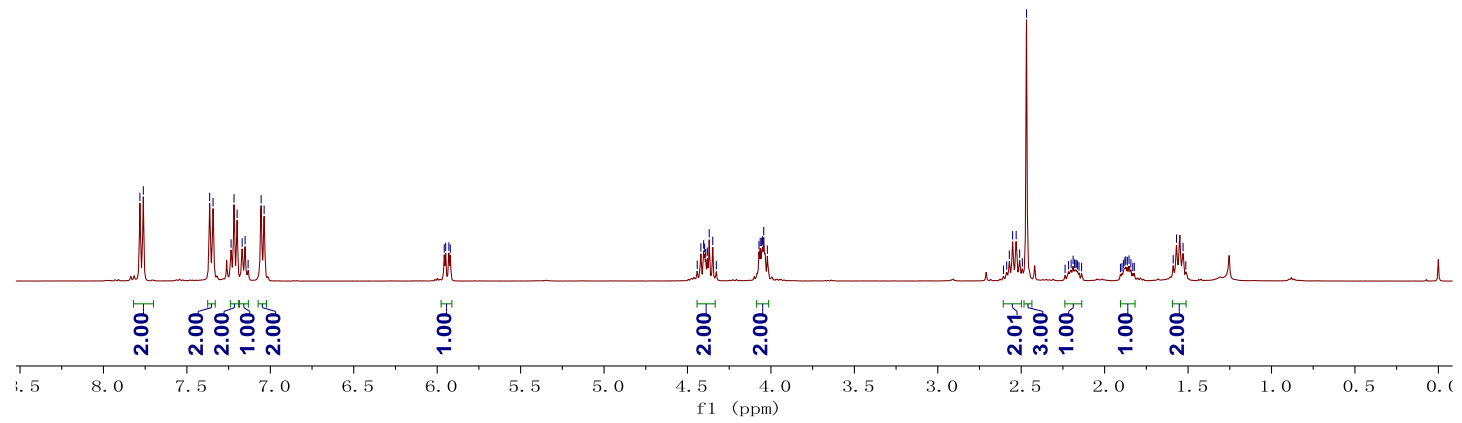

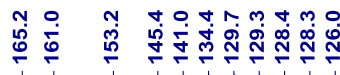

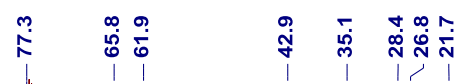

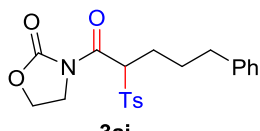

3ai

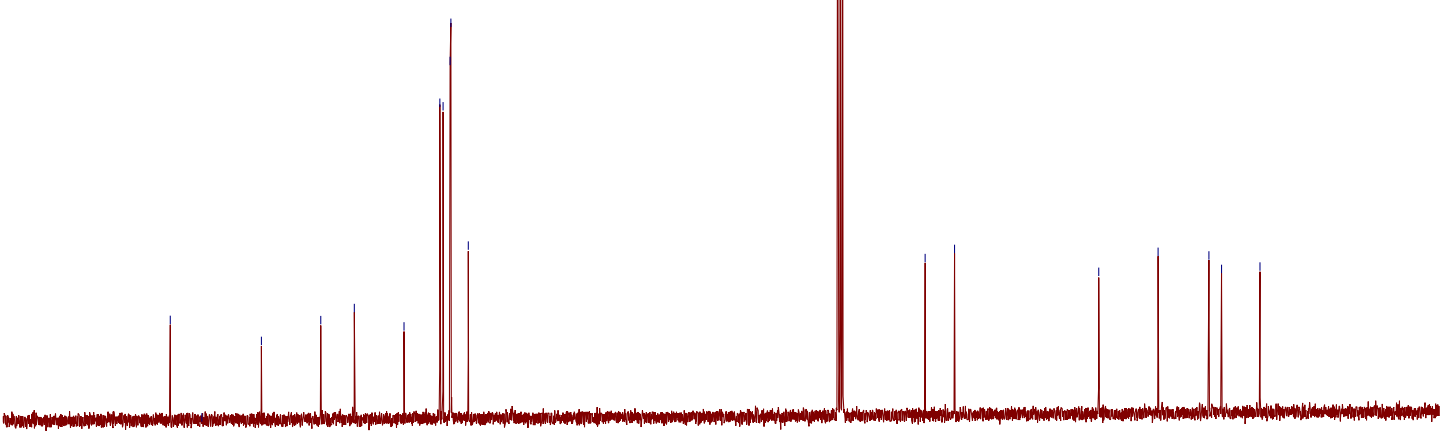

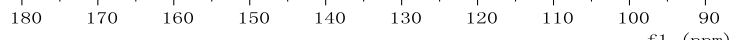

$2010 \quad 0$ 


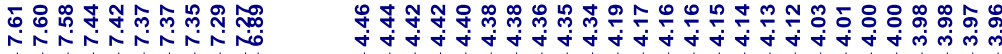

3aj

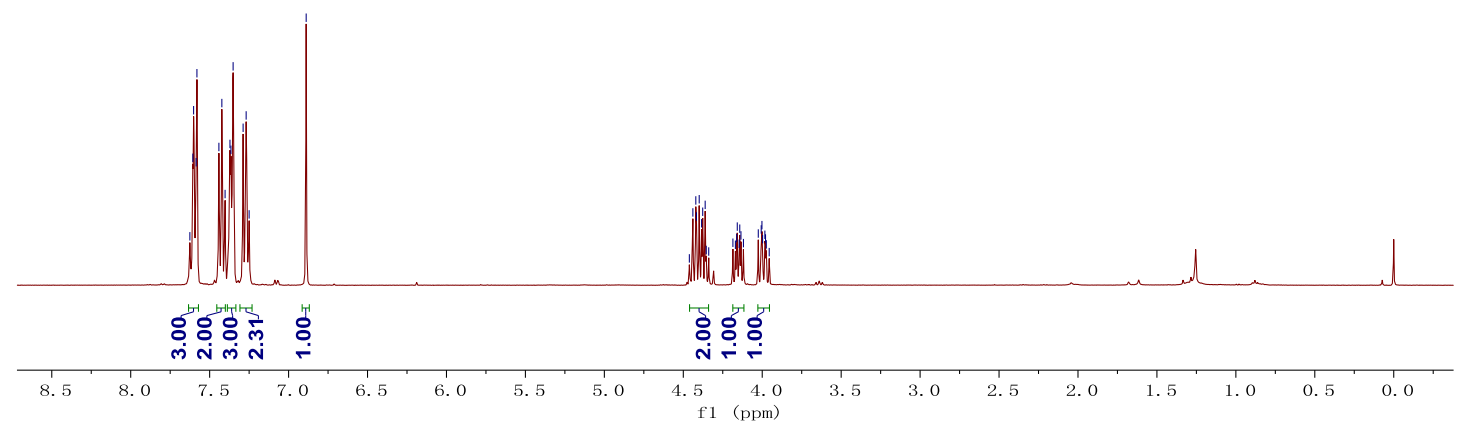

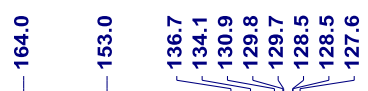

$\stackrel{m}{i} \underset{i}{i} \quad \stackrel{\infty}{i}$
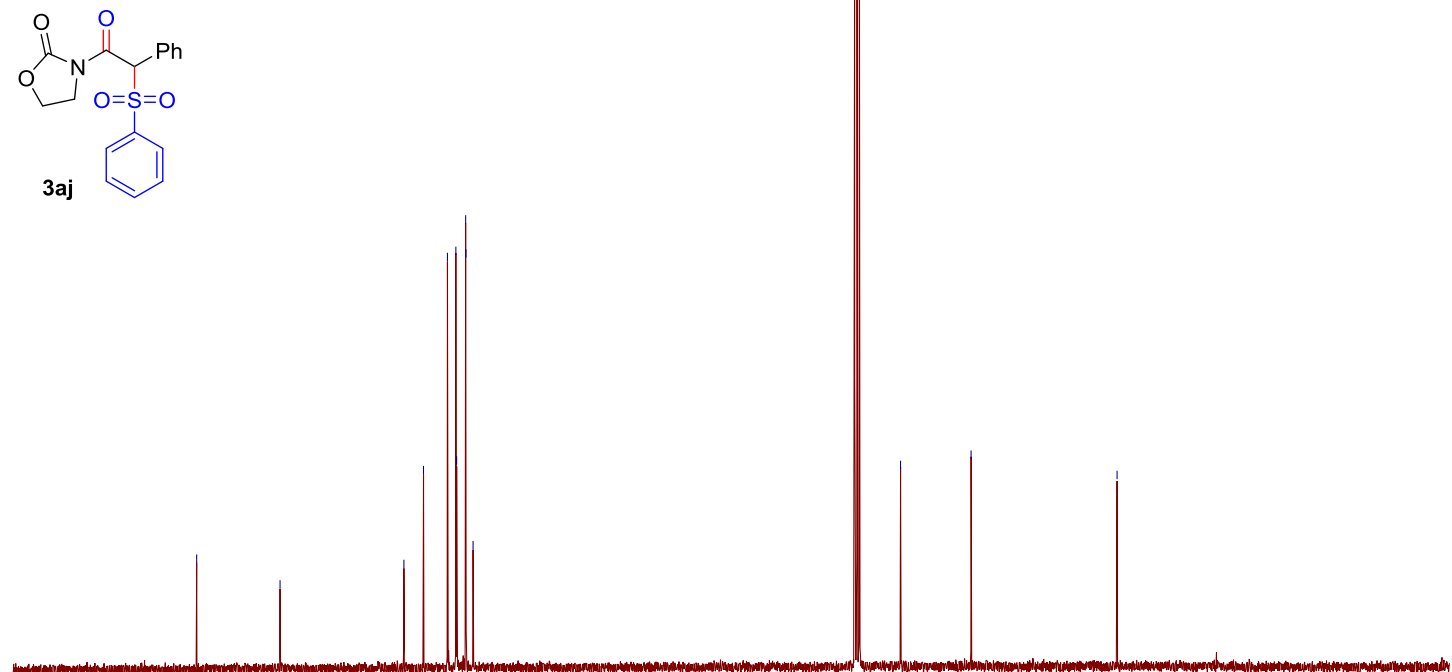

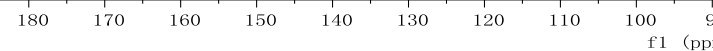



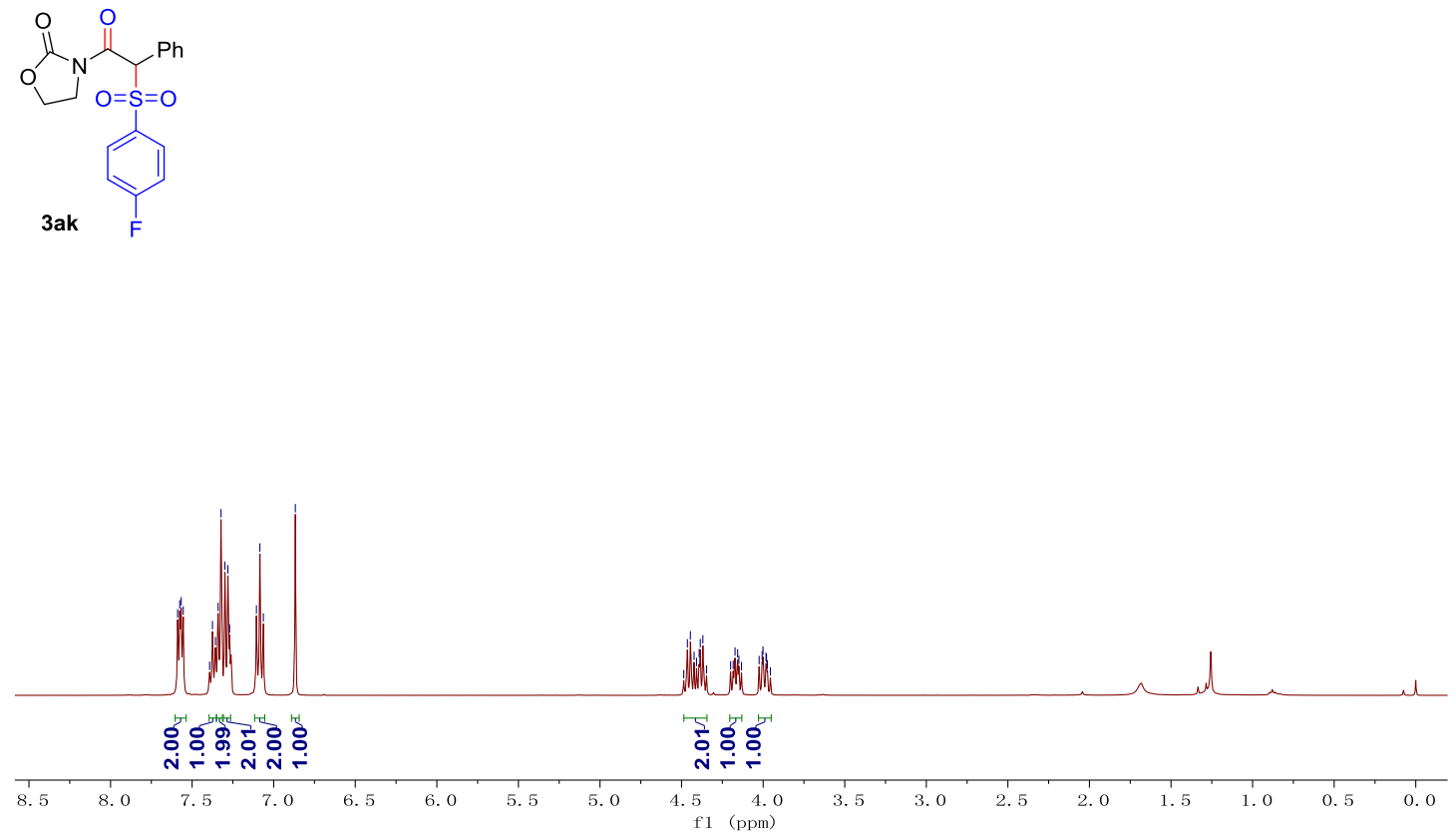

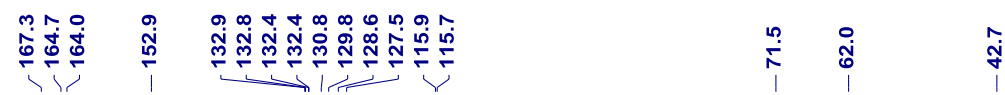
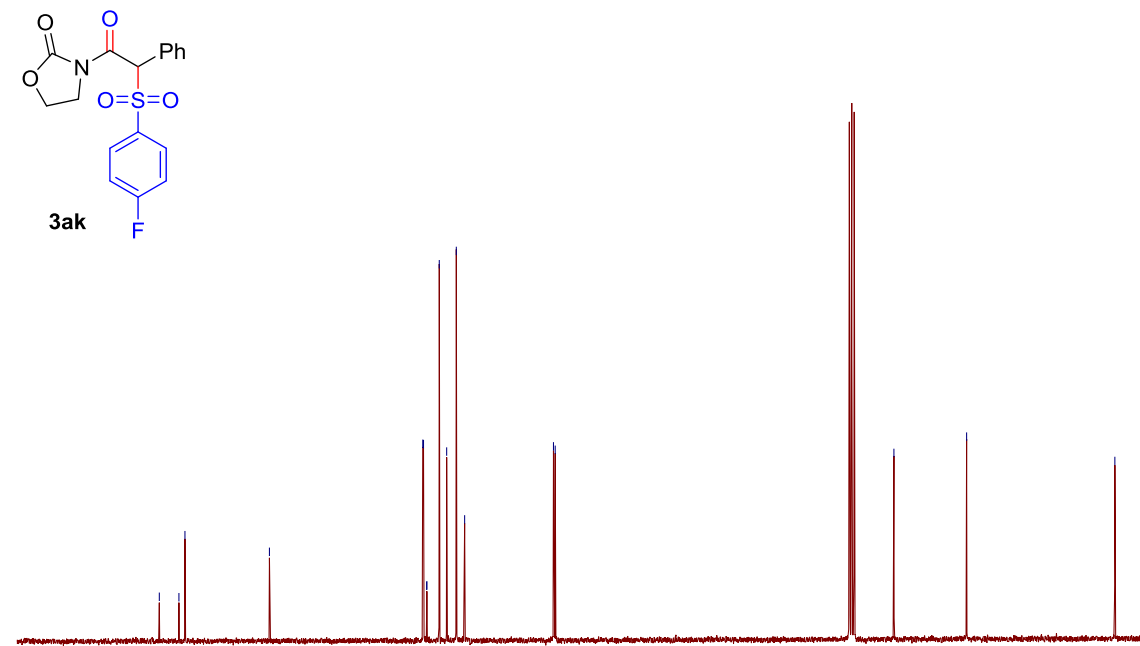
$\begin{array}{llllllllll}180 & 170 & 160 & 150 & 140 & 130 & 120 & 110 & 100 & 90 \\ \mathrm{f} 1 & (\mathrm{ppm})\end{array}$ 

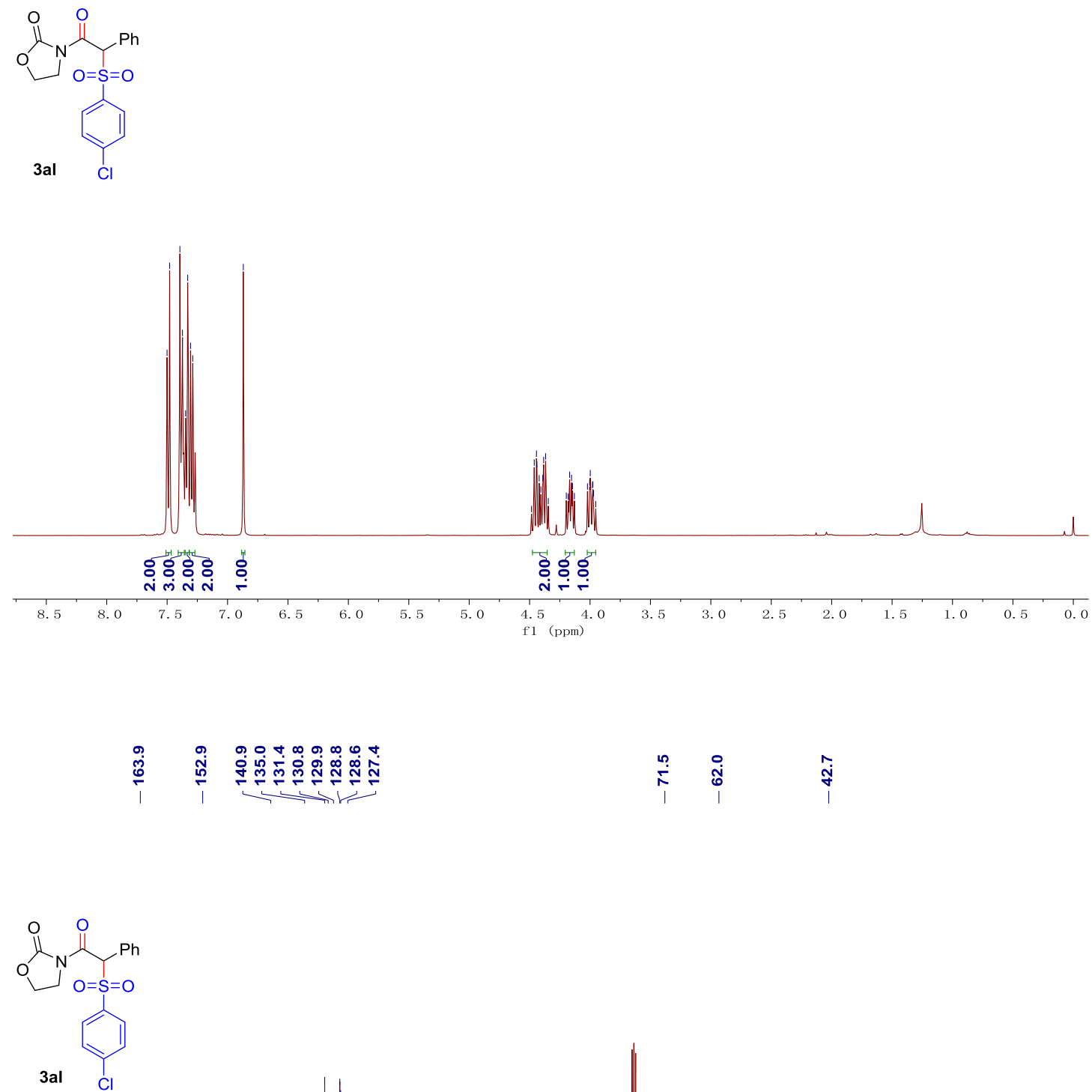

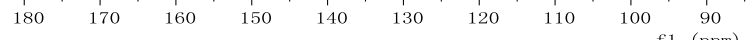

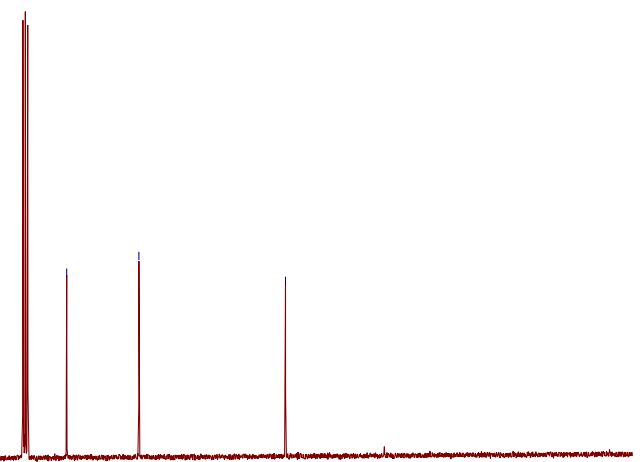




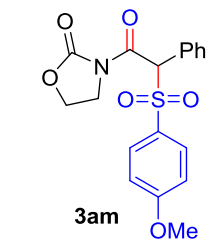

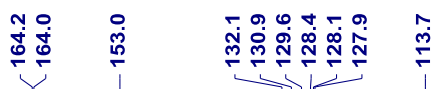

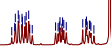

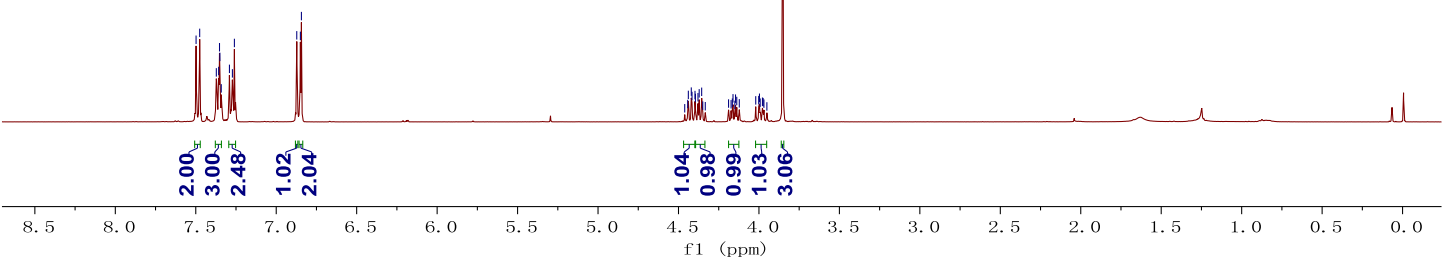

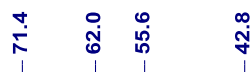
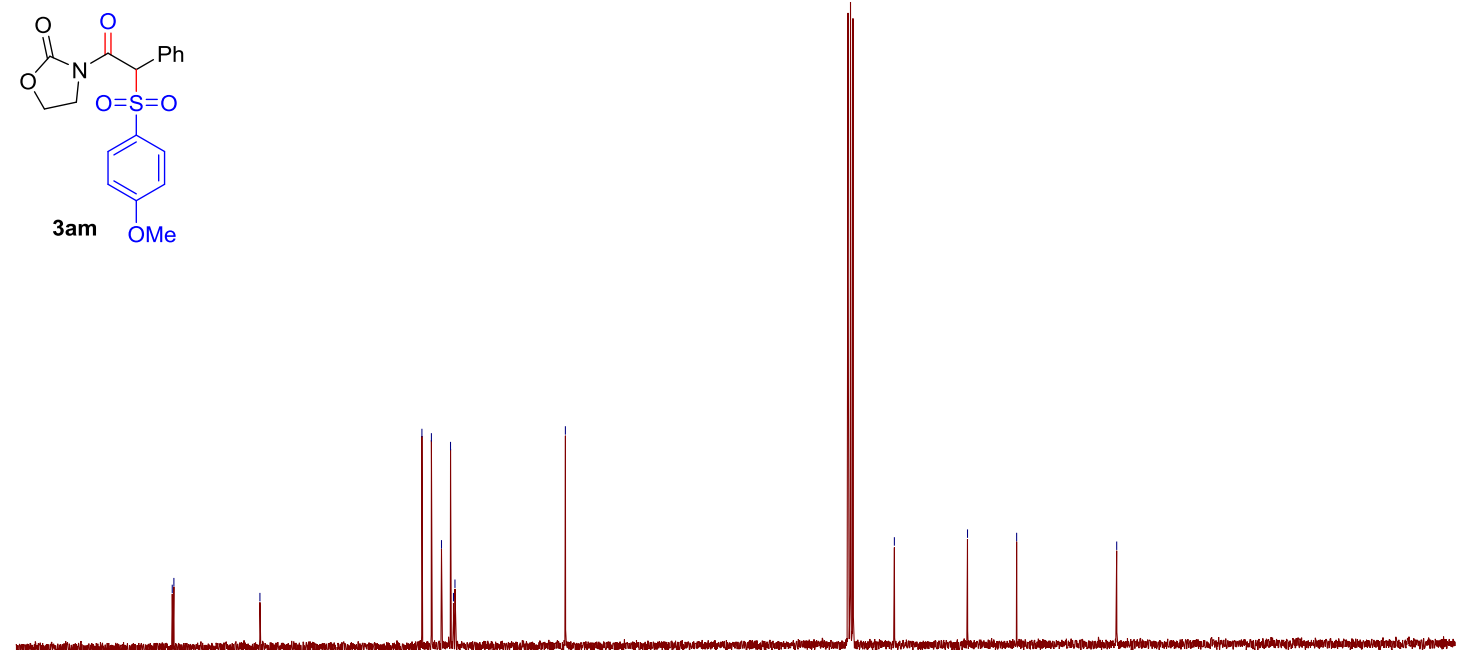

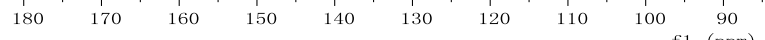



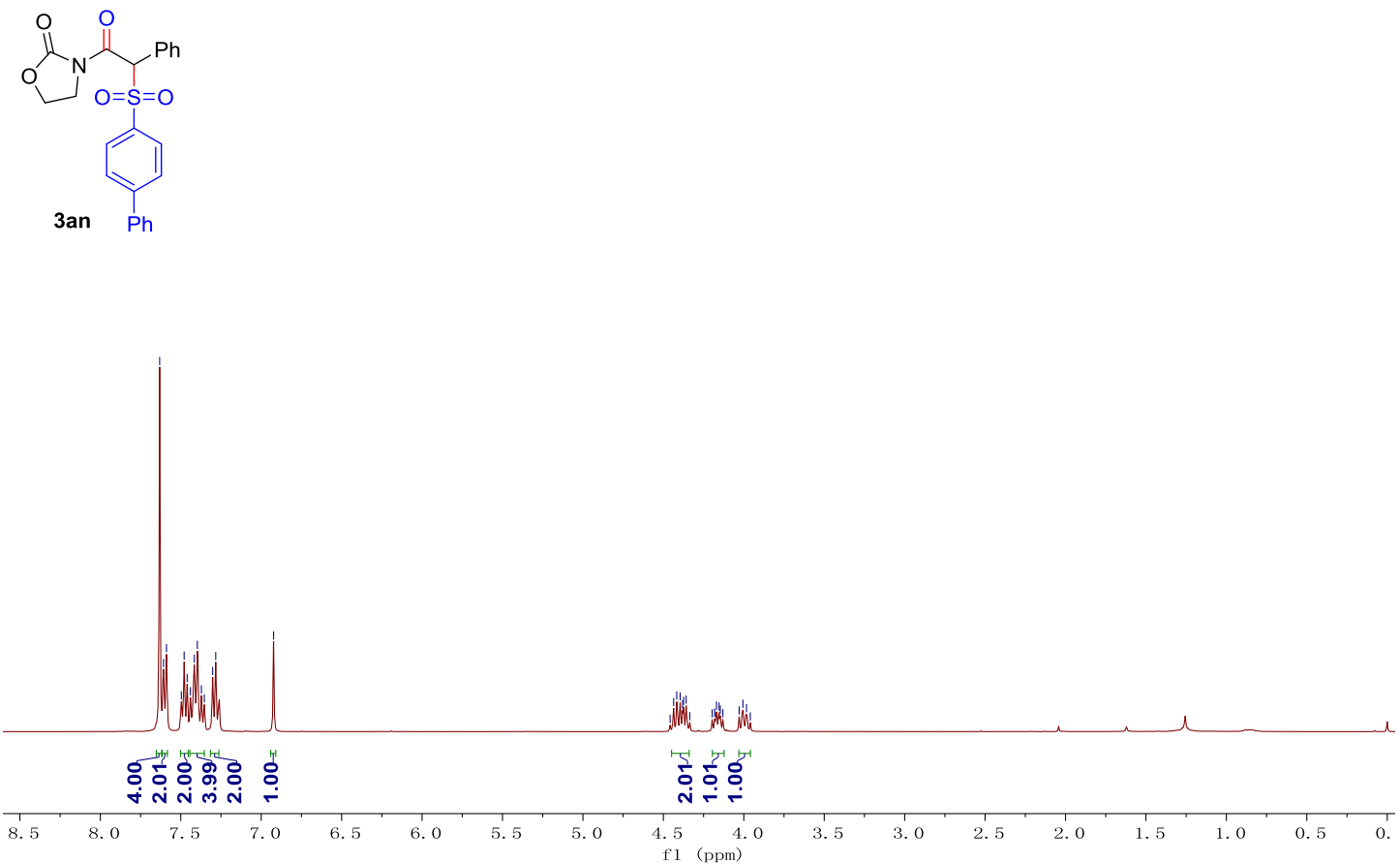

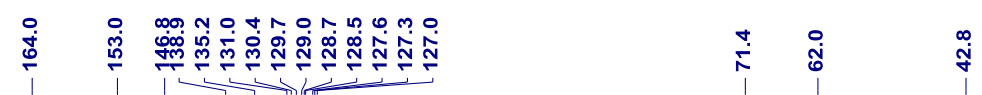
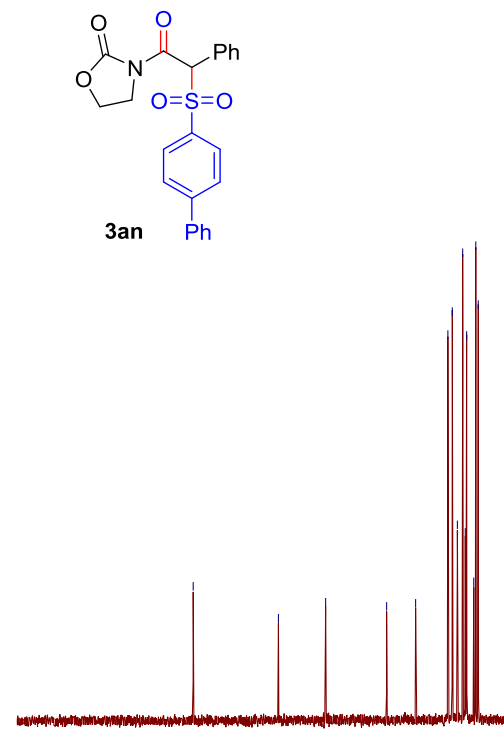
$\begin{array}{rrrrrrrrrr}180 & 170 & 160 & 150 & 140 & 130 & 120 & 110 & 100 & 90 \\ \mathrm{f} 1 & (\mathrm{ppm})\end{array}$ 

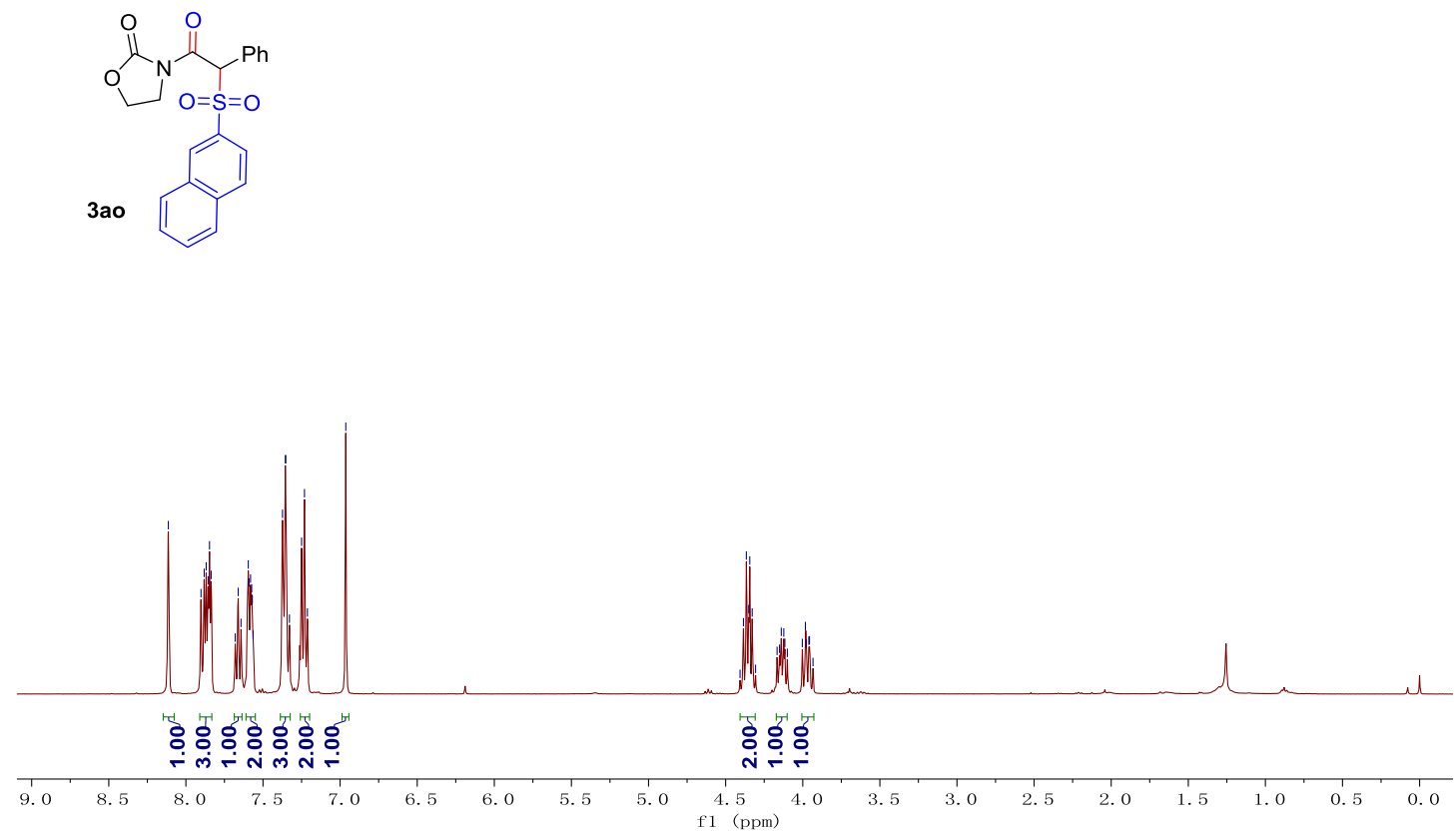

\section{管}

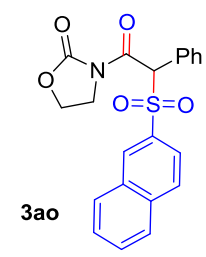

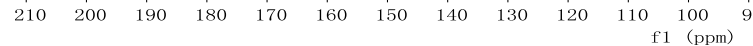



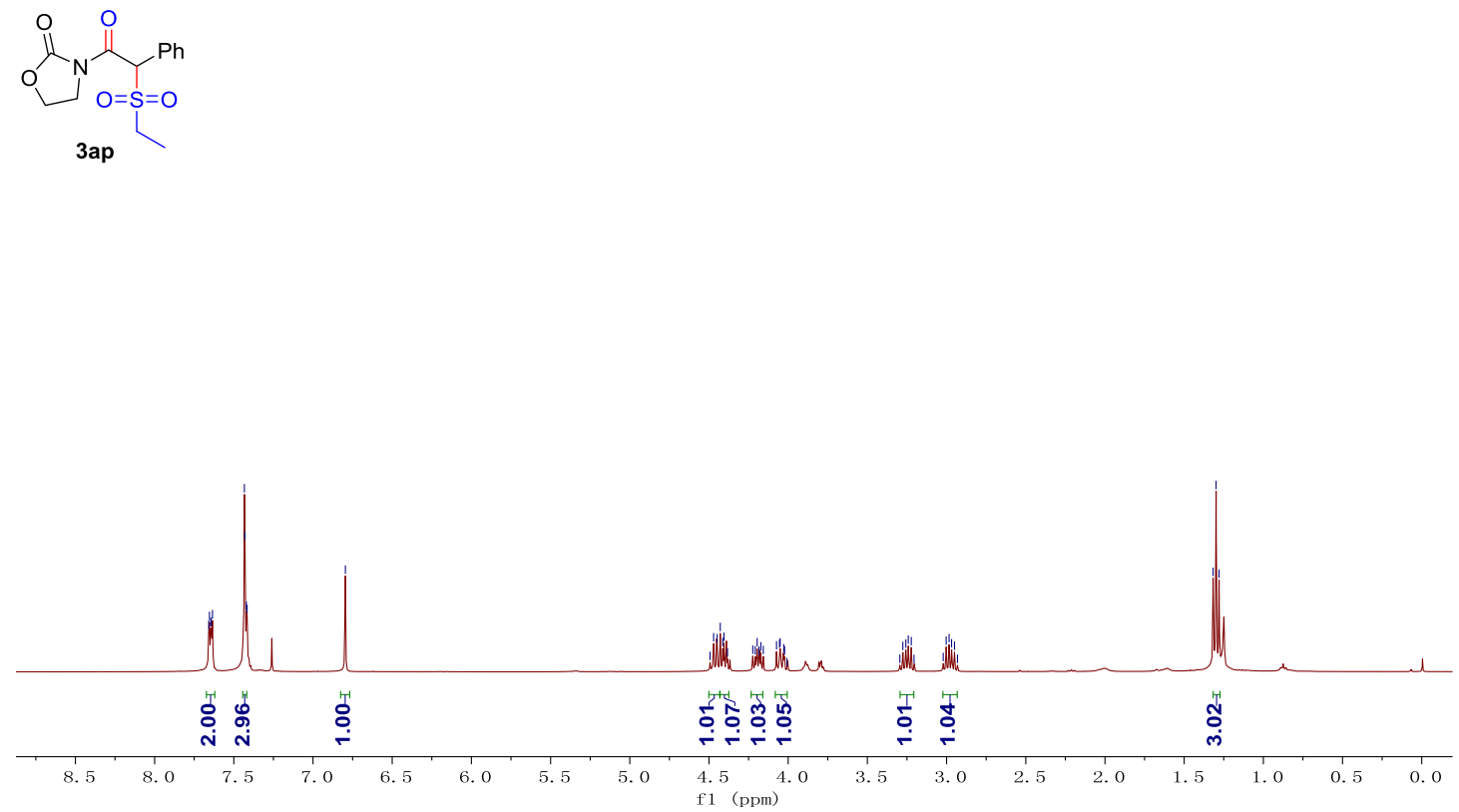

蓆

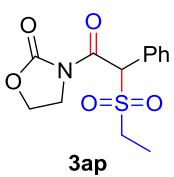

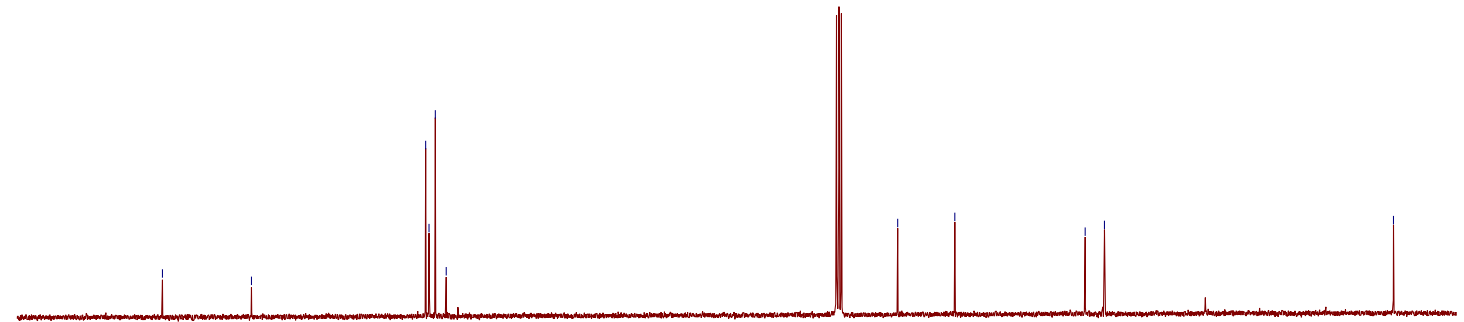

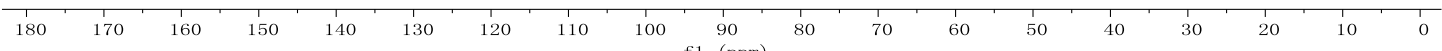




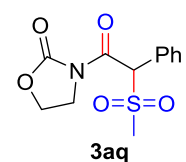

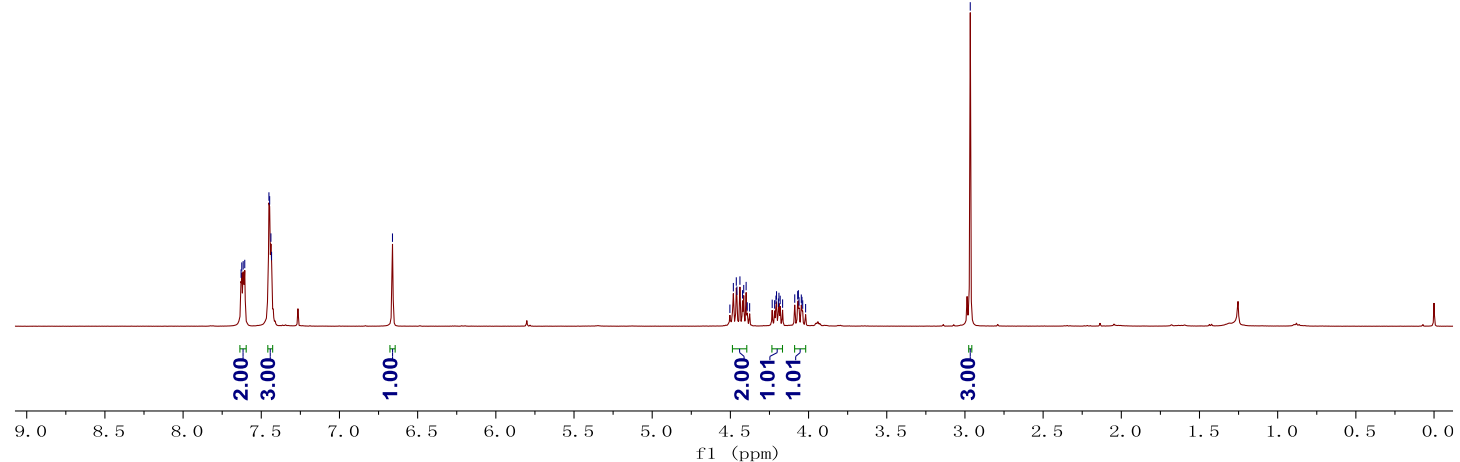

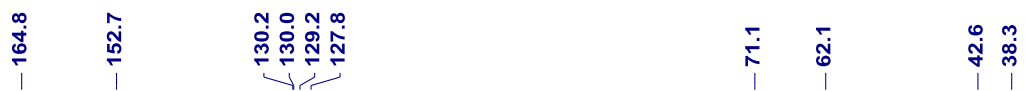

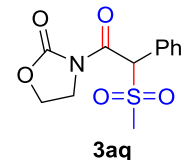

$3 a q$

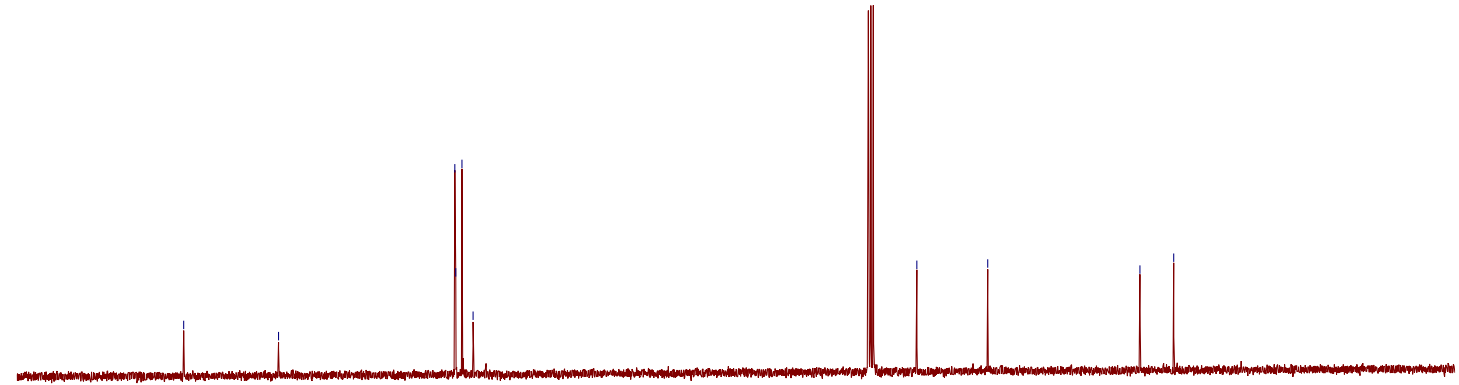

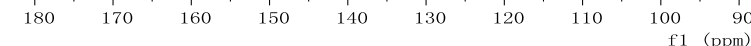



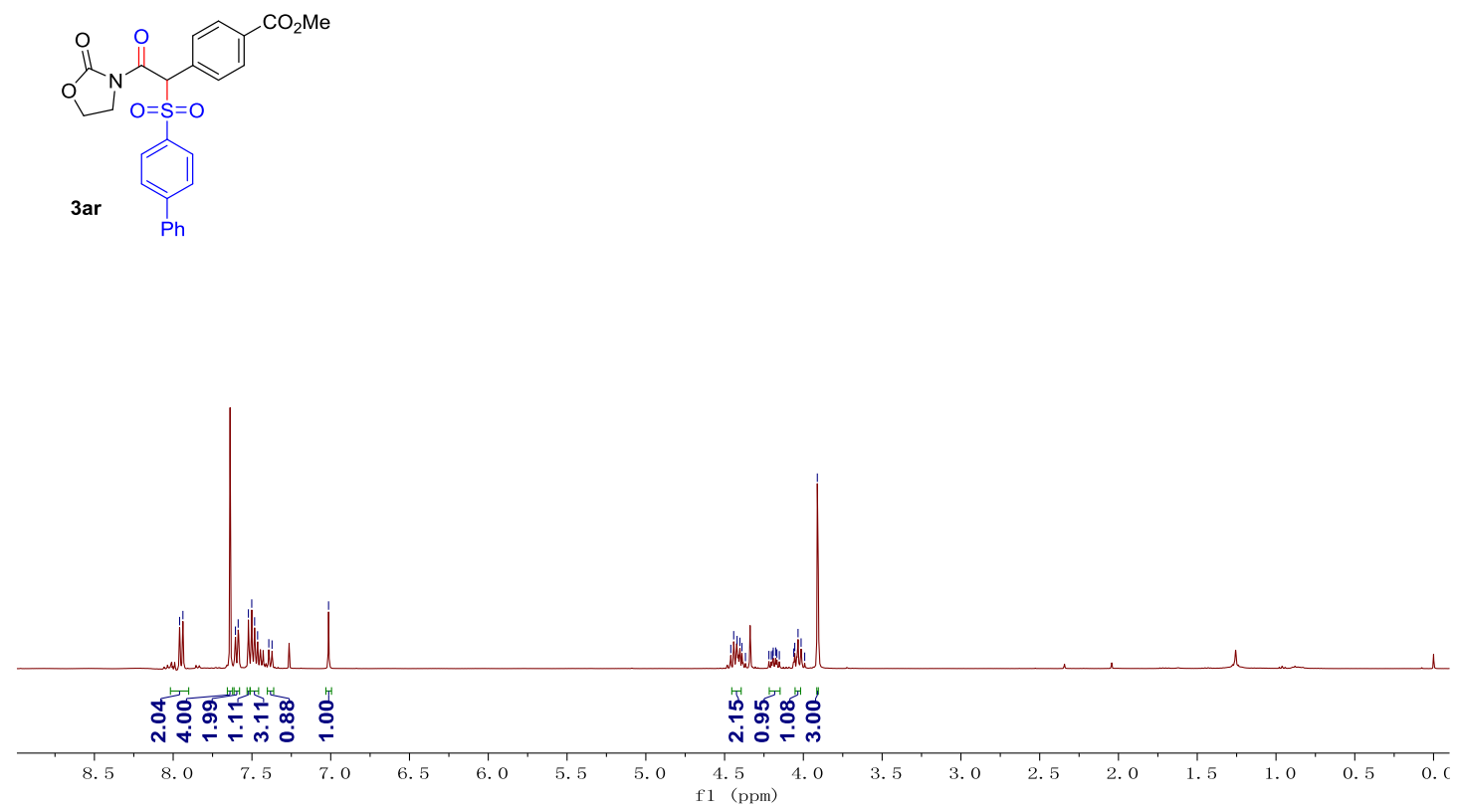

$\underbrace{1}$

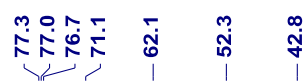
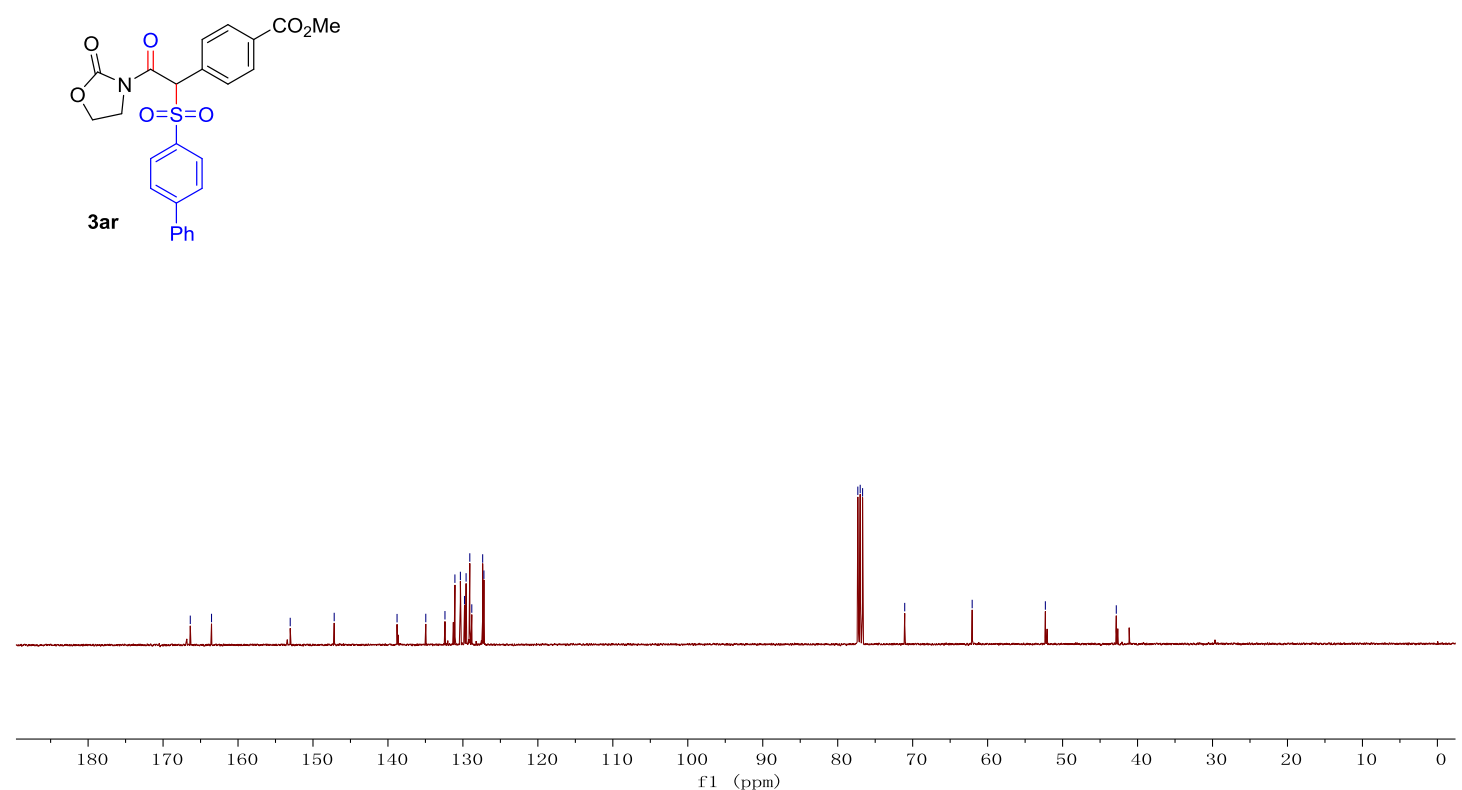


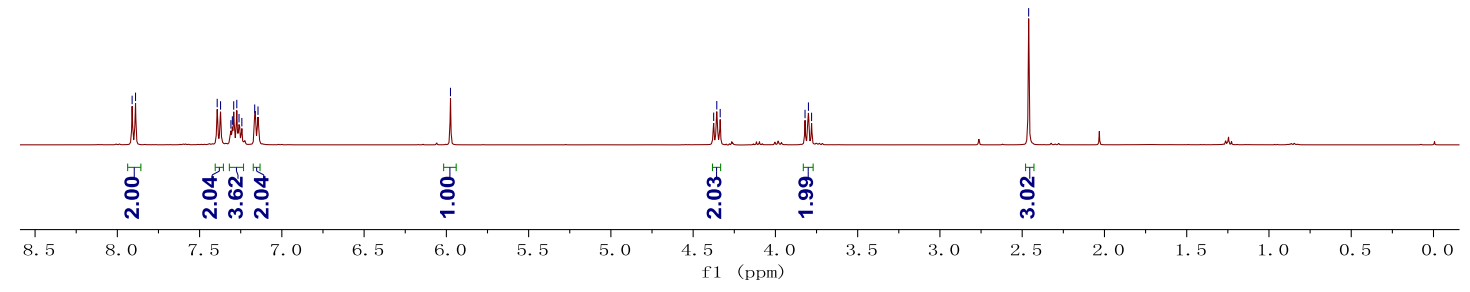

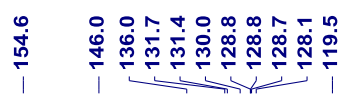

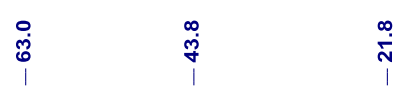

$$
\underbrace{\text { OTs }}_{\text {Ph }} \AA_{Y_{H}^{H}}^{H}
$$

$4 a$
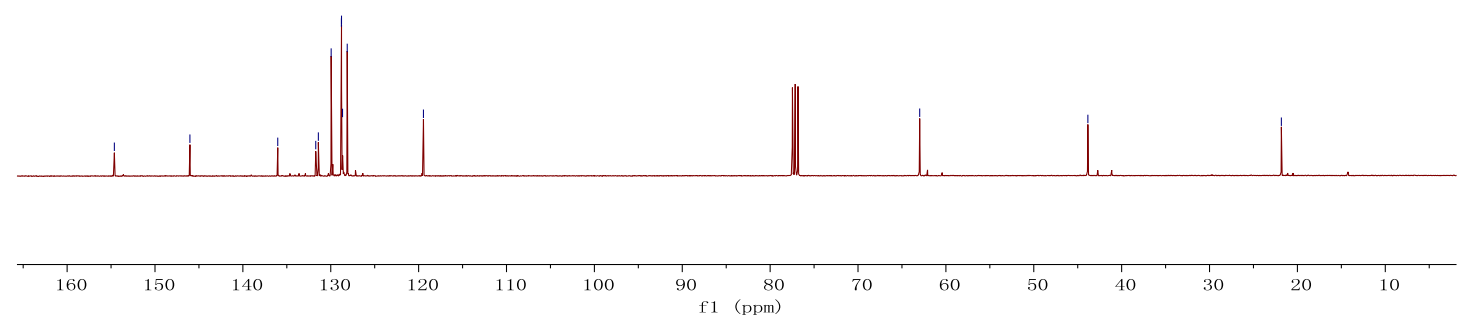


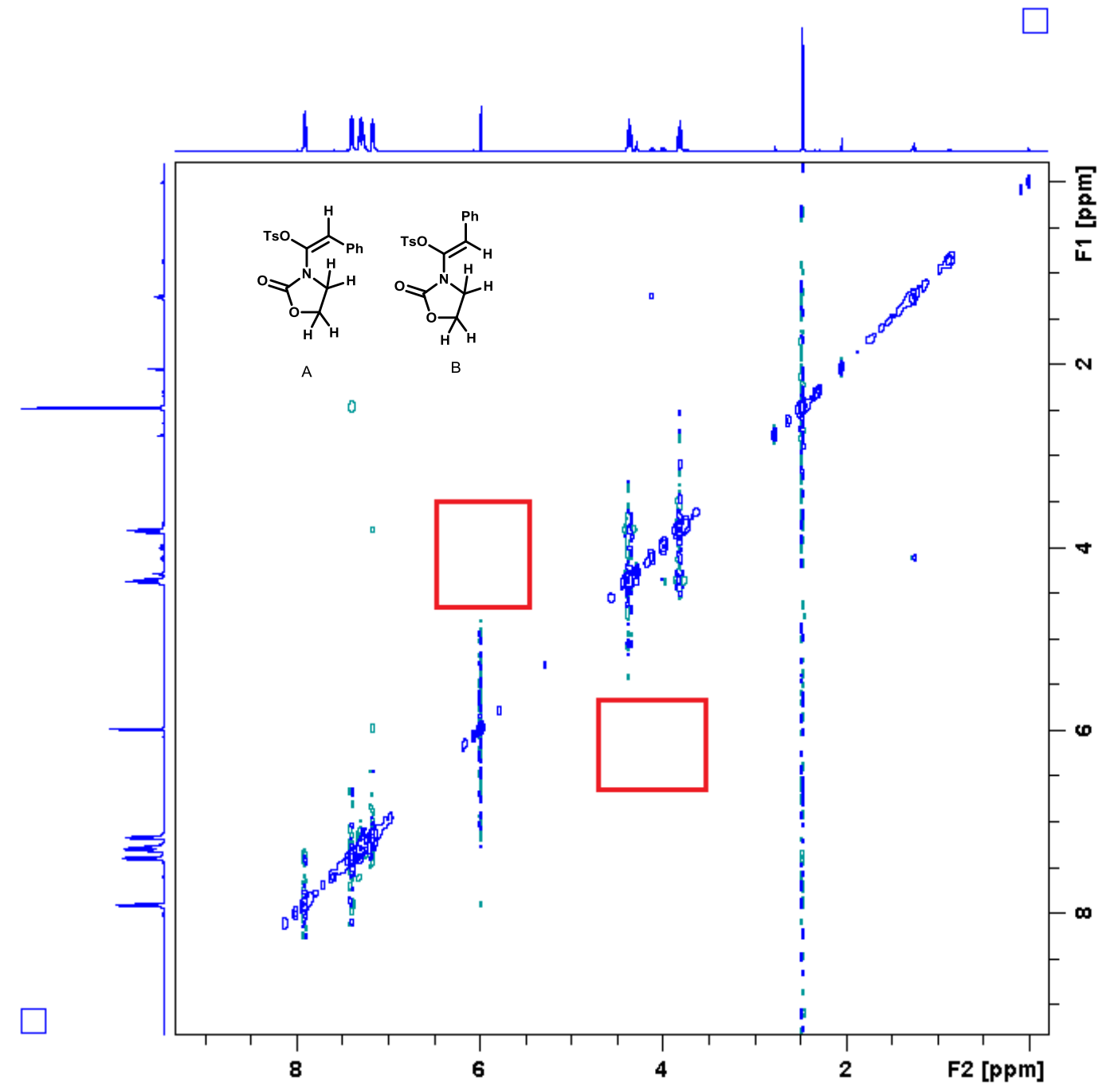

By NOSEY analysis, there is no H-H relevant signal in the red region, so B configuration is excluded. 

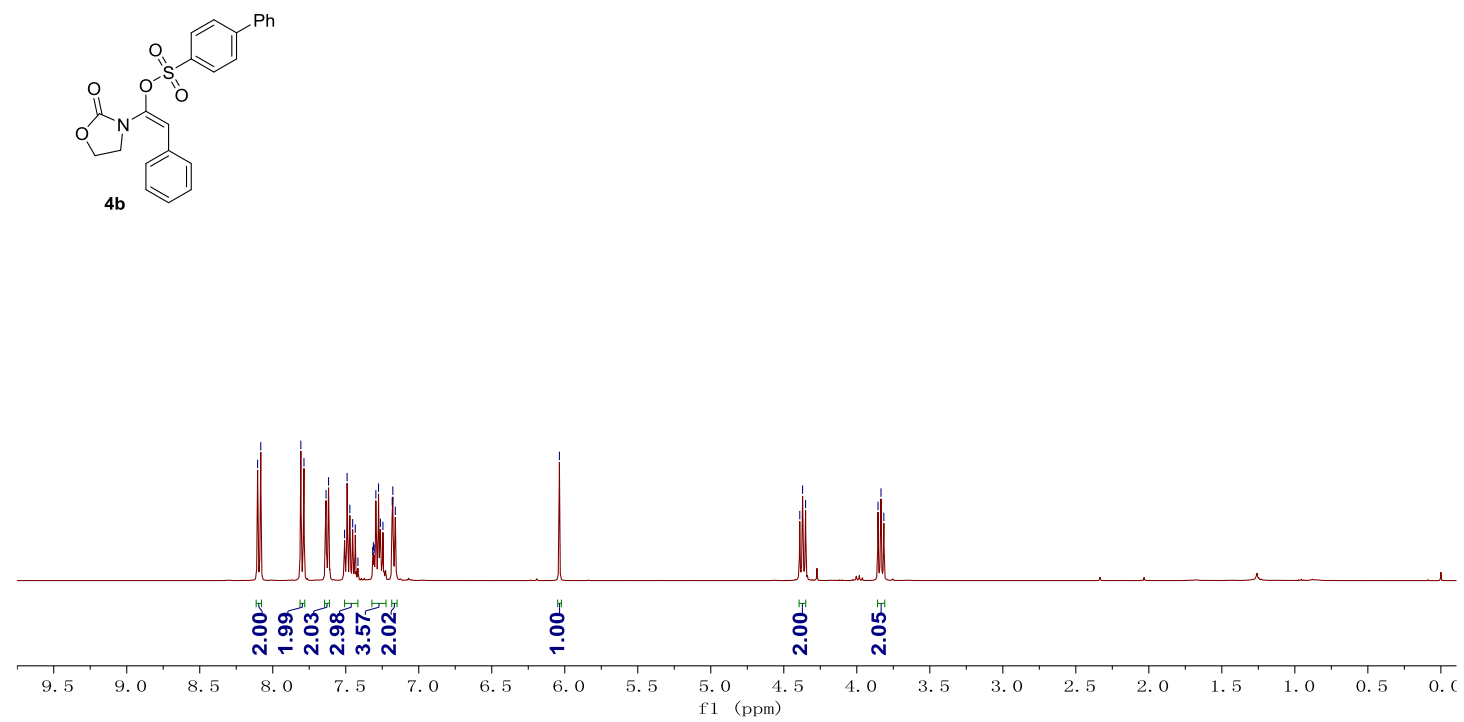

I
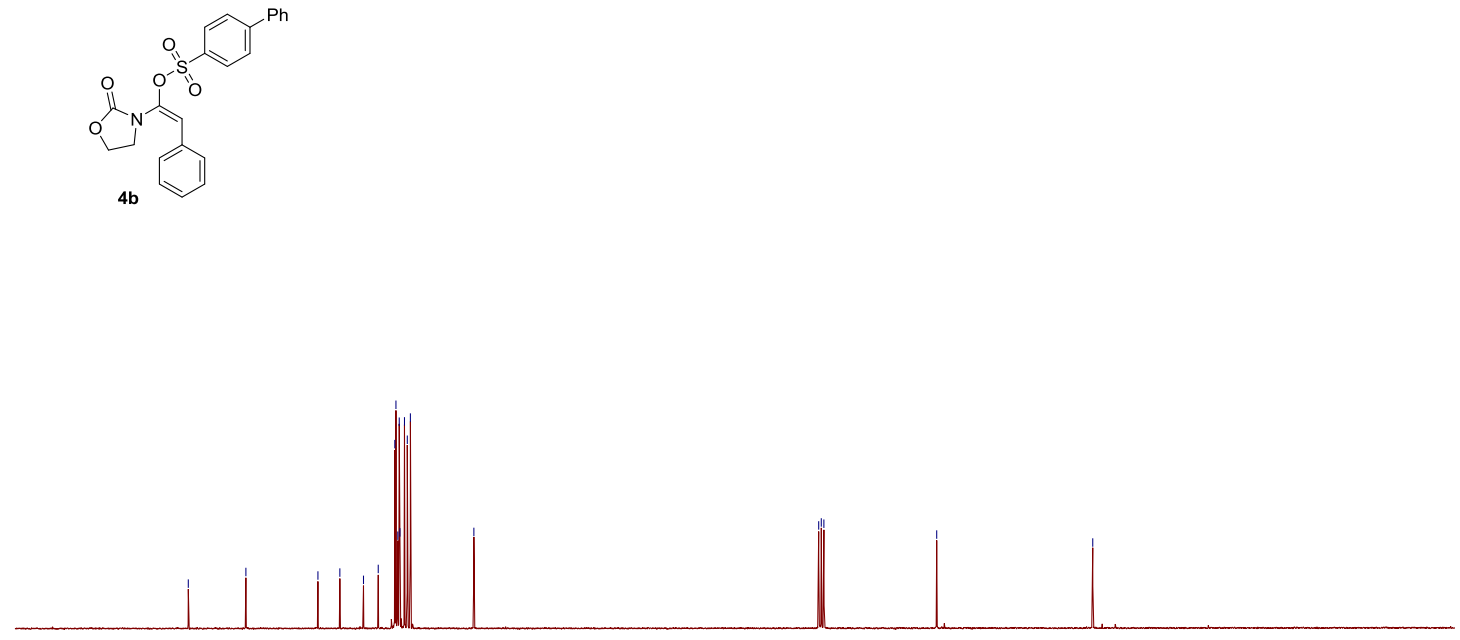

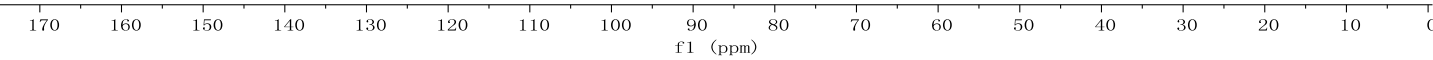



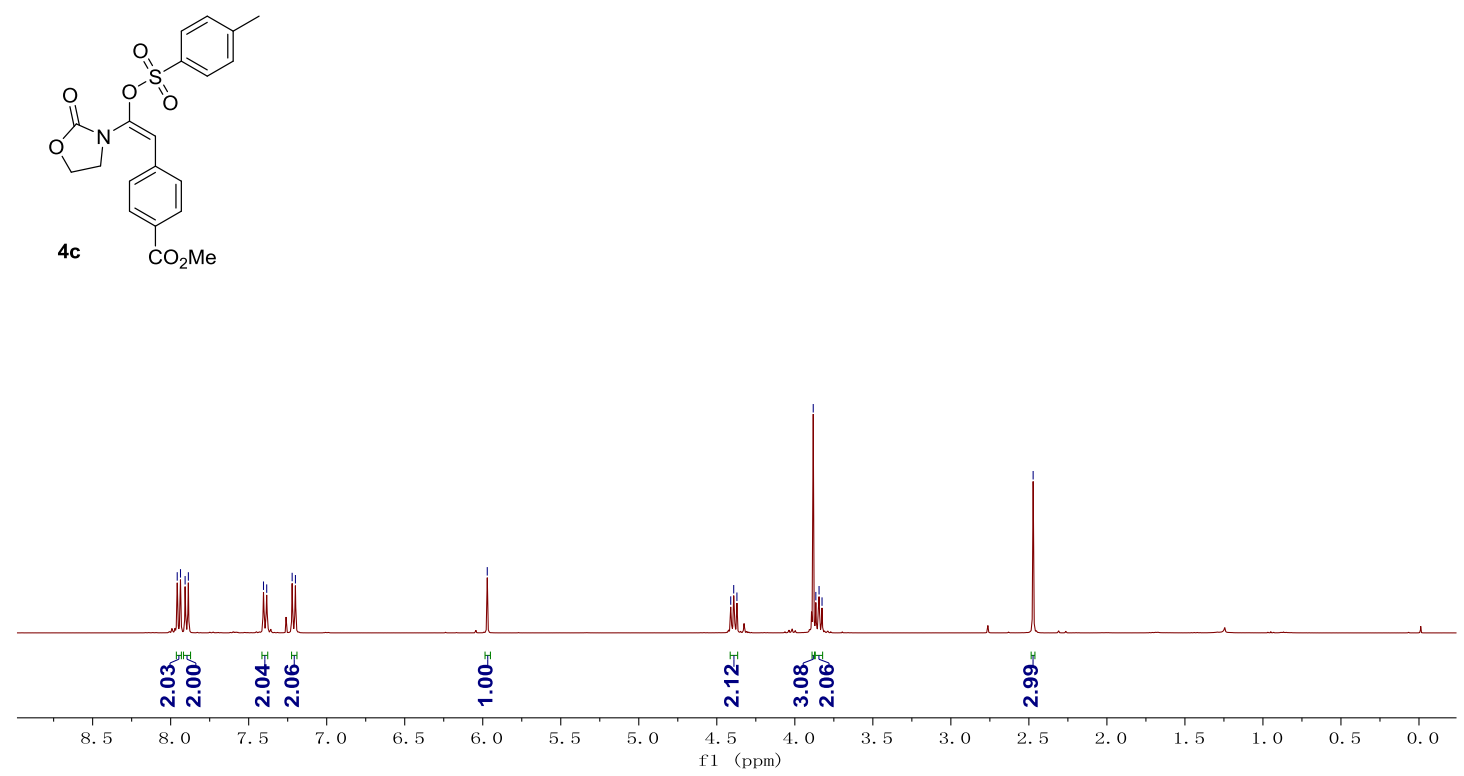

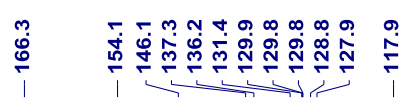

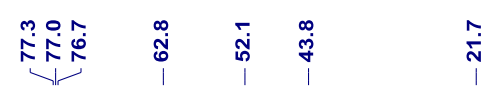
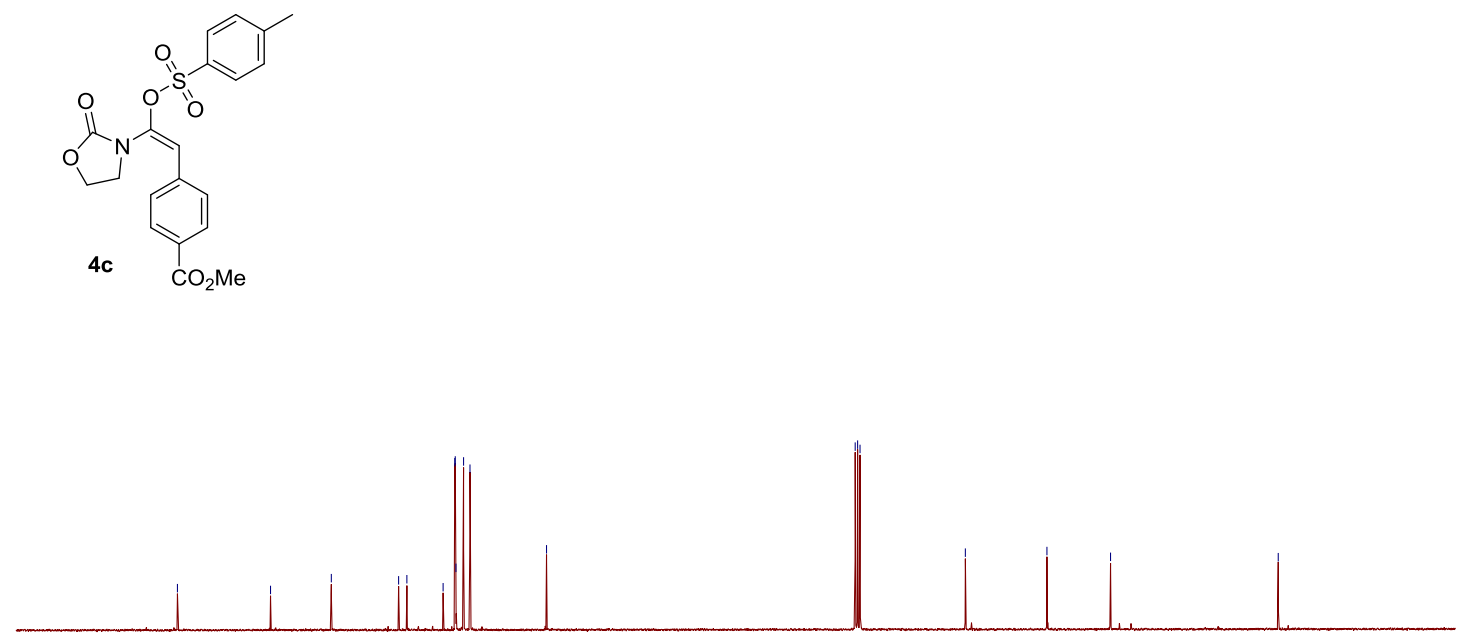

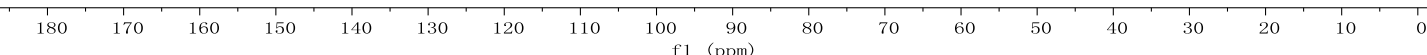

